\author{
Aus der Abteilung Thorax-, Herz- und Gefäßchirurgie \\ (Prof. Dr. med. H. Dalichau) \\ im Zentrum Chirurgie \\ der Medizinischen Fakultät der Universität Göttingen
}

\title{
Einfluß von Dexamethason auf die perioperative Volumenkonstellation koronarchirurgischer Patienten
}

Anwendung optimierter Indikatordilutionsmethoden zur quantitativen Erfassung verschiedener Flüssigkeitskompartimente

\author{
INAUGURAL - DISSERTATION \\ zur Erlangung des Doktorgrades \\ der Medizinischen Fakultät \\ der Georg-August-Universität zu Göttingen
}

\author{
vorgelegt von \\ Savvas Giannaris \\ aus Korinthos/Griechenland
}

Göttingen 1999 
Dekan:

I. Berichterstatter:

II. Berichterstatter/in:

III. Berichterstatter/in:

Tag der mündlichen Prüfung:
Prof. Dr. med. M. Droese

Priv.-Doz. Dr. med. B. Schorn 


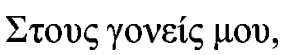

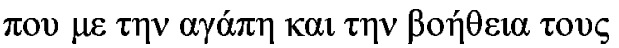

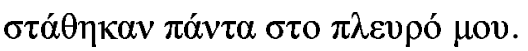




\section{Abkürzungen}

$E_{\lambda}^{l w}$

$\bar{E}_{\lambda}$

$1 h$

$6 h$

$20 h$

APd

APs

ARDS

AUC

CI

$C P B$

DXM

EKZ

EVGW

EVLW

$\mathrm{Hb}$

$H f$

$H k t$

HLM

HZV

ICG

$I L$

KOF

LPS

MAP

MPAP

$M W, \bar{x}$

n.N.

$O p A$

$O p E$

PAPd

PAPS

PCWP

PLC

PVRI
Plasmaeigenextinktion einer Plasmaprobe bei $\lambda \mathrm{nm}$ (Leerwertprobe)

korrigierte Extinktion einer ICG-haltigen Plasmaprobe bei $\lambda \mathrm{nm}$

Meßzeitpunkt „eine Stunde nach Operationsende“

Meßzeitpunkt „sechs Stunden nach Operationsende“

Meßzeitpunkt „zwanzig Stunden nach Operationsende“

Diastolischer arterieller Druck

Systolischer arterieller Druck

Adult respiratory distress syndrome

Fläche unter einer Indikatordilutionskurve (,area under curve“)

Herzzeitvolumenindex (,cardiac index“)

Kardiopulmonaler Bypass unter Einsatz der Herz-Lungen-Maschine

Dexamethason-Gruppe der Studienpatienten

Extrakorporale Zirkulation

„Extravaskuläres Gesamtwasser“

„Extravaskuläres Lungenwasser“

Hämoglobin-Konzentration

Herzfrequenz

Hämatokrit

Herz-Lungen-Maschine

Herzzeitvolumen

Indocyaningrün

Interleukin

Körperoberfläche

Lipopolysaccharide

Arterieller Mitteldruck

Pulmonalarterieller Mitteldruck

Arithmetisches Mittel

Meßpunkt ,nach Einleitung der Narkose“

Operationsanfang, entspricht dem n.N. Meßzeitpunkt

Operationsende

Diastolischer pulmonalarterieller Druck

Systolischer pulmonalarterieller Druck

Pulmonalkapillärer Verschlußdruck

Placebo-Gruppe der Studienpatienten (Kontrollgruppe)

Pulmonaler Gefäßwiderstandsindex 


$\begin{array}{ll}s d & \text { Standardabweichung des Mittelwertes } \\ \text { sem } & \text { Standardfehler des Mittelwertes } \\ S V I & \text { Schlagvolumenindex } \\ S V R I & \text { Peripherer Gefäßwiderstandsindex } \\ T P & \text { Gesamteiweiß-Konzentration } \\ v . N . & \text { Meßpunkt „vor Einleitung der Narkose“ } \\ V_{d-\text { ges }} & \text { "Gesamtes“ Blutvolumen } \\ V_{d-p l a s m a} & \text { "Gesamtes“ Plasmavolumen } \\ V_{d-z e n t} & \text { „Zentrales“ Blutvolumen } \\ V_{d-\text {-zirk }} & \text { „Zirkulierendes“ Blutvolumen } \\ Z T V & \text { „Zentrales thermoakzessibles“ Volumen } \\ Z V D & \text { Zentraler Venendruck }\end{array}$




\section{Inhaltsverzeichnis}

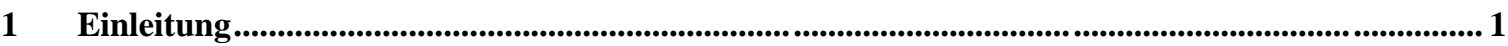

2 Theoretische Grundlagen der Indikatordilution ....................................................................................... 3

2.1 Indikatoren und Auswertungsprinzipien in der Dilutionstechnik .................................................. 3

2.1.1 Das Prinzip der Massenerhaltung.......................................................................................... 4

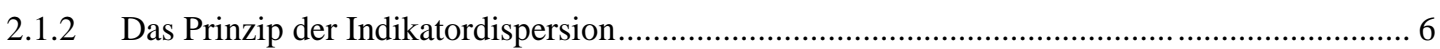

2.1.3 Die stochastische Systemanalyse .......................................................................... 7

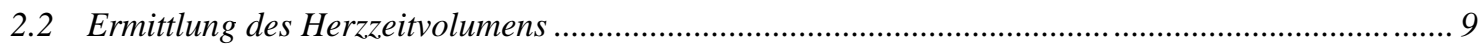

2.3 „Zentrales thermoakzessibles “ Volumen................................................................................. 11

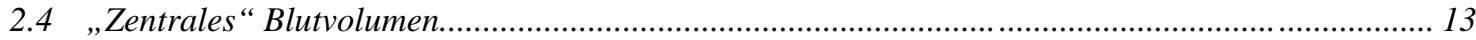

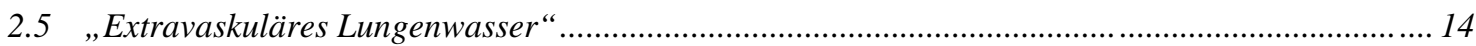

2.6 „In vivo“-Bestimmung des ,zirkulierenden“ Blutvolumens .......................................................... 15

2.6.1 Die Kreislauf-Transportfunktion und der rekursive Faltungsalgorithmus ............................ 15

2.6.2 Das zweikompartimentelle Modell für die Kreislauf-Transportfunktion .............................. 17

2.6.3 Verteilungsvolumina auf der Basis des mathematischen Rezirkulationsmodells .................... 18

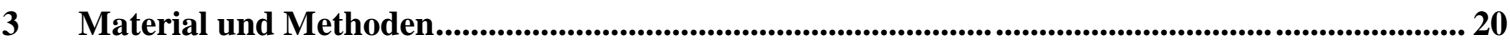

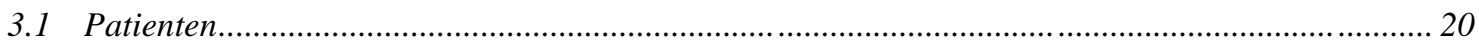

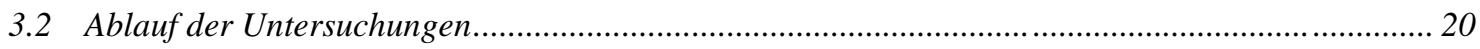

3.2.1 Kathetherisierung der Patienten und Narkoseeinleitung .................................................... 21

3.2.2 Operationsablauf unter Einsatz der extrakorporalen Zirkulation ......................................... 22

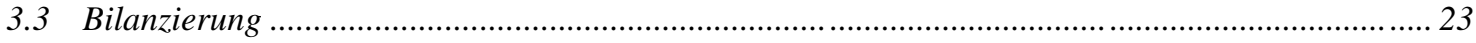

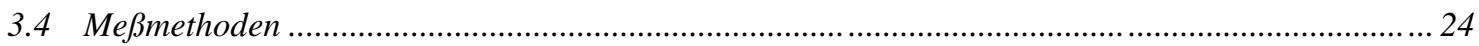

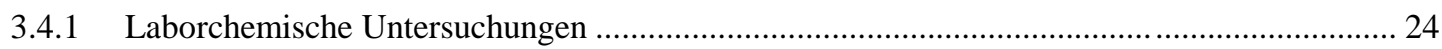

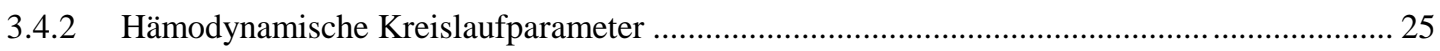

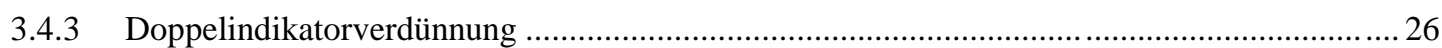

3.4.4 „In vitro“-Bestimmung des ,gesamten“ Blutvolumens ..................................................... 29

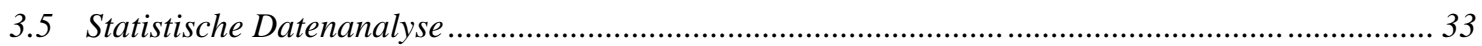

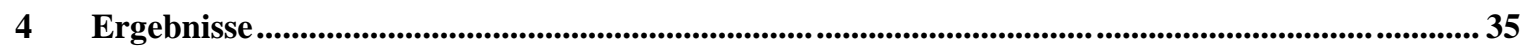

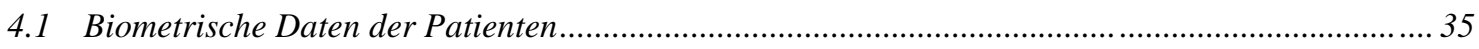

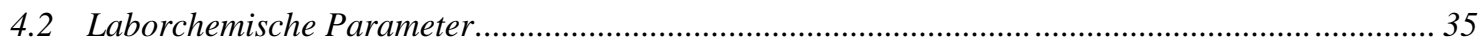

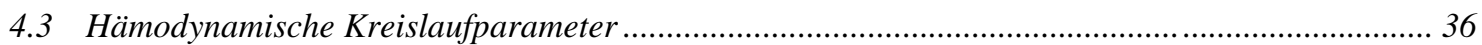

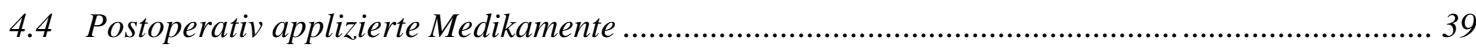


4.5 Flüssigkeitsverteilung im intrathorakalen Raum................................................................... 40

4.6 „Zirkulierendes “ und ,gesamtes “ Blutvolumen .............................................................................. 41

4.7 Verteilung vom Flu $\beta$ und Volumen im großen Kreislauf................................................................ 43

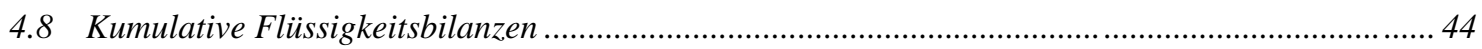

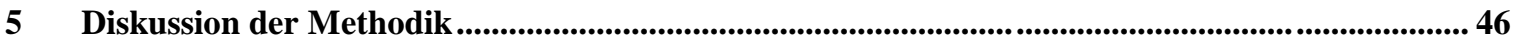

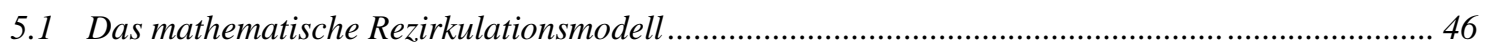

5.2 Kompartimentelle Analyse der Farbstoffdilutionskurven ............................................................ 48

5.3 Ermittlung des „extravaskulären Lungenwassers “ ................................................................ 49

5.4 Optimierte Algorithmen zur Bestimmung des ,, gesamten “ Blutvolumens ........................................ 50

5.4.1 Korrektur der Extinktionsspektren gegenüber dem Leerwertspektrum ...................................51

5.4.2 Kompartimentelle Analyse der Indikatorelimination ........................................................ 53

5.4.3 Rückextrapolation auf dem Zeitpunkt der ersten Leberpassage ..............................................56

5.4.4 Validierung der erhobenen Daten für das ,gesamte“ Blutvolumen.........................................57

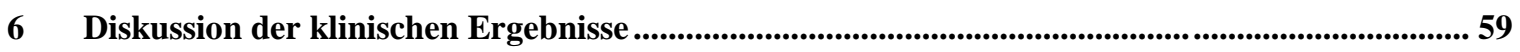

6.1 Kausale Pathogenese des , systemic inflammatory response syndrome “......................................59

6.1.1 Ätiologisch wirksame inflammatorische Faktoren ..........................................................59

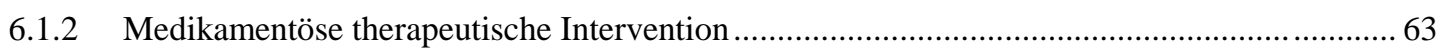

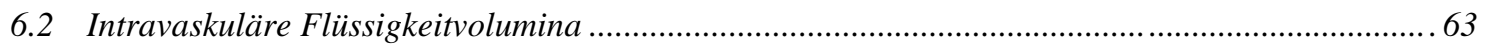

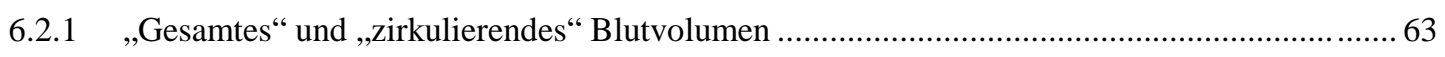

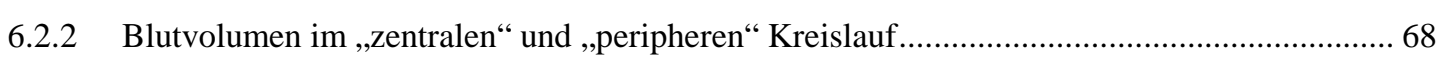

6.2.3 Flüssigkeitskompartimente des großen Kreislaufs ..................................................... 71

6.3 Flüssigkeitsbilanzen und Volumenaustritt in den extravaskulären Raum .................................... 73

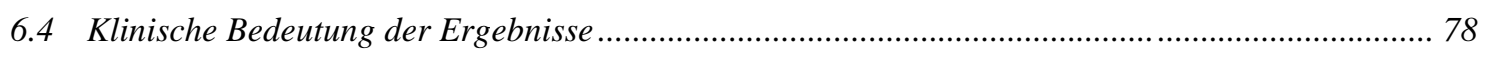

$7 \quad$ Zusammenfassung ........................................................................................................................................ 80

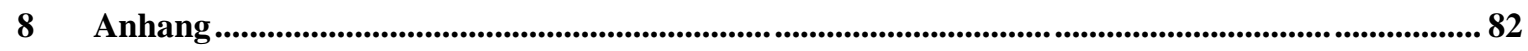

8.1 Parameter der berechneten Kreislauf-Transportfunktionen...................................................... 82

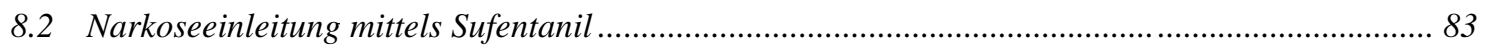

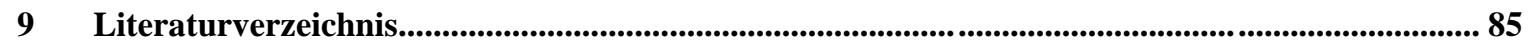




\section{Abbildungsverzeichnis}

Abbildung 2-1. Strukturformel und Extinktionsspektrum des Farbstoffs Indocyaningrün (ICG) 3

Abbildung 2-2. $\quad$ Registrierte Thermodilutionskurve und Extrapolation an den abfallenden Schenkel der Primärkurve

Abbildung 2-3. $\quad$ Registrierte Thermodilutionskurve, bei der durch spontane Temperaturschwankungen des Patienten eine Temperaturdrift auftrat ............................................................... 11

Abbildung 2-4. Mathematisches Rezirkulationsmodell und aortale Farbstoffverdünnungskurve............. 16

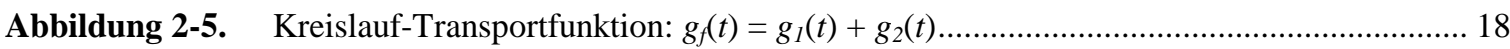

Abbildung 3-1. Zentralvenöse Bolusapplikation des Indikators in das proximale Lumen des Pulmonaliskatheters und Lage des aortalen Fiberoptik-Katheters.

Abbildung 3-2. Aufbau des verwendeten Hämoreflektometers zur Erfassung der Farbstoffdilutionskurven (nach Wietasch 1995, S. 26) .......................................................... 27

Abbildung 3-3. Pulmonal und aortal registrierte Thermodilutionskurven ........................................... 28

Abbildung 3-4. Aortal registrierte Farbstoffdilutionskurve ........................................................2 28

Abbildung 3-5. Die Extinktionen der abgenommenen Plasmaproben im Spektrumbereich zwischen 600 und $900 \mathrm{~nm}$ gegen bidestilliertes Wasser

Abbildung 3-6. Beispiel eines Extinktionskoeffizientenspektrums, das als Kalibrationsspektrum zur Berechnung der ICG-Konzentration der Blutproben zu jedem Meßzeitpunkt diente...... 31

Abbildung 3-7. Konzentrations-Zeitverlauf von ICG; Darstellung der angepaßten Modellfunktionen auf der Basis von ein- und zweikompartimentellem Eliminationsmodell............................. 32

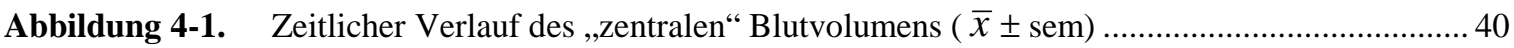

Abbildung 4-2. ,Extravaskuläres Lungenwasser“ bei den Patientengruppen $(\bar{x} \pm \operatorname{sem})$........................ 41

Abbildung 4-3. Zeitlicher Verlauf des ,zirkulierenden“ Blutvolumens ( $\bar{x} \pm$ sem) .................................42

Abbildung 4-4. Zeitlicher Verlauf des ,gesamten“ Blutvolumens ( $\bar{x} \pm$ sem $)$...................................... 43

Abbildung 5-5. Vergleich der Methoden zur Ermittlung der Plasmavolumina mittels ein- oder zweikompartimenteller Analyse $(n=60)$

Abbildung 5-6. Vergleich der Methoden zur Ermittlung der Plasmavolumina mittels spektralphotometrischer oder Einwellenlänge-Analyse $(n=80)$

Abbildung 5-7. Vergleich der Methoden zur Ermittlung der Plasmavolumina mittels Rückextrapolation auf den Nullzeitpunkt oder auf den Zeitpunkt der ersten Leberpassage $(n=82)$..............57

Abbildung 6-1. Korrelation von ,gesamtem“ und ,zirkulierendem“ Blutvolumen ..... 65

Abbildung 6-2. Vergleich der zeitlichen Verläufe vom ,gesamten“ und ,zirkulierenden“ Blutvolumen $(\bar{x} \pm$ sem)

Abbildung 6-3. Relative prozentuale Änderungen vom „,gesamten“ und ,zirkulierenden“ Blutvolumen gegenüber dem Ausgangspunkt (n.N.), $\bar{x} \pm$ sem.

Abbildung 6-4. Relative prozentuale Änderungen von ,zentralem“ und ,peripherem“ Blutvolumen gegenüber dem Ausgangspunkt (n.N.) sowie Anteil des ,zentralen“ am ,zirkulierenden“ Blutvolumen ( $\bar{x} \pm$ sem)

Abbildung 6-5. $\quad$ Relative prozentuale Änderungen von Volumen $\left(V_{d}\right)$ und Fluß $(C I)$ der Einzelkompartimente gegenüber dem Ausgangspunkt (n.N.), $\bar{x} \pm$ sem....

Abbildung 6-6. Kumulative Flüssigkeitsbilanzen der Patientengruppen $(\bar{x} \pm \mathrm{sem})$............................. 75

Abbildung 6-7. Absolute Änderungen des ,extravaskulären Gesamtwassers“ $(E V G W)$ gegenüber dem

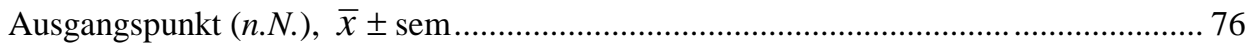

Abbildung 6-8. Absolute Änderungen des ,extravaskulären Lungenwassers“ $(E V L W)$ gegenüber dem Ausgangspunkt (n.N.), $\bar{x} \pm$ sem 


\section{Tabellenverzeichnis}

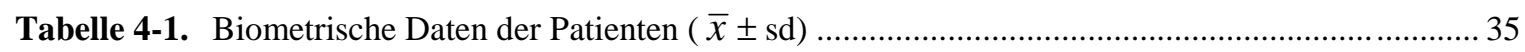

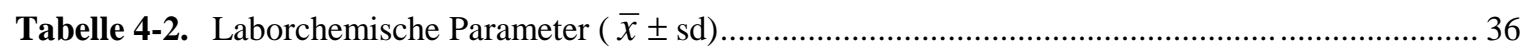

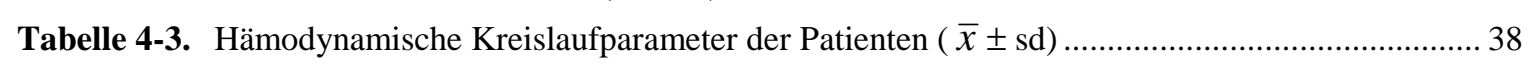

Tabelle 4-4. Kumulative Dosen applizierter Medikamente $(\bar{x} \pm \mathrm{sd})$................................................... 39

Tabelle 4-5. Ausdehnung und Durchblutung der von den Kreislauf-Transportfunktionen ermittelten Blutkompartimente und ihre Relation zum ,peripheren“ Blutvolumen $(\bar{x} \pm$ sem) ................4 44

Tabelle 4-6. Kumulative Flüssigkeitsbilanzen der Patientengruppen $(\bar{x} \pm \mathrm{sd})$................................... 45

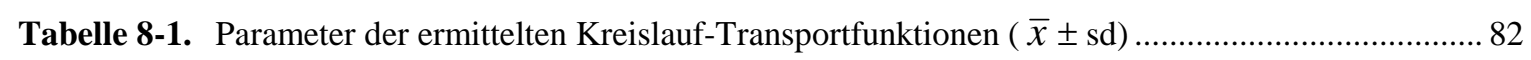

Tabelle 8-2. Einfluß der Narkoseeinleitung mit Sufentanil auf die hämodynamischen Parameter und die

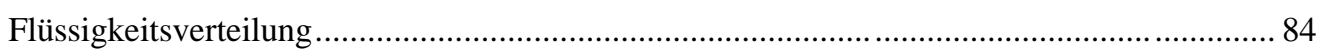




\section{Einleitung}

Im Rahmen der intensivmedizinischen Betreuung chirurgischer Patienten kommt der perioperativen Flüssigkeitstherapie eine entscheidende Bedeutung zu. Ziel der therapeutischen Maßnahmen ist, trotz teilweise großer Flüssigkeitsverluste und -verschiebungen einen adäquaten Füllungszustand des Kreislaufsystems zu gewährleisten. Insbesondere nach großen Eingriffen, wie zum Beispiel nach Operationen unter Einsatz der Herz-Lungen-Maschine, ist bei oft älteren und multimorbiden Patienten oder bei komplizierten Verläufen die körpereigene Kompensationsbreite deutlich eingeschränkt.

Während einer Operation sind die physiologischen Regulationsmechanismen zur Aufrechterhaltung der intravasalen Volumenhomöostase durch pharmakologische Einflüsse im Rahmen der Narkose beeinträchtigt (Moos und Craigo 1994; Schwinn et al. 1994). Zusätzlich wird durch den Blutkontakt mit Fremdoberflächen eine mediator-vermittelte ,allgemeine unspezifische Entzündungsreaktion“ induziert (Chenoweth et al. 1981; Kirklin et al. 1983; Boldt et al. 1986; Westaby 1987; Haeffner-Cavaillon et al. 1989; Jansen et al. 1991a; Jansen et al. 1991b; Schorn et al. 1992; Butler et al. 1993; Elliott und Finn 1993; Schorn 1995), die durch die Wirkung der aktivierten Mediatoren mit einer Störung der Integrität des Kapillarendothels und unkontrollierter Extravasation von Flüssigkeit aus dem intravaskulären in den extravaskulären Raum einhergeht (Blomqvist et al. 1990; Frostell et al. 1990; Wiener-Kronish und Broaddus 1993). Das auf dieser Weise entstandene „Ganzkörperödem“ kann sowohl indirekt durch Beeinträchtigung der nutritiven Versorgung als auch direkt durch spezifische Funktionsbeeinträchtigung schädigend wirken. Auch nach Reetablierung normaler intravasaler Volumenverhältnisse können aufgrund einer bereits erfolgten Veränderung der interstitiellen Matrix pathologische Zustände persistieren (Comper 1984). Dies kann durch eine negative Beeinflussung des Verhältnisses zwischen Sauerstoffzufuhr und -verbrauch zu einer Störung der Gewebsoxygenierung und zu einer konsekutiven Organfunktionsstörung (Chenoweth et al. 1981; Kirklin et al. 1983; Westaby 1987; Butler et al. 1993) führen.

Somit können Maßnahmen, die der Entstehung und Ausprägung des interstitiellen Ödems entgegenwirken, eine Bedeutung zukommen. In diesem Sinne ergeben sich auf folgenden unterschiedlichen Ebenen Ansätze für eine präoperative Applikation von Glucocorticoiden (Boldt et al. 1986; Westaby 1987; Butler et al. 1993; Miranda et al. 1982):

1. Verbesserung der Makro- bzw. Mikrozirkulation durch prä- und postkapilläre Vasodilatation mit Verhinderung kapillärer Stase und Thrombozytenaggregation (Piepenbrock et al. 1977; Sellevold und Jynge 1985).

2. Begrenzung der Komplement-induzierten Granulozytenaggregation (Westaby 1987; Butler et al. 1993).

3. Direkte Abdichtung der Kapillarendothelien und damit Bekämpfung einer deletären Überflutung des Interstitiums mit Flüssigkeit (Sibbald et al. 1981; Wilson 1972).

4. Verhinderung der Freisetzung aktiver biogener Amine durch Stabilisierung der Leukozyten-, Thrombozyten- und lysosomalen Membranen (Wilson 1972). 
5. Supprimierung der Interleukinproduktion und insbesondere der TNF- $\alpha$ - und $\mathrm{LTB}_{4}$-Freisetzung sowie der t-PA-Aktivität während der Reperfusionsphase des Herzens und der Lunge (Jansen et al. 1991a).

Ziel der vorliegenden Studie war zu ermitteln, inwieweit durch eine präoperative Applikation von Glucocorticoiden die mediator-vermittelte unspezifische Entzündungsreaktion supprimiert und dadurch die generalisierte Ödembildung günstig beeinflußt werden kann. Zur Bearbeitung dieses Fragenkomplexes wurden in der vorliegenden Studie koronarchirurgische Patienten doppelblind in zwei Gruppen randomisiert. Patienten der ersten Gruppe (Placebo-Gruppe) erhielten keine Glucocorticoide, die der zweiten Gruppe (Dexamethason-Gruppe) $1 \mathrm{mg} \cdot \mathrm{kg}^{-1}$ Körpergewicht Dexamethason intravenös nach Einleitung der Narkose. Beide Gruppen wurden mit Hilfe der Indikatordilutionsmethoden hinsichtlich ihres Volumenstatus nach der Narkoseeinleitung sowie in der frühen postoperativen Phase untersucht. 


\section{Theoretische Grundlagen der Indikatordilution}

Der Blutkreislauf stellt das wichtigste intrakorporale Transportsystem der Menschen dar. Die Blutströmung ermöglicht den Tansportvorgang, der in vitro und in vivo mittels verschiedener Indikatoren untersucht werden kann. Beim Indikatordilutionsverfahren werden dem strömenden Blut an einer bestimmten Stelle in möglichst kurzer Zeit definierte Indikatormengen in Form von Kälte, Wärme, Farbstoffen oder radioaktiven und leitfähigkeitsändernden Substanzen zugeführt und in „stromabwärts“ gelegenen Abschnitten die zeitlichen Konzentrationsverläufe der Indikatoren bestimmt. An der Meßstelle ergeben sich unterschiedliche Ankunftszeiten der einzelnen Indikator-Partikel abhängig von den Eigenschaften der Indikatoren, dem Gefäßsystem und dem Blutfluß. Demzufolge resultiert eine zunehmende Verdünnung und zeitliche Dispersion der Indikatoren, die am Meßort zur Registrierung der typischen Indikatordilutionskurven ( $\mathrm{vgl}$. Abbildung 3-3 und Abbildung 3-4) mit asymmetrischen Formen führt (Lassen und Perl 1979; Zierler 1962a; Zierler 1962b).

\subsection{Indikatoren und Auswertungsprinzipien in der Dilutionstechnik}

Bei gleichzeitiger Anwendung von Indikatoren mit unterschiedlichen physikochemischen Eigenschaften können aus den registrierten Indikatorverdünnungskurven verschiedene Verteilungsräume erfaßt werden. Zur Ermittlung dieser Körpervolumina und Kreislaufkompartimente, die in erster Linie durch die Diffusion der Indikatoren bestimmt werden, wurden in der vorliegenden Studie ein intravasal verbleibender (der Farbstoff Indocyaningrün, ICG) und ein diffusiver Indikator (die Kälte) gewählt.
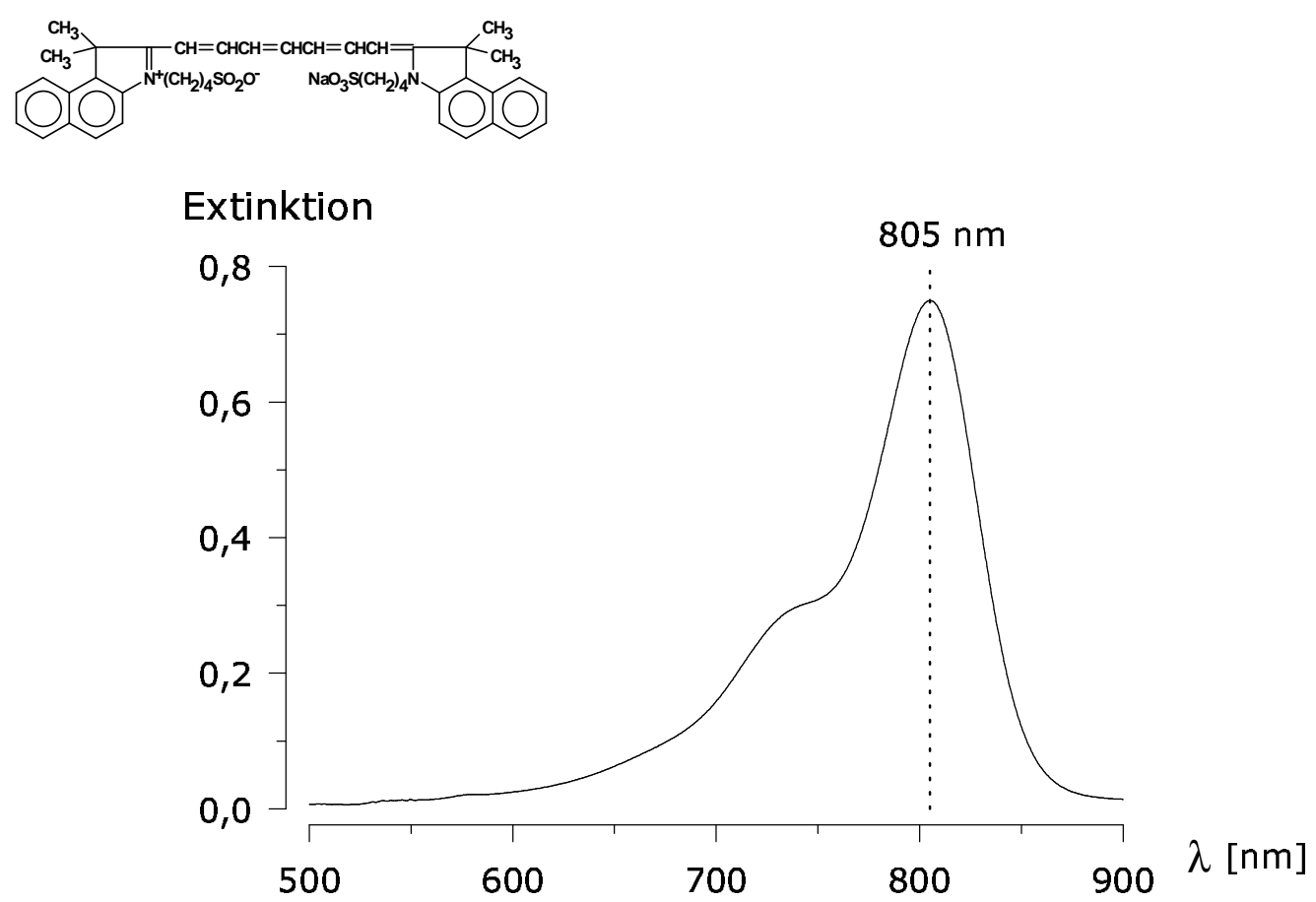

Abbildung 2-1. Strukturformel und Extinktionsspektrum des Farbstoffs Indocyaningrün (ICG) $\lambda=$ Wellenlänge in $\mathrm{nm}$; Extinktionsmaximum bei $805 \mathrm{~nm}$ 
Die klinische Anwendung von Indocyaningrün (ICG) als Indikator bei Dilutionstechniken wurde erstmals von Fox und Mitarbeitern (Fox et al. 1957) vor allem zur Herzzeitvolumenmessung und zur Abschätzung der Leberdurchblutung beschrieben. 1968 setzten Bradley und Barr ICG zur Blutvolumenbestimmung ein (Bradley und Barr 1968). Es handelt sich dabei um einen anionischen Tricarbocyanin-Farbstoff (s. Abbildung 2-1), der infolge seiner Wasserlöslichkeit und guten Verträglichkeit für sterile intravasale Injektionen geeignet ist. ICG ist nicht toxisch und kann nur in äußerst seltenen Fällen anaphylaktoide Reaktionen hervorrufen (Benya et al. 1989; Speich et al. 1988). Der in vivo inerte Farbstoff wird nach der Injektion nahezu vollständig an die Plasmaproteine gebunden und wird daher zu den streng intravasalen Indikatoren gerechnet (Fox und Wood 1960). Die Elimination von ICG erfolgt mit einer Halbwertzeit von 5 bis 10 Minuten ausschließlich über die Leber (Paumgartner 1975).

Das Absorptionsmaximum des in Wasser gelösten ICGs liegt bei der Wellenlänge $780 \mathrm{~nm}$, verschiebt sich jedoch nach intravasaler Injektion und Bindung an die Plasmaeiweißfraktion (hauptsächlich an $\alpha_{l}$-Lipoproteine) bis auf $810 \mathrm{~nm}$ (Baker 1966). Bei dem verwendeten $I C G^{1}$ lag das Absorptionsmaximum bei der Wellenlänge $805 \mathrm{~nm}$ (s. Abbildung 2-1), bei der auch ein isosbestischer Punkt ${ }^{2}$ für das Hämoglobin vorliegt (Landsman et al. 1976).

Der nichtmolekulare Indikator Kälte ist hochdiffusiv und verläßt nach der Applikation den Intravasalraum. Er verteilt sich im umliegenden Gewebe und eignet sich somit zur Erfassung der intra- samt extravaskulären Flüssigkeitsräume.

Die Analyse der Indikatordilutionskinetiken zur Bestimmung von Blutflüssen und Messung von Flüssigkeitsräumen unterliegt je nach Fragestellung unterschiedlichen Prinzipien (Lassen und Perl 1979; Grodins 1962; Zierler 1962a; Zierler 1962b), die in den folgenden Kapiteln 2.1.1 und 2.1.2 erläutert werden.

\subsubsection{Das Prinzip der Massenerhaltung}

\subsubsection{Flußmessungen nach dem Prinzip der Massenerhaltung}

Nach dem Massenerhaltungsprinzip muß eine bestimmte Indikatormenge $\left(m_{0}\right)$, die in den Blutstrom appliziert wird $\left(m_{\text {rein }}\right)$, mengenmäßig stromabwärts wieder erscheinen $\left(m_{\text {raus }}\right)$.

$$
m_{0}=m_{\text {rein }}=m_{\text {raus }}
$$

Nach einer Bolusapplikation des Indikators in die Blutbahn resultiert am Meßort eine Indikatordilutionskurve mit der typischen linksschiefen Form (s. z.B. Abbildung 3-3). Betrachtet man die am Meßort pro Zeiteinheit vorbeifließende Indikatormenge, kann die sogenannte Indikatorflußdichte $\left(\dot{m}_{\text {raus }}(t)\right)$ definiert werden. Sie ergibt sich aus dem Produkt vom Fluß $(\dot{Q}(t))$ und

${ }^{1}$ ICG-Pulsion $^{\circledR}$, Pulsion Medical Systems, München, BRD oder

Cardiogreen ${ }^{\circledR}$, Paesel+Lorei GmbH \& Co, Frankfurt, BRD

${ }^{2}$ Bei dieser Wellenlänge ist die Lichtabsorption des Hämoglobins unabhängig von der Sauerstoffbindung. 
Konzentration des Indikators $(c(t))$ zum jeweiligen Zeitpunkt:

$$
\dot{m}_{\text {raus }}(t)=\dot{Q}(t) \cdot c(t)
$$

Demnach kann die Gesamtindikatormenge $m_{\text {raus }}$, die nach der Bolusapplikation den Meßort passiert, aus dem Zeitintegral der Indikatorflußdichte bestimmt werden:

$$
m_{\text {raus }}=\int_{t=0}^{\infty} \dot{m}_{\text {raus }}(t) d t=\int_{t=0}^{\infty} \dot{Q}(t) \cdot c(t) d t
$$

Unter der Annahme, daß der Fluß über die Zeit als konstant angesehen werden kann, kann der Fluß vor das Integral geschrieben und aus dem Verhältnis der zugeführten Indikatormenge zur Fläche unter der Indikatorverdünnungskurve (wird als $A U C^{3}$ bezeichnet und entspricht dem Integral $\int_{t=0}^{\infty} c(t) d t, s$. Abbildung 3-3) berechnet werden:

Gleichung 2-1. Stewart-Henriques-Hamilton-Verfahren:

$$
m_{0}=m_{\text {raus }}=\dot{Q} \cdot \int_{t=0}^{\infty} c(t) d t \Rightarrow \dot{Q}=\frac{m_{0}}{\int_{t=0}^{\infty} c(t) d t}
$$

\subsubsection{Messung des Verteilungsvolumens nach dem Prinzip der Massenerhaltung}

Nach seiner Definition ist das Verteilungsvolumen (,,volume of distribution“", $V_{d}$ ) eines Indikators ein Proportionalitätsfaktor zwischen der im Organismus applizierten Menge $m_{0}$ des Indikators und seiner Plasmakonzentration $c_{0}$. Es gilt die Beziehung

\section{Gleichung 2-2}

$$
\begin{aligned}
& m_{0}=V_{d} \cdot c_{0} \Rightarrow V_{d}=\frac{m_{0}}{c_{0}} \\
& m_{0} \quad=\text { applizierte Indikatormenge } \\
& V_{d} \quad=\text { Proportionalitätsfaktor, entspricht dem Veteilungsvolumen (,, volume of distribution “) } \\
& c_{0} \quad=\text { virtuelle Indikatorkonzentration zum Zeitpunkt } t=0
\end{aligned}
$$

Dieser Proportionalitätsfaktor zwischen der Menge (in $m g$ ) und der Konzentration (in $m g \cdot l^{-1}$ ) hat die Dimension eines Volumens, nämlich Liter. Basierend auf diesem einfachen Zusammenhang der Gleichung 2-2 kann ein unbekanntes Verteilungsvolumen mit Hilfe einer bekannten Indikatormenge durch Konzentrationsmessungen bestimmt werden.

${ }^{3} A U C$ ist die Abkürzung von ,area under curve“ und entspricht der Fläche unter der Indikatorverdünnungskurve. 


\subsubsection{Das Prinzip der Indikatordispersion}

$\mathrm{Zu}$ unterscheiden vom Prinzip der Massenerhaltung und dem Stewart-Henriques-HamiltonVerfahren ist die Messung der Verteilungvolumina auf der Basis der Transitzeiten ( $m t t$ 's) eines Indikators. Aufgrund makrodispersiver Mechanismen durchlaufen die einzelnen Partikel des an einer Stelle des Gefäßsystems eingebrachten Indikators einen bestimmten Gefäßabschnitt in unterschiedlichen Zeiten (Meier und Zierler 1954). Wenn man die am Meßort resultierende Konzentrationszeitkurve (oder Indikatorverdünnungskurve) auch als Transitzeitenverteilung der einzelnen Indikator-Partikel betrachtet, entspricht die Indikatorkonzentration $\mathrm{zu}$ einem bestimmten Zeitpunkt der Anzahl der Partikel zu diesem Zeitpunkt (Lassen und Perl 1979). Die mittlere Transitzeit des Indikators durch den betrachteten Gefäßabschnitt ( $m t t)$ berechnet sich demzufolge nach der Formel:

$$
\begin{aligned}
& m t t=\frac{c_{1} \cdot t_{1}+c_{2} \cdot t_{2}+\ldots+c_{n} \cdot t_{n}}{c_{1}+c_{2}+\ldots+c_{n}}=\frac{\sum_{i=1}^{n} c_{i} \cdot t_{i}}{\sum_{i=1}^{n} c_{i}} \\
& c_{i} \quad=\text { Indikatorkonzentration zum Zeitpunkt } t_{i}
\end{aligned}
$$

Unter Berücksichtigung der gesamten Dilutionskurve ergibt sich aus dem Zeitintegral:

\section{Gleichung 2-3}

$$
\begin{aligned}
& m t t= \frac{\int_{t=0}^{\infty} c(t) \cdot t d t}{\int_{t=0}^{\infty} c(t) d t} \\
& c(t) \quad=\text { Indikatorkonzentration zum Zeitpunkt } t
\end{aligned}
$$

Wenn der Fluß $(\dot{Q})$ und die mittlere Transitzeit des Indikators ( $m t t)$ als Meßgrößen vorliegen, kann die folgende grundlegende Beziehung zur Bestimmung von Verteilungsvolumina genutzt werden (Lassen und Perl 1979):

\section{Gleichung 2-4}

$$
\begin{aligned}
& \dot{Q}=\frac{V_{d}}{m t t} \Rightarrow V_{d}=\dot{Q} \cdot m t t \\
& \dot{Q} \quad=\text { Fluß des Tranportmediums } \\
& V_{d}=\text { Verteilungsvolumen des Indikators } \\
& m t t \quad=\text { mittlere Transitzeit des Indikators }
\end{aligned}
$$




\subsubsection{Die stochastische Systemanalyse}

Die theoretischen Grundlagen der „stochastischen Systemanalyse“, die im Bereich der Elektrotechnik breite Anwendung findet, wurden erstmals von Stephenson (1948) zur Beschreibung von Indikatorverdünnungskurven und später von Meier und Zierler (1954) zur Untersuchung des organspezifischen Dispersionvorganges ausgeführt. Ähnlich wie StromSpannungskurven bei der Passage eines elektronischen Filters, ergibt sich der KonzentrationsZeitverlauf, mit dem ein Indikator nach Durchgang eines Kreislaufabschnittes oder -organs am Registrierort erscheint, aus dem Konzentrations-Zeitverlauf am Eingang sowie aus der spezifischen Dispersion, der der Indikator beim Durchwandern der Gefäße unterworfen wurde.

\subsubsection{Grundlagen}

Charakteristische Eigenschaften eines unbekannten Systems (,black box“) können mittels der stochastischen Systemanalyse aus der Kenntnis der Eingangs- und Ausgangsfunktion abgeleitet werden. Notwendige Voraussetzung für die Anwendbarkeit dieses Ansatzes ist, daß sich das untersuchte System stationär und linear verhält.

Ein stationäres System liegt vor, wenn während des Untersuchungszeitraumes eine Wiederholung der gleichen Eingangsfunktion, zumindest beim Vorliegen eines Fließgleichgewichtes im Sinne eines „steady state“, zur gleichen Systemantwort führt. Im Rahmen der vorliegenden Untersuchungen darf annäherungsweise trotz der rhythmischen Herzaktivität von stationären Verhältnissen ausgegangen werden, denn die zeitliche Dispersion des Indikators überdauert mehrere Herzschläge und somit wird die resultierende Indikatorverdünnungskurve bezüglich des Herzzyklus unabhängig vom Applikationszeitpunkt des Indikators (Bassingthwaighte et al. 1966a; Bassingthwaighte et al. 1966b; Knopp et al. 1976; Meier und Zierler 1954; Zierler 1962b).

Wenn die Überlagerung und Superposition von Eingangsfunktionen zu einer Überlagerung und Superposition der zugehörigen Ausgangsfunktionen führt, liegt ein lineares Systemverhalten vor. Die Bedingung der Linearität wird auch von solchen Indikatoren erfüllt, die innerhalb eines untersuchten Kreislaufsystems zum Teil eliminiert werden, da der lineare Zusammenhang zwischen Eingangs- und Ausgangssignal erhalten bleibt, sofern der Indikatorverlust proportional zur Indikatorkonzentration ist. Daraus folgt, daß für die Anwendung der stochastischen Systemanalyse nicht die gesamte Indikatormenge erfaßt werden muß. Die Registrierung des Konzentrations-Zeitverlaufs in einer repräsentativen zuführenden Arterie und in einer repräsentativen abführenden Vene ist dementsprechend ausreichend (Lassen und Perl 1979).

\subsubsection{Das Faltungsintegral}

Wenn sich ein System ausreichend stationär und linear verhält, läßt sich der Zusammenhang zwischen dem Signal am Eingang des untersuchten Systems und an seinem Ausgang durch ein sog. Faltungsintegral beschreiben: 


\section{Gleichung 2-5}

$$
\begin{array}{ll}
c_{\text {out }}(t) & =\int_{0}^{t} c_{\text {in }}(t-u) \cdot g(u) d u \\
c_{\text {out }}(t) & =\text { Ausgangssignal } \\
c_{\text {in }}(t) & =\text { Eingangssignal } \\
g(t) & =\text { Transportfunktion } \\
u & =\text { Integrationsvariable der Zeit }
\end{array}
$$

Die Funktion $g(t)$, die als Gewichtsfunktion bezeichnet wird, drückt die charakteristischen Eigenschaften des unbekannten untersuchten Systems aus. Bei Kenntnis dieser Funktion und einer beliebigen Eingangsfunktion $c_{i n}(t)$ läßt sich die am Organausgang zu erwartende Systemantwort $c_{\text {out }}(t)$ durch Faltung $(*)$ nach Gleichung 2-5 berechnen. Vereinfacht ist

$$
c_{\text {out }}(t)=c_{\text {in }}(t) * g(t) .
$$

Für den Sonderfall einer Dirac'schen Impulsfunktion $i(t)$ am Organeingang resultiert am Organausgang eine Impulsantwort, die in idealer Weise die charakteristischen Eigenschaften des Organs beschreiben würde und mit der Gewichtsfunktion des Systems identisch wäre:

$$
\begin{aligned}
& c_{\text {out }}(t)=i(t) * g(t)=g(t) \\
& \left.\begin{array}{c}
i(t)=0 \text { für } t \neq 0 \\
i(t) \neq 0 \text { für } t=0 \\
\int_{-\infty}^{\infty} i(t) d t=1
\end{array}\right\} \text { Dirac'sche Impulsfunktion }
\end{aligned}
$$

Bassingthwaighte führte 1967 den Begriff der „Transportfunktion“ ein, der inhaltlich mit dem vorgenannten Begriff „Gewichtsfunktion“ identisch ist. Bei der Indikatordilution entspricht die Transportfunktion eines Systems derjenigen Funktion, die bei Applikation des Indikators auf der arteriellen Seite in Form einer idealen Dirac-Anregung als venöse Antwort resultieren würde. Demzufolge kann die Transportfunktion auch als Wahrscheinlichkeitsdichtefunktion der Indikatorpassagezeiten interpretiert werden und wird auch als Transitzeitenverteilung bezeichnet (Bassingthwaighte und Ackerman 1967). Die mittlere Transitzeit entspricht dann dem Erwartungswert dieser Transitzeitenverteilung und ergibt sich aus der Formel:

\section{Gleichung 2-6}

$$
m t t=\int_{0}^{\infty} t h_{k}(t) d t
$$

\subsubsection{Die Entfaltung von Indikatorverdünnungskurven}

Eine impulsartige Applikation des Indikators und damit eine direkte Bestimmung der organspezifischen Transportfunktion ist jedoch in vivo nicht realisierbar. Bei Kenntnis des Eingangsund Ausgangs-Konzentrations-Zeitverlaufes ist es jedoch möglich, durch eine Umkehrung des 
Faltungsintegrals der Gleichung 2-5 (sog. „Entfaltung“) die für den Dispersionsvorgang charakteristische Transportfunktion zu berechnen.

Zur Entfaltung wird ein nicht-lineares Anpassungsverfahren eingesetzt, welches auf dem Prinzip der kleinsten Abstandsquadrate (Deuflhard und Apostolescu 1980) basiert. Unter der Voraussetzung, daß die gesuchte Transportfunktion durch eine mathematische Modellfunktion beschreibar ist, werden initiale Schätzwerte der Parameter dieser für das untersuchte System geeigneten Modellfunktion solange iterativ verändert, bis eine optimale Übereinstimmung zwischen der gemessenen Organausgangsfunktion und der durch Faltung (Gleichung 2-5) mathematisch simulierten Ausgangsfunktion erreicht wurde.

\subsection{Ermittlung des Herzzeitvolumens}

Auf der Basis des Massenerhaltungsprinzips wurde das Herzzeitvolumen $(H Z V)$ nach dem klassischen Stewart-Henriques-Hamilton-Verfahren (vgl. Gleichung 2-1) bestimmt, jedoch erfolgte seine Berechnung nur aus den pulmonal und aortal registrierten Thermodilutionskurven. Das $H Z V$ könnte theoretisch auf ähnlicher Weise aus den Farbstoffdilutionskurven bestimmt werden, eine exakte Kalibration der Farbstoffkurven zeigte sich aber in der Praxis problematisch, trotz der hohen Linearität der fiberoptischen Farbstoffmessungen (Wietasch 1995). Die applizierte Indikator-Kältemenge $\left(m_{0}\right)$ berechnet sich in Abhängigkeit von der Differenz der Blut- und Injektattemperatur $\left(T_{B l u t}-T_{I n j}\right)$ und vom tatsächlichen Injektatvolumen $\left(V_{I n j}-V_{T}\right)$ nach der folgenden Formel:

$$
m_{0}=\left(T_{B l u t}-T_{I n j}\right) \cdot\left(V_{I n j}-V_{T}\right)
$$

Für das HZV ergibt sich dann anhand der Gleichung 2-1 folgende Formel:

\section{Gleichung 2-7}

$$
\begin{aligned}
H Z V & =\frac{m_{0}}{\int_{0}^{\infty}-\Delta T(t) d t} \cdot k_{\text {spez }}=\frac{\left(T_{\text {Blut }}-T_{\text {Inj }}\right) \cdot\left(V_{\text {Inj }}-V_{T}\right)}{\int_{0}^{\infty}-\Delta T(t) d t} \cdot k_{\text {spez }} \\
T_{B l u t}, T_{\text {Inj }} & =\text { Blut- und Injektattemperatur } \\
V_{\text {Inj }}, V_{T} & =\text { Injektat- und Kathetertotraumvolumen } \\
-\Delta T(t) & =\text { Temperatur-Zeitverlauf } \\
k_{\text {spez }} & =\text { Konstante }
\end{aligned}
$$

Die Unterschiede von Dichte und spezifischer Wärmekapazität zwischen Injektat und Blut müssen berücksichtigt werden und werden durch die Kalibrationskonstante $\left(k_{\text {spez }}\right)$ in die Berechnung des HZVs einbezogen. Für 0,9\%ige NaCl-Lösung beträgt sie 1,102 und ist über einen weiten Bereich Hämatokrit-unabhängig.

Die Abgrenzung der Primärkurve und die Berechnung der Fläche unter der Thermodilutionskurve (oder AUC, entspricht dem Integral in der Gleichung 2-7) stellt bei der $H Z V$-Bestimmung mittels Indikatordilution ein grundsätzliches Problem dar (s. Abbildung 2-2). Ursächlich sind nach Bolusapplikation intravasaler Indikatoren am Registrierort zusätzlich erscheinende rezirkulations- 
bedingte Indikatoranteile. Zur korrekten Ermittlung der $A U C$ ist jedoch unter Berücksichtigung des Prinzips der Indikatormassenerhaltung zu fordern, daß keine rezirkulierenden Indikatoranteile miterfaßt werden, da dies eine Überschätzung des Temperaturzeitintegrals und somit eine Unterschätzung des resultierenden Stromzeitvolumens zur Folge hätte. Hamilton et al. stellten bereits 1928 fest, daß dies durch eine monoexponentielle Extrapolation des abfallenden Schenkels der Thermodilutionskurve gewährleistet wird, wobei man davon ausgeht, daß die Kurve in Abwesenheit einer Rezirkulation einen exponentiellen Abfall aufweisen würde. Die klassische monoexponentielle Extrapolation wird an den abfallenden Kurvenschenkel meist zwischen $70 \%$ und $33 \%$ des jeweiligen Kältemaximums angelegt (Wessel et al. 1971; Pearce und Beazell 1966). Bei den vorliegenden Untersuchungen erfolgte die Extrapolation der Thermodilutionskurve jedoch nicht zwischen zwei festgelegten Grenzen ( $v$ gl. Abbildung 2-2), sondern es wurde eine Extrapolation durch individuelle nichtlineare Anpassung der abfallenden Schenkel der Thermodilutionskurven an Modellfunktionen vorgenommen, die imstande sind, die charakteristische Form von Verdünnungskurven nachzubilden (Weyland et al. 1995; Mehlhorn et al. 1990). Im Vergleich zur üblichen Extrapolation weist die Anpassung an Modellfunktionen den Vorteil auf, daß die gesamte Kurve und damit mehr Informationen der individuellen Verdünnungskurve ausgenutzt werden.

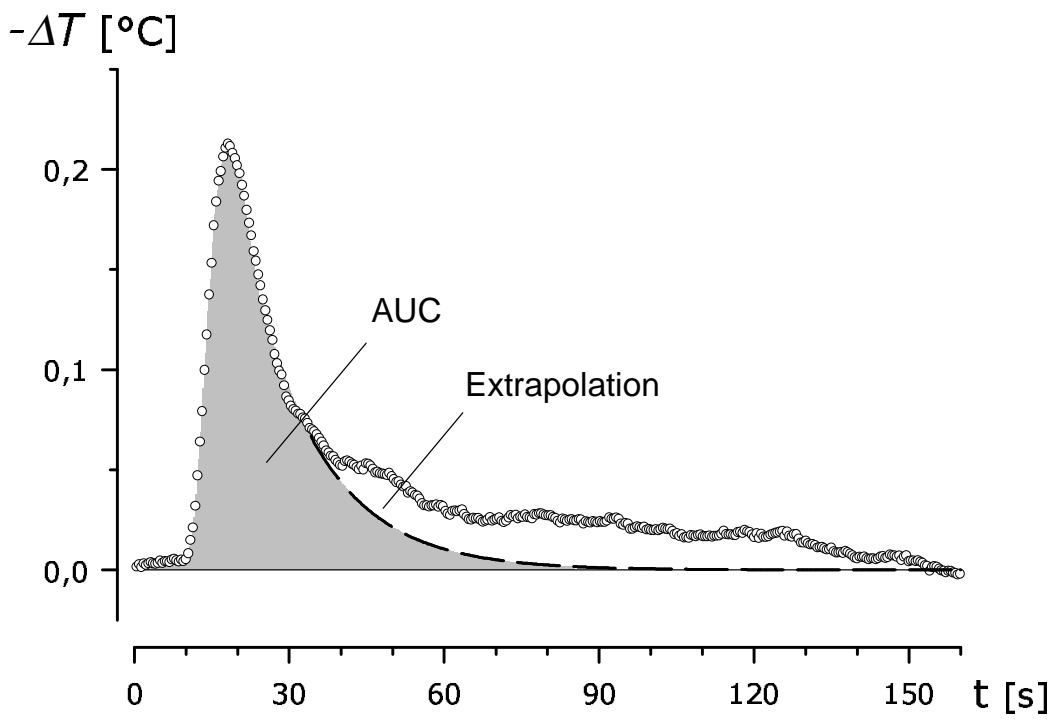

Abbildung 2-2. Registrierte Thermodilutionskurve und Extrapolation an den abfallenden Schenkel der Primärkurve

$A U C=$ Fläche unter der Primärkurve (,,area under curve“)

Eine weitere Fehlerquelle zur Bestimmung des Herzzeitvolumens stellen spontane Temperaturschwankungen des Patienten dar, wie sie zum Beispiel bei Situationen mit hämodynamischer Kreislaufinstabilität oder iatrogen durch während der Aufzeichnung rasch einlaufende Infusionen verursacht werden. Diese Temperaturschwankungen machen sich als Neigung der Basislinie (,drift“) der aufgezeichneten Thermodilutionskurven bemerkbar (s. Abbildung 2-3). Bei den 
vorliegenden Messungen wurden Thermodilutionskurven mit erkennbarer Abweichung entsprechend korrigiert (vgl. Abbildung 2-2 und Abbildung 2-3), unter der Voraussetzung, daß eine Temperaturabweichung über den Meßzeitraum von vier Minuten linear verläuft (Wietasch 1995). Andernfalls wird bei der Abgrenzung der Primärkurve die Fläche unter der Kurve durch die aufgetretene Neigung der Temperatur-Basislinie zu klein eingeschätzt, wodurch eine Überschätzung des $H Z V s$ resultiert. Hierzu ist jedoch anzumerken, daß eine Messung unter stabilen Bedingungen ,steady state“ günstiger ist als eine nachträgliche mathematische Korrektur der Dilutionskurven.

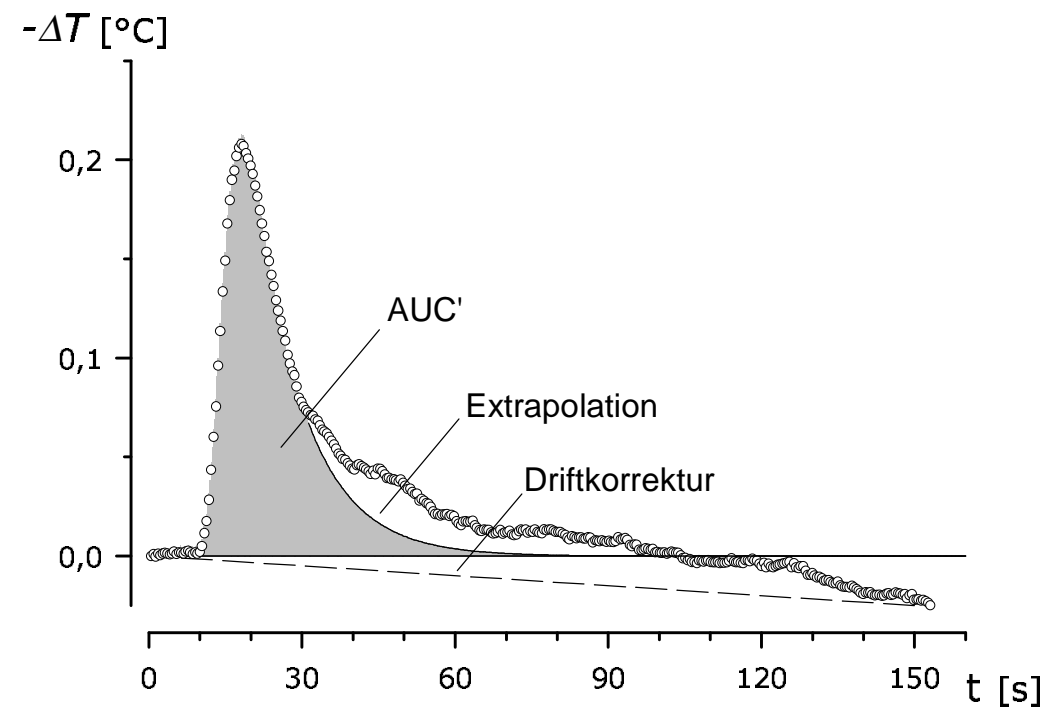

Abbildung 2-3. Registrierte Thermodilutionskurve, bei der durch spontane Temperaturschwankungen des Patienten eine Temperaturdrift auftrat

$A U C^{\prime}=$ Fläche unter der Dilutionskurve mit drift (,,area under curve“ $)$

Für die weiteren Berechnungen wurde zu jeder Messung der Mittelwert aus pulmonal und aortal registriertem $H Z V$ benutzt (Wessel et al. 1971).

\section{3 „Zentrales thermoakzessibles“ Volumen}

Aufgrund seiner ausgeprägten Neigung zur Diffusion äquilibriert sich der Indikator Kälte nach zentralvenöser Applikation im rechten Vorhof während der Lungenpassage rasch und vollständig mit dem Lungengewebe. Somit wird der zugehörige intra- und extravaskuläre Raum zusammen zwischen Injektions- und Registrierort erfaßt und als Verteilungsvolumen des Indikators Kälte oder ,zentrales thermoakzessibles“ Volumen (ZTV) bezeichnet.

Nach der konventionellen Methode errechnet sich das ZTV aus dem Produkt von HZV (Gleichung 2-7) und der mittleren Transitzeit des Indikators Kälte $\left(m t t_{k}\right)$, die sich aus der monoexponentiell extrapolierten aortalen Thermodilutionskurve gemäß Gleichung 2-3 berechnen läßt (Kreuzfelder 
et al. 1988; Lewis et al. 1979; Lewis et al. 1982; Pearce und Beazell 1966). Aufgrund aber einer durch diese Methode inkompletten Elimination der rezirkulierenden Kälteanteile wird das ZTV und demzufolge das EVLW (Gleichung 2-13) erheblich überschätzt (Mehlhorn et al. 1990; Böck et al. 1988).

Damit das berechnete ZTV unabhängig von Indikatorrezirkulation und Injektionsdauer ist, wurde im Rahmen der vorliegenden Studie eine modifizierte Meßtechnik zur Berechnung der Transitzeiten angewendet (Mehlhorn et al. 1990; Böck et al. 1988). Hierbei werden die Konzentrations-Zeitverläufe der Kälte nicht nur in der Aorta, sondern simultan zusätzlich in der Arteria pulmonalis aufgezeichnet. Unter Annahme, daß die Bedingungen der Stationarität und Linearität als ausreichend erfüllt sind (vgl. Abschnitt 2.1.3.1), läßt sich der Zusammenhang zwischen Konzentrations-Zeitverlauf am Eingang (Arteria pulmonalis, $c_{k-a p}(t)$ ) des untersuchten Kreislaufabschnittes, hier der pulmonalen Strombahn, und an seinem Ausgang (Aorta, $c_{k-a o}(t)$ ) durch ein Faltungsintegral beschreiben (Bassingthwaighte et al. 1966b; Bassingthwaighte und Ackerman 1967; Knopp et al. 1976; Lassen und Perl 1979; Zierler 1962b):

\section{Gleichung 2-8}

$$
\begin{array}{ll}
c_{k-a o}(t) & =\int_{0}^{t} h_{k}(t-u) c_{k-a p}(u) d u \\
c_{k-a o}(t) & =\text { Konzentrations-Zeitverlauf der Kälte in der Aorta } \\
c_{k-a p}(t) & =\text { Konzentrations-Zeitverlauf der Kälte in der Arteria pulmonalis } \\
h_{k}(t) & =\text { Kältetransportfunktion der Lunge } \\
u & =\text { Integrationsvariable }
\end{array}
$$

Nach einer impulsartigen Indikatorapplikation am Lungeneingang, würde am Organausgang durch Faltung nach Gleichung 2-8 eine Impulsantwort resultieren, die in idealer Weise die Indikatorpassage durch die Lunge beschreiben würde und mit der spezifischen pulmonalen Kältetransportfunktion $h_{\mathrm{k}}(t)$ identisch wäre. Eine solche impulsartige Applikation des Indikators und damit eine direkte Bestimmung der Kältetransportfunktion ist jedoch in vivo nicht realisierbar. Damit $h_{\mathrm{k}}(t)$ berechnet werden kann, muß zusätzlich der Konzentrations-Zeitverlauf der Kälte am Organeingang $\left(c_{k-a p}(t)\right)$ gemessen werden und das inverse Problem der Gleichung 2-8 durch Entfaltung der registrierten Dilutionskurven gelöst werden (vgl. Abschnitt 2.1.3.3).

Zur Entfaltung wurde ein nicht-lineares Anpassungsverfahren eingesetzt, welches auf dem Prinzip der kleinsten Abstandsquadrate (Deuflhard und Apostolescu 1980) basiert und die Parameter einer für die pulmonalen Transportfunktionen geeigneten Modellfunktion ermittelt. Initiale Schätzwerte dieser Parameter wurden solange iterativ verändert, bis eine optimale Übereinstimmung zwischen der gemessenen Organausgangsfunktion und der durch Faltung (Gleichung 2-8) mathematisch simulierten aortalen Indikatorkinetik erreicht wurde.

Bei den vorliegenden Messungen konnte die von Bassingthwaighte dargestellte ,lagged normal density"-Modellfunktion $f(t)$, die ihrerseits durch Faltung $(*)$ einer Gau $\beta$-Normalverteilung $h_{l}(t)$ 
mit einer Exponentialfunktion $h_{2}(t)$ resultiert, am besten die pulmonale Transportfunktion simulieren (Bassingthwaighte et al. 1966a). Dementsprechend ergibt sich $f(t)$ aus:

\section{Gleichung 2-9}

$$
\begin{gathered}
f(t, \mu, \sigma, \tau)=h_{1}(t, \mu, \sigma) * h_{2}(t, \tau) \\
h_{1}(t, \mu, \sigma)=\frac{1}{\sigma \cdot \sqrt{2 \pi}} \cdot \exp \left(-\frac{(t-\mu)^{2}}{2 \sigma^{2}}\right) \\
\mu=\text { Erwartungswert der Gauß-Normalverteilung } \\
\sigma=\text { Standardabweichung der Gauß-Normalverteilung } \\
h_{2}(t, \tau)=\frac{1}{\tau} \cdot \exp \left(-\frac{t}{\tau}\right) \\
\tau=\text { Zeitkonstante der Exponentialfunktion }
\end{gathered}
$$

Die mittlere Transitzeit der ,lagged normal density“-Funktion berechnet sich zu (Bassingthwaighte et al. 1966a):

$$
m t t_{k-z e n t}^{\prime}=\mu+\tau
$$

ZTV ergibt sich dann gemäß Gleichung 2-4 aus dem Produkt der mittleren Transitzeit der Modellfunktion und dem HZV (Gleichung 2-7):

\section{Gleichung 2-10}

$$
\begin{aligned}
Z T V & =H Z V \cdot m t t_{k-z e n t}^{\prime} \\
m t t^{\prime} \text {-zent } & =\text { mittlere Transitzeit der ,lagged normal density"-Modellfunktion }
\end{aligned}
$$

\section{4 „Zentrales“ Blutvolumen}

Nach der Definition von Gauer (1972) entspricht das „zentrale“ Blutvolumen $\left(V_{\text {d-zent }}\right)$ dem Blutvolumen im kleinen Kreislauf, das sich zwischen der Pulmonal- und Aortenklappe befindet.

Eine Registrierung der Farbstoffdilutionskurve in der Arteria pulmonalis wurde im Rahmen der vorliegenden Studie nicht durchgeführt. Anders als bei der Bestimmung vom ZTV (vgl. Abschnitt 2.3) konnte von Böck et al. (1988) und Mehlhorn et al. (1990) in früheren Untersuchungen gezeigt werden, daß eine sehr gute Übereinstimmung zwischen den mittels monoexponentieller Extrapolation versus Entfaltung der Farbstoffdilutionskurven berechneten Werten für das $V_{\text {d-zent }}$ bestand. Durch die konventionelle Extrapolation können die Rezirkulationseffekte offenbar nahezu vollständig eliminiert werden, da eine Rezirkulation von $I C G$ erst sehr spät erfolgt.

Zur Berechnung von $V_{d \text {-zent }}$ erfolgte eine logarithmische Darstellung der abfallenden Schenkel zwischen $70 \%$ und $33 \%$ des Konzentrationsmaximums der Dilutionskurven von Kälte in der Arteria pulmonalis und von ICG in der Aorta. Durch Extrapolation der dabei entstehenden Geraden ergaben sich die Primärkurven, d.h. die Kurven, die ohne Rezirkulationseffekte 
entstehen würden. Aus der extrapolierten pulmonalen Thermodilutionskurve und aortalen Farbstoffverdünnungskurve konnten dann gemäß Gleichung 2-3 die mittleren Transitzeiten für Kälte ( $\left.m t t_{k-a p}\right)$ und $I C G\left(m t_{f-a o}\right)$ berechnet werden.

Die mittlere Transitzeit des Farbstoffindikators zwischen Arteria pulmonalis und Aorta $\left(m t_{f-z e n t}\right)$ berechnet sich anschließend nach der Formel:

\section{Gleichung 2-11}

$$
\begin{aligned}
& m t t_{f-z e n t}=\left(t_{0_{f-a o}}+m t t_{f-a o}\right)-\left(t_{0_{f-a p}}+m t t_{f-a p}\right) \\
& \cong\left(t_{0_{f-a o}}+m t t_{f-a o}\right)-\left(t_{0_{k-a p}}+m t t_{k-a p}\right) \\
& =\text { mittlere Transitzeit von } I C G \text { zwischen Art. pulmonalis und Aorta } \\
& \begin{array}{ll}
m t t_{f-z e n t} & \text { Erscheinungszeitpunkte der Indikatordilutionskurven } \\
t_{0 f-a o}, t_{0 f-a p}, t_{0 k-a p} & =\text { mittlere Transitzeiten aus den verschiedenen Indikatordilutionskurven } \\
m t t_{f-a o}, m t t_{f-a p}, m t t_{k-a p} &
\end{array}
\end{aligned}
$$

Böck et al. (1988) und Mehlhorn et al. (1990) stellten fest, daß das zeitliche Versetzen der pulmonalen Thermodilutionskurven gegenüber den korrespondierenden Farbstoffverdünnungskurven als Ausdruck eines größeren präpulmonalen Verteilungsvolumens des Indikators Kälte als das von $I C G$ bestand. Dies erklärt sich dadurch, daß ein Wärmeaustausch des diffusiven Indikators mit Wandstrukturen des rechten Herzens stattfindet. Nach Subtraktion der Transitzeiten der beiden Indikatoren zwischen Injektionsort und Arteria pulmonalis ließ sich dieses „thermoakzessible“ Verteilungsvolumen $\left(T V_{k-p r a ̈}\right)$ des rechten Herzens berechnen und betrug ca. $1,36 \mathrm{ml} \cdot \mathrm{kg}^{-1}$ Körpergewicht.

Das „zentrale“ Blutvolumen läßt sich unter Berücksichtigung des $T V_{k-p r a ̈ ~}$ anhand der Gleichung 2-4 berechnen:

\section{Gleichung 2-12}

$$
\begin{aligned}
& V_{d-z e n t}=H Z V \cdot m t t_{f-z e n t}-T V_{k-p r a ̈} \\
& m t t_{f-z e n t}=\text { mittlere Transitzeit von ICG zwischen A. pulmonalis und Aorta aus Gleichung 2-11 } \\
& T V_{k-p r a ̈ ~}=\text { Präpulmonales ,thermoakzessibles“ Verteilungsvolumen der Kälte }
\end{aligned}
$$

\section{5 „Extravaskuläres Lungenwasser““}

Das „extravaskuläre Lungenwasser“ $(E V L W)$ entspricht der Differenz aus dem Verteilungsraum des Indikators Kälte, der dem gesamten intra- und extravasalen Raum des kleinen Kreislaufs entspricht (ZTV, Gleichung 2-10), und dem Verteilungsraum des Indikators Indocyaningrün $\left(V_{d-z e n t}\right.$, Gleichung 2-12) und ergibt sich zu:

\section{Gleichung 2-13}

$$
\begin{aligned}
E V L W & =Z T V-V_{d-z e n t} \\
& =H Z V \cdot m t t_{k-z e n t}^{\prime}-\left(H Z V \cdot m t t_{f-z e n t}-T V_{k-p r a ̈}\right) \\
& =H Z V \cdot\left(m t t_{k-z e n t}^{\prime}-m t t_{f-z e n t}\right)+T V_{k-p r a ̈}
\end{aligned}
$$




\section{6 ,In vivo“-Bestimmung des, ,zirkulierenden“ Blutvolumens}

Unter Verwendung etwas aufwendigerer pharmakokinetischer Modelle (Cutler 1979; Hoeft et al. 1994) ist es möglich geworden, den gesamten Informationsgehalt einer Indikatorverdünnungskurve zu nutzen. Dementsprechend kann mit Hilfe der stochastischen Systemanalyse aus der Rezirkulationskinetik des ICGs eine sogenannte Kreislauf-Transportfunktion $g_{f}(t)$ berechnet werden, die sowohl die Verteilungs- als auch die Eliminationsphase des Farbstoffs beschreibt und dadurch zusätzliche Parameter zur Beschreibung des Flüssigkeitsstatus des Patienten ermittelt.

\subsubsection{Die Kreislauf-Transportfunktion und der rekursive Faltungsalgorithmus}

Nach zentralvenöser Bolusapplikation des Indikators $I C G$ in den rechten Vorhof läßt sich am Meßort in der absteigenden Aorta eine typische Farbstoffverdünnungskurve $\left(c_{f-a o}(t)\right.$, s. Abbildung 2-4) registrieren. Dieser Konzentrations-Zeitverlauf des Indikators stellt die Summe der Konzentrations-Zeitsignale des durch Dispersionsmechanismen während der Lungenpassage transformierten initialen Farbstoff-Bolus (,first pass“", $c_{f f p}(t)$ ) und des rezirkulierenden Anteils des Indikators $\left(c_{f-r e z}(t)\right)$ dar, der nach Passage eines Körperkompartimentes und erneutem Lungendurchfluß wiederum am Meßort erscheint (Abbildung 2-4):

\section{Gleichung 2-14}

$$
c_{f-a o}(t)=c_{f-f p}(t)+c_{f-r e z}(t)
$$

Mit Hilfe der stochastischen Systemanalyse kann der Transportprozeß des Indikators ICG durch den Körperkreislauf durch ein Faltungsintegral beschrieben werden (vgl. Abschnitt 2.1.3.2). Als Eingangsfunktion dient hier die aortale Farbstoffverdünnungskurve $c_{f-a o}(t)$, die durch Faltung mit einer Transportfunktion $g_{f}(t)$, die die spezifischen Dispersionsmechanismen des Indikators während seiner Kreislaufpassage beschreibt, den Konzentrations-Zeitverlauf des rezirkulierenden Indikatoranteils $\left(c_{f-r e z}(t)\right)$ als Ausgangsfunktion ermittelt. Unter Berücksichtigung des Indikatorverlustes im Sinne einer konzentrationsabhängigen Elimination des ICGs (vgl. Abschnitt 2.1.3.1) durch die Leber, rezirkuliert nur ein Anteil $R$ des Farbstoffs und die Ausgangsfunktion ergibt sich zu:

\section{Gleichung 2-15}

$$
\begin{array}{ll}
c_{f-r e z} & (t)=R \cdot \int_{0}^{t} g_{f}(t-u) c_{f-a o}(u) d u \\
c_{f-r e z}(t) & =\text { Konzentrations-Zeitverlauf des rezirkulierenden Farbstoffes } \\
c_{f-a o}(t) & =\text { Konzentrations-Zeitverlauf von } I C G \text { in der Aorta } \\
g_{f}(t) & =\text { Kreislauf-Transportfunktion für } I C G \\
u & =\text { Integrationsvariable der Zeit } \\
R & =\text { Rezirkulationsfraktion, wobei } 0<R=(1-E)<1 ; E \text { entspricht der Eliminationsfraktion }
\end{array}
$$


Durch Substitution von $c_{f-r e z}$ in die Gleichung 2-14 resultiert die $c_{f-a o}$ als:

Gleichung 2-16

$$
c_{f-a o}(t)=c_{f-f p}(t)+R \cdot \int_{0}^{t} g_{f}(t-u) c_{f-a o}(u) d u
$$

Anhand des Zusammenhangs in Gleichung 2-16 kann somit $c_{f-a o}(t)$ durch einen sogenannten rekursiven Faltungsalgorithmus, der die Verteilung, Rezirkulation und Elimination des Indikators simuliert, beschrieben werden (Cutler 1979; van Rossum et al. 1989; Hoeft et al. 1994). Die Ausgangsfunktion dieser Faltung, die das Ergebnis des spezifischen Transportprozesses durch den Körperkreislauf darstellt, erscheint wieder als Eingangsfunktion (s. Abbildung 2-4).
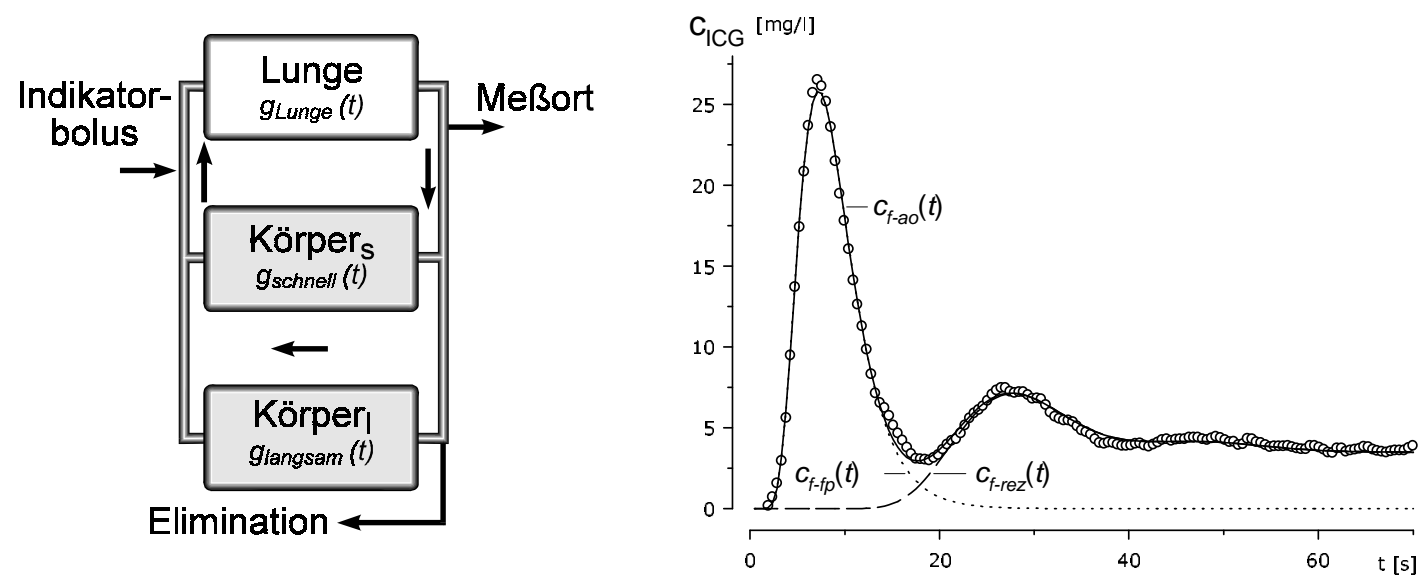

Abbildung 2-4. Mathematisches Rezirkulationsmodell und aortale Farbstoffverdünnungskurve

Nach zentralvenöser, bolusförmiger ICG-Applikation in den rechten Vorhof läßt sich am Meßort eine typische Farbstoffverdünnungskurve als Summe der Konzentrations-Zeitsignale des initialen Farbstoff-Bolus (,first pass") nach Lungenpassage $\left(c_{f-f p}(t)\right)$ und des rezirkulierenden Indikatoranteils $\left(c_{f-r e z}(t)\right)$ registrieren. Unter Verwendung eines mathematischen Rezirkulationsmodells und Zugrundelegens einer zweikompartimentellen Kreislaufanalyse läßt sich der Transportprozeß des Indikators während seiner Kreislaufpassage durch ein Faltungsintegral, bestehend aus einer Transportfunktion und einem Eingangssignal in das System, beschreiben. Das Ausgangssignal, das den Konzentrations-Zeitverlauf des rezirkulierenden Indikatoranteils darstellt, resultiert entsprechend aus der Faltung des aortalen Eingangssignals $c_{f-a o}(t)$ mit der Transportfunktion $g_{f}(t)$.

In der Abbildung Darstellung der Meßdaten $\left(^{\circ}\right)$, der Primärkurve (.....), der Indikatorrezirkulation (- - ) und der angepaßten Modellfunktion (-).

$g_{\text {Lunge }}(t)$ : Transportfunktion der Lunge; $g_{\text {schnell }}(t)$ : Transportfunktion des schnellen Kreislaufkompartimentes; $g_{\text {langsam }}(t)$ : Transportfunktion des langsamen Kreislaufkompartimentes.

Durch ein nichtlineares Verfahren auf der Basis der kleinsten Abstandsquadrate (Deuflhard und Apostolescu 1980) erfolgte die Abgrenzung der Primärkurve $c_{f-f p}(t)$ von dem rezirkulierenden Anteil $c_{f-\text {-rez }}(t)$ des Indikators, indem es an die aortal registrierte $I C G$-Verdünnungskurve vom Beginn des Konzentrationsanstiegs bis zu $33 \%$ des Maximums auf dem abfallenden 
Kurvenanteil eine Modellfunktion angepaßt wurde. Eine sehr gute Beschreibung des „first pass“ zeigte sich durch die Anpassung einer Lognormal-Verteilung als Modellfunktion.

\subsubsection{Das zweikompartimentelle Modell für die Kreislauf-Transportfunktion}

Nach Abgrenzung des „first pass“ (Primärkurve) durch die Anpassung der Lognormal-Verteilung (s. Abbildung 2-4), wurde das abgetrennte Konzentrations-Zeitsignal des rezirkulierenden Indikators mit Hilfe des rekursiven Faltungsalgorithmus gemäß Gleichung 2-16 simuliert, wobei die an die Primärkurve angepaßte Funktion $c_{f-f p}^{\prime}(t)$ als Eingangsfunktion für den Faltungsalgorithmus diente.

Durch eine Entfaltungsprozedur zur Lösung des inversen Problems von Gleichung 2-16 und Ermittlung der Transportfunktion ( $s$. Abschnitt 2.1.3.3) wurden initiale Schätzwerte der Parameter einer für die Kreislauf-Transportfunktion geeigneten Modellfunktion sowie der Faktor $R$ solange iterativ verändert, bis eine optimale Übereinstimmung zwischen der durch Faltung des Eingangssignals $c_{f-a o}(t)$ mit dieser Modellfunktion und Addition der $c_{f-f p}^{\prime}(t)$ mathematisch simulierten Indikatorrezirkulationskinetik $c_{f-a o}(t)$ und den gemessenen Konzentrations-Zeitverlauf erreicht wurde.

Sehr gute Übereinstimmungen zwischen berechneten und gemessenen Werten wurden jedoch erst nach Zugrundelegen eines zweikompartimentellen Modells (Henthorn et al. 1992; Caldini et al. 1974; Hoeft et al. 1994) für die Kreislauf-Transportfunktion $g_{f}(t)$ erreicht, die sich als Summe von zwei linksschiefen Lognormal-Verteilungen $g_{1,2}(t)$ darstellte ( $s$. Abbildung 2-4 und Gleichung 2-18). Jeder Versuch die gesuchte Kreislauf-Transportfunktion mit einer einzelnen Modellfunktion zu simulieren, führte zu systematischen Abweichungen. Dementsprechend gilt:

\section{Gleichung 2-17}

$$
\begin{aligned}
& R \cdot g(t)=R_{1} \cdot g_{1}(t)+R_{2} \cdot g_{2}(t) \\
& R_{1}, R_{2}=\text { Gewichtungsfaktoren der beiden Körperkompartimente mit } R_{1}+R_{2} \leq 1
\end{aligned}
$$

Definitionsgemäß beträgt die Fläche unter den Transportfunktionen 1 und demzufolge können $R_{I}$ und $R_{2}$ als Gewichtungsfaktoren der beiden Körperkompartimente angesehen werden, wobei sich ein schnelles und ein langsames Kompartiment diskriminieren läßt. Henthorn et al. (1992) beschreiben, daß das schnelle Kompartiment die Perfusion der in einem Dauerfunktions-Zustand befindenden Organe, wie beispielsweise Herz, Nieren oder Gehirn, das langsame Kompartiment vorwiegend den intestinalen Blutfluß repräsentiert (Abbildung 2-5). 


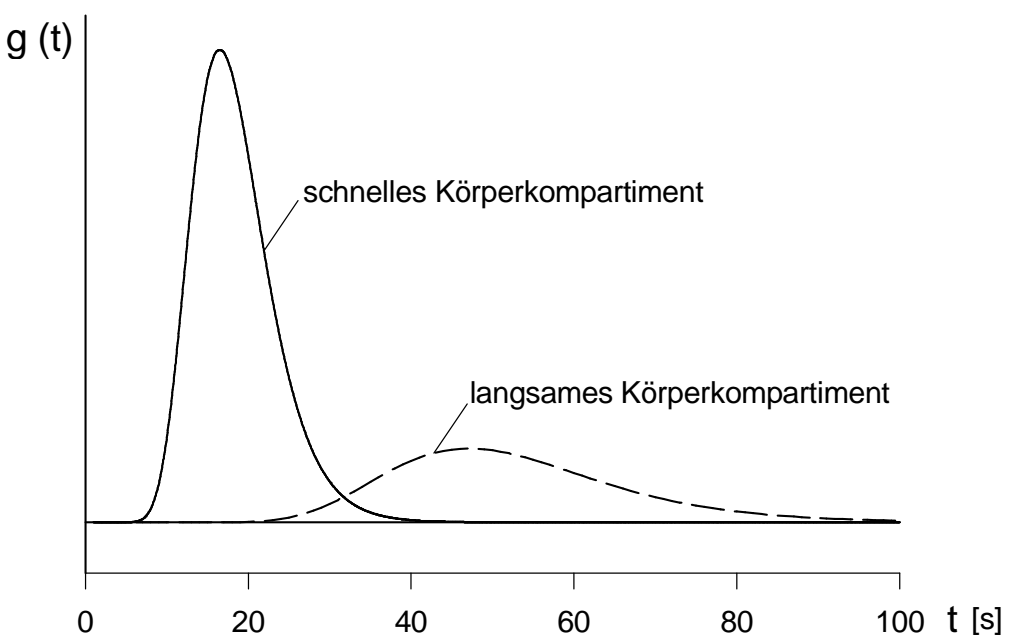

Abbildung 2-5. Kreislauf-Transportfunktion: $g_{f}(t)=g_{l}(t)+g_{2}(t)$

Beispiel einer Kreislauf-Transportfunktion, als Summe von zwei Lognormal-Verteilungen, die das schnelle $\left(g_{l}(t)\right)$ und das langsame $\left(g_{2}(t)\right)$ Körperkompartiment charakterisieren.

Die Transportfunktionen der beiden Körperkompartimente errechnen sich aus:

Gleichung 2-18

$$
\begin{aligned}
& g_{1,2}\left(t, \mu_{1,2}, \sigma_{1,2}\right)=\frac{1}{\sigma_{1,2} \cdot t \cdot \sqrt{2 \pi}} \cdot \exp \left(-\frac{\left(\ln t-\mu_{1,2}\right)^{2}}{2 \sigma_{1,2}{ }^{2}}\right) \\
& \mu_{1,2}=\text { Erwartungswert der Lognormal-Verteilung } \\
& \sigma_{1,2}=\text { Standardabweichung der Lognormal-Verteilung }
\end{aligned}
$$

Durch Substitution von $m t t_{1,2}=\exp \left(\mu_{1,2}+\frac{{\sigma_{1,2}}^{2}}{2}\right)$ in Gleichung 2-18 ergibt sich:

\section{Gleichung 2-19}

$$
g_{1,2}\left(t, m t t_{1,2}, \sigma_{1,2}\right)=\frac{1}{\sigma_{1,2} \cdot t \cdot \sqrt{2 \pi}} \cdot \exp \left(-\frac{\ln \left(\frac{t}{m t t_{1,2}}\right)+\frac{\sigma_{1,2}^{2}}{2}}{2 \sigma_{1,2}{ }^{2}}\right)
$$

\subsubsection{Verteilungsvolumina auf der Basis des mathematischen Rezirkulationsmodells}

Gemäß Gleichung 2-3 berechnet sich die mittlere Kreislauf-Transitzeit für ICG $\left(m t_{f-z i r k}\right)$ aus den gewichteten mittleren Transitzeiten $m t t_{f 1}$ und $m t t_{f 2}$ der Einzeltransportfunktionen $g_{l}(t)$ und $g_{2}(t)$ : 
Gleichung 2-20

$$
m t t_{f-z i r k}=\frac{R_{1} \cdot m t t_{f_{1}}+R_{2} \cdot m t t_{f_{2}}}{R_{1}+R_{2}}
$$

Unter Berücksichtigung der Gewichtung jedes Flüssigkeitskompartimentes ergeben sich entsprechend die Verteilungsräume der Einzelkompartimente, deren Summe das ,zirkulierende“ Blutvolumen $V_{d-z i r k}$ repräsentiert, gemäß Gleichung 2-4 zu:

\section{Gleichung 2-21}

$$
\begin{aligned}
V_{d-z i r k} & =V_{d-z i r k_{1}}+V_{d-z i r k_{2}} \\
& =H Z V \cdot \frac{R_{1}}{R_{1}+R_{2}} \cdot m t t_{f_{1}}+H Z V \cdot \frac{R_{2}}{R_{1}+R_{2}} \cdot m t t_{f_{2}} \\
& =H Z V \cdot m t t_{f-z i r k}
\end{aligned}
$$

Die beiden Kompartimente werden mit entsprechenden Anteilen des Herzzeitvolumens versorgt, die jeweils betragen:

\section{Gleichung 2-22}

$$
H Z V_{1,2}=\frac{R_{1,2}}{R_{1}+R_{2}} \cdot H Z V
$$




\section{Material und Methoden}

\subsection{Patienten}

Nach Prüfung und Zustimmung des Studienprotokolls durch die hiesige Ethikkomission wurden die Untersuchungen der vorliegenden Studie an 20 erwachsenen Patienten beiderlei Geschlechts, die sich in der Klinik für Thorax-, Herz- und Gefäßchirurgie der Universität Göttingen elektiv einer koronarchirurgischer Operation unter extrakorporaler Zirkulation unterziehen mußten, durchgeführt.

Nach ausführlicher Aufklärung über die klinische und wissenschaftliche Bedeutung sowie die möglichen Risiken der zusätzlichen Untersuchungen erteilten die Patienten am Vorabend der Operation ihr Einverständnis, an der Studie teilzunehmen. Bei allen Patienten wurde eine Myokardrevaskularisation aufgrund einer bestehenden und angiographisch gesicherten koronaren Herzkrankheit $(K H K)$ vorgenommen. Unter doppelblind-randomisierten Bedingungen erfolgte die Zuteilung der Patienten in zwei Gruppen. Daraufhin erhielten die Patienten der ersten Gruppe kein Glucocorticoid (PLC-, Placebo-Gruppe) und die der zweiten Gruppe $1 \mathrm{mg} \cdot \mathrm{kg}^{-1}$ Dexamethason (DXM-, Dexamethason-Gruppe) intravenös nach Narkoseeinleitung.

Für eine Teilnahme an den Untersuchungen wurden Ausschlußkriterien festgesetzt. Es wurden nur Patienten, die keine der folgenden Kriterien aufwiesen, in die Studie aufgenommen:

- Lebensalter größer als 75 Jahre

- Linksventrikuläre Ejektionsfraktion kleiner als $50 \%$

- Instabile Angina pectoris oder signifikante Hauptstammstenose

- Anamnestischer Hinweis auf Allergie und besonders Jodallergie

- Periphere arterielle Verschlußkrankheit mit Beteiligung der Femoralgefäße

- Pathologische Nierenretentionswerte (Kreatinin, Harnsäure)

- Pathologische Leberenzymwerte ( $\gamma$-GT, GPT, GOT) oder erhöhtes Serumbilirubin

- Präoperativ diagnostiziertes Herzklappenvitium

\subsection{Ablauf der Untersuchungen}

Vor Beginn der Studie wurde ein Protokoll erstellt, in dem der organisatorische und zeitliche Ablauf der hämodynamischen und laborchemischen Untersuchungen festgelegt wurde. Die Messungen wurden in einem Untersuchungszeitraum zwischen Narkoseeinleitung und zwanzig Stunden nach Operationsende durchgeführt. Gemäß dem im Protokoll festgelegten Zeitplan erfolgte die Bestimmung der Laborparameter sowie die Registrierung der Indikatordilutionskurven und der korrespondierenden hämodynamischen Parameter unmittelbar nach Einleitung der Narkose (n.N.) und sowie (1h), sechs (6h) und zwanzig (20h) Stunden nach Beendigung der Operation. Die Auswertung der während des Untersuchungszeitraums 
registrierten Daten erfolgte zu einem späteren Zeitpunkt, so daß die Kenntnis von den zusätzlich berechneten Parametern keinen Einfluß auf die individuelle therapeutische Intervention hatte.

Die Patienten wurden perioperativ durch Anästhesisten und Intensivmediziner betreut. Die Gruppenzugehörigkeit der Patienten wurde den behandelnden Ärzten nicht mitgeteilt, damit eine bedarfsorientierte und von der Studie unabhängige Therapie gewährleistet wurde. Um die durch unterschiedliche Behandlungsmöglichkeiten eingebrachte Variation möglichst gering zu halten und die Behandlung perioperativ in einem vertretbaren Rahmen zu standardisieren, wurden folgende therapeutische Rahmenanordnungen in das Studienprotokoll aufgenommen:

- Während der ersten 20 Stunden postoperativ wurde der Bassisflüssigkeitsbedarf durch Dauerinfusion von $40 \mathrm{ml} \cdot \mathrm{kg}^{-1} \mathrm{kristalloider} \mathrm{Lösung} \mathrm{gedeckt}$

- Bei Abfall des Hämoglobinwertes unter $8 \mathrm{~g} \cdot \mathrm{dl}^{-1}$ erfolgte die zusätzliche Gabe von Erythrozyten-Konzentraten

- Bei Gesamteiweißgehalt von weniger als $4 \mathrm{~g} \cdot \mathrm{dl}^{-1}$ wurden proteinhaltige Lösungen substituiert

- Bei Absinken von dem systemischen Blutdruck oder der Füllungsdrücke zunächst wurde die Infusion kristalloider Lösungen als rasche Substitution von 200-300 ml innerhalb weniger Minuten forciert

- Bei fehlendem Ansprechen Infusion kolloider Lösungen

- Bei verstärkter Blutungsneigung aufgrund von Gerinnungsstörungen erfolgte die Transfusion von Frischplasma (FFP) und gegebenenfalls von Thrombozytenkonzentraten

- Innerhalb des ersten postoperativen Tages wurde eine Diurese von 1-2 $\mathrm{ml} \cdot \mathrm{kg}^{-1} \cdot \mathrm{h}^{-1}$ und eine negative Gesamtbilanz von mindestens $1500 \mathrm{ml}$ angestrebt

\subsubsection{Kathetherisierung der Patienten und Narkoseeinleitung}

Die Patienten wurden am Vorabend des Eingriffs und unmittelbar vor Transport in den Operationssaal mit einer oralen Gabe von $0,03 \mathrm{mg} \cdot \mathrm{kg}^{-1}$ Körpergewicht Flunitrazepam ${ }^{4}$ (Maximaldosis $2 \mathrm{mg}$ ) prämediziert und erhielten bis zum Operationstag ihre herzwirksame Vormedikation in gewohnter Dosierung. Nach Ankunft der Patienten im Einleitungsraum und unter kontinuierlicher Überwachung der Vitalfunktionen (s. Abschnitt 3.4.2) wurde in örtlicher Betäubung ein zentraler Venenkatheter ${ }^{5}$ und eine $8,5 \mathrm{~F}-V_{\text {Ventil-Schleuse }}{ }^{6}$ in die rechte Vena jugularis interna eingeführt sowie in Seldinger-Technik eine 5F-Schleuse ${ }^{7}$ mit Seitenweg zur arteriellen Blutdruckmessung in die linke Arteria femoralis.

\footnotetext{
${ }^{4}$ Rohypnol ${ }^{\circledR}$, Hoffmann-LaRoche AG, Grenzach-Wyhlen, BRD

${ }^{5}$ Arrows International Inc., Reading/PA, USA

${ }^{6}$ Arrows International Inc., Reading/PA, USA

${ }^{7}$ Pulsion ${ }^{\circledR}$ 5F Einführungs-Set, Pulsion Medical Systems, München, BRD
} 
Nach ausreichender Präoxygenierung wurde die Narkose mit $2 \mu \mathrm{g} \cdot \mathrm{kg}^{-1}$ Sufentanil $^{8}$ und $0,1 \mathrm{mg} \cdot \mathrm{kg}^{-1} \quad$ Körpergewicht Pancuroniumbromid ${ }^{9}$ eingeleitet. Anschließend erfolgte die nasotracheale Intubation und eine kontrollierte normocarbische Beatmung ${ }^{10}$ bei einem $\mathrm{FiO}_{2}$ von 0,5. Angestrebt wurde ein von einem handelsüblichen Capnometer ${ }^{11}$ erfaßtes $\mathrm{CO}_{2}$ zwischen 4,5 und $5 \mathrm{Vol} \%$ und eine pulsoximetrisch gemessene arterielle Sauerstoffsättigung von mehr als $95 \%$. Durch kontinuierliche Infusion von $1-1,5 \mu \mathrm{g} \cdot \mathrm{kg}^{-1} \cdot \mathrm{h}^{1}$ Sufentanil und $45-90 \mu \mathrm{g} \cdot \mathrm{kg}^{-1} \cdot \mathrm{h}^{1}$ Midazolam $^{12}$ sowie gegebenenfalls zusätzliche Einzelgaben wurde eine angemessene Narkosetiefe aufrechterhalten.

Nach Narkoseeinleitung wurde über die bereits gelegte 8,5F-Schleuse in der rechten Vena jugularis interna ein 7F-Swan-Ganz-Thermodilutions-Katheter ${ }^{13}$ in eine der Arteriae pulmonales eingeschwemmt. Anschließend wurde über die Schleuse in der linken A. femoralis ein kombinierter 4F-Fiberoptik-Thermistor-Katheter ${ }^{14}$ in die thorakale Aorta descendens mit seiner Spitze etwas weiter als die Zwerchfellhöhe vorgeschoben. Seine Lage wurde solange korrigiert, bis das optische Signal nach Anschluß des Katheters am Registriergerät ${ }^{15}$ eine ausreichende Pulsatilität zeigte.

Unter doppelblind placebo-kontrollierten Bedingungen erfolgte nun die Randomisierung der Patienten in die zwei Untersuchungsgruppen, wobei den Patienten der Kontrollgruppe $10 \mathrm{ml}$ $0,9 \%$ Kochsalzlösung, denen der Verumgruppe $1 \mathrm{mg} \cdot \mathrm{kg}^{-1}$ Körpergewicht Dexamethason ${ }^{16}$ aufgezogen auf einer $10 \mathrm{ml}$-Spritze mit physiologischer Kochsalzlösung appliziert wurde.

\subsubsection{Operationsablauf unter Einsatz der extrakorporalen Zirkulation}

Nach medianer Sternotomie wurde in den meisten Fällen zunächst die linke Arteria thoracica interna zwischen der unteren Thoraxapertur und der Pleurakuppel dargestellt und freipräpariert. Simultan dazu wurde ein Venensegment der distalen Vena saphena magna entnommen.

Nach Längseröffnung des Perikards und systemischer Gabe von $300 \mathrm{IE} \cdot \mathrm{kg}^{-1} \mathrm{Heparin}^{17}$ wurde über eine Kanüle in der Aorta ascendens und getrennter Kanülierung der oberen und unteren Hohlvene die Herz-Lungen-Maschine angeschlossen und die extrakorporale Zirkulation eingeleitet. Bei milder Ganzkörperhypothermie von ca. $30{ }^{\circ} \mathrm{C}$ und externer lokaler Kühlung des Herzens durch Eiswasser wurde nach Abklemmen der Aorta ascendens ein pharmakologischer Stillstand mittels

\footnotetext{
${ }^{8}$ Sufenta $^{\circledR}$, Janssen-Cilag GmbH, Neuss, BRD

${ }^{9}$ Pancuronium ${ }^{\circledR}$, Organon Teknika Medizinische Produkte GmbH, Eppelheim, BRD

${ }^{10} \mathrm{CATO}^{\circledR}$ Ventilator, Dräger Medizintechnik GmbH, BRD

${ }^{11}$ Datex Normocap ${ }^{\circledR}$, Datex, Helsinki, Finnland

${ }^{12}$ Dormicum $^{\circledR}$, Hoffmann-LaRoche AG, Grenzach-Wyhlen

${ }^{13}$ Arrows International Inc., Reading/PA, USA

${ }^{14}$ PV 2022, Pulsion Medical Systems, München, BRD

${ }^{15}$ Pulsion COLD Z-02 ${ }^{\circledR}$, Pulsion Medical Systems, München, BRD

${ }^{16}$ Fortecortin $^{\circledR}$, Merck, Darmstadt, BRD

${ }^{17}$ Liquemin $^{\circledR}$, Hoffmann-LaRoche AG, Grenzach-Wyhlen, Schweiz
} 
Infusion von $30 \mathrm{ml} \cdot \mathrm{kg}^{-1}$ eisgekühlter Bretschneider-Kardioplegielösung ${ }^{18}$ in den Aortenbulbus induziert. Das abgeleitete Perfusat wurde über den rechten Vorhof verworfen.

Die extrakorporale Zirkulation während der Operation erfolgte als totaler kardiopulmonaler Bypass unter Verwendung von Membran-Oxygenatoren zum Gasaustausch ${ }^{19}$ und angetrieben mit einer Zentrifugalpumpe ${ }^{20}$. Zum Schutz vor Mikroembolisationen diente ein in der arteriellen Linie des extrakorporalen Kreislaufs eingebauter Filter ${ }^{21}$. Die Vorfüllung der HLM betrug $2000 \mathrm{ml}$ und setzte sich aus $1150 \mathrm{ml}$ Ringer-Laktatlösung, $250 \mathrm{ml}$ Glucose $5 \%, 500 \mathrm{ml}$ Haes $6 \%$ und $100 \mathrm{ml} \mathrm{Na-Bikarbonat} \mathrm{zusammen.}$

Nach Wiederaufwärmung auf mindestens $35{ }^{\circ} \mathrm{C}$ und entsprechender Reperfusion wurde bei stabilen Kreislaufverhältnissen die EKZ ausgeleitet. Anschließend erfolgte die Dekanülierung zunächst der Hohlvenen und nach Antagonisierung der Heparinwirkung mit Protaminsulfat ${ }^{22}$ der Aorta ascendens.

Das während der Operation anfallende Blut wurde entweder in das Reservoir der HLM geleitet oder in einem Cell-saver ${ }^{23}$ aufgefangen. Nach entsprechender Aufbereitung im Cell-saver (vgl. Abschnitt 3.3) wurde das Blut erneut transfundiert.

\subsection{Bilanzierung}

$\mathrm{Zu}$ jedem Meßzeitpunkt wurde eine Ein- und Ausfuhrbilanz im Sinne eines Gewinnes oder Verlustes in $m l$ kristalloider Lösung, kolloider Lösung (entsprechend einem Proteingehalt von $5 \mathrm{~g} \%)$ sowie Erythrozytenvolumen erstellt. Um den direkten Einfluß der Operation und der extrakorporalen Zirkulation beurteilen zu können, erfolgte zusätzlich eine Flüssigkeitsbilanzierung zum Ende der Operation $(O p E)$.

In der Einfuhrbilanz wurden berücksichtigt:

- Das primäre Füllvolumen der HLM, das komplett in die kristalloide bzw. kolloide Einfuhrbilanz einging

- Die Erythrozytenkonzentrate, die entsprechend einem Erythrozytenvolumen von $60 \%$, einem Kolloidvolumen von $39 \%$ und einem Wasseranteil von $1 \%$ bilanziert wurden ${ }^{24}$

- Die Autotransfusion des während der Operation unter Verwendung des „Cell-saver“ aufgefangenen Blutes. Der Mittelwert der vor, nach und in 20-minütigen Abständen während der EKZ bestimmten Hämatokritwerte wurde als Hämatokritwert des aus dem Patienten oder der HLM im Cell-saver-Reservoir gesammelten Blutes angenommen. Zur Aufbereitung wurde

\footnotetext{
${ }^{18}$ Custodiol $^{\circledR}$, Dr. F. Köhler Chemie GmbH, Alsbach/Bergstraße, BRD

${ }^{19}$ Maxima Hollow Fiber Oxygenator, Johnson \& Johnson Cardiovascular, Anaheim/CA, USA

${ }^{20}$ Bio-Medicus Bio-Pump ${ }^{\circledR}$, Medtronic Inc., Eden Prairie/MN, USA

${ }^{21}$ Sartorius AG, Göttingen, BRD

${ }^{22}$ Protamin $^{\circledR}$, Hoffmann-LaRoche AG, Grenzach-Wyhlen, BRD

${ }^{23}$ Shiley ${ }^{\mathrm{TM}}$ Therapeutic Autotransfusion System, Dideco, Mirandola, Italien

${ }^{24}$ nach Angaben der Blutbank des Universitätsklinikums in Göttingen
} 
das Cell-saver-Blut zentrifugiert und gleichzeitig mit isotoner Kochsalzlösung gewaschen. Dies hatte allerdings zur Folge, daß bei ausgeglichener Erythrozytenbilanz der Plasmaanteil des im Cell-saver-Reservoir aufgefangenen Blutes verloren ging und dementsprechend der kolloidalen Ausfuhr zuzuordnen ist. Dagegen wurde ein Volumenanteil des aufbereiteten Blutes von ca. $58 \%$ unter Berücksichtigung eines Erythrozytenvolumens ${ }^{25}$ von $42 \%$ als kristalloide Lösung zugeführt und in die kristalloide Bilanz eingerechnet.

Die Ausfuhrbilanz beinhaltete:

- Das am Ende der Operation in der HLM verbleibende Volumen sowie postoperative Verluste über die Drainagen, die in die Ausfuhrbilanz für Erythrozyten und Kolloide eingingen.

- Ein zusätzlicher intraoperativer Blutverlust (Tücher, Kompressen) von ca. $400 \mathrm{ml}$

- Wasserverluste bedingt durch die Diurese, den Verlust über die Magensonde und die perspiratio insensibilis. Letztere wurde während der Operationsdauer sowie unter Spontanatmung mit $25 \mathrm{ml} \cdot \mathrm{h}^{-1}$ und für die Zeit unter der maschinellen Beatmung mit $12,5 \mathrm{ml} \cdot \mathrm{h}^{-1}$ in die kristalloide Ausfuhrbilanz eingerechnet.

\subsection{Meßmethoden}

\subsubsection{Laborchemische Untersuchungen}

Das Studienprotokoll sah zu jedem Meßzeitpunkt Blutabnahmen zur Bestimmung von üblichen laborchemischen Parametern vor. In mit Heparin ${ }^{26}$ benetzten Spritzen wurden vor Durchführung der Messungen $2 \mathrm{ml}$ arterielles aus der Femoralis-Schleuse und $3 \mathrm{ml}$ gemischtvenöses Blut aus dem distalen Lumen des Swan-Ganz-Katheters abgenommen. Aus den beiden Blutproben wurde die Sauerstoffsättigung $\left(\mathrm{SO}_{2}\right)$ und der Hämoglobingehalt $(\mathrm{Hb})$ ermittelt ${ }^{27}$ und eine Blutgasanalyse zur Bestimmung des Sauerstoff- $\left(\mathrm{pO}_{2}\right)$ und Kohlendioxidpartialdruckes $\left(\mathrm{pCO}_{2}\right)$ durchgeführt ${ }^{28}$. Die Analyse ergab zusätzlich Daten zum Wasser- und Elektrolythaushalt $\left(\mathrm{K}^{+}-\mathrm{Na}^{+}-\right.$ Konzentration) sowie zum Säure-Basen-Haushalt ( $p H$, Standardbikarbonat, Base-Excess). Die Bestimmung des Hämatokrits $(H k t)^{29}$ erfolgte aus der arteriellen Probe. Nach anschließendem Zentrifugieren der venösen Probe über zehn Minuten bei $4.000 \mathrm{U} \cdot \mathrm{min}^{-1}$ wurde aus dem Serum die Glucose- $(G l c)^{30}$ und Totalprotein-Konzentration $(T P)$ ermittelt. Zusätzlich wurden aus $2 \mathrm{ml}$ gemischtvenösem EDTA-Blut ${ }^{31}$ wurden die Leukozyten- (WBC) und Thrombozytenzahl (PLT) gemessen.

\footnotetext{
${ }^{25}$ Die Untersuchung von zehn aufbereiteten Autotransfusions-Konserven erbrachte einen konstanten mittleren zellulären Anteil von ca. $42 \%$.

${ }^{26}$ Liquemin $^{\circledR}$, Hoffmann-LaRoche AG, Grenzach-Wyhlen, Schweiz

${ }^{27}$ OSM3-Hemoximeter, Radiometer, Copenhagen, Dänemark

${ }^{28}$ ABL Radiometer, Copenhagen, Dänemark

${ }^{29}$ Haemofuge A, Heraeus Holding GmbH, Hannover, BRD

${ }^{30}$ Test-Combination Best.-Nr. 124281, Boehringer Mannheim GmbH Diagnostica, Mannheim, BRD

${ }^{31}$ Sarstedt Aktiengesellschaft \& Co, Nümbrecht, BRD
} 


\subsubsection{Hämodynamische Kreislaufparameter}

Die Herzfrequenz $(H f)$ und die intravasalen Drücke des systemischen und kleinen Kreislaufs wurden vom Monitoring-System ${ }^{32}$ ermittelt. Zur Bestimmung der $H f$ diente als Quelle die II. Extremitätenableitung des Oberflächen-EKG's. Über den Seitenzweig der 5F-FemoralisSchleuse wurde der systemarterielle systolische, diastolische und Mitteldruck (APs, APd, MAP) gemessen. Die Registrierung des Zentralvenendruckes (ZVD) erfolgte über das proximale Lumen, der pulmonalarteriellen Drücke ( $P A P s, \quad P A P d, \quad M P A P)$ sowie des pulmonalkapillären Verschlußdruckes $(P C W P)$ über das distale Lumen des Pulmonalis-Katheters.

Die Körpertemperatur der Patienten wurde kontinuierlich mittels rektaler Temperatur-Sonde ${ }^{33}$ überwacht. Während der Messungen wurde die Bluttemperatur $\left(T_{a o}\right)$, gemessen über den in der Aorta descendens plazierten Fiberoptik-Katheter, ebenso registriert.

Nach Standardformeln konnten bei jedem Meßzeitpunkt zusätzlich zu den direkt gemessenen Werten folgende Kreislaufparameter berechnet werden.

\section{Herzzeitvolumen-Index $(C I)$ :}

$$
\begin{aligned}
& C I=\frac{H Z V}{K O F} \quad\left[1 \cdot \mathrm{min}^{-1} \cdot \mathrm{m}^{-2}\right] \\
& H Z V=\text { Herzzeitvolumen }\left[1 \cdot \mathrm{min}^{-1}\right] \\
& K O F=\text { Körperoberfläche }\left[\mathrm{m}^{2}\right]
\end{aligned}
$$

\section{Schlagvolumen-Index $(S V I)$ :}

$$
\begin{array}{ll}
S V I= & \frac{C I}{H f} \\
C I & =\text { Herzzeitvolumen-Index }\left[1 \cdot \mathrm{min}^{-1} \cdot \mathrm{m}^{-2}\right] \\
H f & =\text { Herzfrequenz }\left[\mathrm{min}^{-1}\right]
\end{array}
$$

\section{Systemischer Gefäßwiderstands-Index (SVRI):}

$$
\begin{aligned}
S V R I & =\frac{(M A P-Z V D) \cdot 79,9}{C I} \\
M A P & =\text { systemarterieller Mitteldruck }[\mathrm{mmHg}] \\
Z V D & =\text { zentralvenöser Druck }[\mathrm{mmHg}] \\
C I & =\text { Herzzeitvolumen-Index }\left[1 \cdot \mathrm{min}^{-1} \cdot \mathrm{m}^{-2}\right]
\end{aligned}
$$$$
\left[\text { dyn } \cdot \mathrm{sec} \cdot \mathrm{m}^{2} \cdot \mathrm{cm}^{-5}\right. \text { ] }
$$

\footnotetext{
${ }^{32}$ Sirecust 1281, Siemens Medizintechnik AG, Erlangen, BRD oder HP CMS-Patientenmonitor, Hewlett Packard, Bad Homburg, BRD

${ }^{33}$ Servomed SM 108, Hellige, BRD
} 
Pulmonaler Gefäßwiderstands-Index (PVRI):

$$
\begin{aligned}
P V R I & =\frac{(M P A P-P C W P) \cdot 79,9}{C I} \quad\left[\mathrm{dyn} \cdot \mathrm{sec} \cdot \mathrm{m}^{2} \cdot \mathrm{cm}^{-5}\right] \\
M P A P & =\text { pulmonalarterieller Mitteldruck }[\mathrm{mmHg}] \\
P C W P & =\text { pulmonalkapillärer Verschlußdruck }[\mathrm{mmHg}] \\
C I & =\text { Herzzeitvolumen-Index }\left[1 \cdot \mathrm{min}^{-1} \cdot \mathrm{m}^{-2}\right]
\end{aligned}
$$

\subsubsection{Doppelindikatorverdünnung}

Zur Bestimmung des Herzzeitvolumens und Erfassung der verschiedenen Flüssigkeitskompartimente erfolgte bei der vorliegenden Untersuchung eine simultane Applikation beider Indikatoren (vgl. Abschnitt 2.1). Zu jedem Meßpunkt wurden 22,5 $\mathrm{mg} I C G$ in $15 \mathrm{ml}$ Aqua ad injectionem gelöst, die Lösung im Eiswasserbad auf $0{ }^{\circ} \mathrm{C}$ abgekühlt und anschließend manuell als Bolus in das proximale Lumen des Pulmonaliskatheters injiziert (s. Abbildung 3-1). Es wurden immer Dreifachmessungen durchgeführt, wobei der Mittelwert von den drei aufeinanderfolgenden Messungen gebildet wurde.

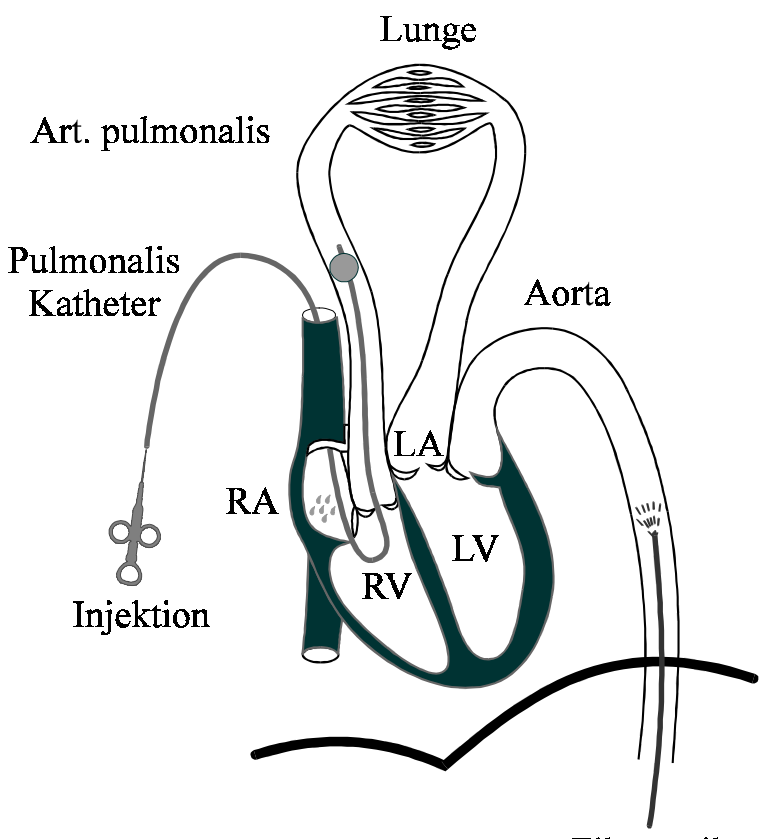

Fiberoptik-

Thermistor-Katheter

Abbildung 3-1. Zentralvenöse Bolusapplikation des Indikators in das proximale Lumen des Pulmonaliskatheters und Lage des aortalen Fiberoptik-Katheters

Die resultierenden Indikatordilutionskurven wurden mit einem handelsüblichen Hämoreflektometer $^{34}$, das eine simultane und verzögerungsfreie Erfassung der Thermo- und Farbstoffdilutionskurven von dem pulmonalen und aortalen Katheter ermöglichte, über vier

\footnotetext{
${ }^{34}$ Pulsion COLD Z-021 ${ }^{\circledR}$, Pulsion Medical Systems, München, BRD
} 
Minuten registriert. Die Temperaturänderungen wurden über die Thermistoren der plazierten Katheter, die Konzentrations-Zeitsignale des Farbstoffs über den Lichtleiter des aortalen Fiberoptik-Katheters, der mittels einer optoelektronischen Kopplung mit dem Reflektometer verbunden war, erfaßt.

Bei dem verwendeten Hämoreflektometer wird monochromatisches Licht der Wellenlänge $\lambda=805 \mathrm{~nm}$ (Absorptionsmaximum von $I C G$ ) und $\lambda=900 \mathrm{~nm}$ (Referenzwellenlänge) mit einer Frequenz von $300 \mathrm{~Hz}$ im Wechsel über einen Lichtleiter zur Katheterspitze eingekoppelt, wobei konvektive Einflüsse auf die Reflexionsintensitäten durch die gleichzeitige Messung an der Referenzwellenlänge eliminiert werden. Das Signal wird an die zirkulierenden Blutkörperchen reflektiert und anschließend über eine zweite optische Faser zu einer Photozelle transportiert, wo die Lichtintensität gemessen wird. Diese Signale werden zeitsynchronisiert, den beiden Wellenlängen zugeordnet und analog dividiert. Eine zur Indikatorkonzentration proportionale Analogspannung steht dann als Ausgangssignal zur Verfügung (s. Abbildung 3-2).

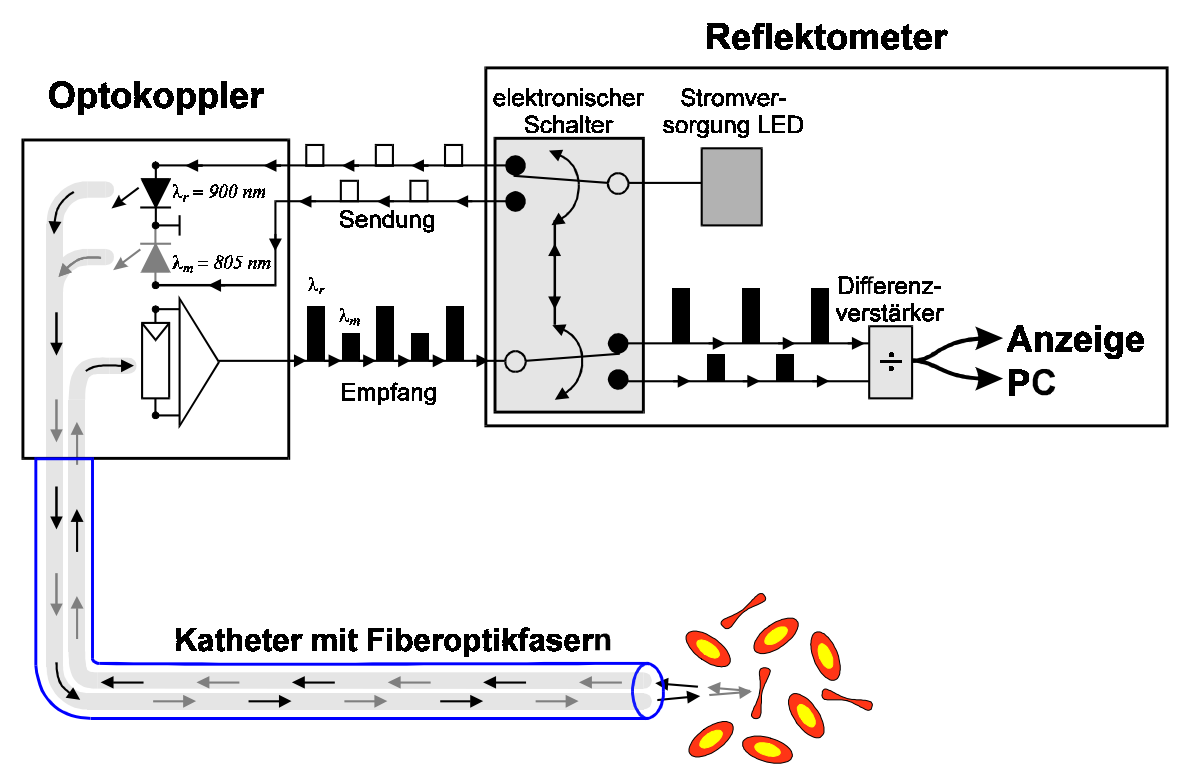

Abbildung 3-2. Aufbau des verwendeten Hämoreflektometers zur Erfassung der Farbstoffdilutionskurven (nach Wietasch 1995, S. 26)

Die analogen Ausgangssignale wurden über einen Analog-Digitalwandler digitalisiert und auf der Festplatte eines IBM-kompatiblen Personal Computers gespeichert. Die zur Aufzeichnung und zur Auswertung der Meßdaten benutzte Software wurde in der Programiersprache Borland Pascal $^{\circledR 35}$ geschrieben und zu einem maschinenlesbaren Code kompiliert. Die Auswertung der Daten erfolgte ebenfalls mit einem handelsüblichen Personal Computer.

\footnotetext{
${ }^{35}$ Borland Pascal $^{\circledR}$ version 7.0, Borland, München
} 


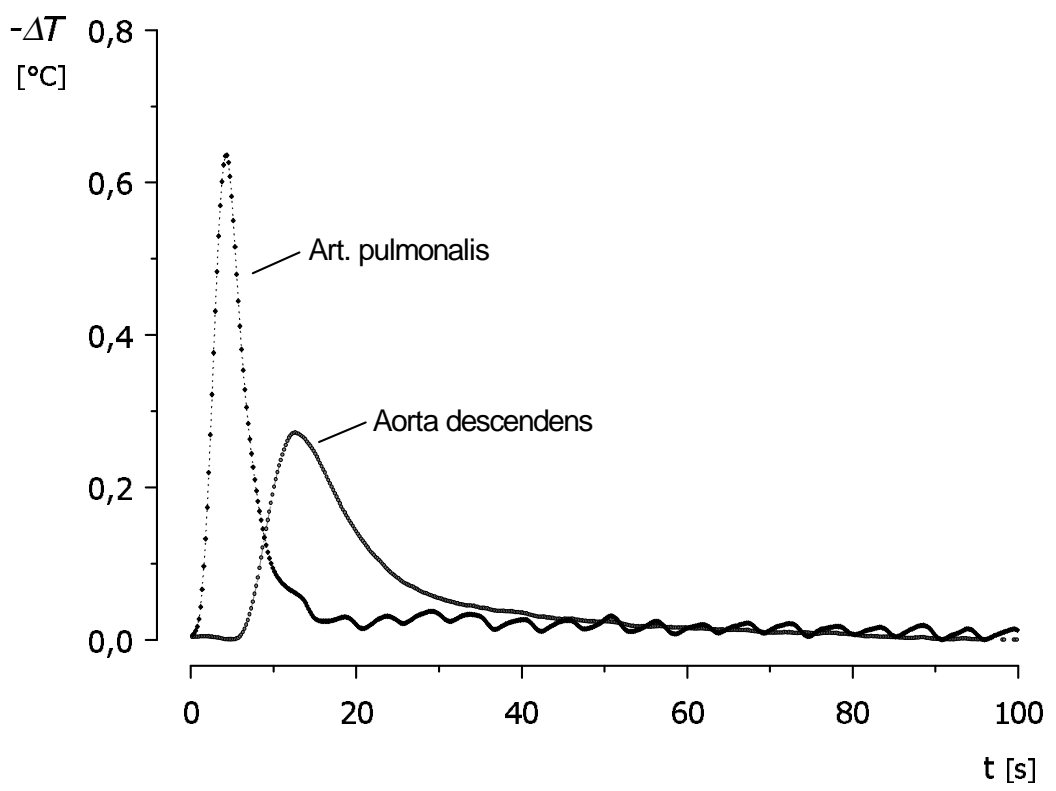

Abbildung 3-3. Pulmonal und aortal registrierte Thermodilutionskurven

$\mathrm{Zu}$ jeder Messung nach zentralvenöser Bolusapplikation des Indikators wurden je eine pulmonale und aortale Thermodilutionskurve und eine aortale Farbstoffverdünnungskurve simultan durch das Pulsion-COLD ${ }^{\circledR}$-Gerät registriert (s. Abbildung 3-3 und Abbildung 3-4). Die Auswertung der aufgezeichneten Kurven und die Berechnung der weiteren Parameter erfolgte nach den Prinzipien der Doppelindikatorverdünnungsmethode, die in dem Abschnitt Theoretische Grundlagen beschrieben wurde.

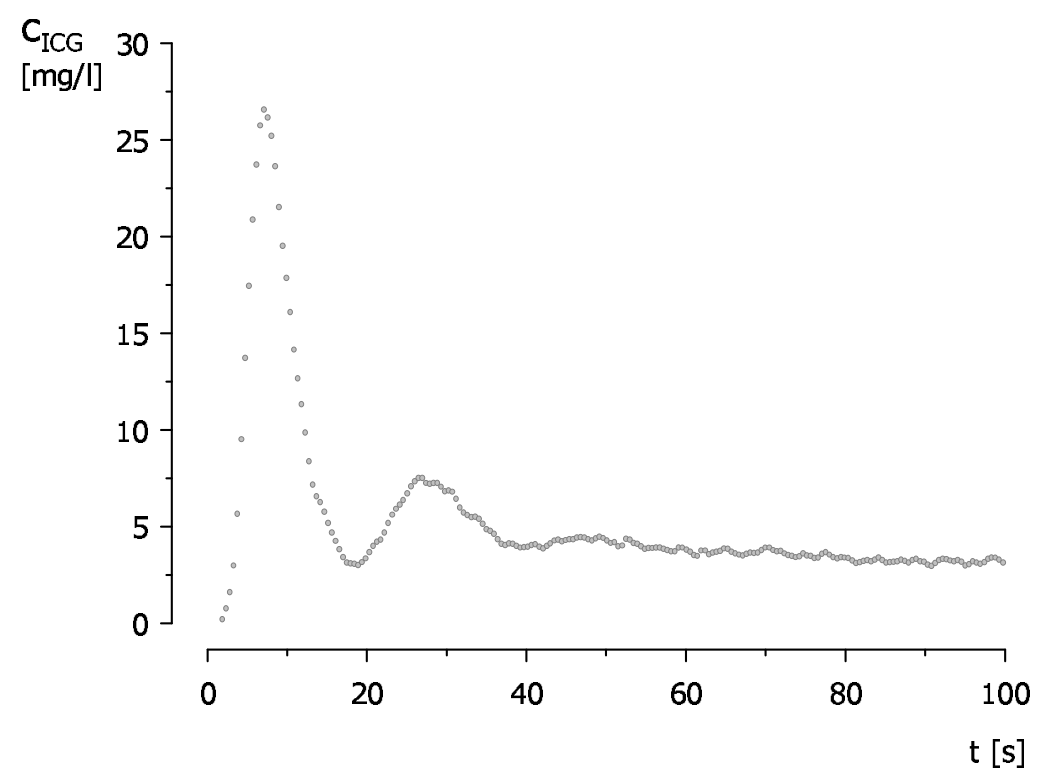

Abbildung 3-4. Aortal registrierte Farbstoffdilutionskurve 


\subsection{4 „In vitro“-Bestimmung des ,gesamten“ Blutvolumens}

Die Bestimmung des „gesamten“ Plasmavolumens erfolgte entsprechend der allgemein anerkannten konventionellen Methode (Schad et al. 1987; Haller et al. 1992; Grainger et al. 1983) aus der hepatischen Eliminationsrate für ICG. Eine Modifizierung des Verfahrens zur Ermittlung der Kalibrationskurve hatte eine noch empfindlichere spektrophotometrische Erfassung der Farbstoffkonzentration besonders bei niedrigen Indikatormengen zur Folge.

$\mathrm{Zu}$ den Meßzeitpunkten $n . N ., 1 h$, $6 h$ und $20 h$ erfolgte, nach Entnahme einer gemischtvenösen Blutprobe (in 8,5 ml Na-EDTA-Monovette) zur Hämatokritbestimmung und Gewinnung von Leerwert- und Eichplasma, die zentralvenöse Bolusapplikation von 22,5 mg ICG (vgl. Abschnitt 3.4.3). Als Zeitpunkt 0 der Messung wurde der Beginn der Injektion definiert. Nach 5, 6, 7, 8, 9 , 12, 15, 18, 21, 24, 27 und 30 Minuten wurden gemischtvenöse Blutproben in $\mathrm{Na}^{+}$-EDTAMonovetten abgenommen. Als Vorlauf wurden immer $3 \mathrm{ml}$ Blut über $4 \mathrm{sec}$ in mit Heparin benetzen Spritzen entnommen und später wieder reinjiziert. Um Kontaminationen mit Injektatresten auszuschließen, erfolgten die Blutentnahmen nicht aus dem Katheter, über den der Indikator appliziert wurde. Die Blutproben wurden zur Gewinnung von Plasma unmittelbar nach der Entnahme bei $4000 \mathrm{U} \cdot \mathrm{min}^{-1}$ über zehn Minuten zentrifugiert. Es wurde versucht, den Zeitraum zwischen ICG-Injektion und Belegung des Eichplasmas möglichst gering zu halten. ICG ist zwar im Plasma, jedoch nicht in wäßriger Lösung stabil (Björnsson et al. 1983).

Um die Präzision der Methode zu verbessern, wurde eine Spektralanalyse (Hoeft et al. 1994) anstatt einer Mono- oder Zweiwellenlängeanalyse (Schad et al. 1987) bevorzugt. Die Extinktion der Plasmaproben wurde über das gesamte Spektrum $(600-900 \mathrm{~nm})$ hinaus mit Hilfe eines Spektralphotometers $^{36}$ gegen bidestilliertes Wasser gemessen (s. Abbildung 3-5), wobei als Probengefäße Halbschichtmikroküvetten ${ }^{37}$ verwendet wurden. Die registrierten Spektren wurden auf der Festplatte eines MS-DOS ${ }^{\circledR}$-kompatiblen Rechners für eine spätere Bearbeitung gespeichert.

$\mathrm{Zu}$ jedem Meßpunkt und für jede einzelne Plasmavolumenberechnung wurde eine eigene Eichung erstellt, denn jede Indikatordosis wurde aus einer anderen Stammlösung hergestellt. Die Eichung erfolgte durch Zugabe von $20 \mu 1$ einer zuvor angesetzten Verdünnungsreihe (der zur Injektion verwendeten ICG-Lösung) zu je $980 \mu$ l Leerwertplasma mittels einer kalibrierten $10 \mu 1$-Pipette ${ }^{4}$, entsprechend Konzentrationen von 0,375, 0,75, 1,5 und $3 \mathrm{mg} \cdot \mathrm{l}^{-1}$. Zu den 4 erfaßten Eichwerten (entsprechen 8 Spektren beim Doppelbestimmungsverfahren) wurde der Leerwert als fünfter Wert mitberücksichtigt. Alle registrierten Spektren wurden ebenfalls auf der Festplatte des Personalcomputers gespeichert.

\footnotetext{
${ }^{36}$ Milton-Roy (MR-3000), USA

${ }^{37}$ Sarstedt Aktiengesellschaft \& Co, Nümbrecht, BRD
} 


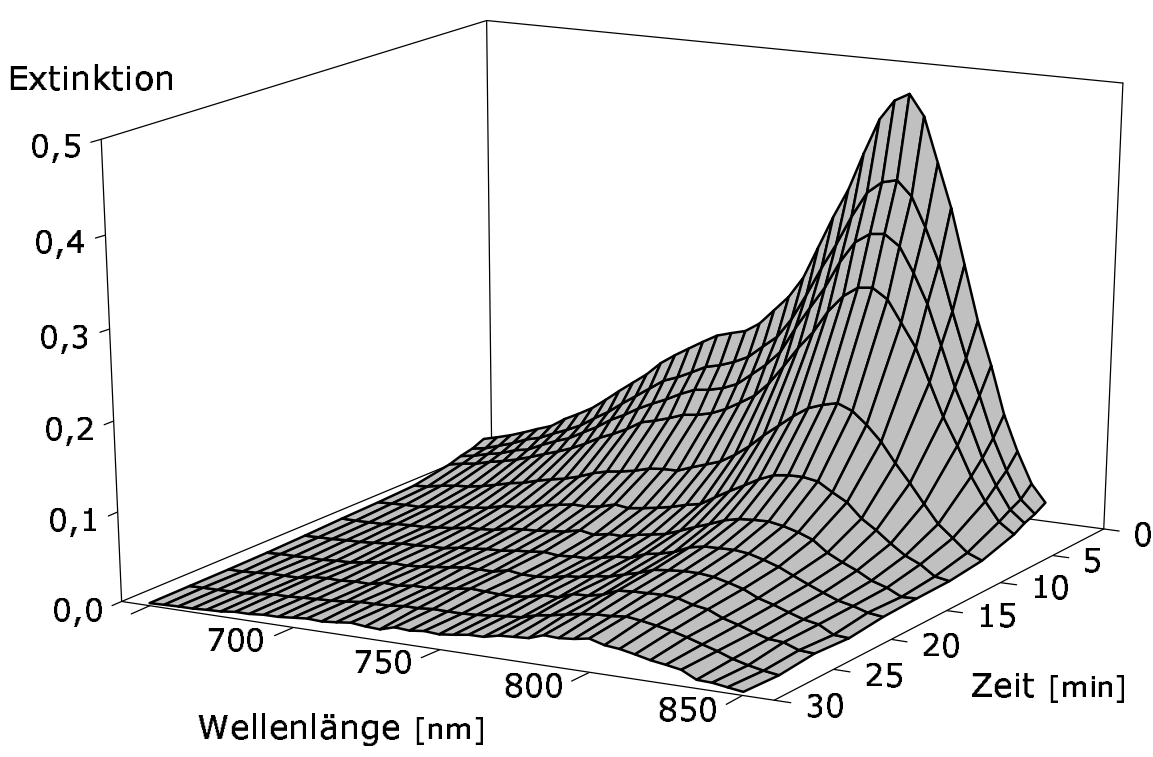

Abbildung 3-5. Die Extinktionen der abgenommenen Plasmaproben im Spektrumbereich zwischen 600 und $900 \mathrm{~nm}$ gegen bidestilliertes Wasser

Ziel der vorliegenden Untersuchungen war ein optimiertes Verfahren zu entwickeln, das auf die Prinzipien der im Kapitel 5.4.1 beschriebenen Methoden von M.H. Nielsen und N.C. Nielsen (Nielsen und Nielsen 1962; Nielsen 1963a; Nielsen 1963b) und Schad (1987) basiert, damit die Konzentration des ICGs auch bei sehr niedrigen Bereichen präzis bestimmt werden kann. Bei der Auswertung der Absorptionspektren wurde die Plasmaeigenextinktion berücksichtigt. Jedes aufgenommene Spektrum wurde gegenüber dem Leerwertspektrum an den Fußpunkten der Kurve bei 600 und $900 \mathrm{~nm}$, wo $I C G$ keine meßbare Absorption von Licht zeigt, korrigiert. Bei diesen Bereichen des Absorptionsspektrums zeigen darüber hinaus weder das für die Farbe verantwortliche Pigment der Galle noch Hämoglobin eine meßbare Lichtabsorption, und die Eigenabsorption vom Humanplasma ist ziemlich niedrig. Um Änderungen der Plasmaeigenextinktion und interindividuelle Unterschiede der Plasmaeigenschaften zu eliminieren, wurde die Leerwertkorrektur für jedes Experiment eigens erstellt.

\subsubsection{Berechnung der Indikatorkonzentration der Plasmaproben}

Um die Plasmakonzentration des Farbstoffes bei den abgenommenen Plasmaproben zu bestimmen wurde ein Kalibrationsspektrum erstellt. Für jeden Meßzeitpunkt und von allen Eichproben wurde für jede Wellenlänge von 605 bis $870 \mathrm{~nm}$ alle $0,94 \mathrm{~nm}$ eine lineare Regression auf der Basis der kleinsten Abstandsquadrate (Deuflhard und Apostolescu 1980) zwischen den Extinktionen $\left(E_{\lambda}\right)$ der Eichproben und ihren angegebenen ICG-Konzentrationen durchgeführt. Das Ergebnis der Regressionsanalyse ergab ein Spektrum von 282 Absorptionskoeffizienten für den Bereich zwischen 605 und $870 \mathrm{~nm}$ (Abbildung 3-6), wobei jeder Extinktionskoeffizient $\left(\varepsilon_{\lambda}\right)$ der Steigung der Regressionsgerade an der bestimmten Wellenlänge $(\lambda)$ entspricht. 


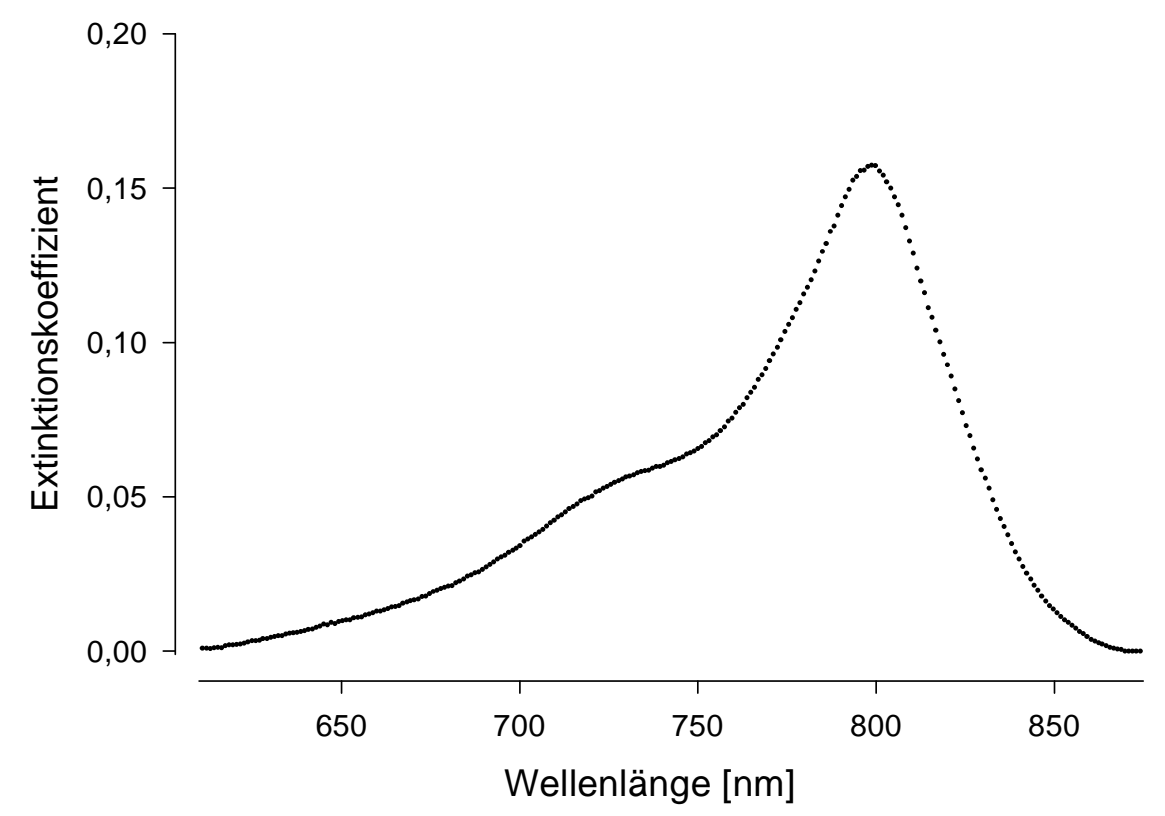

Abbildung 3-6. Beispiel eines Extinktionskoeffizientenspektrums, das als Kalibrationsspektrum zur Berechnung der ICG-Konzentration der Blutproben zu jedem Meßzeitpunkt diente.

Die Berechnung der ICG-Plasmakonzentrationen erfolgte dann mittels eines multiplen Linearregressionverfahrens, wobei die Extinktionsspektren der $I C G$-haltigen Plasmaproben an diesem Extinktionskoeffizientenspektrum angepaßt wurden. Die Plasmaindikatorkonzentration entsprach hierbei der Steigung der Regressionsgerade durch die gegen den Koeffizienten jeder Wellenlänge aufgetragenen Extinktionen des Blutprobenspektrums, zufolge des Bouguer-Lambert-Beer'schen Gesetzes:

\section{Gleichung 3-23}

$$
\begin{aligned}
& E_{\lambda}=\varepsilon_{\lambda} \cdot c \cdot d \\
& E_{\lambda} \quad=\text { die Extinktion an der Wellenlänge } \lambda \\
& \varepsilon_{\lambda} \quad=\text { der Extinktionskoeffizient der Wellenlänge } \lambda \\
& c \quad=\text { die gesuchte Konzentration } \\
& d \quad=\text { die Schichtdicke der Küvette (normiert) }
\end{aligned}
$$

Durch Verwertung des gesamten Spektrums wird das Signal-Rausch-Verhältnis, das die Streuung der einzelnen Korrelationswerten um die Regressionsgerade darstellt, im Vergleich zu der Monooder Zweiwellenlängenmethode minimiert. Dadurch wird eine verbesserte Kompensation für die Hintergrundabsorption der Plasmaproben erreicht. Nach Anderson (1989) lag die untere Indikatornachweisgrenze dieses Verfahrens bei $0,2 \mathrm{ml} \cdot 1^{-1}$ bei einem Variationskoeffizienten von $4 \%$ oder weniger für alle Konzentrationen.

\subsubsection{Berechnung des Verteilungvolumens nach dem Prinzip der Massenerhaltung}

Im Gegensatz zur Messung des „zirkulierenden“ Blutvolumens, die auf der Analyse des Verteilungsprozesses des Indikators im Transportmedium basiert (s. Abschnitt 2.6), wobei die erste Verteilungsphase nach der Farbstoffinjektion von Bedeutung ist, liegt bei der Bestimmung 
des „gesamten“ Blutvolumens das Prinzip der Massenerhaltung zugrunde. Das $V_{\text {d-ges }}$ berechnet sich demzufolge aus der im Organismus befindlichen applizierten Menge $m_{0}$ des Indikators und seiner Plasmakonzentration $c_{0}$ (Gleichung 2-2, S. 5).

Bei den meisten Indikatoren läuft nach der Applikation zunächst eine Verteilungsphase durch, bevor eine ausreichende Durchmischung in ihrem Verteilungsvolumen erreicht wird. So ist bis zum Zeitpunkt dieser ausreichenden Durchmischung bereits ein Teil des Farbstoffes eliminiert worden. Dementsprechend kann diese „,virtuelle“ $c_{0}$-Konzentration nicht direkt bestimmt werden, sondern muß aus der Eliminationskurve rückgerechnet werden. Basierend auf dem im Abschnitt 3.4.4 beschriebenen Verfahren wurde deshalb für jeden Meßzeitpunkt der KonzentrationsZeitverlauf bis 30 Minuten nach Applikation des Indikators ermittelt. Unter Zugrundelegen eines zweikompartimentellen pharmakokinetischen Modells und angesichts der genügend langen Meßdauer und der ausreichenden Meßgenauigkeit des Verfahrens wurde an die ermittelten Konzentrationswerten von ICG iterativ durch ein nichtlineares Verfahren auf der Basis der kleinsten Abstandsquadrate (Deuflhard und Apostolescu 1980) eine biexponentielle Modellfunktion der folgenden Form angepaßt (vgl. Abbildung 3-7):

\section{Gleichung 3-24}

$$
\begin{aligned}
& c(t)=a \cdot e^{-k_{1} \cdot t}+\beta \cdot e^{-k_{2} \cdot t} \\
& c(t)=\text { Die Konzentration des Farbstoffes für den Zeitpunkt } t \\
& \alpha, \beta=\text { Gewichtungsfaktoren für die Eliminationen des schnellen und des langsamen } \\
& \text { Kompartimentes } \\
& k_{1}, k_{2}=\text { Eliminations-Zeitkonstanten des schnellen und des langsamen Kompartimentes }
\end{aligned}
$$

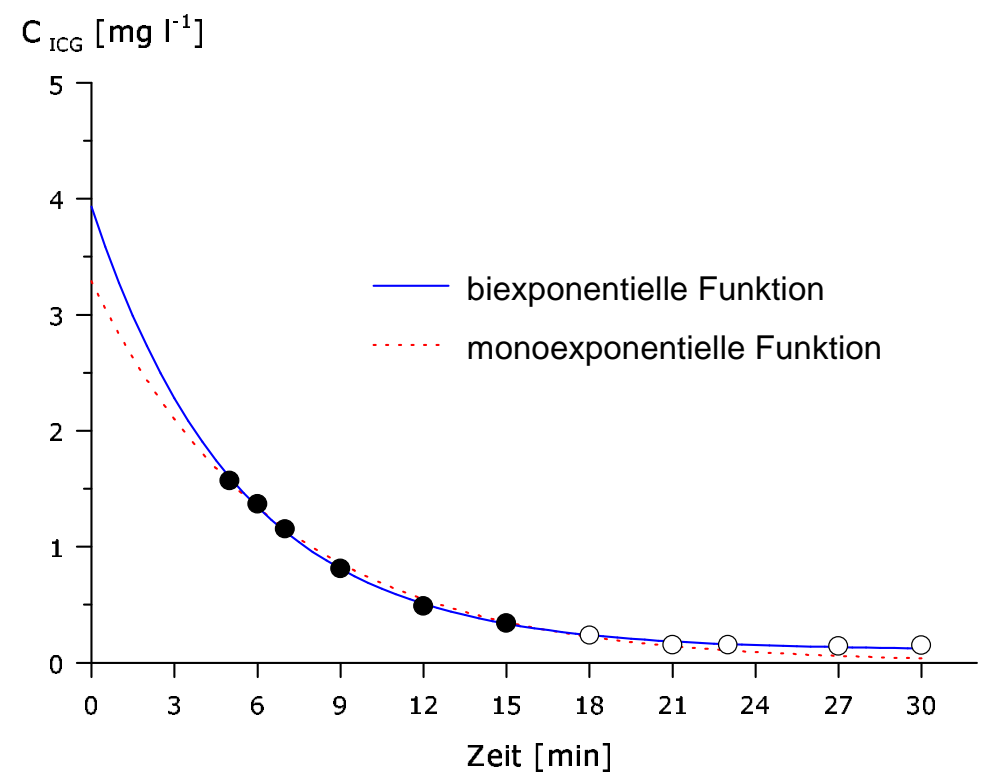

Abbildung 3-7. Konzentrations-Zeitverlauf von ICG; Darstellung der angepaßten Modellfunktionen auf der Basis von ein- und zweikompartimentellem Eliminationsmodell 
Die auf dieser Weise entstandene Eliminationskurve wurde aber nicht auf den Punkt der Bolusinjektion zurückextrapoliert, sondern auf den Zeitpunkt der ersten Leberpassage, da erst zu diesem Zeitpunkt die Elimination des Indikators beginnt (Schorn 1995; Haller et al. 1992; Haneda und Horiuchi 1986). Nach Schorn (1995) wurde dieser Zeitpunkt der ersten Leberpassage, der nahezu dem Erscheinungszeitpunkt des Farbstoffes in der peripheren Zirkulation entspricht (Haller et al. 1992), als $t_{e}=t_{0}+\frac{m t t_{f-z i r k}}{2}$ definiert. Demzufolge kann für $t=t_{e}$ die Gleichung 3-24 folgenderweise umformuliert werden:

\section{Gleichung 3-25}

$$
c_{e}=c\left(t_{e}\right)=\alpha \cdot e^{-k_{1} \cdot \frac{m t t_{f-i i r k}}{2}}+\beta \cdot e^{-k_{2} \cdot \frac{m t_{f-z i r k}}{2}}
$$

Das „korrigierte“ Plasmavolumen wird also berechnet, wenn wir in der Gleichung 2-2 die Konzentration $c_{\mathrm{e}}$ anstatt der $c_{0}$ einsetzen. Aus dieser Beziehung und unter Berücksichtigung der Unterscheidung von „Ganzkörperhämatokrit“ und dem „Hämatokrit in großen Gefäßen“ (Swan und Nelson 1971) wurde der Hämatokrit für ,trapped plasma“ korrigiert (Reeve und Veall 1949) und das gesamte Blutvolumen nach der folgenden Formel berechnet:

\section{Gleichung 3-26}

$$
V_{d-\text { ges }}=\frac{V_{d-\text { plasma }}}{0,87 \cdot\left(1-\frac{H k t}{100}\right)}
$$

\subsection{Statistische Datenanalyse ${ }^{38}$}

Als deskriptive Verfahren wurden in der vorliegenden Studie zur Beschreibung der Ergebnisse die Mittelwerte $(\bar{x})$ mit ihren empirischen Standardabweichungen $(s d)$ angegeben. Bei der grafischen Darstellung wurde zugunsten einer besseren Übersichtlichkeit als Streuungsmaß der Standardfehler des Mittelwertes (sem) benutzt.

Die statistische Auswertung erfolgte mit einem üblichen Statistikprogramm ${ }^{39}$, wobei ein signifikanter Unterschied bei einer Irrtumswahrscheinlichkeit von weniger als $5 \%(p<0,05)$ angenommen wurde.

Zur Überprüfung von statistisch nachweisbaren Unterschieden zwischen den untersuchten Patientengruppen hinsichtlich ihrer biometrischen Daten, kam der Kruskal-Wallis- bzw. der $\chi^{2}$-Test zur Anwendung (Tabelle 4-1).

Die statistische Prüfung der Mittelwerte der übrigen Parameter auf Verschiedenheit erfolgte mit Hilfe einer Varianzanalyse (ANOVA) für wiederholte Messungen. Bei statistisch signifikanten

\footnotetext{
${ }^{38}$ Die Beratung zur statistischen Ausarbeitung der Ergebnisse erfolgte durch das Institut für Medizinische Statistik der Georg-August-Universität Göttingen (Prof. Dr. E. Brunner).

${ }^{39}$ Statistica ${ }^{\circledR}$ for Windows, Release version 4.0, ( 1993 by Statsoft Inc., Tulsa/OK, USA
} 
Unterschieden wurde anschließend ein post hoc-Vergleich innerhalb und zwischen den Patientengruppen durchgeführt, unter Zugrundelegen einer zweiseitigen Irrtumswahrscheinlichkeit von $5 \%$.

Der Zusammenhang zwischen zwei Meßgrößen wurde zunächst mittels einer linearen Regressionsanalyse zur Bestimmung der Parameter der Regressionsgerade und des Korrelationskoeffizienten ( $r$ ) überprüft. Zur statistischen Bewertung der Ergebnisse im Sinne eines Methodenvergleichs wurde jedoch zusätzlich die deskriptive Darstellung nach Altman und Bland (1983) angewendet. Bei der graphischen Darstellung erfolgt die Auftragung des Mittelwertes der beiden Methoden (Abszisse) gegenüber der Differenz zwischen den beiden Methoden (Ordinate). Die mittlere Abweichung zwischen den untersuchten Methoden stellt ein Maß für den systematischen Fehler, die zweifache Standardabweichung der Methodendifferenz ein Maß für die Präzision dar. 


\section{Ergebnisse}

Nach Zustimmung der hiesigen Ethikkomission und schriftlichem Einverständnis der Patienten wurden die vorliegenden Untersuchungen an insgesamt 20 erwachsenen Patienten durchgeführt, die sich einer elektiven Myokardrevaskularisation unterziehen mußten. Bei den 20 Patienten wurden hämodynamische Daten, Ein- und Ausfuhrbilanzen sowie Indikatordilutionskinetiken zur quantitativen Erfassung verschiedener Flußraten und Flüssigkeitskompartimente erhoben. Die Messungen wurden unmittelbar nach Narkoseeinleitung (n.N.) sowie eine Stunde (lh), sechs Stunden (6h) und zwanzig Stunden (20h) nach Operationsende $(O p E)$ durchgeführt. Nach Einleitung der Narkose erfolgte unter doppel-blind kontrollierten Bedingungen die Applikation der Testsubstanz.

\subsection{Biometrische Daten der Patienten}

Die Gruppe der Patienten, bei denen nach Einleitung der Narkose Dexamethason appliziert wurde, unterschied sich nicht wesentlich von der Kontrollgruppe bezüglich Alter, Ischämie- und Perfusionszeit während der extrakorporalen Zirkulation sowie Blutverlust und Autotranfusionsmenge (s. Tabelle 4-1). Dennoch war der Anteil der männlichen Patienten in der DexamethasonGruppe höher, so daß signifikante Unterschiede hinsichtlich der Körpergröße, des Körpergewichts und der Körperoberfläche auftraten. Um die ermittelten Daten zu standardisieren und vergleichbare Werte mit anderen wissenschaftlichen Arbeiten zu erhalten, wurden alle Angaben zu Flüssigkeitsräumen, Flußarten und Bilanzen auf das Körpergewicht oder die Körperoberfläche bezogen.

\begin{tabular}{lccc}
\hline & PLC & & DXM \\
\hline Größe [cm] & $165 \pm 6$ & $* *$ & $173 \pm 6$ \\
Gewicht [kg] & $70,6 \pm 10$ & $*$ & $85,5 \pm 11$ \\
Körperoberfläche [m²] & $1,78 \pm 0,15$ & $* *$ & $1,99 \pm 0,13$ \\
Alter [Jahre] & $66,8 \pm 3,7$ & & $62,5 \pm 9,5$ \\
Geschlecht (m/w) & $6 / 4$ & & $9 / 1$ \\
Perfusionszeit [min] & $101 \pm 34$ & & $103 \pm 39$ \\
Ischämiezeit [min] & $65 \pm 25$ & & $67 \pm 28$ \\
Cellsaver [ml] & $871 \pm 447$ & & $738 \pm 314$ \\
Blutverlust [ml] & $1369 \pm 357$ & $1599 \pm 713$ \\
\hline
\end{tabular}

Tabelle 4-1. Biometrische Daten der Patienten ( $\bar{x} \pm$ sd)

PLC $=$ Placebo-Gruppe $(\mathrm{n}=10) ;$ DXM $=$ Dexamethason-Gruppe $(\mathrm{n}=10)$

$*(* *): \mathrm{p}<0,05(0,01):$ Kruskal-Wallis- bzw. $\chi^{2}$-test

\subsection{Laborchemische Parameter}

In der Tabelle 4-2 sind die perioperativen Verläufe der wichtigsten laborchemischen Parameter dargestellt. Die Ausgangswerte der Parameter waren in den zwei Untersuchungsgruppen vergleichbar. Jedoch unterschieden sich präoperativ die Werte für das Gesamtprotein $(T P)$ in den 
Gruppen signifikant ohne eine faßbare Abweichung bezüglich der präoperativen Vorbereitung der Patienten.

\begin{tabular}{|c|c|c|c|c|c|}
\hline & & n.N. & $1 \mathrm{~h}$ & $6 \mathrm{~h}$ & $20 \mathrm{~h}$ \\
\hline $\begin{array}{c}\mathbf{H b} \\
{\left[\mathrm{g} \cdot \mathrm{dl}^{-1}\right]}\end{array}$ & $\begin{array}{l}\text { PLC } \\
\text { DXM }\end{array}$ & $\begin{array}{l}10,4 \pm 0,9 \\
11,0 \pm 1,8\end{array}$ & $\begin{array}{r}10,7 \pm 1,8 \\
9,8 \pm 0,9\end{array}$ & $\begin{array}{r}9,2 \pm 1,9 \\
10,0 \pm 0,7\end{array}$ & $\begin{array}{l}10,7 \pm 1,7 \\
10,2 \pm 1,3\end{array}$ \\
\hline $\begin{array}{l}\text { Hkt } \\
{[\%]}\end{array}$ & $\begin{array}{l}\text { PLC } \\
\text { DXM }\end{array}$ & $\begin{array}{l}31,9 \pm 2,7 \\
34,5 \pm 5,4\end{array}$ & $\begin{array}{l}30,9 \pm 5,9 \\
29,3 \pm 2,5 *\end{array}$ & $\begin{array}{l}29,7 \pm 5,2 \\
30,5 \pm 2,5\end{array}$ & $\begin{array}{l}32,6 \pm 4,2 \\
30,9 \pm 4,1\end{array}$ \\
\hline $\begin{array}{c}\text { WBC } \\
{\left[\times\left. 10^{9} \cdot\right|^{-1}\right]}\end{array}$ & $\begin{array}{l}\text { PLC } \\
\text { DXM }\end{array}$ & $\begin{array}{l}6,1 \pm 2,1 \\
5,9 \pm 1,0\end{array}$ & $\begin{array}{l}11,9 \pm 4,1 * * \\
12,1 \pm 3,9 * *\end{array}$ & $\begin{array}{l}11,9 \pm 3,1 * * \\
11,5 \pm 2,9 * *\end{array}$ & $\begin{array}{l}12,2 \pm 2,1 * * \\
13,4 \pm 4,3^{* *}\end{array}$ \\
\hline $\begin{array}{c}\text { PLT } \\
{\left[\times\left. 10^{9} \cdot\right|^{-1}\right]}\end{array}$ & $\begin{array}{l}\text { PLC } \\
\text { DXM }\end{array}$ & $\begin{array}{l}179,6 \pm 36,9 \\
192,2 \pm 39,2\end{array}$ & $\begin{array}{l}123,9 \pm 30,9 * * \\
109,2 \pm 28,2 * *\end{array}$ & $\begin{array}{l}136,6 \pm 42,4 * \\
124,2 \pm 54,3^{*} *\end{array}$ & $\begin{array}{l}129,8 \pm 34,3^{*} \\
116,3 \pm 19,5^{* *}\end{array}$ \\
\hline $\begin{array}{c}\text { Glc } \\
{\left[\mathrm{mg} \cdot \mathrm{dl}^{-1}\right]}\end{array}$ & $\begin{array}{l}\text { PLC } \\
\text { DXM }\end{array}$ & $\begin{array}{r}108,6 \pm 14,9 \\
99,5 \pm 19,9\end{array}$ & $\begin{array}{l}156,4 \pm 41,8^{*} \\
175,1 \pm 35,1^{*}\end{array}$ & $\begin{array}{l}199,2 \pm 37,2^{* *} \\
205,8 \pm 46,0^{* *}\end{array}$ & $\begin{array}{l}185,6 \pm 69,7^{* *} \\
185,6 \pm 46,7^{* *}\end{array}$ \\
\hline $\begin{array}{c}\text { TP } \\
{\left[\left.\mathrm{g} \cdot \mathrm{d}\right|^{-1}\right]}\end{array}$ & $\begin{array}{l}\text { PLC } \\
\text { DXM }\end{array}$ & $\begin{array}{c}\# \\
4,91 \pm 0,48 \\
5,78 \pm 0,66\end{array}$ & $\begin{array}{l}3,81 \pm 0,24 * * \\
4,11 \pm 0,54 * *\end{array}$ & $\begin{array}{c}\# \# \\
3,76 \pm 0,36 * * \\
4,27 \pm 0,44^{* *}\end{array}$ & $\begin{array}{l}4,41 \pm 0,50 * * \\
4,61 \pm 0,54 * *\end{array}$ \\
\hline
\end{tabular}

Tabelle 4-2. Laborchemische Parameter ( $\bar{x} \pm \mathrm{sd})$

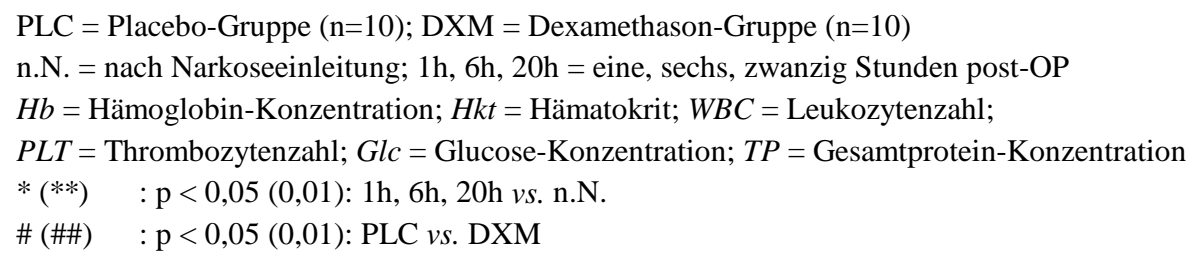

Der Hämoglobingehalt $(H b)$ und der Hämatokrit $(H k t)$ zeigten während des gesamten postoperativen Verlaufs keine wesentliche Änderung im Vergleich zu den Ausgangsmessungen nach Einleitung der Narkose. Die Leukozytenzahl $(W B C)$ und die Glucose-Konzentration des Plasmas $(G l c)$ wiesen postoperativ von der ersten bis zur zwanzigsten Stunde einen signifikanten Anstieg auf, ohne statistisch relevanten Unterschiede zwischen den beiden Patientengruppen. Demgegenüber fand sich postoperativ ein signifikanter Abfall der Thrombozytenzahl, etwas ausgeprägter in der Dexamethason-Gruppe. Der Gesamtproteingehalt (TP) fiel postoperativ in den beiden Gruppen ebenfalls ab. Sechs Stunden (6h) nach Ende der Operation war ein statistisch signifikanter Unterschied zwischen Placebo- und Dexamethason-Gruppe nachweisbar, allerdings bei präoperativ ungleichen Ausgangswerten.

\subsection{Hämodynamische Kreislaufparameter}

In Tabelle 4-3 sind die für die beiden Gruppen zu den verschiedenen Meßzeitpunkten ermittelten hämodynamischen Kreislaufparameter aufgeführt. Die Ausgangswerte waren in den Untersuchungsgruppen vergleichbar. 
In der postoperativen Phase fand sich in beiden Gruppen ein Anstieg der Herzfrequenz $(H f)$ und des Herzzeitvolumensindex $(C I)$ im Vergleich zu den präoperativ ermittelten Werten. Der Anstieg des Herzzeitvolumensindex war bei der Placebo-Gruppe in der frühen postoperativen Phase deutlicher, erreichte nach 20 Stunden jedoch wieder ein Niveau unterhalb dem der Dexamethason-Patienten. Der zentraler Venendruck $(Z V D)$ zeigte zu den Meßzeitpunkten und zwischen den Gruppen keine statistisch relevante Änderung, allerdings lagen die Werte ein Tag nach der Operation unter den Ausgangsniveau.

Ein signifikanter Unterschied zwischen den Untersuchungsgruppen fand sich bei den systolischen $(A P s)$ und den diastolischen $(A P d)$ arteriellen Blutdruckwerten eine Stunde $(1 h)$ nach der Operation. Diese stiegen bei der Placebo-Gruppe in der ersten postoperativen Stunde genauso wie der arterieller Mitteldruck $(M A P)$ signifikant an und erreichten nach 20 Stunden wieder niedrigere Werte.

\begin{tabular}{|c|c|c|c|c|c|}
\hline & & n.N. & $1 \mathrm{~h}$ & $6 \mathrm{~h}$ & $20 \mathrm{~h}$ \\
\hline \multirow{2}{*}{$\underset{\left[\mathrm{min}^{-1}\right]}{\mathbf{H f}}$} & PLC & $62,6 \pm 16,1$ & $91,4 \pm 7,8 * *$ & $92,2 \pm 16,7 * *$ & $87,7 \pm 20,6 * *$ \\
\hline & DXM & $61,3 \pm 11,4$ & $87,4 \pm 14,8^{* *}$ & $93,6 \pm 13,4 * *$ & $91,7 \pm 10,8 * *$ \\
\hline \multirow{3}{*}{$\begin{array}{c}\text { APs } \\
{[\mathrm{mmHg}]}\end{array}$} & & & \# & & \\
\hline & PLC & $105,9 \pm 18,8$ & $123,1 \pm 10,0 * *$ & $103,1 \pm 8,4$ & $116,2 \pm 19,6$ \\
\hline & DXM & $109,6 \pm 18,8$ & $111,6 \pm 17,0$ & $110,2 \pm 18,0$ & $115,6 \pm 17,7$ \\
\hline \multirow{3}{*}{$\begin{array}{c}\text { APd } \\
{[\mathrm{mmHg}]}\end{array}$} & & & \# & & \\
\hline & PLC & $52,5 \pm 7,7$ & $64,3 \pm 5,2 * *$ & $54,8 \pm 3,3$ & $57,5 \pm 10,3$ \\
\hline & DXM & $59,5 \pm 5,5$ & $58,7 \pm 8,1$ & $61,3 \pm 11,9$ & $58,6 \pm 8,5$ \\
\hline \multirow{2}{*}{$\begin{array}{c}\text { MAP } \\
{[\mathrm{mmHg}]}\end{array}$} & PLC & $70,4 \pm 11,7$ & $83,8 \pm 8,0 * *$ & $70,5 \pm 3,9$ & $76,3 \pm 12,2$ \\
\hline & DXM & $75,9 \pm 8,9$ & $78,1 \pm 14,7$ & $77,1 \pm 13,1$ & $77,0 \pm 10,6$ \\
\hline \multirow{3}{*}{$\begin{array}{l}\text { PAPs } \\
{[\mathrm{mmHg}]}\end{array}$} & & & \#\# & & \\
\hline & PLC & $25,1 \pm 6,5$ & $32,2 \pm 3,6 * *$ & $31,6 \pm 8,1 * *$ & $29,6 \pm 8,6$ \\
\hline & DXM & $22,6 \pm 6,1$ & $25,9 \pm 4,9$ & $27,5 \pm 5,5$ & $26,9 \pm 7,4$ \\
\hline \multirow{3}{*}{$\begin{array}{l}\text { PAPd } \\
{[\mathrm{mmHg}]}\end{array}$} & & & \#\# & \# & \# \\
\hline & PLC & $13,7 \pm 5,3$ & $18,2 \pm 3,3 * *$ & $17,4 \pm 2,9 *$ & $15,9 \pm 2,8$ \\
\hline & DXM & $11,6 \pm 5,4$ & $14,7 \pm 2,7$ & $14,3 \pm 2,7$ & $12,6 \pm 4,0$ \\
\hline \multirow{3}{*}{$\begin{array}{l}\text { MPAP } \\
{[\mathrm{mmHg}]}\end{array}$} & & & \#\# & \#\# & \\
\hline & PLC & $18,9 \pm 6,2$ & $23,9 \pm 2,2 * *$ & $23,2 \pm 3,9 * *$ & $21,4 \pm 3,7$ \\
\hline & DXM & $16,4 \pm 5,4$ & $19,1 \pm 3,0$ & $19,1 \pm 3,4$ & $18,2 \pm 4,4$ \\
\hline \multirow{2}{*}{$\begin{array}{c}\text { ZVD } \\
{[\mathrm{mmHg}]}\end{array}$} & PLC & $10,5 \pm 3,5$ & $10,9 \pm 2,1$ & $11,0 \pm 1,9$ & $9,1 \pm 2,8$ \\
\hline & DXM & $8,9 \pm 5,1$ & $9,7 \pm 3,9$ & $9,0 \pm 2,8$ & $6,9 \pm 3,4$ \\
\hline \multirow{3}{*}{$\begin{array}{l}\text { PCWP } \\
{[\mathrm{mmHg}]}\end{array}$} & & & & & \# \\
\hline & PLC & $12,2 \pm 5,0$ & $12,9 \pm 3,7$ & $13,1 \pm 2,5$ & $13,2 \pm 2,5$ \\
\hline & DXM & $11,9 \pm 5,5$ & $11,7 \pm 2,8$ & $11,9 \pm 2,4$ & $9,8 \pm 3,1$ \\
\hline \multirow{2}{*}{$\underset{\left[l \cdot \mathrm{min}^{-1} \cdot \mathrm{m}^{-2}\right]}{\mathrm{Cl}}$} & PLC & $2,3 \pm 0,6$ & $3,0 \pm 0,9 * *$ & $3,1 \pm 0,8 * *$ & $3,1 \pm 0,6 * *$ \\
\hline & DXM & $2,3 \pm 0,5$ & $2,7 \pm 0,7$ & $3,1 \pm 0,5 * *$ & $3,3 \pm 0,8^{* *}$ \\
\hline \multirow{2}{*}{$\underset{\left[\mathrm{SVI} \cdot \mathrm{m}^{-2}\right]}{\mathbf{S}}$} & PLC & $38,5 \pm 9,2$ & $33,1 \pm 8,7 *$ & $33,5 \pm 6,5 *$ & $35,5 \pm 4,3$ \\
\hline & DXM & $37,6 \pm 6,5$ & $31,3 \pm 7,0 * *$ & $33,7 \pm 5,1$ & $36,3 \pm 7,3$ \\
\hline
\end{tabular}




\begin{tabular}{|c|c|c|c|c|c|}
\hline & & & \#\# & \#\# & \\
\hline PVRI & PLC & $210,8 \pm 66,6$ & $311,6 \pm 176,3 * *$ & $257,9 \pm 86,6$ & $226,7 \pm 90,2$ \\
\hline $\begin{array}{l}\text { [dyn.sec. } \\
\mathrm{m}^{-2} \cdot \mathrm{cm}^{-5} \text { ] }\end{array}$ & DXM & $161,5 \pm 60,9$ & $205,8 \pm 56,9$ & $187,2 \pm 51,7$ & $193,9 \pm 42,8$ \\
\hline SVRI & PLC & $2137 \pm 533$ & $2102 \pm 722$ & $1636 \pm 438$ & $1804 \pm 557$ \\
\hline \multirow[t]{2}{*}{$\begin{array}{l}\text { [dyn.sec. } \\
\mathrm{m}^{-2} \cdot \mathrm{cm}^{-5} \text { ] }\end{array}$} & DXM & $2456 \pm 737$ & $2077 \pm 614$ & $1816 \pm 608^{* *}$ & $1777 \pm 670 * *$ \\
\hline & & & $\#$ & \#\# & \\
\hline $\mathrm{T}_{\mathrm{ao}}$ & PLC & $35,0 \pm 0,32$ & $36,1 \pm 0,72 * *$ & $37,4 \pm 0,52 * *$ & $36,9 \pm 0,41^{* *}$ \\
\hline$\left[{ }^{\circ} \mathrm{C}\right]$ & DXM & $35,4 \pm 0,69$ & $35,6 \pm 0,65$ & $36,8 \pm 0,66 * *$ & $36,9 \pm 0,39 * *$ \\
\hline
\end{tabular}

Tabelle 4-3. Hämodynamische Kreislaufparameter der Patienten ( $\bar{x} \pm \mathrm{sd})$

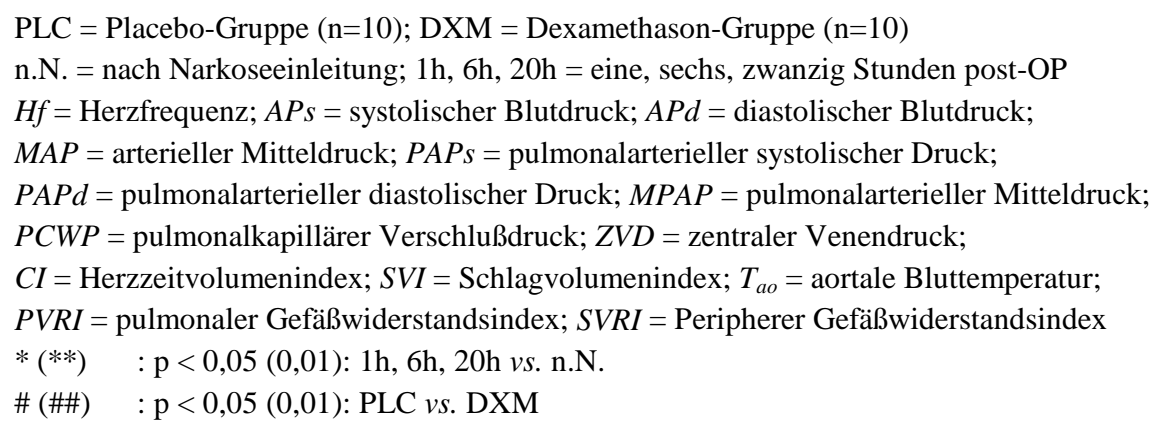

Patienten der Placebo-Gruppe wiesen $\mathrm{zu}$ allen Meßzeitpunkten im Mittel höhere pulmonalarterielle Drücke auf, obwohl unter Ausgangsbedingungen keine signifikanten Unterschiede bestanden. Zwischen den Zeitpunkten $1 h$ und $6 h$ nahmen der systolische (PAPS), der diastolische $(P A P d)$ und der mittlere $(M P A P)$ pulmonalarterielle Druck sowie der pulmonale Gefäßwiderstandsindex $(P V R I)$ bei den Patienten ohne präoperative Glucocortikoid-Medikation signifikant zu, um anschließend in der späten postoperativen Phase in einem geringeren Ausmaß wieder abzufallen. In dieser Phase zeigte die Dexamethason-Vorbehandlung einen signifikanten Einfluß auf den Verlauf der Drücke und der Gefäßwiderstände im kleinen Kreislauf. Bei Betrachtung des pulmonalkapillären Verschlußdruckes $(P C W P)$ konnte man in der PlaceboGruppe einen Anstieg bis zum ersten postoperativen Tag beobachten. Die Werte waren am Meßzeitpunkt $20 h$ noch deutlich über den Ausgangswerten und signifikant höher gegenüber den Werten der Dexamethason-Patienten.

Der periphere Gefäßwiderstandsindex (SVRI) fiel in beiden Gruppen im postoperativen Verlauf $\mathrm{ab}$ und zeigte eine maximale Senkung in der Placebo-Gruppe 6 Stunden und in der Dexamethason-Gruppe 20 Stunden nach der Operation. Der linksventrikuläre Schlagvolumenindex $(S V I)$ wies in den Gruppen ein nahezu ähnliches Verhalten mit deutlichem Abfall 1 bis 6 Stunden postoperativ auf.

Die aortal gemessene Bluttemperatur $\left(T_{a o}\right)$ stieg in beiden Gruppen postoperativ signifikant an. Maximalwerte erreichten die Placebo-Patienten zum Zeitpunkt $6 h$ und die DexamethasonPatienten zum Zeitpunkt 20h. Statistisch nachweisbare Gruppenunterschiede fanden sich eine und sechs Stunden nach der Operation. 


\subsection{Postoperativ applizierte Medikamente}

Die in der postoperativen Phase zwischen den einzelnen Meßpunkten verabreichten Medikamente sind in der Tabelle 4-4 dargestellt. Innerhalb der Untersuchungsgruppen unterlag der Medikamentenverbrauch großen individuellen Schwankungen. Jedoch erhielten Patienten, die mit Dexamethason vorbehandelt wurden, in der späten postoperativen Phase signifikant geringere Mengen an Adrenalin und Dopamin zur Unterstützung der myokardialen Inotropie. Der Diuretikaverbrauch lag im Mittel bei Dexamethason-Patienten etwas höher als bei der PlaceboGruppe, konnte dies allerdings aufgrund zu großer Streuungen innerhalb der Gruppen statistisch nicht gesichert werden. Die intra- und postoperative Applikation von Glyceroltrinitrat zur Senkung des Nachlasts oder zur Regulierung der venösen Kapazitätsgefäße und entsprechender Optimierung des myokardialen $\mathrm{O}_{2}$-Verbrauchs zeigte zwischen den Gruppen keine relevanten Unterschiede.

\begin{tabular}{|c|c|c|c|c|}
\hline & & n.N.-1h & n.N.-6h & n.N.-20h \\
\hline \multirow{3}{*}{$\begin{array}{c}\text { Adrenalin } \\
{[\mu g]}\end{array}$} & & & & \# \# \\
\hline & PLC & $275 \pm 304$ & $698 \pm 782$ & $1682 \pm 1991$ \\
\hline & DXM & $201 \pm 221$ & $470 \pm 393$ & $726 \pm 765$ \\
\hline \multirow{3}{*}{$\begin{array}{c}\text { Dopamin } \\
{[\mathrm{mg}]}\end{array}$} & & & & \#\# \\
\hline & PLC & $69 \pm 123$ & $321 \pm 517$ & $1199 \pm 1667$ \\
\hline & DXM & $21 \pm 66$ & $111 \pm 351$ & $345 \pm 1091$ \\
\hline \multirow{2}{*}{$\begin{array}{l}\text { NTG } \\
{[\mathrm{mg}]}\end{array}$} & PLC & $5,2 \pm 3,6$ & $14,7 \pm 2,5$ & $42,5 \pm 5,0$ \\
\hline & DXM & $5,8 \pm 4,3$ & $15,9 \pm 5,4$ & $40,7 \pm 8,3$ \\
\hline \multirow{2}{*}{$\begin{array}{l}\text { Furosemid } \\
{[\mathrm{mg}]}\end{array}$} & PLC & $10,5 \pm 18,0$ & $13,5 \pm 17,3$ & $30,5 \pm 39,5$ \\
\hline & DXM & $17,0 \pm 14,2$ & $18,0 \pm 15,5$ & $33,5 \pm 26,0$ \\
\hline
\end{tabular}

Tabelle 4-4. Kumulative Dosen applizierter Medikamente ( $\bar{x} \pm \mathrm{sd})$

PLC $=$ Placebo-Gruppe $(n=10) ;$ DXM = Dexamethason-Gruppe $(n=10)$

n.N. = nach Narkoseeinleitung; 1h, 6h, 20h = eine, sechs, zwanzig Stunden post-OP

$N T G=$ Glyceroltrinitrat

\#\# : p < 0,01: PLC vs. DXM 


\subsection{Flüssigkeitsverteilung im intrathorakalen Raum}

Für das „zentrale“ Blutvolumen und das „extravaskuläre Lungenwasser“ ergaben sich unter Ausgangsbedingungen keine signifikante Unterschiede (vgl. Abbildung 4-1 und Abbildung 4-2).

Im zeitlichen Verlauf zeigte das ,zentrale“ Blutvolumen $\left(V_{d \text {-zent }}\right)$ in der Dexamethason-Gruppe bereits eine Stunde nach Operationsende einen signifikanten Abfall (vgl. Abbildung 4-1). Zum Meßzeitpunkt $6 h$ lagen die Werte noch deutlich unter den Ausgangswerten, erreichten dann aber nach 20 Stunden mehr oder weniger den Ausgangsbereich. Eine Stunde nach der Operation wiesen die Placebo-Patienten einen weniger stark ausgeprägten Abfall des $V_{\text {d-zent }}$ auf. Im weiteren postoperativen Verlauf stieg das ,zentrale“ Blutvolumen erneut an und zum Zeitpunkt 20h lagen die Werte etwas über dem Ausgangsniveau.
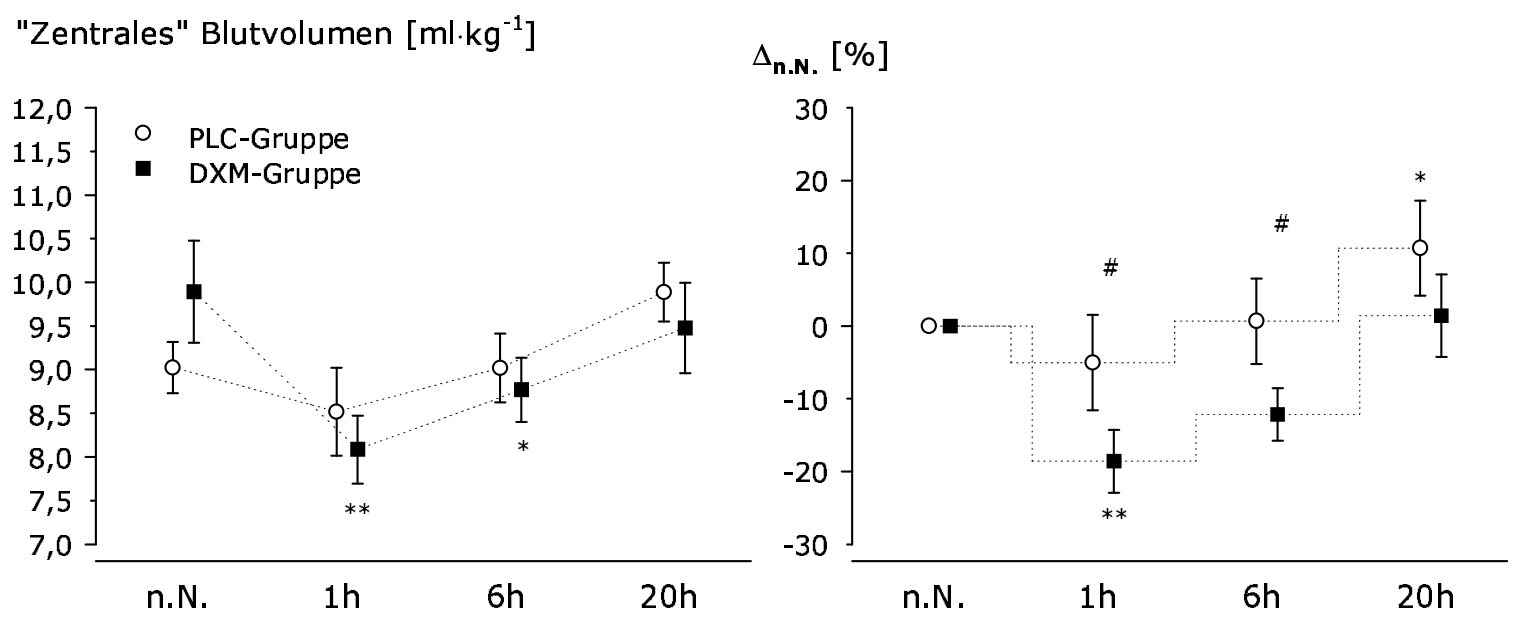

Abbildung 4-1. Zeitlicher Verlauf des ,zentralen“ Blutvolumens ( $\bar{x} \pm$ sem)

PLC $=$ Placebo-Gruppe $(n=10) ;$ DXM = Dexamethason-Gruppe $(n=10)$

n.N. = nach Narkoseeinleitung; 1h, 6h, 20h = eine, sechs, zwanzig Stunden post-OP

$V_{d-\text { zent }}=$,ZZentrales“ Blutvolumen

$\Delta_{n . N .}=$ Relative Änderungen zum Ausgangswert vom ,zentralen“ Blutvolumen

$*(* *) \quad: \mathrm{p}<0,05(0,01): 1 \mathrm{~h}, 6 \mathrm{~h}, 20 \mathrm{~h}$ vs. n.N.

\# $\quad: \mathrm{p}<0,05:$ PLC $v s$. DXM

\begin{tabular}{|c|c|c|c|c|c|}
\hline $\bar{x} \pm \mathrm{sd}$ & & n.N. & $1 \mathrm{~h}$ & $6 \mathrm{~h}$ & $20 \mathrm{~h}$ \\
\hline $\begin{array}{l}V_{\mathrm{d}-z e n t} \\
{\left[\mathrm{ml} \cdot \mathrm{kg}^{-1}\right]}\end{array}$ & $\begin{array}{l}\text { PLC } \\
\text { DXM }\end{array}$ & $\begin{array}{l}9,03 \pm 0,83 \\
9,89 \pm 1,75\end{array}$ & $\begin{array}{l}8,52 \pm 1,43 \\
8,09 \pm 1,24 * *\end{array}$ & $\begin{array}{l}9,02 \pm 1,12 \\
8,77 \pm 1,16 *\end{array}$ & $\begin{array}{l}9,89 \pm 0,95 \\
9,48 \pm 1,46\end{array}$ \\
\hline$\underset{[\%]}{\Delta \mathbf{V}_{\text {d-zent }}}$ & $\begin{array}{l}\text { PLC } \\
\text { DXM }\end{array}$ & & $\begin{aligned} \# & \\
-5,03 & \pm 18,57 \\
-18,58 & \pm 13,00 * *\end{aligned}$ & $\begin{array}{r}\# \\
0,66 \pm 16,53 \\
-12,16 \pm 10,90\end{array}$ & $\begin{array}{c}10,71 \pm 18,52 * \\
1,43 \pm 16,10\end{array}$ \\
\hline
\end{tabular}


Der zeitliche Verlauf des „extravaskulären Lungenwassers“ (EVLW) ist der Abbildung 4-2 und Abbildung 6-8 (S. 77) zu entnehmen. Innerhalb der Placebo-Gruppe blieben die Werte, bis auf einen geringgradigen Anstieg in der frühen postoperativen Phase, über den gesamten Untersuchungszeitraum konstant. Dagegen führte die Vorbehandlung der Patienten mit Dexamethason zu tendenziell niedrigeren postoperativen Werten mit maximaler Senkung des EVLW um etwa $10 \%$ am ersten Tag nach der Myokardrevaskularisation (s. Abbildung 6-8, S. 77). Beim Vergleich des EVLW-Verlaufes zwischen den Gruppen ergab sich ein signifikanter Unterschied 20 Stunden nach Operationsende. Eine (1h) und sechs (6h) Stunden nach der Operation lagen die $p$-Werte der statistischen Auswertung bei 0,079 und 0,07 entsprechend, etwas über die Signifikanzgrenze.

\section{Extravaskuläres Lungenwasser $\left[\mathrm{ml} \cdot \mathrm{kg}^{-1}\right]$}

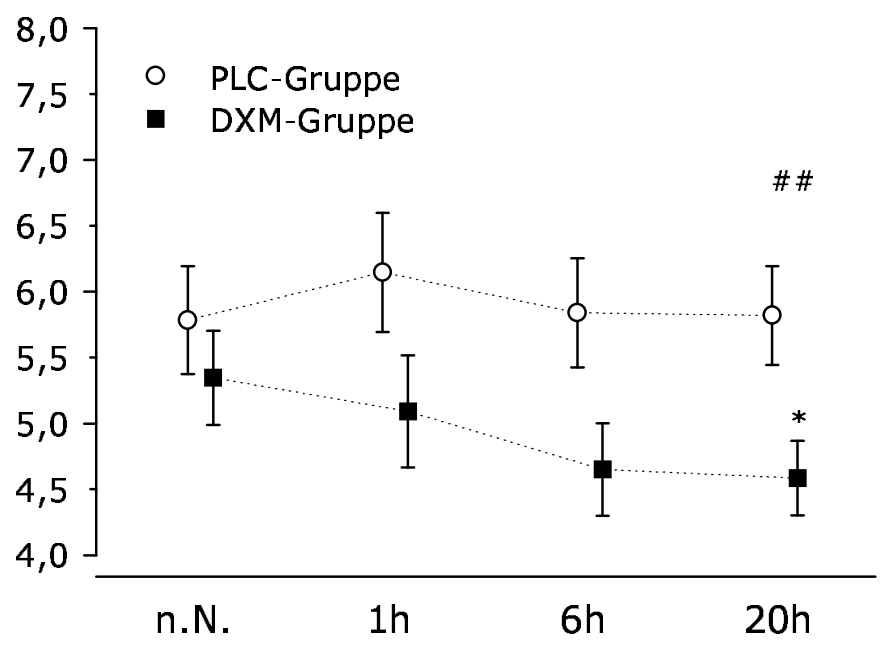

Abbildung 4-2. „Extravaskuläres Lungenwasser“ bei den Patientengruppen ( $\bar{x} \pm$ sem)

PLC $=$ Placebo-Gruppe $(n=10) ;$ DXM = Dexamethason-Gruppe $(n=10)$

n.N. = nach Narkoseeinleitung; $1 \mathrm{~h}, 6 \mathrm{~h}, 20 \mathrm{~h}=$ eine, sechs, zwanzig Stunden post-OP

$E V L W=$,Extravakuläres Lungenwasser“

$*: \mathrm{p}<0,05: 20 \mathrm{~h} v s . \mathrm{n} . \mathrm{N}$.

\#\# : p <0,01: PLC vs. DXM

\begin{tabular}{cccccc}
\hline $\bar{x} \pm \mathrm{sd}$ & & n.N. & 1h & $6 \mathrm{~h}$ & $20 \mathrm{~h}$ \\
\hline & & & & & $\# \#$ \\
$\mathrm{EVLW}$ & PLC & $5,78 \pm 1,00$ & $6,15 \pm 1,28$ & $5,84 \pm 1,17$ & $5,82 \pm 1,06$ \\
{$\left[\mathrm{ml} \cdot \mathrm{kg}^{-1}\right]$} & DXM & $5,35 \pm 1,07$ & $5,09 \pm 1,35$ & $4,65 \pm 1,11$ & $4,58 \pm 0,80^{*}$ \\
\hline
\end{tabular}

\section{6 „Zirkulierendes“ und ,gesamtes“ Blutvolumen}

Für das „,zirkulierende“ und „gesamte“ Blutvolumen bestanden nach Einleitung der Narkose keine signifikanten Unterschiede, obwohl die Placebo-Patienten im Mittel etwas höhere Ausgangswerte aufwiesen (vgl. Abbildung 4-3 und Abbildung 4-4). 
Im zeitlichen Verlauf zeigte das ,zirkulierende“ Blutvolumen $\left(V_{d-z i r k}\right)$ in der DexamethasonGruppe keine wesentlichen Änderungen (vgl. Abbildung 4-3). Demgegenüber lagen die Werte der Patienten in der Placebo-Gruppe von der ersten bis zur sechsten postoperativen Stunde deutlich unter den Ausgangswerten. Betrachtet man die relativen prozentualen Differenzen zum Ausganspunkt, ist in dieser Untersuchungsgruppe ein singnifikanter Abfall um etwa $10 \%$ zum Zeitpunkt $1 h$, folgend von einem deutlich verzögertem Wiederanstieg in den Ausgangsbereich, deutlich zu sehen (vgl. Abbildung 4-3).
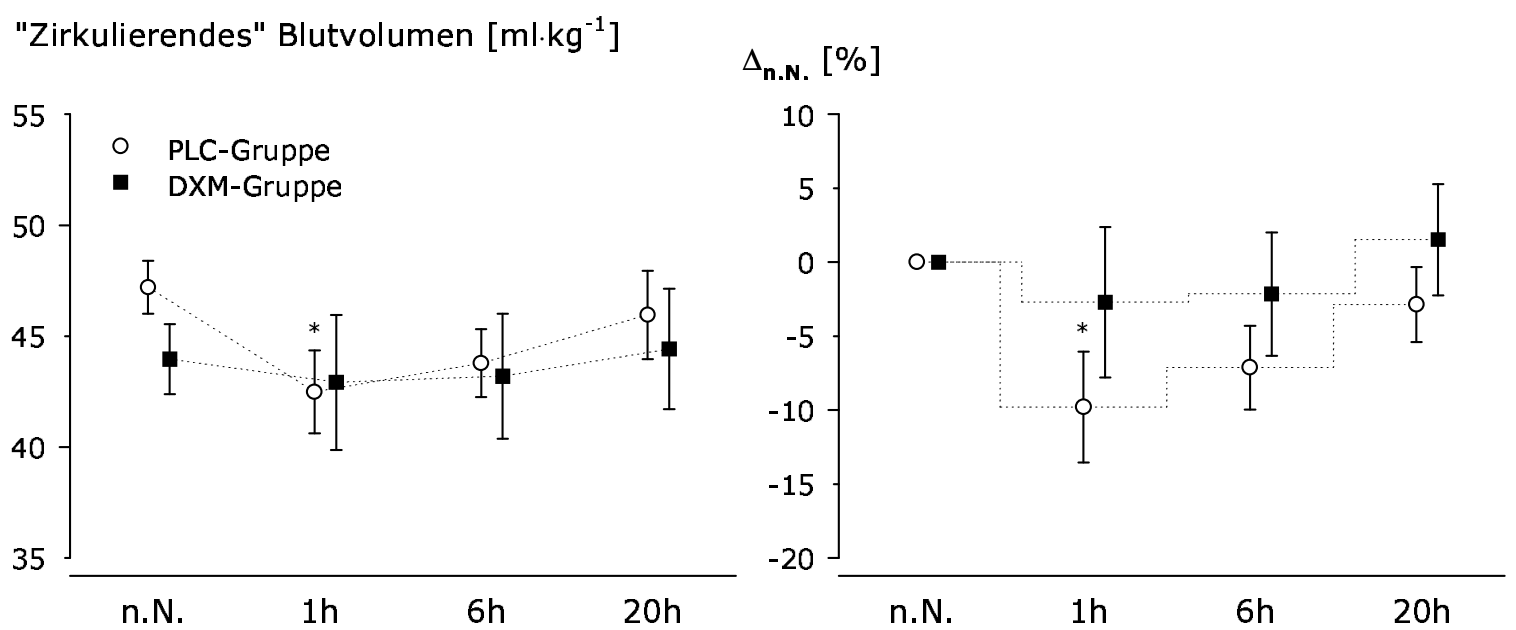

Abbildung 4-3. Zeitlicher Verlauf des ,zirkulierenden“ Blutvolumens ( $\bar{x} \pm$ sem)

PLC $=$ Placebo-Gruppe $(\mathrm{n}=10) ;$ DXM $=$ Dexamethason-Gruppe $(\mathrm{n}=10)$

n.N. = nach Narkoseeinleitung; $1 \mathrm{~h}, 6 \mathrm{~h}, 20 \mathrm{~h}=$ eine, sechs, zwanzig Stunden post-OP

$V_{d-\text { zirk }}=$,Zirkulierendes, , Blutvolumen

$\Delta_{n . N .}=$ Relative Änderungen zum Ausgangswert vom ,zirkulierenden“ Blutvolumen

$*: \mathrm{p}<0,05: 1 \mathrm{~h} v$ s. n.N.

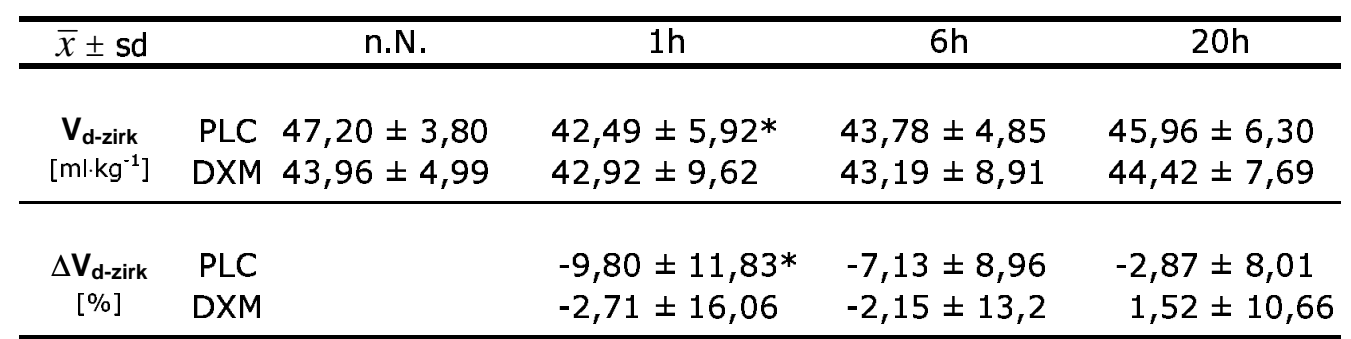

Allerdings konnte im Verlauf des ,gesamten“ Blutvolumens $\left(V_{d \text {-ges }}\right)$ ein anderes Verhalten beobachtet werden ( $v g l$. Abbildung 4-4). Dies fiel bereits eine Stunde nach Operationsende in beiden Gruppen stark ab und verblieb im weiteren Untersuchungszeitraum auf diesem niedrigen Niveau, ohne den Ausgangsbereich wieder zu erreichen. Eine maximale Senkung des $V_{\text {d-ges }}$ um etwa $20 \%$ wiesen die Placebo- und die Dexamethason-Patienten in der späteren postoperativen 
Phase zum Meßzeitpunkt $6 h$ auf. Ein signifikanter Unterschied zwischen den beiden Gruppen konnte statistisch nicht gesichert werden.
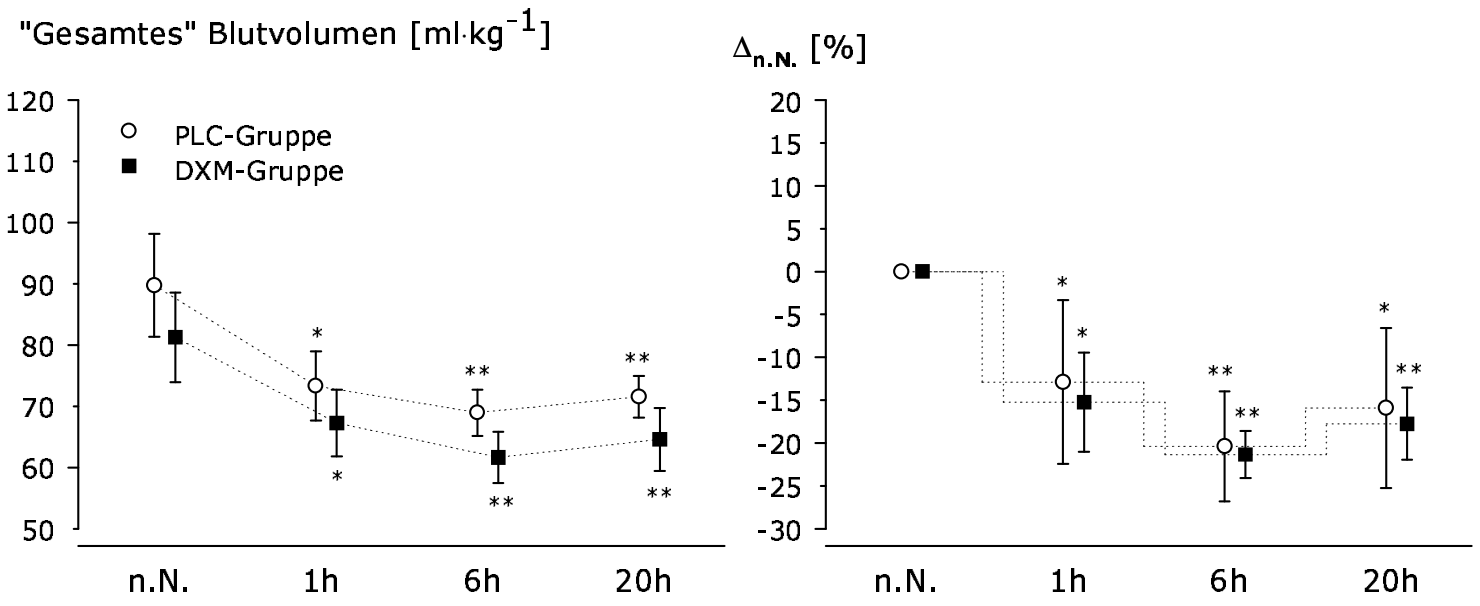

Abbildung 4-4. Zeitlicher Verlauf des ,gesamten“ Blutvolumens ( $\bar{x} \pm$ sem)

PLC $=$ Placebo-Gruppe $(n=10) ;$ DXM = Dexamethason-Gruppe $(n=10)$

n.N. = nach Narkoseeinleitung; $1 \mathrm{~h}, 6 \mathrm{~h}, 20 \mathrm{~h}=$ eine, sechs, zwanzig Stunden post-OP

$V_{d \text {-ges }}=$,"Gesamtes“ Blutvolumen

$\Delta_{n . N .}=$ Relative Änderungen zum Ausgangswert vom ,gesamten“ Blutvolumen

$*(* *): \mathrm{p}<0,05(0,01)$ : 1h, 6h, 20h vs. n.N.

\begin{tabular}{|c|c|c|c|c|c|}
\hline$\overline{\bar{x}} \pm \mathrm{sd}$ & & n.N. & $1 \mathrm{~h}$ & $6 h$ & $20 \mathrm{~h}$ \\
\hline $\begin{array}{l}\text { V }_{\text {d-ges }} \\
{\left[\mathrm{ml} \cdot \mathrm{kg}^{-1}\right]}\end{array}$ & $\begin{array}{l}\text { PLC } \\
\text { DXM }\end{array}$ & $\begin{array}{l}82,91 \pm 21,70 \\
75,61 \pm 22,82\end{array}$ & $\begin{array}{l}69,16 \pm 16,45^{*} \\
63,04 \pm 16,73^{*}\end{array}$ & $\begin{array}{l}65,01 \pm 11,71 * * \\
61,63 \pm 12,60 * *\end{array}$ & $\begin{array}{l}66,80 \pm 11,28 * * \\
64,59 \pm 15,44^{* *}\end{array}$ \\
\hline $\begin{array}{c}\Delta \mathbf{V}_{\mathrm{d}-\mathrm{ges}} \\
{[\%]}\end{array}$ & $\begin{array}{l}\text { PLC } \\
\text { DXM }\end{array}$ & & $\begin{array}{l}-12,90 \pm 27,04^{*} \\
-15,25 \pm 18,27^{*}\end{array}$ & $\begin{array}{l}-20,41 \pm 18,16 * * \\
-21,35 \pm 8,24 * *\end{array}$ & $\begin{array}{l}-15,92 \pm 26,41^{*} \\
-17,76 \pm 12,62^{*} *\end{array}$ \\
\hline
\end{tabular}

\subsection{Verteilung vom Fluß und Volumen im großen Kreislauf}

$\mathrm{Zu}$ den verschiedenen Meßzeitpunkten wurden für jede Gruppe bei Zugrundelegen eines zweikompartimentellen Modells ( $s$. Abschnitt 2.6) die Kreislauf-Transportfunktionen berechnet. Die Parameter der ermittelten Transportfunktionen sind in der Tabelle 8-1 (s. Abschnitt 8.1, S.82f) dargestellt und die sich daraus errechneten Volumina und Flußraten in der Tabelle 4-1 aufgeführt. Die Bedeutung und klinische Relevanz der unterschiedlichen Ausdehnung und Durchblutung der Einzelkompartimente sowie ihr Relation zum ,gesamten“ oder ,peripheren“ Blutvolumen wird unter Abschnitt 5.2 und 6.2.2 diskutiert. 


\begin{tabular}{|c|c|c|c|c|c|}
\hline & & n.N. & $1 \mathrm{~h}$ & $6 \mathrm{~h}$ & $20 \mathrm{~h}$ \\
\hline$V_{d-1 k}$ & PLC & $16,25 \pm 4,11$ & $14,34 \pm 5,22$ & $14,07 \pm 4,65$ & $19,74 \pm 2,32$ \\
\hline$\left[\mathrm{ml} \cdot \mathrm{kg}^{-1}\right]$ & DXM & $15,21 \pm 3,23$ & $15,29 \pm 5,16$ & $13,66 \pm 1,93$ & $18,78 \pm 5,67^{*}$ \\
\hline \multirow{2}{*}{$\underset{\left[l \cdot \mathrm{min}^{-1} \cdot \mathrm{m}^{-2}\right]}{\mathrm{Cl}_{\mathbf{k}}}$} & PLC & $1,35 \pm 0,48$ & $1,54 \pm 0,54$ & $1,56 \pm 0,47$ & $2,08 \pm 0,40 * *$ \\
\hline & $\mathrm{DXM}$ & $1,38 \pm 0,38$ & $1,61 \pm 0,55$ & $1,59 \pm 0,26$ & $2,16 \pm 0,82^{* *}$ \\
\hline \multirow{2}{*}{$\begin{array}{c}\mathbf{V}_{\mathrm{d}-2 \mathbf{2 k}} \\
{\left[\mathrm{ml} \cdot \mathrm{kg}^{-1}\right]}\end{array}$} & PLC & $30,95 \pm 3,70$ & $25,79 \pm 9,92$ & $29,71 \pm 7,29$ & $26,22 \pm 6,44$ \\
\hline & $\mathrm{DXM}$ & $28,75 \pm 3,66$ & $27,62 \pm 7,90$ & $29,53 \pm 7,97$ & $25,64 \pm 7,51$ \\
\hline \multirow{2}{*}{$\underset{\left[1 \cdot \mathrm{min}^{-1} \cdot \mathrm{m}^{-2}\right]}{\mathrm{Cl}_{2 \mathbf{k}}}$} & PLC & $0,97 \pm 0,25$ & $1,30 \pm 1,02$ & $1,58 \pm 0,94 * *$ & $1,00 \pm 0,41$ \\
\hline & $\mathrm{DXM}$ & $0,87 \pm 0,28$ & $1,10 \pm 0,37$ & $1,51 \pm 0,42^{*}$ & $1,07 \pm 0,38$ \\
\hline \multirow{2}{*}{$\begin{array}{c}\mathbf{V}_{\mathbf{d}-\mathbf{p}} \\
{\left[\mathrm{ml} \cdot \mathrm{kg}^{-1}\right]}\end{array}$} & PLC & $36,32 \pm 3,41$ & $31,05 \pm 4,89 *$ & $32,09 \pm 3,60$ & $33,07 \pm 5,10$ \\
\hline & $\mathrm{DXM}$ & $32,40 \pm 4,04$ & $33,15 \pm 9,78$ & $32,95 \pm 8,39$ & $33,23 \pm 7,13$ \\
\hline \multirow{2}{*}{$\begin{array}{c}V_{d-1}(\mathrm{k}) \\
{\left[\mathrm{ml} \cdot \mathrm{kg}^{-1}\right]}\end{array}$} & PLC & $10,00 \pm 2,37$ & $10,69 \pm 7,87$ & $8,50 \pm 3,43$ & $11,48 \pm 1,97$ \\
\hline & $\mathrm{DXM}$ & $8,12 \pm 2,23$ & $9,40 \pm 4,63$ & $8,32 \pm 1,66$ & $11,43 \pm 4,52$ \\
\hline \multirow{2}{*}{$\begin{array}{l}\mathbf{V}_{\mathrm{d}-2 \mathrm{k}(\mathrm{p})} \\
{\left[\mathrm{ml} \cdot \mathrm{kg}^{-1}\right]}\end{array}$} & PLC & $26,32 \pm 3,39$ & $20,35 \pm 7,23^{*}$ & $23,59 \pm 5,05$ & $21,59 \pm 4,99$ \\
\hline & $\mathrm{DXM}$ & $24,28 \pm 2,87$ & $23,75 \pm 7,45$ & $24,63 \pm 7,23$ & $21,80 \pm 6,58$ \\
\hline
\end{tabular}

Tabelle 4-5. Ausdehnung und Durchblutung der von den Kreislauf-Transportfunktionen ermittelten Blutkompartimente und ihre Relation zum ,peripheren“ Blutvolumen ( $\bar{x} \pm \operatorname{sem})$

PLC $=$ Placebo-Gruppe $(\mathrm{n}=10) ;$ DXM $=$ Dexamethason-Gruppe $(\mathrm{n}=10)$

n.N. = nach Narkoseeinleitung; 1h, 6h, 20h = eine, sechs, zwanzig Stunden post-OP

$V_{d-l k, 2 k}=$ Volumen der Einzelkompartimente im Gesamtkreislauf;

$C I_{l k, 2 k}=$ Fluß zu den Einzelkompartimenten;

$V_{d-p}=$,Peripheres zirkulierendes“ Blutvolumen;

$V_{d-l k(p), 2 k(p)}=$ Volumen der Einzelkompartimente im großen Kreislauf;

$*(* *): \mathrm{p}<0,05(0,01): 1 \mathrm{~h}, 6 \mathrm{~h}, 20 \mathrm{~h} v s . \mathrm{n} . \mathrm{N}$.

\subsection{Kumulative Flüssigkeitsbilanzen}

$\mathrm{Zu}$ den verschiedenen Meßzeitpunkten wurden für jede Untersuchungsgruppe die kumulativen Flüssigkeitsbilanzen berechnet ( $s$. Tabelle 4-6). Berücksichtigt wurden sowohl applizierte kristalloide und kolloide Lösungen, als auch Zufuhr und Verlust von Blutkomponenten. Die Bilanzierung der korpuskulären Blutbestandteile spielte eine untergeordnete Rolle, weil sie mengenmäßig keine klinische Relevanz besaß und über die gesamte Meßphase eine ausgeglichene Bilanz zeigte. Um den Einfluß des chirurgischen Eingriffs und der extrakorporalen Zirkulation auf die intraoperative Volumentherapie getrennt betrachtet zu können, wurde ein zusätzlicher Zeitpunkt unmittelbar nach Operationsende $(O p E)$ in Betracht gezogen.

Bei allen Untersuchungspatienten bestand zum Ende der Operation $(O p E)$ eine bedeutende Flüssigkeitsretention, die bis zur sechsten Stunde $(6 h)$ postoperativ weiterhin zunahm. Das während des Eingriffs akkumulierte Volumen wurde erst in der späten postoperativen Phase und 
nur teilweise abgebaut. Es betrug zum Meßzeitpunkt $20 \mathrm{~h}$ noch immer $72,4 \mathrm{ml} \cdot \mathrm{kg}^{-1}$ und $60 \mathrm{ml} \cdot \mathrm{kg}^{-1}$ in der Placebo- und Dexamethason-Gruppe entsprechend. Signifikant unterschieden sich die beiden Gruppen bezüglich der Kristalloiden- und Gesamtbilanz bereits eine Stunde (1h) postoperativ. Das Fehlen von Dexamethason führte in der Placebo-Gruppe zu deutlich positiveren Bilanzen. Diese Differenz blieb weitgehend über den gesamten Untersuchungszeitraum erhalten, nahm allerdings zum letzten Punkt hin etwas ab.

Die Kristalloide- und Kolloide-Nettobilanzen wiesen umgekehrte Verläufe auf. Einerseits nahm das von den applizierten eiweißhaltigen Lösungen beeinflußte kolloidale Volumen zu, andererseits fiel das kristalloide Volumen stetig ab (vgl. Tabelle 4-6). Die Kolloide-Bilanz war bei den Dexamethason-Patienten geringgradig höher, konnten jedoch zwischen den Gruppen keine signifikanten Unterschiede bestätigt werden.

Alle Patienten wurden entsprechend dem intensivmedizinischen Konzept in den ersten 20 Stunden nach der Operation negativ bilanziert. Damit eine ausreichende Diurese gewährleistet wird, wurden bei zurückgehender Ausscheidung (kleiner als $1 \mathrm{ml} \cdot \mathrm{kg}^{-1} \cdot \mathrm{h}^{-1}$ ) Diuretika verabreicht. Bei positiveren Flüssikeits-Gesamtbilanzen wiesen die Placebo-Patienten bereits am Ende der Operation eine signifikant stärkere Diurese gegenüber der Dexamethason-Gruppe (Tabelle 4-6). In der frühen postoperativen Phase blieben die Urin-Stundenportionen in beiden Gruppen gleich, jedoch zeigte sich ab dem Meßzeitpunkt $6 h$ bis 20 Stunden postoperativ erneut eine signifikant stärkere Diurese in der Placebo-Gruppe. Die Diuretikagabe war in beiden Gruppen vergleichbar (vgl. Tabelle 4-4).

\begin{tabular}{cccccc}
\hline & & OpE & $1 \mathrm{~h}$ & $6 \mathrm{~h}$ & $20 \mathrm{~h}$ \\
\hline Kristalloide & PLC & $70,21 \pm 19,03$ & $68,15 \pm 19,14$ & $62,05 \pm 18,60 * *$ & $41,90 \pm 17,47 * *$ \\
{$\left[\mathrm{ml} \cdot \mathrm{kg}^{-1}\right]$} & DXM & $49,78 \pm 13,93$ & $45,24 \pm 15,31$ & $39,50 \pm 14,06 * *$ & $26,75 \pm 9,90 * *$ \\
\hline Erythrozyten & PLC & $0,51 \pm 3,71$ & $1,54 \pm 3,81$ & $0,66 \pm 3,94$ & $1,39 \pm 4,20$ \\
{$\left[\mathrm{ml} \cdot \mathrm{kg}^{-1}\right]$} & DXM & $0,64 \pm 3,65$ & $0,15 \pm 3,50$ & $0,66 \pm 4,16$ & $0,18 \pm 4,54$ \\
\hline & & & & & \\
Kolloide & PLC & $6,39 \pm 8,70$ & $10,86 \pm 11,09$ & $23,41 \pm 13,82 * *$ & $29,10 \pm 20,33 * *$ \\
{$\left[\mathrm{ml} \cdot \mathrm{kg}^{-1}\right]$} & DXM & $9,66 \pm 8,43$ & $16,69 \pm 9,88$ & $27,26 \pm 13,77 * *$ & $33,06 \pm 15,40 * *$ \\
\hline & & $\# \#$ & $\# \#$ & $\# \#$ & $\# \#$ \\
Gesamtbilanz & PLC & $77,11 \pm 25,64$ & $80,55 \pm 28,39$ & $86,12 \pm 31,92$ & $72,39 \pm 30,64$ \\
{$\left[\mathrm{ml} \cdot \mathrm{kg}^{-1}\right]$} & DXM & $60,08 \pm 15,38$ & $62,08 \pm 19,64$ & $67,41 \pm 21,76$ & $59,99 \pm 18,24$ \\
\hline & & & & $\#$ & $\# \#$ \\
Diurese & PLC & $24,99 \pm 20,77$ & $34,33 \pm 20,59$ & $48,53 \pm 23,98$ & $82,72 \pm 31,59$ \\
{$\left[\mathrm{ml} \cdot \mathrm{kg}^{-1}\right]$} & DXM & $16,93 \pm 5,08$ & $25,53 \pm 5,29$ & $37,68 \pm 6,17$ & $60,03 \pm 11,06$ \\
\hline
\end{tabular}

Tabelle 4-6. Kumulative Flüssigkeitsbilanzen der Patientengruppen ( $\bar{x} \pm \mathrm{sd})$

PLC $=$ Placebo-Gruppe $(n=10) ;$ DXM = Dexamethason-Gruppe $(n=10)$

n.N. = nach Narkoseeinleitung; OpE = Operationsende;

$1 \mathrm{~h}, 6 \mathrm{~h}, 20 \mathrm{~h}=$ eine, sechs, zwanzig Stunden post-OP

$*(* *) \quad: \mathrm{p}<0,05(0,01): 1 \mathrm{~h}, 6 \mathrm{~h}, 20 \mathrm{~h} v s . \mathrm{OpE}$

\# (\#\#) : $\mathrm{p}<0,05(0,01):$ PLC vs. DXM 


\section{Diskussion der Methodik}

\subsection{Das mathematische Rezirkulationsmodell}

Die Bestimmung des ,zirkulierenden“ Blutvolumens liegt der Analyse der Indikatorverteilung in einem Transportmedium zugrunde, wobei die erste Verteilungsphase nach der zentralvenösen Farbstoffapplikation entscheidend ist. Das $V_{d-\text { zirk }}$ errechnet sich dann aus der Zeit, die die Indikator-Partikel im Mittel für die Passage des zu messenden Verteilungsvolumens benötigen, wenn das pro Zeiteinheit durchströmende Volumen als Meßgröße vorliegt (Gleichung 2-4, S. 6). Primär markiert also hier der Indikator einen Fluß oder ein „durchflossenes“ Volumen und nicht einen Verteilungsraum wie bei der Messung des „gesamten“ Blutvolumens, die auf dem Prinzip der Massenerhaltung basiert (Gleichung 2-2, S. 5) und eine homogene Verteilung des Indikators zum Zeitpunkt der Messung voraussetzt.

Diesen Gedanken zu Folge muß der initiale Farbstoffbolus nach seiner zentralvenösen Applikation während der Kreislaufpassage einer Umverteilung unterliegen, weil es im zeitlichen Verlauf langsam zu einer homogenen Durchmischung des Indikators im Transportmedium kommt. In diesem Zusammenhang verhält sich der Körperkreislauf ähnlich wie ein elektronischer Filter (Bassingthwaighte 1966b; Bassingthwaighte 1967; Knopp et al. 1976), das heißt der Konzentrations-Zeitverlauf des Indikators am Ausgang des „Kreislauforgans“ stellt eine gedämpfte Abbildung des Eingangssignals dar. Dementsprechend wäre die Ausgangsverteilung ein direktes Abbild der Filtercharakteristika, wenn alle Partikel gleichzeitig den Filter erreichen würden und somit die Eingangsverteilung ein idealer oder Dirac-Impuls wäre ( $\mathrm{gl}$. Abschnitt 2.1.3.2, S. 7). Zur Beschreibung dieser spezifischen Dispersionscharakteristik, der der Indikator beim Durchwandern eines Kreislaufabschnittes unterworfen wurde, bieten sich ähnliche mathematische Methoden an, wie sie im elektrotechnischen Bereich für die Beschreibung von Filtern gebräuchlich sind.

Diese Methoden liegen entsprechenden theoretischen Prinzipien aus der sogenannten „stochastischen Systemanalyse“ zugrunde (Meier und Zierler 1954; Stephenson 1948), wobei charakteristische Eigenschaften eines unbekannten Systems (,black box“) aus der Kenntnis der Eingangs- und Ausgangsfunktion abgeleitet werden können (Bassingthwaighte 1967; Bassingthwaighte und Ackerman 1967). Da aus den Überlegungen im Abschnitt 2.1.3.1 die Stationarität und Linearität des Systems als notwendige Voraussetzungen für die Anwendbarkeit dieses Ansatzes in ausreichendem Maße erfüllt sind, läßt sich der Transportvorgang durch ein Faltungsintegral (Gleichung 2-15, S. 15) beschreiben, welches die Eingangsfunktion mit einer für das System spezifischen Transportfunktion faltet (Lassen und Perl 1979; Bassingthwaighte et al. 1966; Bassingthwaighte und Ackerman 1967; Knopp et al. 1976; Stephenson 1948; Zierler 1962b) und die Impulsantwort des Systems auf den Eingangsimpuls liefert.

Nach der Indikatorapplikation in den rechten Vorhof und seiner vollständigen Durchmischung im rechten Herzen repräsentiert der erste Konzentrationsanstieg der aortal registrierten Dilutionskurve den während der Lungenpassage durch makrodispersive Mechanismen transformierten Farbstoffbolus (,first pass"). Die mit einer zeitlichen Verzögerung folgenden, abgrenzbaren 
Konzentrationsmaxima der Kurve entsprechen dann der Rezirkulation des nicht eliminierten Indikatoranteils aus den einzelnen Organkreisläufen. Die aortale Farbstoffdilutionskurve stellt also die Summe aus zwei Kurvenanteilen dar: aus der Primärkurve (,first pass") und den nach Kreislaufpassage rezirkulierenden nicht eliminierten Farbstoffanteilen.

Da die Primärkurve in ihrem späten Verlauf durch Rezirkulation kontaminiert ist, ist nach Anpassung einer logarithmischen Normalverteilung vom Beginn des Konzentrationsanstiegs bis zu $33 \%$ des Maximums auf dem abfallenden Kurvenanteil der abfallende Schenkel zu extrapolieren. Betrachtet man nun den „first pass“ als Eingangssignal des Kreislaufsystems und den Rezirkulationsanteil als Ausgangssignal nach der Kreislaufpassage, ergibt sich die berechnete aortale Farbstoffverdünnungskurve aus einem sogenannten rekursiven Faltungsalgorithmus (Cutler 1979; van Rossum et al. 1989; Hoeft et al. 1994) als Summe beider simulierter Kurvenanteile, wobei die Ausgangsfunktion dieser Faltung, die das Ergebnis des spezifischen Transportprozesses durch den Körperkreislauf darstellt, wieder als Eingangsfunktion erscheint (s. Abschnitt 2.1.3.2, S. 7)

Zur Lösung nun des inversen Problems und dadurch Ermittlung der Transportfunktion und Berechnung der mittleren Transitzeit des Indikators für die Kreislaufpassage werden durch eine Entfaltungsprozedur (vgl. Abschnitt 2.1.3.3, S. 8) die Parameter einer für die Kreislauf-Transportfunktion geeigneten Modellfunktion schrittweise solange geändert, bis eine optimale Übereinstimmung zwischen berechneten und gemessenen Konzentrations-Zeitverläufen erreicht ist. Dies setzt jedoch voraus, daß sich die gesuchte Transportfunktion durch eine mathematische Modellfunktion beschreibar ist. Diesem einfachen und stabilen numerischen Lösungsweg liegt ein iteratives, nicht-lineares Anpassungsverfahren, welches auf dem Prinzip der kleinsten Abstandsquadrate basiert (Deuflhard und Apostolescu 1980), zugrunde. Die Lösung des Problems wäre theoretisch auch durch eine modellfreie Entfaltung der Dilutionskurven möglich, wobei keine Voraussetzungen über die Grundform der Transportfunktion und der Systemzusammensetzung notwendig sind (Böck et al. 1988). Dieses Verfahren zeigt jedoch ein instabiles numerisches Verhalten (Tikhonov und Arsenin 1977), da die Entfaltung zu einem mit der Zeit zunehmend oszillierenden Verlauf neigt.

Die oben beschriebene Form der Anpassung von experimentellen Transportfunktionen an ein makrodispersives Kreislaufmodell erfordert wegen der vielfach notwendigen Faltungsoperationen einen erheblichen Rechenaufwand. Deshalb sind für die Beschreibung der spezifischen Eigenschaften des Kreislaufsystems nur solche Funktionen geeignet, die mit möglichst wenig Parametern in einer guter Näherung den charakteristischen Verlauf der experimentellen Transportfunktionen beschreiben können. Diese Parameter sollten wenn möglich in direkter Beziehung zu physiologisch relevanten Größen stehen, so daß aus den Parametern Rückschlüsse auf die zugrundeliegenden Mechanismen gezogen werden können. Dementsprechend kommen zum Beispiel asymmetrische Häufigkeitsverteilungen als Kreislauf-Modellfunktionen in Betracht (Bassingthwaighte et al. 1966; Knopp und Bassingthwaighte 1969; Nakai 1981), wobei manchen Autoren eine gute Charakterisierung des Transportvorganges eines Indikators durch den Körperkreilauf mittels linksschiefer Verteilungen, wie die logarithmische Normalverteilung (Böck 1987; Hoeft et al. 1994) oder die ,lagged normal density“ (Bassingthwaighte et al. 1966), gelang. 
In der vorliegenden Studie liegt der Dilutionskurven-Analyse einer logarithmischen Normalverteilung als Modellfunktion für den Kreislauftransport zugrunde (vgl. Abschnitt 2.6.2, S. 17), da dadurch die beste Anpassungsgüte mit einem geringen Rechenaufwand erreicht wurde. Die für die Berechnung des „zirkulierenden“ Blutvolumens benötigte mittlere Transitzeit des Indikators für die Kreislaufpassage ergibt sich dann aus der ermittelten KreislaufTransportfunktion als eine Häufigkeitsverteilung von Transitzeiten (vgl. Abschnitt 2.1.3.2, S. 7).

\subsection{Kompartimentelle Analyse der Farbstoffdilutionskurven}

In der Physiologie unterteilt sich der Blutkreislauf nach funktionellen Merkmalen wie Druck- und Volumenverteilung einerseits in das Niederdrucksystem, das die Funktion des Blutvolumenspeichers erfüllt und ca. $85 \%$ des Blutvolumens enthält und andererseits in das Hochdrucksystem, das der Druckspeicher ist; d.h. es hat ständig Blut hohen Druckes für die Versorgung der Organe bereitzustellen und enthält nur ca. $15 \%$ des Blutvolumens.

Morphologisch besteht der Blutkreislauf des Menschen aus zwei hintereinander geschalteten Hauptabschnitten (Schmidt und Thews 1990, S. 505), dem großen oder Körperkreislauf mit dem linken Ventrikel als Pumpe und dem kleinen oder Lungenkreislauf mit dem rechten Ventrikel als Pumpe, wobei die dem äußeren Gasaustausch dienende Lungenstrombahn eine Sonderstellung einnimmt. Während die übrigen Organstrombahnen alle im großen Kreislauf parallel geschaltet sind, liegt der kleine Kreislauf der Lungenstrombahn in Serie mit dem großen Kreislauf und entspricht dem Gefäßbaum lediglich eines Organs, nämlich der Lunge. Somit muß im Körperkreislauf das Herzzeitvolumen auf alle Organsysteme verteilt werden, die einen unterschiedlich großen Bedarf haben und außerdem in Abhängigkeit vom jeweiligen Aktivitätszustand teilweise stark wechselnde Ansprüche an die Blutversorgung stellen. Demgegenüber passiert den Lungenkreislauf das gesamte Herzzeitvolumen. Die Leber besitzt ebenso eine besondere Stelle im Kreislaufsystem aufgrund ihres arteriellen und venösen Zuflusses. Sie ist demzufolge sowohl parallel als auch seriell zu allen anderen Organen des Splanchnikusgebietes geschaltet.

Bei allen ausgewerteten Farbstoffverdünnungskurven ergaben sich doppelgipfelige KreislaufTransportfunktionen (Abbildung 2-5, S. 18). Tatsächlich wurden sehr gute Übereinstimmungen zwischen berechneten und gemessenen Werten erst nach Zugrundelegen eines zweikompartimentellen Modells (Henthorn et al. 1992; Caldini et al. 1974; Hoeft et al. 1994) und entsprechend Beschreibung der Transportfunktion des Gesamtkreislaufs als Summe von zwei linksschiefen logarithmischen Normalverteilungen erreicht (vgl. Abbildung 2-4, S. 16). Jeder Versuch, das Faltungsprodukt aus Primärkurve (,first pass") und einer einzelnen LognormalVerteilung an den Konzentrations-Zeitverlauf des rezirkulierenden Indikatoranteils anzupassen, führte zu systematischen Abweichungen.

Aus den Ergebnissen der Berechnungen sollte jedoch nicht gefolgert werden, daß sich der Körperkreislauf aus genau zwei Kompartimenten charakterisieren läßt. Tatsächlich läßt sich aber die Makrodispersion des Indikators mit einem rekursiven Faltungsalgorithmus wesentlich besser beschreiben, wenn man für den Kreislauftransport nicht nur ein Kompartiment zugrundelegt. Ob die Annahme eines dritten und weiterer Kompartimente den Transportvorgang des Indikators 
durch den Gesamtkreislauf noch besser zu beschreiben vermag, wurde angesichts der sehr guten Anpassungsergebnisse unter Anwendung des zweikompartimentellen Modells nicht weiter geprüft.

Den Ergebnissen der vorliegenden Studie ist somit zu entnehmen, daß für die Charakteristik der Indikatordispersion während der Kreislaufpassage ein mindestens zweikompartimentelles Modell anzunehmen ist. Dementsprechend lassen sich bezüglich der Verteilung des Blutvolumens und -flusses zwei Körperkompartimente diskriminieren: Das „erste“ oder „schnelle“ Kompartiment mit geringerer Ausdehnung und größeren Stromzeitvolumen. Es repräsentiert jene Organe, die eine hohe Ruhedurchblutung aufweisen (zum Beispiel Herz, Nieren und Gehirn). Demgegenüber das „Zweite“ oder „langsame“ Kompartiment, das vermutlich dem Splanchnikusgebiet zuzuordnen ist (Green 1979), da dieser Kreislaufabschnitt mit Intestinum und Leber zwei seriell geschaltete Organsysteme besitzt.

\subsection{Ermittlung des ,extravaskulären Lungenwassers“}

In der Literatur werden die mittels monoexponentieller Extrapolation aortal registrierter Indikatorverdünnungskurven ermittelten „Normalwerte“ für das „extravaskuläre Lungenwasser“ mit 5-9 ml.kg-1 angegeben (Boldt et al. 1988; Gallagher et al. 1985; Hachenberg et al. 1993; Lumb 1987). Die in der vorliegenden Studie ermittelten Ausgangswerte lagen jedoch mit 5,52 $\pm 1,03 \mathrm{ml} \cdot \mathrm{kg}^{-1}$ deutlich im unteren „Normbereich“.

Die Bestimmung der mittleren Transitzeiten der Indikatoren erfolgt bei der konventionellen Methode durch monoexponentielle Extrapolation der aortalen Indikatordilutionskurven (Boldt et al. 1988; Gallagher et al. 1985; Hachenberg et al. 1993; Lewis et al. 1979; Lewis et al. 1982; Lumb 1987). Dies resultiert jedoch nachweislich eine Überschätzung des $E V L W$, primär aufgrund der Berechnung eines zu großen Verteilungsvolumens des Indikators Kälte (Böck et al. 1987; Hoeft et al. 1991; Mehlhorn et al. 1990; Mihm et al. 1987).

Eine Erklärung hierfür ist auf folgende methodische Fehler zurückzuführen:

1. Wesentliche Ursache ist die Miterfassung eines größeren präpulmonalen Verteilungsvolumens der Kälte durch Austausch des diffusiblen Indikators mit Wandstrukturen des rechten Herzens. Aus den Ergebnissen früherer Untersuchungen mit simultaner Registrierung von Kälte- und Farbstoffkurven in der Arteria pulmonalis ließ sich aus der Transitzeitendifferenz der beiden Indikatoren dieses ,thermoakzessible“ Volumen des rechten Herzens mit $1,36 \pm 0,63 \mathrm{ml} \cdot \mathrm{kg}^{-1}$ berechnen (Mehlhorn et al. 1990). Wenn aber die Überschätzung des $E V L W$ nur dadurch zu erklären wäre, ließe sich dieser additive Fehler mittels einer zusätzlich in der Arteria pulmonalis registrierten Thermodilutionskurve durch das sogenannte „DoppelExtrapolationsverfahren" (Mehlhorn et al. 1990) vermeiden. Bei diesem Verfahren werden die mittleren Organ-Transitzeiten aus der Differenz der extrapolierten aortalen und pulmonalarteriellen mittleren Transitzeiten errechnet.

2. Vielmehr kommt jedoch die Kontamination der aortalen Temperaturkurve durch rezirkulierende Kälte als entscheidende Ursache in Erwägung. Durch die monoexponentielle Extrapolation erfolgt dann nur eine inkomplette Elimination von den Rezirkulationsanteilen 
des Indikators und damit werden zu lange mittlere Transitzeiten ermittelt (Mehlhorn et al. 1990). Noch deutlicher wird dieser Fehler bei pulmonaler Flüssigkeitseinlagerung nach aortokoronarer Bypassoperation und insbesondere im Lungenödem bei ARDS. Durch das langsamere „Auswaschen“ der applizierten Kälte aus der Lunge wird der abfallende Schenkel der Thermodilutionskurve nicht nur durch die erste, sondern eventuell zusätzlich durch die zweite systemische Indikatorrezirkulation kontaminiert.

In der vorliegenden Studie ließen sich die oben beschriebenen additiven Fehler durch zusätzliche Registrierung der Konzentrations-Zeitverläufe für Kälte am Eingang der pulmonalen Strombahn und Verwendung eines Entfaltungsalgorithmus vermeiden. Die mittlere Transitzeit des Indikators erfolgte unabhängig von Injektionsdauer sowie Rezirkulationsphänomena auf der Basis einer pulmonalen Transportfunktion (Bassingthwaighte et al. 1966b; Bassingthwaighte und Ackerman 1967; Knopp et al. 1976; Lassen und Perl 1979; Zierler 1962b). Somit wurde bei der Bestimmung vom EVLW durch die Verwertung der gesamten Information der pulmonal und aortal registrierten Indikatorkinetiken eine höhere Genauigkeit und Sensitivität gegenüber kleinen Veränderungen erreicht (Mehlhorn et al. 1990; Böck et al. 1988).

Die Berechnung des intravasalen Lungenvolumens durch monoexponentielle Extrapolation der aortalen Farbstoffverdünnungskurve scheint die Bestimmung des EVLW nicht entscheidend zu beeinflussen. Die Farbstoffrezirkulation erfolgt erst so spät, daß sie zuverlässig durch monoexponentielle Extrapolation eliminiert wird (Böck 1987; Böck et al. 1988; Mehlhorn et al. 1990).

\subsection{Optimierte Algorithmen zur Bestimmung des ,gesamten“ Blutvolumens}

Bereits im Jahre 1915 versuchten Keith et al. (Keith et al. 1915) Methoden und pharmakokinetische Modelle zu entwickeln, um eine ausreichend korrekte Erfassung der Verteilungsräume intravasal applizierter Indikatoren zu erreichen. In den 30er Jahren wurde der Azofarbstoff Evans blue als Indikator für die Bestimmung der Volumina mit Hilfe der Verdünnungsmethode eingeführt (Gregersen et al. 1935; Gibson und Evans 1937). Die Durchführung jedoch der Bestimmungen war einerseits kompliziert und zeitaufwendig, andererseits könnten die Messungen nicht beliebig oft wiederholt werden, da der Farbstoff sehr langsam aus dem Kreislauf eliminiert wird und daher die „Färbung“ des Plasmas keine weitere Messungen mehr ermöglichte. Eine Alternative zu Evans blue war die Verwendung von radioaktiv markierten Albumin (RIHSA) (Moens et al. 1962), einem Marker des Plasmavolumens, der heute aufgrund seiner Strahlenbelastung und des dadurch limitiert wiederholten Einsatzes als obsolet anzusehen ist.

Die Wichtigkeit jedoch des Kenntnissen des aktuellen Blutvolumens und die nicht ausreichende Ermittlung des Füllungszustandes des kardiovaskulären Systems mittels indirekter hämodynamischer Parameter wie der zentralvenöse Druck $(Z V D)$, der pulmonalkapillärer Verschlußdruck $(P C W P)$ und der systolische oder diastolische systemische arterielle Druck (Hoeft et al. 1994) hatten die Erfindung des Indocyaningrün (ICG) zur Folge. ICG darf aufgrund seiner nicht toxischen Eigenschaften und seiner schnellen Eliminierung beliebig oft in den menschlichen Körper appliziert werden. 
Schad et al. (1987) zeigten eine gute Übereinstimmung der ICG-Plasmavolumina zu den mittels Evans blue berechneten Verteilungsvolumina, obwohl ICG entgegengehalten wurde, daß es wegen seiner schnellen Blutelimination und der dadurch nicht ausreichenden Durchmischung zu dem Zeitpunkt, wo die Elimination anfängt (vgl. Kapitel 5.4.2), nicht für diese Bestimmungen geeignet sei. Heute ist ICG Mittel der Wahl zur Bestimmung von Größe und Änderungen des intravaskulären Volumenstatus (Bradley und Barr 1968; Haneda und Horiuchi 1986; Schad et al. 1987; Haller et al. 1992) und gilt als das konventionelle Referenzverfahren zur Validierung neuer Techniken.

Von Hoeft et al. und Schorn (Hoeft et al. 1994; Hoeft 1995; Schorn 1995) wurden einige Verbesserungsmöglichkeiten beschrieben, auf denen die Bestimmung des Blutvolumens $\left(V_{\text {d-ges }}\right)$ der vorliegenden Untersuchungen basiert. Das modifizierte Verfahren beinhaltet folgende Punkte (vgl. Kapitel 3.4.3):

1. Erneute Kalibrationsprozedur $\mathrm{zu}$ jedem Meßpunkt und für jede einzelne Blutvolumenberechnung

2. Berücksichtigung der Plasmaeigenextinktion und Korrektur der Extinktionsspektren an den Fußpunkten der Kurve bei 600 und 900 nm

3. Registrierung und Auswertung des gesamten Absorptionsspektrum der Blutproben, um das Signal-Rauschen-Verhältnis zu minimieren

4. Iterative Anpassung einer biexponentiellen Modellfunktion an die bis zur 30. Minute erhobenen Werten der Farbstoffkonzentration

5. Rückextrapolation der angepaßten Modellfunktion auf den Zeitpunkt der ersten Leberpassage

6. Korrigieren des Hämatokritwertes für „trapped plasma“ (s. Gleichung 3-26)

\subsubsection{Korrektur der Extinktionsspektren gegenüber dem Leerwertspektrum}

In der Literatur werden verschiedene Methoden beschrieben, die das Absorptionsspektrum der Plasmaproben bezüglich Abweichungen gegenüber dem Leerwertspektrum oder der Plasmahintergrundabsorption jeder Probe, korrigieren (Schad et al. 1987; Nielsen und Nielsen 1962; Nielsen 1963a; Nielsen 1963b). Diese Arbeiten präsentieren sowohl eine Korrektur für die Bestimmung der Plasmakonzentration von Evans blue als auch für Indocyaningrün.

1962 und 1963 schilderten M.H. Nielsen und N.C. Nielsen (Nielsen und Nielsen 1962; Nielsen 1963a; Nielsen 1963b), daß die Eigenextinktion des „ungefärbten“ Plasmas von Blutprobe zu Blutprobe variieren kann, so daß die Eigenabsorption jeder Plasmaprobe berücksichtigt werden muß. Es wurde deshalb versucht, eine rechnerische Beziehung zu entwickeln, die als Basis einer einfachen und präzisen Korrektur der Blutprobenspektren für die Plasmaeigenabsorption dienen sollte. Diese Überlegungen basierten auf den Gesetz von Gaebler (Gaebler 1943), der eine lineare Beziehung zwischen dem negativen Logarithmus der Extinktion einer Plasmaprobe und dem Logarithmus der Wellenlänge für den Spektrumbereich zwischen 600 und $1000 \mathrm{~nm}$ schilderte. Mathematisch kann dies anhand der folgenden Gleichung dargestellt werden: 


\section{Gleichung 5-1}

$$
\begin{aligned}
& -\log E_{\lambda}=b \cdot \log \lambda+a \\
& E_{\lambda} \quad=\text { die Extinktion an der Wellenlänge } \lambda \\
& a, b \quad=\text { Konstanten }
\end{aligned}
$$

Mit anderen Worten ist das Plasma in dieser Spektrumregion als eine „farblose“, nicht lichtabsorbierbare Suspension mit einer gewissen Trübung (blank density) anzusehen (Nielsen und Nielsen 1962). Diese Trübung spiegelt ausschließlich ein von physikalischen Prinzipien dirigiertes scattering-Phänomen nach dem Rayleigh'schen ${ }^{40}$ Gesetz wider. Um die Wirkung des Gaebler'schen Gesetzes auf Basis eigener spektrophotometrischen Messungen nachzuweisen, favorisierten M.H. Nielsen und N.C. Nielsen (Nielsen und Nielsen 1962; Nielsen 1962b) die Arbeitshypothese, daß die Relation zwischen den Logarithmen der optischen Dichte bei zwei definierten Wellenlängen, z.B. 800 und $900 \mathrm{~nm}\left(, E_{800}{ }^{641}\right.$ und , $\left.E_{900}{ }^{“}\right)$, von dem einen zu dem anderen Plasma unabhängig von der Variation der Plasmatrübung konstant ist. Oder anders ausgedrückt besteht eine lineare Beziehung zwischen den negativen Logarithmen bei zwei gegebenen Wellenlängen, so daß die $E_{800}$ immer auf der Basis der Extinktion bei $\lambda=900 \mathrm{~nm}$ kalkuliert werden kann. Nach der Bestimmung der optischen Dichte an diesen zwei Wellenlängen bei 100 humanen farbstoffreien Plasmaproben mit unterschiedlicher Trübung wurde diese Korrelation nachgewiesen. Mittels linearer Regression berechnete sich die folgende mathematische Formel:

\section{Gleichung 5-2}

$$
\begin{aligned}
& -\log E_{800}^{l w}=0,9101 \cdot\left(-\log E_{900}^{l w}\right)-0,033 \\
& E_{\lambda}^{l w} \quad=\text { die Plasma-Eigenextinktion der farbstofffreiein Proben an der Wellenlänge } \lambda
\end{aligned}
$$

Die Konstanten der Gleichung sind von der jeweiligen Konstruktion des Spektrophotometers abhängig. Gemäß Gleichung 5-2 kann jetzt die Plasmaeigenextinktion (bedingt durch die

${ }^{40}$ Die Trübung des Plasmas kann dadurch erkärt werden, daß ein Teil des einfallenden Lichtbündels auf die Plasmateilchen, die gleicher Größenordnung zur Wellenlänge des einfallenden Lichtes sind, gestreut wird. Das Prinzip wird von dem Rayleigh'schen Gesetz beschrieben: $\frac{I_{s}}{I_{0}}=\frac{k}{\lambda^{4}}$, wobei $I_{0}$ und $I_{\mathrm{s}}$ die Intensität des einfallenden und des gestreuten Lichts entsprechend präsentieren, $\lambda$ die Wellenlänge des Lichts darstellt und $k$ ein Faktor ist, der das Volumen der Teilchen in der zweiten Potenz und die Teilchenzahl pro volumetrischer Einheit der Suspension enthält $\left(k=\mathrm{n} \cdot \mathrm{v}^{2}\right)$.

${ }^{41}$ Absorptionsmaximum des Farbstoffs, das mit dem isosbestischen Punkt der Spektralkurven für Oxyhämoglobin und reduziertem Hämoglobin übereinstimmt. Dadurch kann die gemessene Farbstoffkonzentration nicht durch eine Änderung der Sauerstoffsättigung beeinflußt werden. Bei dem verwendeten Farbstoffpräparat der vorliegenden Studie lag das Absorptionsmaximum für ICG nicht bei $800 \mathrm{~nm}$, sondern bei $805 \mathrm{~nm}$. 
kolloidale Trübung des Plasmas) jeder ICG-haltigen Plasmaprobe bei $800 \mathrm{~nm}$ kalkuliert und von der gesamt gemessenen Farbstoffextinktion gleicher Wellenlänge subtrahiert werden.

Ein anderes Verfahren wird von Schad et al. (1987) und Haller et al. (1992) dargestellt, wobei die Messung der Farbstoffkonzentration mit Hilfe eines Dualwellenlänge Densiometers durchgeführt wird. Densiometer arbeiten dadurch, daß eine Wellenlänge $\left(\lambda_{l}\right)$ fixiert ist und die zweite Wellenlänge $\left(\lambda_{2}\right)$ eine Art von relativem Spektrum der Lösung gegenüber dem fixierten $\lambda_{I}$ produziert. Auf dieser Weise wird die Hintergrundabsorption der Probe von dem gesamten Spektrum subtrahiert. Die Autoren benutzten ein Densiometer, das die Extinktion bei $800 \mathrm{~nm}$ relativ zur Extinktion bei $900 \mathrm{~nm}$ zeigte, so daß die Hintergrundabsorption bis zu einem gewissen Grade eliminiert werden konnte.

In der vorliegenden Studie wurde ein rechnerisch aufwendigeres Verfahren eingesetzt, das eine noch bessere Kompensation für die Hintergrundabsorption der Plasmaproben ermöglichte und Signal-Rausch-Verhältnis minimierte. Die im Kapitel 3.4.4 (S. 29) dargestellte Methode besitzt eine höhere Genauigkeit und Sensitivität gegenüber kleinen Veränderungen der Volumenbestimmung, was insbesondere für wissenschaftliche Fragestellungen von Bedeutung ist.

\subsubsection{Kompartimentelle Analyse der Indikatorelimination}

Die Konzentration eines als Bolus applizierten Indikators kann, wie im Kapitel 3.4.4.2 angedeutet wurde, nicht direkt zum Zeitpunkt der Injektion bestimmt werden, weil keine ausreichende Durchmischung mit seinem Verteilungsvolumen existiert. Eine derartige homogene Verteilung der Indikatorpartikel wird erst nach mehreren Kreislaufpassagen erreicht. Da aber während der Zirkulation ein Teil des Indikators eliminiert wird, muß die Konzentration aus seiner Eliminationskinetik auf der Basis eines Eliminationsmodells rückgerechnet werden. Unter vereinfachenden Annahmen und mit Hilfe sogenannter „Kompartiment"-Modelle (Hoeft 1995; Schorn 1995; Schad et al. 1987; Haller et al. 1992; Grainger et al. 1983) kann man diesen zeitlichen Konzentrationsabfall des Indikators oder allgemein die zeitliche Änderungen der Konzentrationen einer verabreichten Substanz in Blut, Plasma oder anderen Körperflüssigkeiten analysieren.

Im einfachsten Fall läßt sich der Konzentrationsverlauf des Indikators unter der Annahme beschreiben, daß er sich in einem einheitlichen Volumen verteilt. Das stellt das einfachste pharmakokinetische Modell dar, bei dem der gesamte Körper als der Verteilungsraum des Indikators, als ein Kompartiment angesehen wird. Dieses Kompartiment kann als „offen“ bezeichnet werden, weil nach Verabreichung der injizierten Menge, die sich unmittelbar im gesamten Kompartiment verteilt, der Indikator daraus eliminiert werden kann.

Für die Beschreibung der Verteilungs- und Eliminationskinetik ist aber bei vielen Indikatoren die Annahme erforderlich, daß zwei oder mehrere Verteilungsräume unterschiedlicher Größe und Zugänglichkeit existieren (entsprechend gibt es Zwei- oder Mehr-Kompartiment-Modelle). Nach diesem Prinzip findet eine Verteilung des Indikators aus einem „,zentralen“ Kompartiment, das den gut durchbluteten Organen entspricht, mit unterschiedlichen Geschwindigkeiten auf ein oder mehrere „periphere“ Kompartimente statt. Die Elimination des Indikators aus diesen 
Kompartimenten läßt sich im allgemeinen wie praktisch jede Form der Elimination durch eine Summe von Exponentialfunktionen beschreiben ( $v g l$. mit Kapitel 3.4.4.2). Grundsätzlich gilt:

\section{Gleichung 5-3}

$$
\begin{array}{ll}
c(t)= & \sum_{i=1}^{n} \alpha_{i} \cdot e^{-k_{i} \cdot t} \text { und } c\left(t_{0}\right)=c_{0}=\sum_{i=1}^{n} \alpha_{i} \\
c(t) & =\text { Die Konzentration des Farbstoffes für den Zeitpunkt } t \\
\alpha_{\imath} & =\text { Gewichtungsfaktor für die Eliminationen des jeweiligen Kompartimentes } \\
k_{i} & =\text { Eliminations-Zeitkonstant jedes Kompartimentes }
\end{array}
$$

Die Beschreibung von komplexen Verteilungs- und Auscheidungsvorgängen mittels eines Kompartiment-Modells stellt jedoch eine erhebliche Vereinfachung der tatsächlich ablaufenden körperlichen Vorgängen dar. Die pharmakokinetischen Kompartimente besitzen demzufolge oft keine physiologische Entsprechung. Viele Autoren (Hoeft 1995; Schorn 1995; Grainger et al. 1983) haben deshalb versucht, reale Organvolumina und Durchblutungsgrößen zugrundezulegen und mittels experimentell ermittelter Daten die komplexen Verteilung- und Eliminationsmöglichkeiten der Indikatoren mit sogenannten „physiologischen“ pharmakokinetischen Modellen darzustellen.

Unter Zugrundelegung eines „physiologischen“ zweikompartimentellen pharmakokinetischen Modells (Hoeft 1995; Schorn 1995; Grainger et al. 1983) erfolgte in der vorliegenden Studie die Auswertung der ICG-Eliminationskinetiken (vgl. Kapitel 3.4.4.2, S. 31). Der Physiologie dieses Modells zu Folge stellen die zwei Kompartimente die hepatische Farbstoff-Elimination aus zwei verschiedenen, unterschiedlich durchbluteten Kreislauf-Kompartimenten nach homogener ICGVerteilung dar.

Entsprechend einem anderen theoretischen Konzept könnte die schnelle Phase des ICG-Abfalls die Aufnahme organischer Anionen des Farbstoffes in die Hepatozyten der Leber und die zweite langsamere Phase das Ausschwemmen des $I C G$ aus der Leber über den zytoplasmatischen Transport und der biliären Ausscheidung (hepatische Exkretion) darstellen (Paumgartner 1975; Kuntz und May 1983; Schorn 1995; Shinohara et al. 1996). Shinohara et al. (1996) konnten in Tierexperimenten zeigen, daß die Behandlung mit Bilirubin, das die Aufnahme von ICG in die Leber kompetitiv hemmt, eine Abnahme der Konstante $\alpha$ der Gleichung 3-24 verursachte und die Gabe von Colchicin, welches toxisch auf die Mikrotubuli wirkt oder von Quabain, das die $\mathrm{Na}^{+}-\mathrm{K}^{+}$-ATPase blockiert, die Konstante $\beta$ erniedrigte. Andere Autoren befürworten die Anwendung komplexerer pharmakokinetischer Modelle wie das Drei-Kompartiment-Modell (Mickulecky 1973) oder das Zwei-Kompartiment-Modell auf der Basis der Michaelis-Menten Eliminationskinetiken (Stoeckel et al. 1980). Diese werden aber nur bei sehr hohen ICG-Dosen, wodurch die Sättigung des hepatischen Eliminationsvorganges erreicht wird, angewendet.

Die ausreichende Präzision der Konzentrationsbestimmung der spät abgenommenen Blutproben ist Vorbedingung zur Erfassung des „langsamen“ exponentiellen Teils (Grainger et al. 1983), der von einem raschen Konzentrationsabfall bis zur ungefähr 15. Minute des Eliminationsvorganges überlagert wird. Schad et al. (1987) zeigten bei Tierexperimenten, daß sich der 
Konzentrationsabfall von $I C G$ während der 5. bis 12. Minute nach Indikatorinjektion durch eine monoexponentielle Funktion beschreiben ließ und daß methodische Voruntersuchungen einen monoexponentiellen Konzentrationsabfall sogar bis zur 30. Minute zeigten. Haller et al. (1992) nutzten ebenso zur Bestimmung von Blutvolumina an Menschen einen monoexponentiellen Abfall bis zur 9. Minute, obwohl sie andeuteten, daß die ICG-Elimination bei allen Probanden ab der 10. Minute einer anderen Kinetik folgte. Die Annahme eines monoexponentiellen ICGAbfalls bis zur 15. Minute hat eine Unterschätzung der berechneten ,virtuellen“ Indikatorkonzentration zum Zeitpunkt der Injektion (vgl. Abbildung 3-7) und dementsprechend eine signifikante Überschätzung der Plasmaverteilungsvolumina der Patienten um etwa $8,133 \mathrm{ml} \cdot \mathrm{kg}^{-1}$ (Student's t-test für unverbundene Stichproben, $p<0,0001$ ) zur Folge. Um die zwei Extrapolationsvorgängen zu vergleichen, wurden bei den Patienten die Plasmavolumina unter Anwendung der beiden Modellfunktionen bestimmt (vgl. Abbildung 5-5).
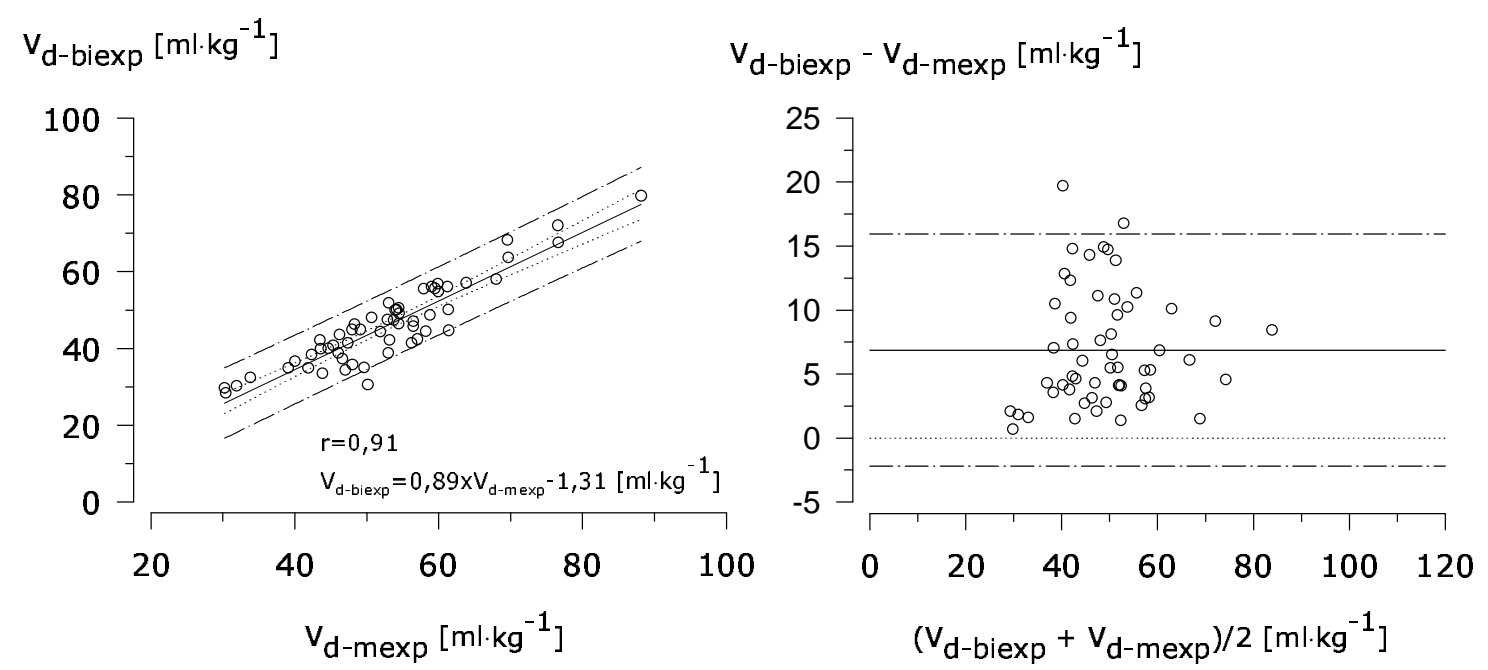

Abbildung 5-5. Vergleich der Methoden zur Ermittlung der Plasmavolumina mittels ein- oder zweikompartimenteller Analyse $(\mathrm{n}=60)$

$V_{d-\text { mexp }}=$ berechnetes Plasmavolumen anhand eines Ein-Kompartiment-Modells (bis 15 min)

$V_{d \text {-biexp }}=$ berechnetes Plasmavolumen anhand eines Zwei-Kompartiment-Modells (bis 30 min)

Viele Autoren verwendeten das Ein-Kompartiment-Modell als die gängige konventionelle Methode zur Bestimmung der Blutvolumina in dem Glauben, daß der produzierte Fehler bei der Vernachlässigung der späteren Kompartimenten sehr gering wäre. Die Unterbewertung dieses Fehlers war wahrscheinlich mit Schwierigkeiten einer präzisen Indikatorbestimmung bei niedrigen ICG-Konzentrationen zusammenverbunden (Grainger et al. 1983). Die Anwendung in dieser Studie einer spektrophotometrischen Analyse anstatt einer monochromatischen Auswertung hatte die Minimierung des produzierten Fehlers aufgrund der Eliminierung des Hintergrundrauschens der Plasmaproben mit niedrigen $I C G$-Konzentrationen zur Folge. 

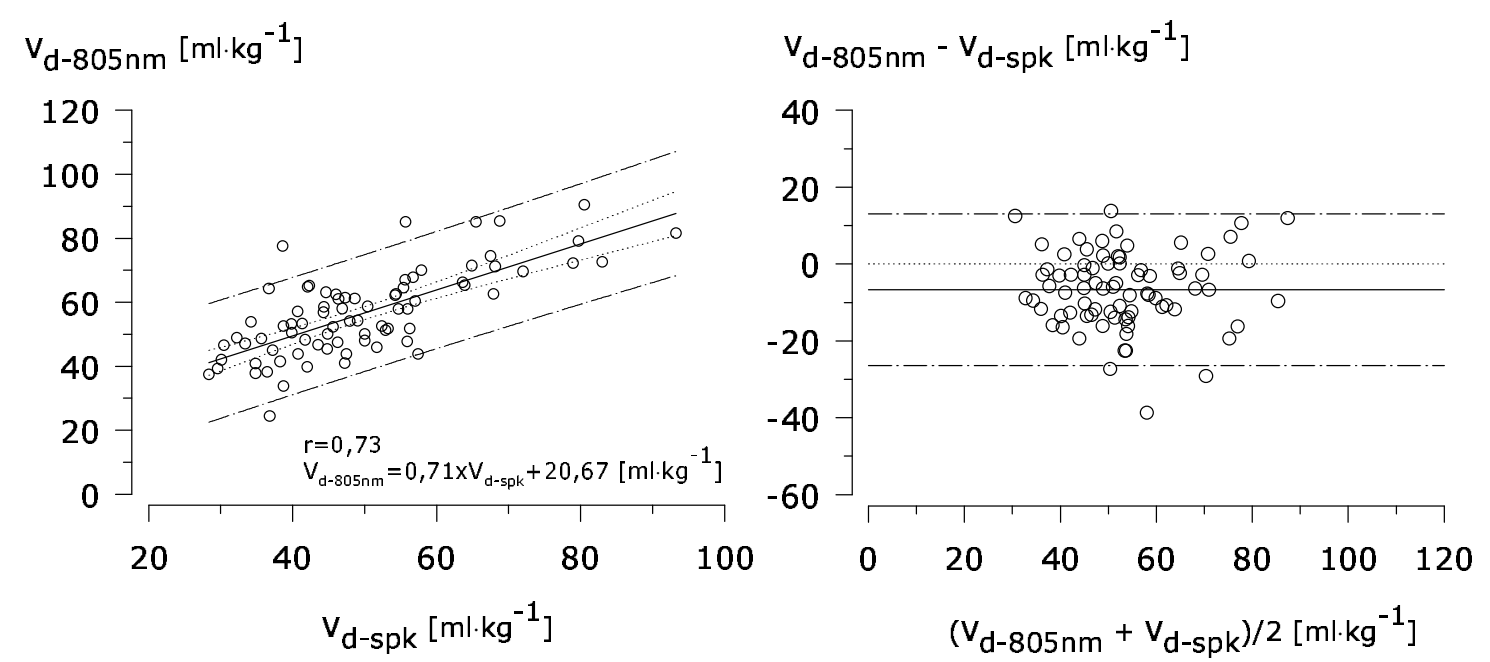

Abbildung 5-6. Vergleich der Methoden zur Ermittlung der Plasmavolumina mittels spektralphotometrischer oder Einwellenlänge-Analyse $(n=80)$

$V_{d-805 \mathrm{~nm}}=$ berechnetes Plasmavolumen mittels Einwellenlänge-Analyse

$V_{d-s p k}=$ berechnetes Plasmavolumen mittels spektralphotometrischer Analyse

Der Abbildung 5-6 ist zu entnehmen, daß eine schwache Korrelation zwischen den berechneten Plasmavolumina besteht. Die Ursache dafür liegt in der Bestimmung der Volumina mit Hilfe der monochromatischen Analyse, wobei bei der Auswertung der einzelnen Patientenkurven eine viel stärkere Streuung der gemessenen Werte um die angepaßte Modellfunktion, im Gegensatz zu den spektralphotometrischen Messungen, besonders bei niedrigen Konzentrationen zu erkennen war. Das nicht optimierte Signal-Rausch-Verhältnis, verursacht durch die mangelnde Elimination der nicht vom Indikator bedingte Hintergrundabsorption der Plasmaproben, hatte eine Instabilität der Reproduzierbarkeit der Messungen und eine sogar signifikante Überschätzung ${ }^{42}$ der Plasmavolumina um etwa $6,342 \mathrm{ml} \cdot \mathrm{kg}^{-1}$ zur Folge (Student's t-test für unverbundene Stichproben, $p=0,02)$.

\subsubsection{Rückextrapolation auf dem Zeitpunkt der ersten Leberpassage}

Chiou (1979) vertritt die Meinung, daß die konventionelle Rückextrapolation der angepaßten Modellfunktion auf den Zeitpunkt der Indikatorinjektion und die Berechnung der Indikatorkonzentration zu diesem Zeitpunkt streng gesehen nicht korrekt ist. Bei der Anwendung des klassischen pharmakokinetischen Modells, wobei die Konzentration $c_{0}$ als die Summe der Exponentialkoeffizienten (vgl. Gleichung 5-3) kalkuliert wird, wird die Existenz einer Latenzzeit bis zum eigentlichen Beginn der Indikatorelimination nicht berücksichtigt. Um diesen Zeitpunkt der ersten Leberpassage abzuschätzen, notierten Haneda und Horiuchi (1986) und Haller et al. (1992) den Erscheinungszeitpunkt des Farbstoffes in der peripheren Zirkulation mittels eines

${ }^{42}$ Schorn (1995) zeigte bei seinen Untersuchungen, daß die berechneten Bluvolumina unter Anwendung der beiden Auswertungsmethoden im Mittel eine gute Übereinstimmung aufwiesen. Die Differenzen der Einzelwerte jedoch, die als Maß für die Zuverlässigkeit der einen oder anderen Methode dienten, zeigten bei diesen Messungen eine erhebliche Streuung auf. 
Ohrdensiometers. Unter der Voraussetzung, daß dieser Zeitpunkt auch der Zeitpunkt der ersten Leberpassage sei, kalkulierten sie die Blutvolumina durch Rückextrapolation auf diesen Erscheinungspunkt. Sie schilderten im Mittel 4,8\% (Haneda und Horiuchi 1986) und $5 \%$ (Haller et al. 1992) höhere Werte für das Plasmavolumen. Bei den vorliegenden Untersuchungen wurde als Zeit der ersten Leberpassage die halbe mittlere Kreislaufzeit (vgl. Kapitel 3.4.4.2) verwendet. Die errechneten Volumina werden in Abbildung 5-7 dargestellt.
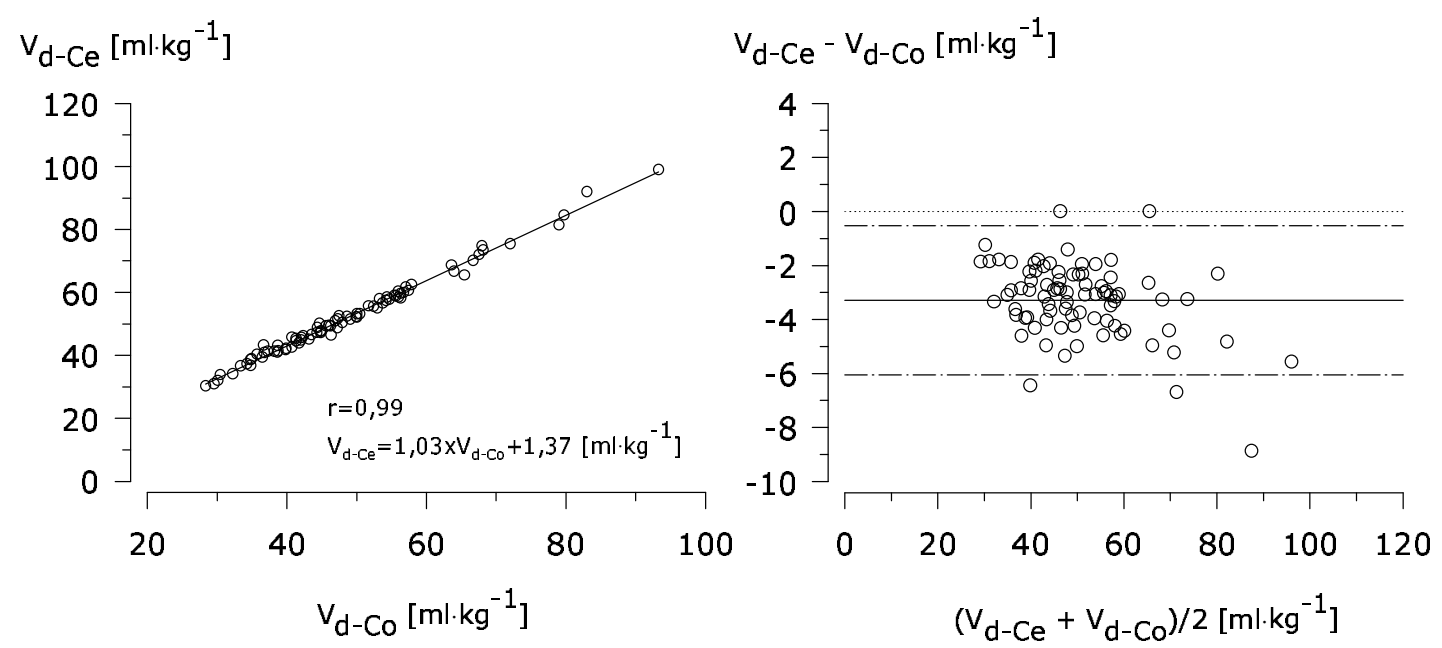

Abbildung 5-7. Vergleich der Methoden zur Ermittlung der Plasmavolumina mittels Rückextrapolation auf den Nullzeitpunkt oder auf den Zeitpunkt der ersten Leberpassage $(n=82)$

$V_{d-C o}=$ berechnetes Plasmavolumen mittels Rückextrapolation auf dem Nullzeitpunkt

$V_{d-C e}=$ berechnetes Plasmavolumen mittels Rückextrapolation auf dem Zeitpunkt der ersten

Leberpassage

Es wurden entsprechend um etwa $2,427 \mathrm{ml} \cdot \mathrm{kg}^{-1}$ höhere Werte für das Plasmavolumen (das entspricht eine Differenz um 4,87 \%) unter Rückextrapolation auf den Zeitpunkt der tatsächlichen Indikatorelimination berechnet. Die halbe mittlere Kreislaufzeit betrug für die Messungen nach Einleitung der Narkose der Patienten als Ausgangszeitpunkt 25,67 $\pm 5,59 \mathrm{sec}^{43}$. Diese ist höher als die aufgezeichneten Werte von Haller et al. (1992) für den Erscheinungspunkt des ICG in der Peripherie. Diese betrugen zwischen 12 und 17 Sekunden als Zeitpunkt der ersten Leberpassage.

\subsubsection{Validierung der erhobenen Daten für das ,gesamte“ Blutvolumen}

Das Verfahren der Rückextrapolation zur Berechnung von Verteilungsvolumina wird von manchen Autoren in Frage gestellt. Eine Rückextrapolation auf den Zeitpunkt der Indikatorapplikation wäre theoretisch nur dann gerechtfertigt, wenn ein ideales System mit kompletter

\footnotetext{
${ }^{43}$ Im Rahmen anderer Untersuchungen des gleichen Projektes betrug die halbe mittlere Kreislaufzeit des Farbstoffindikators vor Einleitung der Narkose 20,55 4,44 sec. Der Einfluß der Narkose und die Volumengabe während der Einleitung haben eine Zunahme der Kreislaufzeiten zur Folge (vgl. Kapitel $8.2)$.
} 
instantaner Durchmischung des Indikators zum Zeitpunkt der Applikation vorliegen würde. Bei der Rückextrapolation wird also eine „,virtuelle“ Konzentration berechnet, die man erhalten hätte, wenn sich der Indikator instant in seinem Verteilungsvolumen durchgemischt wäre. Dies ist jedoch bei rein intravasalen Indikatoren nicht der Fall, denn es müssen mehrere Kreislaufpassagen abgewartet werden, bevor eine gleichmäßige Durchmischung stattgefunden hat. Trotzdem das Rückextrapolationsverfahren aufgrund dieser theoretischen Schwierigkeiten von manchen Autoren sogar abgelehnt wird, sprechen eine Vielzahl von empirischen Untersuchungen vor allem im Bereich der Pharmakokinetik dafür, daß dieses Bestimmungsverfahren zumindest für eine abschätzende Messung eines Verteilungsvolumens geeignet ist (obwohl diesbezüglich kein konkreter theoretischer Hintergrund existiert).

Die bei den kardiochirurgischen Patienten errechneten Plasma- und Blutvolumina nach der Narkoseeinleitung (vgl. Abbildung 4-4) sind zwar etwas größer, aber mit den in der Literatur beschriebenen vergleichbar (Haller et al. 1992; Hurley 1975; Hoeft et al. 1994; Schorn 1995). Im Vergleich zu den Kapiteln 5.4.2 und 5.4.3 wäre es im Grunde genommen zu erwarten, daß nach Anwendung einer biexponentiellen Funktion und einer spektralphotometrischen Auswertung die Volumina niedriger als die in der Literatur mittels der konventionellen Methode errechneten berechnet werden. Die Einleitung einer Narkose mit Sufentanil ist aber häufig mit einem Abfall des Blutdrucks vergesellschaftet, dem in der Regel mit der Gabe von Volumen begegnet wird (vgl. Kapitel 8.2). Dementsprechend ist eine Zunahme des aktuellen Blutvolumens nach Narkoseeinleitung zu verzeichnen.

Die ermittelten Volumina zeigten eine gute Übereinstimmung mit den von Schorn (1995) veröffentlichten Daten. Diese wurden aus einem vergleichbaren Patientenkollektiv und unter Anwendung der gleichen Auswertungsmethoden erhoben. 


\section{Diskussion der klinischen Ergebnisse}

\subsection{Kausale Pathogenese des „systemic inflammatory response syndrome“}

Seit der ersten Operation am offenen Herzen in den fünfziger Jahren wird der Einfluß der extrakorporalen Zirkulation vor allem im Rahmen koronarchirurgischer Eingriffe beobachtet und untersucht. Chenoweth et al. (1981) und Kirklin et al. (1983) versuchten in den achtziger Jahren mittels einiger meßbarer Parameter der humoralen und zellulären Aktivierung, die postoperative pulmonale Dysfunktion zu erforschen. In den heutigen Jahren ist bekannt, daß im Rahmen einer Herzoperation durch die Hämodilution mit dem Füllvolumen der Herz-Lungen-Maschine (Hoeft et al. 1991; Schorn 1995), durch die myokardiale Ischämie (Gebhard 1990) sowie durch den Blutkontakt mit Fremdoberflächen (Chenoweth et al. 1981; Kirklin et al. 1983; Boldt et al. 1986; Westaby 1987; Haeffner-Cavaillon et al. 1989; Jansen et al. 1991a; Jansen et al. 1991b; Schorn et al. 1992; Butler et al. 1993; Elliott und Finn 1993; Schorn 1995) verschiedene inflammatorische Mediatorsysteme aktiviert werden. Diese können zu kritischen Mikrozirkulationsstörungen und zu einer Freisetzung vasoaktiver Substanzen sowie permeabilitätsaktiver Stoffwechselprodukte führen. Dieser Prozeß, der in seinem Ablauf mit den Folgen eines schweren Traumas, einer Sepsis oder eines Schocks vergleichbar ist, führt $\mathrm{zu}$ einer Schädigung des besonders empfindlichen Kapillarendothels mit konsekutiver Steigerung der Membranpermeabilität und anschließend zu einem Ungleichgewicht der die Flüssigkeitsverteilung zwischen Intra- und Extravaskulärraum regulierenden Starling'schen Kräfte. Diese unspezifischen humoral und zellulär systemischen Reaktionen, die sich unter dem Einsatz der extrakorporalen Zirkulation manifestieren, werden unter dem Begriff „Postperfusions“- oder „post-pump“-Syndrom zusammengefaßt. Ein derartiges generalisiertes Inflammationsgesschehen (,systemic inflammatory response syndrome“, SIRS), bei dem nach Butler et al. die Wichtigkeit der ,akuten Phase-Reaktion“ zu dem chirurgischen Trauma nicht zu unterschätzen ist (Butler et al. 1993), kann eine Funktionsstörung verschiedener Organsysteme verursachen. Diese äußert sich insbesondere als eine pulmonale, kardiale, renale Dysfunktion oder eine erhöhte postoperative Blutungsneigung (Chenoweth et al. 1981; Kirklin et al. 1983; Westaby 1987; Butler et al. 1993).

\subsection{1 Ätiologisch wirksame inflammatorische Faktoren}

Die genaue kausale Pathogenese einer nach extrakorporaler Zirkulation auftretenden Leukozytose, der durch die erhöhte Kapillarpermeabilität bedingte interstitielle Flüssigkeitsakkumulation und einer postoperativ eventuell auftretenden Organsystem-Dysfunktion ist bis heute ungeklärt. Nach der Erstbeschreibung durch Dodrill (1958) beschreibt Weyland (1984) eine Vielzahl ätiologisch wirksamer primären und sekundären Ursachen, die zur Entstehung der Komplikationen der offenen Herzchirurgie beitragen. In den folgenden Abschnitten sollen die Faktoren der humoral und zellulär bedingten unspezifisichen generalisierten Entzündungsreaktion beschrieben werden, die wichtige Determinaten einer möglichen Organdysfunktion darstellen (Chenoweth et al. 1981; Kirklin et al. 1983; Westaby 1987; Butler et al. 1993; Elliott und Finn 1993). 


\subsubsection{Aktivierung des Komplementsystems}

Chenoweth et al. (1981) und Kirklin et al. (1983) beschreiben, daß der Blutkontakt mit Fremdoberflächen unter Einsatz der extrakorporalen Zirkulation Veränderungen der Plasmaproteine, insbesondere der des Gerinnungssystems, verursacht. Die unspezifische Aktivierung des Komplementsystems überwiegend über den alternativen Weg (Schmidt und Thews 1990, S. 453; Butler et al. 1993) führt zur Bildung der Anaphylatoxine C3a und C5a. Obwohl allein aufgrund des operativen Traumas eine derartige Aktivierung des Komplementsystems gerechtfertigt wäre, zeigten Kirklin et al. (1983), Butler et al. (1993) und Jansen et al. (1991a), daß C3a rasch mit dem Beginn des kardiopulmonalen Bypasses anstieg und höchste Plasmaspiegel am Ende der extrakorporalen Zirkulation erreicht wurden. Diese These wird durch die Tatsache gestützt, daß die C3a-Werte eine gute Korrelation zur Dauer des CPB zeigten und daß der C3a-Anstieg bei „geschlossenen“ herzchirurgischen Eingriffen nicht nachweisbar war (Kirklin et al. 1983). Die alleinige Aktivierung des Komplementsystems ist nicht ausreichend, um eine kritische respiratorische Dysfunktion, eine renale Insuffizienz oder Gerinnungsstörungen zu induzieren. Jedoch führt die Expression kleiner Mengen von C5 zusätzlich zur Aggregation und Margination von Leukozyten. Durch die Bindung des C5a an spezifische Membranrezeptoren der Neutrophilen wird die Adhäsion der Leukozyten an intravaskulären Endothelzellen erleichtert. Dies stellt ein sehr wichtiges Ereignis während der Reperfusionsphase der Lungen dar.

Nach Schmidt und Thews (1990) sind bei der Komplementaktivierung über den alternativen Weg nicht nur die kleinen und hauptsächlich chemotaktisch und permeabilitätssteigernd wirkenden Bruchstücke der Komplementfaktoren von Bedeutung, sondern auch die größeren Peptidbruchstücke wie C3b und inaktives C3b. Diese binden sich an die Materialien der HLM und induzieren auf diese Weise die Degranulierung der neutrophilen Granulozyten, die Zytokinfreisetzung von den Monozyten (Gu et al. 1993) sowie die Aktivierung weiterer Komplementfaktoren (Butler et al. 1993; Westaby 1987; Jansen et al. 1991a). Schließlich wird der zytolytische Komplex C5b-9 gebildet, der die Leukozytenaktivierung über Steigerung des intrazellulären ArachidonsäureMetabolismus verstärkt.

\subsubsection{Blutgerinnung und Fibrinolyse}

Der erste Kontakt des Blutes mit den negativ beladenen Fremdoberflächen der HLM und das Kollagen, das durch die Gewebeverletzung entsteht, aktivieren nicht nur das Komplementsystem sondern auch den Gerinnungsfaktor XII. Der aktivierte Faktor XIIa stimuliert die Leukozytenaggregation und die Freisetzung von Elastase (Butler et al. 1993), die die Kallikrein-Produktion und durch positives feedback wiederum die Aktivierung des Faktors XII induziert. Kallikrein selber stimuliert die Granulozytenchemotaxis, -aggregation und -degranulierung. Gleichzeitig wird dadurch das Kinin-Bradykinin-System aktiviert, welches wahrscheinlich eine Vasodilatation und Permeabilitätssteigerung bewirkt. Diese Effekte in Kombination mit einer Steigerung der Fibrinolyse und einer Störung der Fibrinbildung durch die vermehrt entstandenen Fibrinspaltprodukte können eine progrediente Zerstörung der Kapillarwandintegrität, eine Transsudation von Flüssigkeit ins Interstitium (,capillary leakage“) und eine Störung der normalen Hämostasis verursachen (Westaby et al. 1987). 


\subsubsection{Verhalten der Leukozyten und Thrombozyten}

Initial nach Anschluß der HLM wird von vielen Autoren (Kirklin et al. 1983; Boldt et al. 1986; Westaby 1987; Jansen et al. 1991a; Butler et al. 1993) ein Abfall der Leukozytenzahl beschrieben, der wahrscheinlich durch die Hämodilution mit dem Füllvolumen der HLM oder durch die Adhäsion der Granulozyten an die Kunstoberflächen hervorgerufen wird. Eine sich anschließende Leukozytose kommt durch die Mobilisierung der marginal ${ }^{44}$ befindlichen Zellen und durch eine erneute Freisetzung von unreifen weißen Blutkörperchen aus dem Knochenmark zustande. Während die Lungendurchblutung unter dem CPB sistiert, bleibt nach Westaby (1987) ein Teil der Granulozyten und Lymphozyten in der Lunge. Nach Entfernung der Aortenklemme verharren dann diese Granulozyten (jedoch nicht die Lymphozyten ${ }^{45}$ ) in den Lungenkapillaren (Butler et al. 1993). Diese sequestrierten Granulozyten könnten inaktiv und unschädlich sein. Manche Autoren vermuten aber, daß die Freisetzung von Lipid-Peroxidase-Produkten zu Verletzungen der Zellmembranen führt, zur gleichen Zeit wie die pulmonale Granulozytensequestrierung nach Entfernung der Aortenklemme stattfindet (Glauser und Fairman 1985; Butler et al. 1993; Westaby 1987). Die Freisetzung von granulozytenspezifischer Elastase (in den primären azurophilen Granula enthalten) und verschiedenen Glycoproteinen wie Lactoferrin (sekundäre Granula) führt auch zu Kapillarschäden in der Lungenstrombahn, da die Wirkung der gewebeprotektiven Antiproteasen durch die erhöhte Bildung von Sauerstoffradikalen geschwächt wird.

Die Blutplättchen haften ebenfalls an den Lungenkapillaren und verstärken durch die Serotoninproduktion die Adhäsion der Granulozyten an den Endothelzellen (Westaby 1987).

\subsubsection{Die Rolle der Endotoxinfreisetzung}

Jansen und Mitarbeiter (1992) beschreiben, daß tatsächlich Endotoxin durch die applizierten Infusionen und durch die mit Endotoxin kontaminierten Schläuche der HLM zu Beginn der extrakorporalen Zirkulation freigegeben wird. Dieses „environmental“ Endotoxin ist im Gegensatz zu dem durch gram-negative Keime freigesetzten Endotoxin nicht pathogen (Jansen et al. 1992). Zusätzlich wird aber aufgrund des reduzierten mittleren arteriellen Drucks während des CPB eine Hypoperfusion des Splanchnikusgebietes mit konsekutiver Schädigung der MukosaBarriere zwischen Darm- und Kapillarwänden hervorgerufen. Hierdurch kann ein Zufluß von großen Endotoxinmengen in die Portalvene und eine Translokation der Darmbakterien induziert werden. Dementsprechend durchdringen Bakterien, die normalerweise auf das Darmlumen beschränkt sind, die intestinale Mukosa-Barriere und erscheinen in den mesenterialen Lymphknoten oder in anderen organischen Systemen (Jansen et al. 1992; Butler et al. 1993). Unter normalen Bedingungen wirken Kuppfer'sche Zellen der Leber einer systemischen Endotoxämie durch Endotoxinelimination entgegen. Jedoch ist unter Einfluß der EKZ das retikuloendotheliale System supprimiert.

\footnotetext{
${ }^{44}$ Etwa 50\% der neutrophilen Granulozyten im intravasalen Raum zirkulieren nicht, sondern haften an der Endothelwand insbesondere der Lungen- und Milzgefäße (Schmidt und Thews 1990, Seite 435).

${ }^{45}$ Westaby (1987) beschreibt als Grund dafür, daß im Gegensatz zu den Lymphozyten die polymorphkernigen Granulozyten spezielle Membranbindungsstellen für C5a-Anaphylatoxin besitzen.
} 
Die Endotoxinfreisetzung in die systemische Zirkulation führt zur Aktivierung des Gerinnungssystems und der Granulozyten. Es kommt zur Verstärkung der Adhäsionsbereitschaft der Endothelzellen und zur Produktion ${ }^{46}$ von Entzündungsmediatoren wie tumor necrosis factor$\alpha(T N F-\alpha)$, Interleukin-1 $\beta$ und Interleukin-6 (Jansen et al. 1992; Butler et al. 1993). TNF- $\alpha$ wird als einer der wichtigsten Faktoren im Hinblick auf die Pathophysiologie der gram-negativen Sepsis und der postoperativen Multiorgan-Dysfunktion angesehen (Jansen et al. 1991a; Jansen et al. 1992; Butler et al. 1993; Dauber et al. 1993).

\subsubsection{Humorale Zytokinproduktion und die Rolle der Adhäsionsmoleküle}

Viele Autoren vertreten die Meinung, daß die primäre inflammatorische Antwort zum CPB durch die Aktivierung des Komplementsystems und die Produktion von Anaphylatoxinen bedingt ist. Jedoch scheint die spätere postoperative Phase durch die Freisetzung von Zytokinen aus den aktivierten polymorph- und monokernigen Leukozyten, den Lymphozyten und den Endothelzellen geprägt zu sein. (Butler et al. 1993; Butler et al. 1992; Haeffner-Cavaillon et al. 1989; Deng et al. 1995; Menasché et al. 1994; Kalfin et al. 1993; Finn et al. 1993; Elliott und Finn 1993).

Interleukin-1 (IL-1), das ein starkes endogenes Pyrogen darstellt, bewirkt zum Beispiel während der frühen und späten postoperativen Phase eine Komplementaktivierung und eine Endotoxinfreisetzung. Es verursacht Leukozytose und Fieber, eine verstärkte C-Reaktivprotein- (HaeffnerCavaillon et al. 1989) und IL-6- Freisetzung. IL-6 induziert in der späteren Phase die Produktion von Akute-Phase-Proteinen und die Freisetzung von Adrenokortikalhormonen (Butler et al. 1993). Ebenfalls in der späten Phase führt IL-8 unter Einfluß der Komplementtoxine zu einer gesteigerten Synthese von Sauerstoffradikalen und Aktivierung der Leukozyten.

Eine derartige Aktivierung der Leukozyten und ihre Interaktion mit dem Endothel könnte als wichtige Determinaten der endothelialen Funktionsstörung betrachtet werden (Boldt et al. 1995). Während der ersten Phase der Adhäsion ${ }^{47}$ (,primäre Adhäsion“) gehen die Leukozyten von der axialen zur peripheren Strömung über und „rollen“ anschließend mit niedriger Geschwindigkeit entlang der Gefäßwände (Elliott und Finn 1993). Dieses „Leukozyten-rolling“ ist eine Folge der Interaktionen der neu exprimierten endothelialen Selektine mit den entsprechenden Liganden auf der Granulozytenoberfläche. Nach einer zweiten Phase, währenddessen chemotaktische Substanzen sowohl von den Leukozyten als auch von den Endothelzellen freigesetzt werden, folgt die dritte Phase der Adhäsion (,sekundäre Adhäsion“) mit Migration der Leukozyten durch die tight-junctions der Endothelzellen in das perivaskuläre Gewebe.

\footnotetext{
${ }^{46}$ Endotoxin, das Lipopolysacchariden (LPS) entspricht, bildet ein Komplex mit dem Lipopolysacchariden-bindendes Protein $(L B S)$, welches dann an die Leukozyten-CD14-Rezeptoren bindet und die Zytokin-Freisetzung bewirkt.

${ }^{47}$ Es gibt drei bekannte Rezeptorenfamilien, die für die Adhäsion von Bedeutung sind: Die Immunglobulin-Superfamilie (z.B. ICAM-1, VCAM-1), die Integrin-Familie und die Selektine [z.B. ELAM-1, L-selectin-leukocyte endothelial cell adhesion molecule (LECAM), P-selectin-granule membrane protein 140 (GMP140)] (Boldt et al. 1995).
} 
Andere inflammatorische Mediatoren wie TNF- $\alpha$, LPS und IL-8 bewirken ebenso Änderungen der endothelialen Expression der Adhäsionsmoleküle und dadurch eine Forderung der Leukozytenadhäsivität mit konsekutiven Gewebe-Mikroverletzungen (Butler et al. 1993; Kalfin et al. 1993).

\subsubsection{Medikamentöse therapeutische Intervention}

Aufgrund der in den vorangehenden Kapiteln ausführlich beschriebenen Pathophysiologie und der systemischen Entzündungsreaktion als Folge einer EKZ ergeben sich auf unterschiedlichen Ebenen Ansätze für eine präoperative Applikation von Glucocorticoiden (Boldt et al. 1986; Westaby 1987; Butler et al. 1993; Miranda et al. 1982):

1. Verbesserung der Makro- bzw. Mikrozirkulation durch prä- und postkapilläre Vasodilatation mit Verhinderung kapillärer Stase und Thrombozytenaggregation (Piepenbrock et al. 1977; Sellevold und Jynge 1985). Hierbei spielt sowohl eine direkte Gefäßwirkung als auch die Hemmung der Phospholipase A eine Rolle, wodurch die Bildung hochaktiver Reaktionsprodukte (Arachidonsäure) mit stark vasokonstriktorischer Potenz verhindert wird (Möllmann et al. 1984).

2. Begrenzung der Komplement-induzierten Granulozytenaggregation (Westaby 1987; Butler et al. 1993).

3. Direkte Abdichtung der Kapillarendothelien und damit Bekämpfung einer deletären Überflutung des Interstitiums mit Flüssigkeit (Sibbald et al. 1981; Wilson 1972).

4. Verhinderung der Freisetzung aktiver biogener Amine durch Stabilisierung der Leukozyten-, Thrombozyten- und lysosomalen Membranen (Wilson 1972).

5. Supprimierung der TNF- und $\mathrm{LTB}_{4}$-Freisetzung sowie der t-PA-Aktivität während der Reperfusionsphase des Herzen und der Lunge (Jansen et al. 1991a).

Die Glucocorticoid-Dosierung der vorliegenden Studie wurde aufgrund bisheriger Studien gewählt. In der Literatur reicht die Dexamethason-Gabe von $1 \mathrm{mg} \cdot \mathrm{kg}^{-1}$ (Jansen et al. 1991a; Jansen et al. 1991b; Miranda et al. 1982) bis zu $4 \mathrm{mg} \cdot \mathrm{kg}^{-1} \mathrm{Körpergewicht} \mathrm{(Sibbald} \mathrm{et} \mathrm{al.} \mathrm{1981).}$

\subsection{Intravaskuläre Flüssigkeitvolumina}

\subsection{1 „Gesamtes“ und ,zirkulierendes“ Blutvolumen}

Die Differenzierung zwischen „gesamtem“ und ,zirkulierendem“ Blutvolumen basiert auf unterschiedlichen Meßverfahren. Das $V_{\text {d-ges }}$ wird in vitro aus der ICG-Eliminationskinetik nach spektralphotometrischer Analyse der über $30 \mathrm{~min}$ nach Farbstoffinjektion entnommenen Blutproben berechnet (vgl. Abschnitt 3.4.4). Die Bestimmung von $V_{d-\text { zirk }}$ erfolgt über einen rekursiven Faltungsalgorithmus aus den aortalen Farbstoffdilutionskurven, die fiberoptisch über vier Minuten nach Bolusinjektion in vivo registriert werden ( $v g l$. Abschnitt 2.6).

Das Verfahren zur Messung des Blutvolumens auf der Basis einer Kreislauf-Transportfunktion und eines rekursiven Faltungsalgorithmus erfaßt nur einen Teil des gesamten Blutvolumens, das 
durch das in vitro Verfahren gemessen wird. Dieser Anteil entspricht vermutlich dem im Beobachtungszeitraum ,zirkulierenden“ Anteil des Blutvolumens, d.h. es handelt sich um den Teil des Blutvolumens, der effektiv für den Kreislauf zur Verfügung steht. Das „gesamte“, in vitro bestimmte Blutvolumen, umfaßt hingegen auch solche Blutvolumenanteile („Reservevolumen“), die nicht oder nur sehr verzögert an der Zirkulation teilnehmen. Das „zirkulierende“ Volumen stellt somit einen „dynamischen“ Anteil an dem Gesamtvolumen dar und wird sich in Abhängigkeit vom Herzzeitvolumen soweit den aktuellen hämodynamischen Bedürfnissen anpassen wie das „Reservevolumen“ dies zuläßt.

Bei physiologischen Kreilaufverhältnissen besteht offensichtlich ein relativ konstantes Verhältnis zwischen dem „zirkulierenden“ und dem „gesamten“ Blutvolumen. Jedoch treten unter pathophysiologischen Gesichtspunkten, wie zum Beispiel im Rahmen einer Herzoperation, aufgrund der operationsbedingten Volumenverschiebungen (Herz-Lungen-Maschine, Blut- und Flüssigkeitsverluste) instabilere Kreislaufsituationen auf, die das Verhältnis zwischen den beiden Größen beeinflußt. Insbesondere unter diesen instabilen Kreilaufverhältnissen, die in der Regel als Volumenmangel interpretiert werden und mit Volumensubstitution zu behandeln sind, ist nicht das totale Blutvolumen die entscheidende Größe, sondern das für die Zirkulation zur Verfügung stehende Volumen.

\subsubsection{Korrelation zwischen ,zirkulierendem" und,gesamtem" Blutvolumen}

Über alle Meßzeitpunkte zeigte sich eine deutlich lineare Beziehung zwischen dem „zirkulierenden“ und dem „gesamten“ Blutvolumen (s. Abbildung 6-1) mit einem Korrelationskoeffizient von 0,56 und 0,57 in der PLC- und der DXM-Gruppe entsprechend. Nach theoretischen Überlegungen ist aber eine lineare Beziehung nicht immer zu erwarten, denn das $V_{d-\text { zirk }}$ könnte sich bedarfsorientiert durch Mobilisation von Volumenanteilen aus dem „Reservevolumen“ den jeweiligen hämodynamischen Bedürfnissen anpassen, ohne daß sich das $V_{d \text {-ges }}$ ändert.

Betrachtet man nur die präoperativen Werte der beiden Patientengruppen, ergibt sich ein Korrelationskoeffizient von 0,69. Postoperativ scheint jedoch sowohl die Anpassung des Kreislaufs an die aktuellen hämodynamischen Verhältnisse als auch die präoperative Gabe von Dexamethason die Beziehung zwischen beiden Größen zu beeinflussen. Demzufolge betrug der Korrelationskoeffizient eine Stunde (1h) nach Beendigung der Operation in der Placebo-Gruppe 0,77 und in der Dexamethason-Gruppe 0,91 und zum Meßzeitpunkt $6 h$ 0,60 und 0,71 entsprechend. Zwanzig Stunden (20h) postoperativ fand sich nur noch eine schwache Korrelation zwischen dem $V_{\text {d-zirk }}$ und dem $V_{\text {d-ges. }}$. 

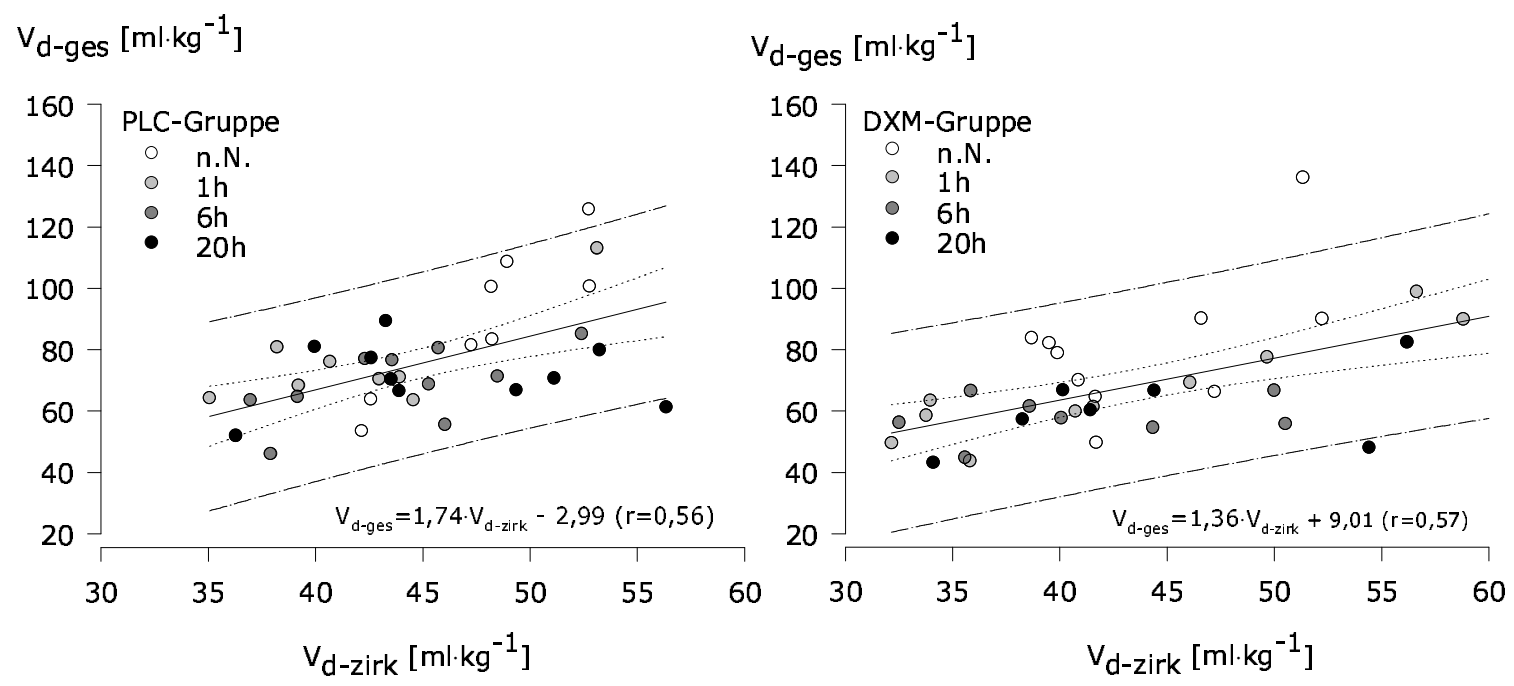

Abbildung 6-1. Korrelation von ,gesamtem“ und „zirkulierendem“ Blutvolumen

PLC-Gruppe $=$ Placebo-Gruppe $(n=10) ;$ DXM-Gruppe $=$ Dexamethason-Gruppe $(n=10)$

n.N. = nach Narkoseeinleitung; $1 \mathrm{~h}, 6 \mathrm{~h}, 20 \mathrm{~h}=$ eine, sechs, zwanzig Stunden post-OP

$V_{\text {d-ges }}=$,gesamtes“ Blutvolumen; $V_{d-\text { zirk }}=$,,zirkulierendes“ Blutvolumen

Ein guter Korrelationskoeffizient stellt allerdings kein Maß über die Güte der Methoden zur Erfassung von Blutvolumina dar, weil mit den beiden Verfahren definitionsgemäß nicht deckungsgleiche Verteilungsräume bestimmt werden. Vielmehr zeigt wahrscheinlich eine gute Korrelation in der frühen postoperativen Phase und vor allem in der Dexamethason-Gruppe unter der gegebenen Voraussetzungen einer linearen Beziehung, daß der Parameter $V_{d-\text { zirk }}$ während dieser Phasen besser Änderungen im ,gesamten“ Blutvolumen widerspiegelt.

\subsubsection{Absolute Größe und relative Änderungen der Verteilungsvolumina}

Im zeitlichen Verlauf zeigte das ,zirkulierende“ Blutvolumen $\left(V_{d-\text { zirk }}\right)$ in der DexamethasonGruppe keine wesentlichen Änderungen (s. Abbildung 6-2). Demgegenüber lagen die Werte der Patienten in der Placebo-Gruppe von der ersten bis zur sechsten postoperativen Stunde deutlich unter den Ausgangswerten mit maximaler Senkung um ca. $10 \%$ zum Meßzeitpunkt $1 \mathrm{~h}$ ( $\mathrm{vgl}$. Abbildung 6-3). Das „gesamte“ Blutvolumen ( $\left.V_{\text {d-ges }}\right)$ fiel jedoch in beiden Gruppen bereits eine Stunde nach Operationsende stark ab und verblieb im weiteren Untersuchungszeitraum auf diesem niedrigen Niveau, ohne den Ausgangsbereich wieder zu erreichen (s. Abbildung 6-2). Zwar wurde auch bei den Patienten der vorliegenden Studie ein Abfall des arteriellen Blutdrucks während der Narkoseeinleitung mit Sufentanil in der Regel mit Volumengabe begegnet ( $\mathrm{vgl}$. Abschnitt 8.2). Dieser deutlicher Abfall des „gesamten“ Blutvolumens könnte aber allein durch die präoperative Volumensubstitution und in Anbetracht der hochpositiven intraoperativen Flüssigkeitsbilanzen ( $\mathrm{gll}$. Tabelle 4-6) nicht begründet werden. 
Blutvolumen $\left[\mathrm{ml} \cdot \mathrm{kg}^{-1}\right.$ ]

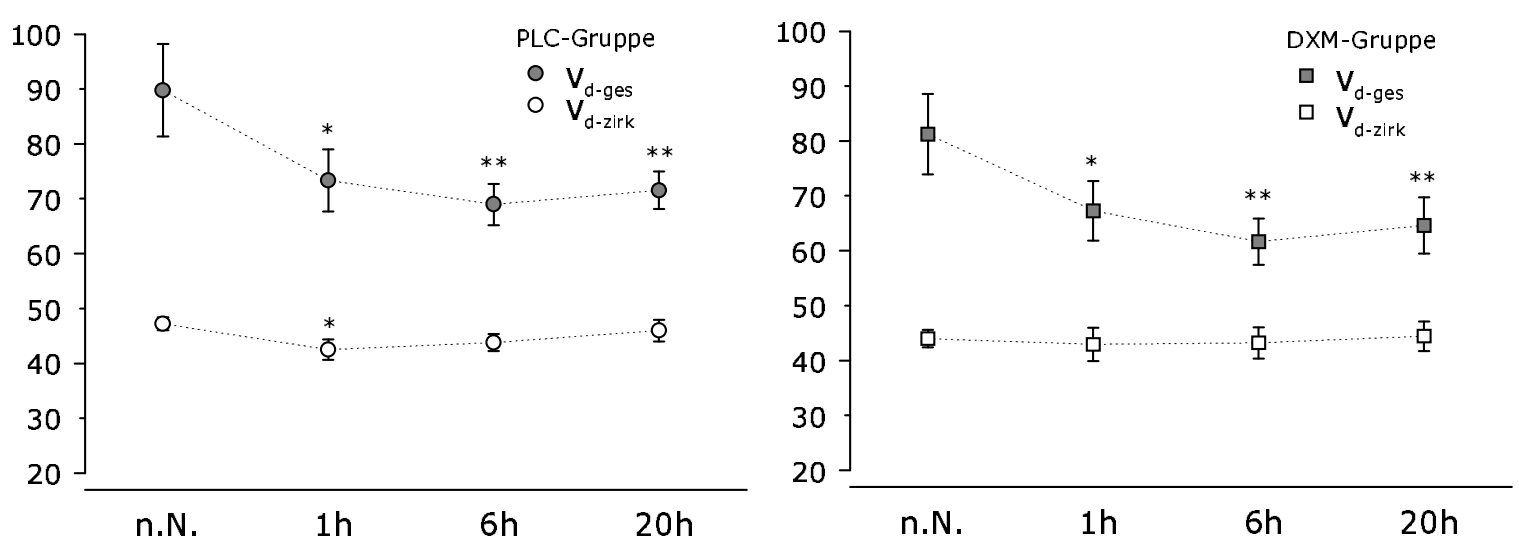

Abbildung 6-2. Vergleich der zeitlichen Verläufe vom „gesamten“ und „zirkulierenden“ Blutvolumen $(\bar{x} \pm \mathrm{sem})$

PLC-Gruppe $=$ Placebo-Gruppe $(n=10) ;$ DXM-Gruppe $=$ Dexamethason-Gruppe $(n=10)$

n.N. = nach Narkoseeinleitung; $1 \mathrm{~h}, 6 \mathrm{~h}, 20 \mathrm{~h}=$ eine, sechs, zwanzig Stunden post-OP

$V_{\text {d-ges }}=$,gesamtes“ Blutvolumen; $V_{d-\text { zirk }}=$,,zirkulierendes“ Blutvolumen

$*(* *) \quad: \mathrm{p}<0,05(0,01): 1 \mathrm{~h}, 6 \mathrm{~h}, 20 \mathrm{~h}$ vs. n.N.

Vielmehr spiegelt der Verlauf vom „gesamten“ Blutvolumen in beiden Gruppen eindrucksvoll einen latenten postoperativen intravasalen Volumenmangel trotz exzessiver NettoFlüssigkeitsbilanz (vgl. Abbildung 6-6) wider. Insbesondere zum Meßzeitpunkt $6 \mathrm{~h}$ ging die Ausweitung des Intravasalraumes bei beiden Gruppen mit einer Senkung des peripheren Gefäßwiderstandes einher, die den zusätzlichen Abfall des $V_{d \text {-ges }}$ während dieser Phase erklären könnte. Gleichzeitig stieg der Herzzeitvolumen-Index und in der Placebo-Gruppe noch zusätzlich die aortale Bluttemperatur von $35,01 \pm 0,32{ }^{\circ} \mathrm{C}$ zum Zeitpunkt $n . N$. auf $37,39 \pm 0,52{ }^{\circ} \mathrm{C}$ 6 Stunden postoperativ an (Tabelle 4-3, S. 38). Diese Konstellation repräsentiert eine hyperdyname Kreislaufsituation wie sie unter verschiedenen pathophysiologischen Bedingungen bekannt ist. Gerade im Rahmen einer Herzoperation unter dem Einsatz der extrakorporalen Zirkulation ist eine solche Kreislaufreaktion im wesentlichen als Ausdruck eines Postperfusionssyndroms, welches ausführlich im Kapitel 6.1 diskutiert wurde, anzusehen.

Wie in den oberen Abschnitten erläutert wurde, ist jedoch in der frühen Phase nach dem Eingriff aufgrund der instabilen Kreislaufverhältnisse nicht das $V_{\text {d-ges }}$ die entscheidende Größe, sondern das für die Zirkulation zur Verfügung stehende Volumen. Demzufolge ist es in dieser Phase von entscheidender Bedeutung, inwieweit und in welchem Ausmaß eine Inanspruchnahme des „Reservevolumens“, welches die Differenz zwischen $V_{\text {d-ges }}$ und $V_{d-\text {-zirk }}$ darstellt, zur Aufrechterhaltung des ,zirkulierenden“ Volumenanteils stattfindet. Der Anteil des $V_{d-z i r k}$ am $V_{\text {d-ges }}$ betrug bei beiden Gruppen unter Berücksichtigung zunächst nur der präoperativen Werte ca. $55 \%$. 

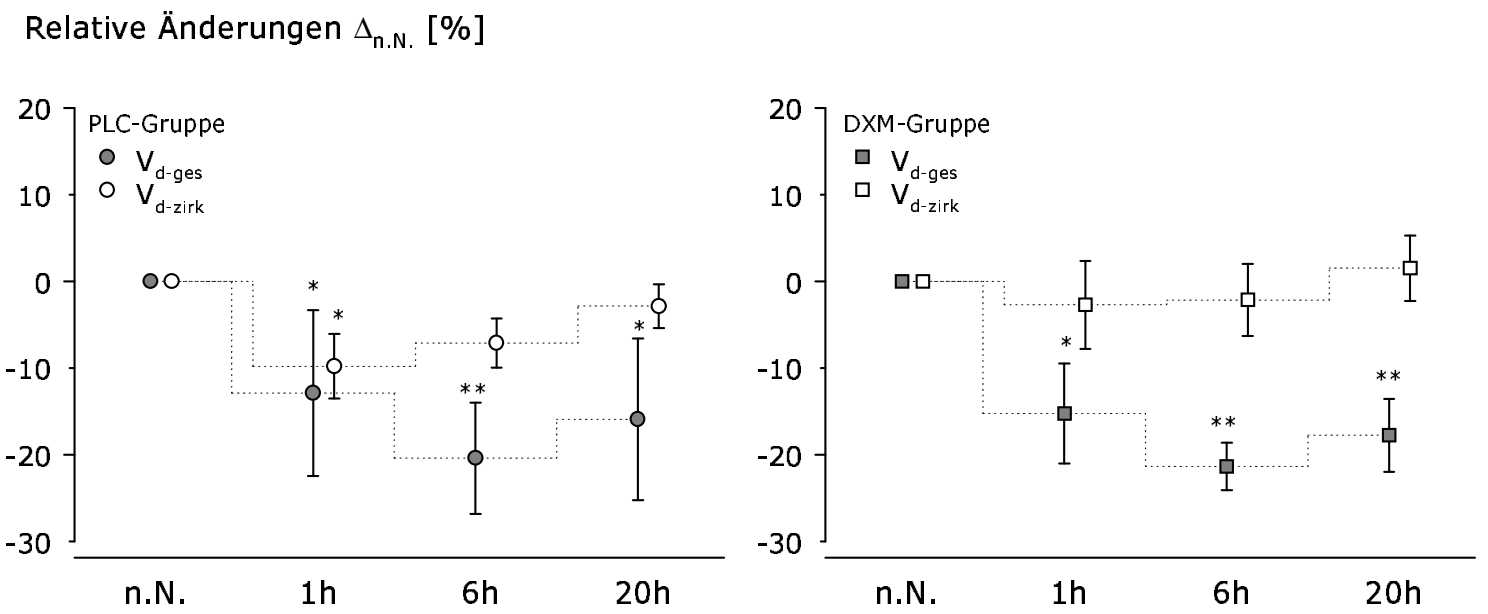

Abbildung 6-3. Relative prozentuale Änderungen vom „gesamten“ und „zirkulierenden“ Blutvolumen gegenüber dem Ausgangspunkt (n.N.), $\bar{x} \pm$ sem

PLC-Gruppe $=$ Placebo-Gruppe $(n=10) ;$ DXM-Gruppe $=$ Dexamethason-Gruppe $(n=10)$

n.N. = nach Narkoseeinleitung; $1 \mathrm{~h}, 6 \mathrm{~h}, 20 \mathrm{~h}=$ eine, sechs, zwanzig Stunden post-OP

$V_{\text {d-ges }}=$,,gesamtes“ Blutvolumen; $V_{d \text {-zirk }}=$,zirkulierendes“ Blutvolumen;

$\Delta_{n . N .}=$ Relative Änderungen der Volumina zum Ausgangswert (n.N.)

$*(* *) \quad: \mathrm{p}<0,05(0,01): 1 \mathrm{~h}, 6 \mathrm{~h}, 20 \mathrm{~h}$ vs. n.N.

Betrachtet man die relativen prozentualen Änderungen vom „gesamten“ und „zirkulierenden“ Blutvolumen gegenüber dem Ausgangspunkt (s. Abbildung 6-3), erwiesen die Patienten der Placebo-Gruppe zum Meßzeitpunkt $1 h$ trotz positiverer Netto-Flüssigkeitsbilanz verglichen zu den DXM-Patienten (Abbildung 6-6) sowohl eine Senkung des $V_{d \text {-ges }}$ als auch einen bedeutenden signifikanten Abfall des $V_{d-z i r k}$. Darüber hinaus betrug der Anteil des $V_{d-z i r k}$ am $V_{d-\text { ges }}$ zu diesem Zeitpunkt in der Placebo-Gruppe ca. 55\%. Diese Konstellation deutet auf eine tendenziell stärkere Inanspruchnahme des „Reserveblutvolumens“ hin. Außerdem stieg in dieser Phase bei konstantbleibendem Gefäßwiderstands-Index der Herzzeitvolumen-Index um ca. 30 \% gegenüber dem Ausgangswert und verblieb im weiteren Untersuchungszeitraum auf diesem erhöhten Niveau, wobei ein Mehrbedarf an medikamentöser Unterstützung der Inotropie während des gesamten postoperativen Verlaufs innerhalb der Placebo-Gruppe zu vermerken ist ( $s$. Tabelle 4-4, S. 39). Eindrucksvoll ist jedoch, daß 6 Stunden nach Beendigung der Operation trotz des abgefallenen Gefäßwiderstands-Index bei beiden Gruppen um ca. $25 \%$ ebenfalls in der PlaceboGruppe eine „Bedarfssteigerung“ von ungefähr $8 \%$ vom Anteil des ,zirkulierenden“ am „gesamten“ Blutvolumen stattgefunden hat. Demzufolge konnte auch in dieser Gruppe eine Steigerung des an der Zirkulation teilnehmenden Blutvolumens während der späten postoperativen Phase erreicht werden.

Im Gegensatz zur Placebo-Gruppe konnte zum Meßzeitpunkt $1 h$ bei den Dexamethason-Patienten eine ausreichende Mobilisation von Blutvolumen aus dem „Reservepool“ stattfinden und eine Aufrechterhaltung des ,zirkulierenden“ Volumens während der gesamten Untersuchungsphase gewährleistet werden. Der Anteil des $V_{d-z i r k}$ machte in dieser Gruppe eine Stunde nach der Operation ca. $65 \%$ des $V_{\text {d-ges }}$ aus. Die Mobilisierung von ,zirkulierendem“ Blutvolumen konnte 
zu einer Steigerung des Herzzeitvolumen-Index beitragen, die ca. $20 \%$ zum Meßzeitpunkt $1 \mathrm{~h}$ und $40 \%$ zum Zeitpunkt $6 h$ des Ausgangs- $H Z V$ betrug.

Zusammengefaßt ist also den erhobenen Daten zu entnehmen, daß sich die Placebo-Patienten in der frühen postoperativen Phase nahe an der Leistungsgrenze ihrer hämodynamischen Kompensationsmechanismen befanden. Auch eine größere Streuung der relativen Änderungen vom $V_{\text {d-ges }}$ innerhalb dieser Gruppe (Abbildung 6-3) repräsentiert ebenso eine instabilere Kreislaufsituation. Offensichtlich war die Auswirkung der protrahierten Volumenmangel aus den klassischen hämodynamischen Parametern zur Beurteilung des Volumenstatus und dem klinischen Bild nicht erkennbar, so daß die Patienten einen im allgemeinen unauffälligen postoperativen Verlauf aufwiesen. Andererseits scheint die Fähigkeit der mit Glucocorticoiden vorbehandelten Patienten eine ausreichende Volumenmenge aus der „Reserve“ mobilisieren zu können, den veränderten Bedürfnissen in der frühen postoperativen Phase in befriedigendem Maße begegnet zu sein.

\subsubsection{Blutvolumen im ,zentralen“" und ,peripheren“ Kreislauf}

Die Regulation des Blutvolumens erfolgt nach den heutigen Kenntnissen mit Hilfe spezieller Baro-Volumenrezeptoren, die sich ausschließlich in der Wand intrathorakaler Venen befinden (Gauer 1972) und das Kreislaufzentrum im Hirnstamm über den jeweiligen Zustand des Kreislaufsystems informieren. Diese Rezeptoren erfassen Änderungen der Wanddehnung der intrathorakal gelegenen Strukturen und dadurch indirekt Änderungen der Blutfülle des „zentralen“ Gefäßbettes. Über Aktivierung verschiedener Kreislaufmechanismen wird unter physiologischen Bedingungen eine Konstanthaltung dieser Blutfülle, d.h. des ,zentralen“ Blutvolumens angestrebt. Ferner fungiert das $V_{d-z e n t}$ als ein schnell mobilisierbares „Volumendepot“ für den linken Ventrikel. Bei akuten Steigerungen der Auswurfleistung des linken Ventrikels trägt das $V_{d-z e n t}$ dazu bei, ein mögliches Mißverhältnis zwischen der Förderleistung der Ventrikel auszugleichen, bis sich aufgrund von Steigerungen des venösen Rückstroms auch das Schlagvolumen des rechten Ventrikels an die höhere Leistung anpassen kann (Schmidt und Thews 1990, S. 553).

Jedoch ist unter pathophysiologischen Verhältnissen, wie zum Beispiel während der frühen postoperativen Phase nach einer Herzoperation, eine Gegensteuerung bei akuten Veränderungen des Blutvolumens von entscheidender Bedeutung. Diese wird von komplexen Anpassungsvorgängen erreicht, die das Verhältnis zwischen Gafäßkapazität und Blutvolumen betreffen. Stärkere Änderungen der Gefäßkapazität werden durch vasomotorische Reaktionen im Bereich der Kapazitätsgefäße ausgelöst, insbesondere des Niederdrucksystems, das eine etwa 200fach größere Compliance als das arterielle System besitzt (Arndt 1986). Währenddessen wird die Größe des Blutvolumens sowohl durch die kapilläre Filtrations-Reabsorptionsrate als auch durch die renale Flüssigkeitsausscheidung in Relation zur Flüssigkeitsaufnahme bestimmt (Schmidt und Thews 1990, S. 539). Die wichtigen Anpassungsmechanismen greifen also nicht nur am kleinen, sondern vornehmlich am großen Kreislauf. Insofern betreffen die erzielten Veränderungen zunächst das „zirkulierende“ Blutvolumen. Das $V_{d-z i r k}$ beinhaltet allerdings das „zentrale“ 
Blutvolumen, so daß das $V_{d-z e n t}$ auf Kosten des ,peripheren“ Blutvolumens $\left(V_{d-p e r}\right)$ reguliert wird, welches den dem großen Kreislauf zuzuordnenden Anteil von $V_{d-z i r k}$ darstellt.

Das „zentrale“ Blutvolumen wird von einigen Autoren als idealer Leitparameter für den intravaskulären Volumenstatus und zur Steuerung einer Volumentherapie angesehen (Pfeiffer et al. 1986; Pfeiffer 1990; Lichtwark-Aschoff et al. 1992). Pfeiffer (1990) beschreibt, daß bei den meisten pathologischen Zuständen das ,zentrale“ Blutvolumen durch die physiologischen Regulationsmechanismen so lange wie möglich konstant gehalten wird. Demzufolge gewährleistet eine ausreichende Auffüllung dieses Kompartimentes bei intakten körpereigenen Mechanismen zur Regulierung des $V_{d-z e n t}$ das für die jeweilige Kreislaufsituation erforderliche Blutvolumen $\left(V_{d-z i r k}\right)$. Jedoch kann dieses $V_{d-z i r k}$ insbesondere während der frühen postoperativen Phase nach kardiochirurgischen Eingriffen erheblich variieren, weil in dieser Phase Kreislaufinstabilitäten erfahrungsgemäß in erster Linie mit Volumenzufuhr begegnet werden und eine bedeutende Flüssigkeitsumverteilung zwischen den verschiedenen Kompartimenten stattfindet.

Somit präsentiert ein normales $V_{d-z e n t}$ nicht mehr als eine erfolgreiche Kompensation, im Sinne einer adäquaten Blutauffüllung des zentralen Kompartimentes aus Anteilen des ,zirkulierenden“ Blutvolumens, bei einem Volumenüber- oder -unterschuß unbekannten Ausmaßes. Insofern ist das ,zentrale“ Blutvolumen allein nicht in der Lage den aktuellen Volumenstatus und gegebenenfalls latente oder lavierte Volumen-Imbalancen anzuzeigen. Desgleichen erlaubt das aktuelle $V_{d-z i r k}$ allein auch keinen Rückschluß bezüglich der Angemessenheit eines „hämodynamisch relevanten“ Gesamtvolumens für die jeweilige Kreislaufsituation (Schorn 1995, S. 76). Die Kenntnis der Verteilung des $V_{d-z i r k}$ zwischen großem und kleinem Kreislauf oder mit anderen Worten die Erfassung des $V_{d \text {-zent }}$ und seines komplementären Anteils $V_{d-p e r}$ erbringt jedoch einen erheblichen Informationsgewinn zur Beurteilung der jeweiligen Volumenkonstellation und hierdurch Steuerung der Volumentherapie.

Im zeitlichen Verlauf ( $s$. Abbildung 6-4) wiesen die Dexamethason-Patienten bei den vorliegenden Untersuchungen ein signifikanten Abfall des $V_{\text {d-zent }}$ zwischen den Zeitpunkten n.N. und $l h$ von 9,89 $\pm 1,75 \mathrm{ml} \cdot \mathrm{kg}^{-1}$ auf $8,09 \pm 1,24 \mathrm{ml} \cdot \mathrm{kg}^{-1}$ auf, welcher als Ausdruck eines relativen, jedoch weitgehend kompensierten intravaskulären Volumenmangels $\mathrm{zu}$ werten sein dürfte. $\mathrm{Zu}$ diesem Zeitpunkt war der periphere Widerstands-Index bereits um ca. $15 \%$ abgefallen, eine Auffüllung des aufgeweiteten peripheren Gefäßbettes hatte allerdings noch nicht stattgefunden. Im weiteren Verlauf erreichte das $V_{d-z e n t}$ erneut das Ausgangsniveau. Die Tatsache, daß der Anteil des ,zentralen“ am ,zirkulierenden“ Blutvolumen sowie das ,periphere“ Blutvolumen über die gesamte Meßphase eine beachtliche Konstanz aufwies, könnte als Zeichen einer zu allen Meßzeitpunkten kompensierten Kreislauflage angesehen werden. 

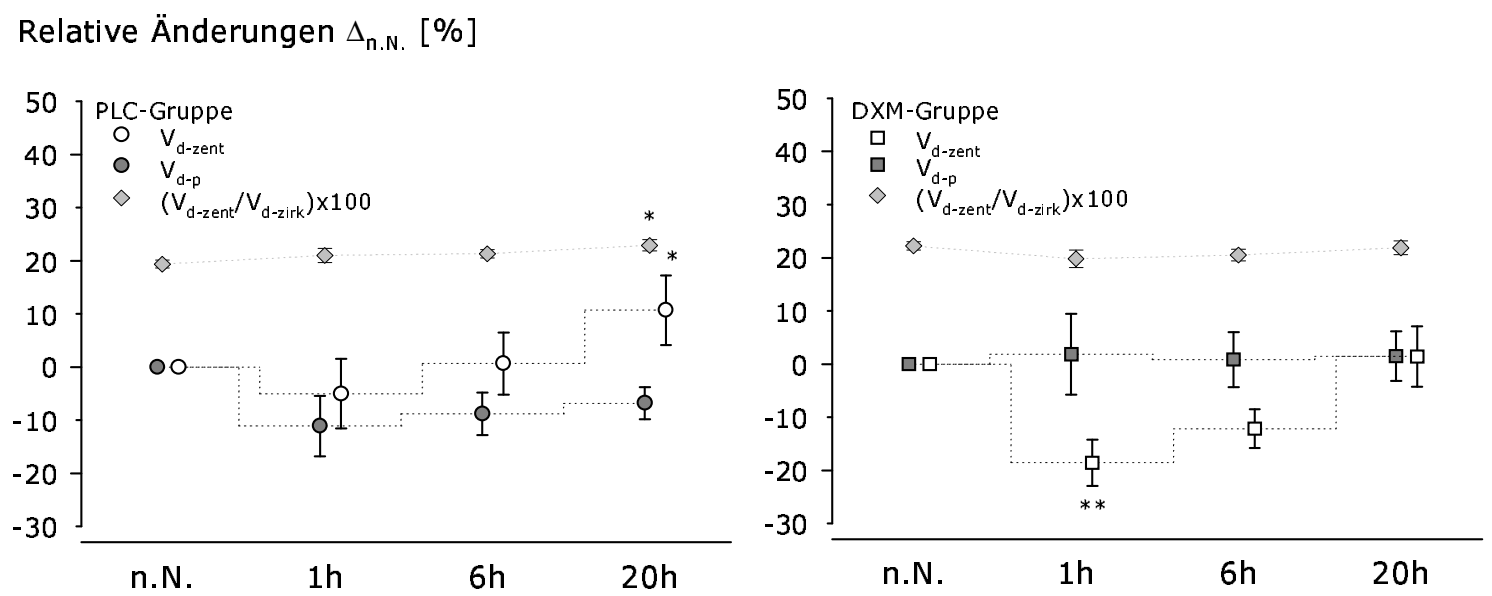

Abbildung 6-4. Relative prozentuale Änderungen von ,zentralem“ und „peripherem“ Blutvolumen gegenüber dem Ausgangspunkt (n.N.) sowie Anteil des ,zentralen“ am „zirkulierenden“ Blutvolumen $(\bar{x} \pm$ sem)

PLC-Gruppe $=$ Placebo-Gruppe $(\mathrm{n}=10) ;$ DXM-Gruppe $=$ Dexamethason-Gruppe $(\mathrm{n}=10)$

n.N. = nach Narkoseeinleitung; $1 \mathrm{~h}, 6 \mathrm{~h}, 20 \mathrm{~h}=$ eine, sechs, zwanzig Stunden post-OP

$V_{d-z e n t}=$,zentrales“ Blutvolumen; $V_{d-p}=$,,peripheres" Blutvolumen;

$V_{d-\text { zirk }}=$,,zirkulierendes“ Blutvolumen;

$\Delta_{n . N .}=$ Relative Änderungen der Volumina zum Ausgangswert (n.N.)

*(**) $\quad: \mathrm{p}<0,05(0,01): 1 \mathrm{~h}, 6 \mathrm{~h}, 20 \mathrm{~h}$ vs. n.N.

Andererseits zeigte das „zentrale“ Blutvolumen während der frühen postoperativen Phase in der Placebo-Gruppe keine größeren Veränderungen (s. Abbildung 6-4), jedoch stieg er bis zum Zeitpunkt $20 \mathrm{~h}$ signifikant um ca. $10 \%$ gegenüber dem Ausgangswert an. Der Anteil des „zentralen“ am „zirkulierenden“ Blutvolumen war zum diesem Zeitpunkt ebenso signifikant erhöht, welches als Hinweis einer Umverteilung des „zirkulierenden“ Blutvolumens in den kleinen Kreislauf anzusehen ist. Diese Beeinflussung der Verteilung des $V_{d-\text {-zirk }}$ zwischen großem und kleinem Kreislauf deutet allerdings nicht nur auf die Mobilisierung einer eventuell wieder verfügbaren „Volumenreserve“ hin. Sie könnte vielmehr als Folge einer während der späten postoperativen Phase hinzutretenden myokardialen Pumpschwäche zu bewerten sein.

Zudem lag das „periphere“ Blutvolumen bei den Placebo-Patienten zu allen Meßzeitpunkten unter dem Ausgangsniveau, da die aus dem „Reservevolumen“ mobilisierte Flüssigkeit vermutlich im Rahmen einer Zentralisation primär einer Bedarfssteigerung des „zentralen“ Kompartimentes diente. Dennoch geschah eine weitere Mobilisierung von Volumenanteilen aus dem „Pool“ zur Deckung eines vergleichsweise zur Dexamethason-Gruppe noch immer gesteigerten Durchblutungsbedarfs des ,peripheren“ Kompartimentes nicht, obwohl das Flüssigkeitsangebot aufgrund einer positiveren Gesamtbilanz in der Placebo-Gruppe größer war. Dies bestätigt nun gerade ohne weiteres, daß die myokardiale Pumpleistung zu diesem Zweck nicht ausreichte, trotzdem die medikamentöse Unterstützung der Inotropie in dieser Gruppe höher als die der Dexamethason-Patienten war.

Der zentraler Venendruck (ZVD) zeigte während der Messungen und zwischen den Gruppen keine statistisch relevanten Änderungen. Aus den erhobenen Daten zur Beurteilung des 
intravaskulären Volumenstatus ist dennoch erkennbar, daß das konventionelle, im wesentlichen auf der Registrierung von Druckwerten basierende hämodynamische Monitoring nicht die tatsächlichen Verhältnisse widerspiegelt.

\subsubsection{Flüssigkeitskompartimente des großen Kreislaufs}

In den einzelnen Organkreisläufen sind die theoretisch möglichen Durchblutungssteigerungen verschieden stark ausgeprägt. Bemerkenswert ist, daß in den Gefäßgebieten mit stark wechselnden funktionellen Anforderungen (Skelettmuskulatur, Gastrointestinaltrakt, Leber und Haut) die relativ größten Durchblutungsänderungen auftreten können. Demgegenüber wird die Durchblutung von lebenswichtigen Organen wie Herz, Nieren und Gehirn mit ständig hohen, aber relativ weniger stark wechselnden Anforderungen durch spezielle Autoregulationsmechanismen weitgehend konstant gehalten und innerhalb bestimmter Grenzen sogar von stärkeren Änderungen des Blutdrucks nur wenig beeinflußt (Schmidt und Thews 1990, S. 534). Durch ihre Fähigkeit, die Perfusion durch Anpassung des Durchflußwiderstandes über einen weiten Bereich des Perfusionsdruckes konstant zu halten, entziehen sie sich zum Beispiel bei einer „Zentralisation“ des Kreislaufs der allgemeinen Vasokonstriktion. Insofern wird unter pathophysiologischen Verhältnissen wie Volumenmangelzuständen ihre Durchblutung sogar auf Kosten der anderen Organsysteme aufrecht erhalten (Schmidt und Thews 1990, S. 533).

Mittels der Dispersionsanalyse des Farbstoffes ließen sich zu allen Meßzeitpunkten zwei vaskuläre Kompartimente charakterisieren, die sich signifikant bezüglich der mittleren Transitzeit von ICG und des relativen Gewichtes unterschieden (Tabelle 8-1, S. 82). Allerdings zeigten sich zwischen den Untersuchungsgruppen keine bedeutenden Unterschiede im Verhältnis der Kompartimente zueinander. Unter Ausgangsbedingungen ist aber den Berechnungen aus den Parametern der Transportfunktionen zu entnehmen, daß ein Kompartiment ca. $35 \%$ des „zirkulierenden“ Blutvolumens ausmachte, jedoch von durchschnittlich $60 \%$ des Herzzeitvolumens perfundiert wurde und daß ein zweites Kompartiment entsprechend komplementäre Werte für Ausdehnung und Durchblutung zeigte. Diese Konstanz der Volumen- und Flußrelationen unter Ausgangsbedingungen bestätigt jedoch, daß die errechneten Werte annähernd die tatsächlichen Verhältnisse widerspiegeln. Demnach repräsentiert das erste Kompartiment mit geringerer Ausdehnung und größeren Flußraten jene Organe, die eine hohe „Ruhedurchblutung“ aufweisen (Herz, Nieren und Gehirn). Das zweite Kompartiment wäre dann hauptsächlich den übrigen Kreislaufabschnitten inklusive des Splanchnikusgebietes zuzuordnen.

Bei allen berechneten Parametern, die die interkompartimentelle Volumen- und Flußverteilung beschreiben, konnten statistisch keine signifikante Unterschiede zwischen Placebo- und Dexamethason-Patienten gesichert werden.

In beiden Untersuchungsgruppen zeigten die berechneten Verteilungsräume sowohl für das „schnelle“ zentrale $\left(V_{d-1 k}\right)$ als auch für das „langsame“ periphere Kompartiment $\left(V_{d-2 k}\right)$ während der frühen postoperativen Phase keine wesentliche Änderungen (s. Abbildung 6-5 und Tabelle 4-5, S. 44). Ein leichter, jedoch nicht signifikanter Abfall dieser Volumina in der Placebo-Gruppe zum Meßpunkt $1 \mathrm{~h}$ konnte als Ausdruck des zu diesem Zeitpunkt abgefallenen „peripheren“ Blutvolumens zu werten sein (vgl. Abschnitt 6.2.2). Während der späten 
postoperativen Phase stieg allerdings in beiden Gruppen das Volumen des ersten Kompartimentes, das die wichtigen Organe zu versorgen vermag, signifikant an. 20 Stunden nach Beendigung der Operation lagen die Werte ca. $30 \%$ über dem Ausgangsbereich. Demgegenüber fiel das $V_{d-2 k}$ bei beiden Gruppen im Verlauf eher leicht ab und lag am ersten postoperativen Tag deutlich unter dem Ausgangsniveau.

Betrachtet man nun den relativen Verlauf der anteiligen Stromzeitvolumina in den Einzelkompartimenten, dann ist erkennbar, daß die bei beiden Untersuchungsgruppen erzielte Steigerung des Herzzeitvolumens während der frühen postoperativen Phase hauptsächlich der anteiligen Durchblutung des zweiten oder ,peripheren“ Kompartimentes diente ( $s$. Abbildung 6-5 und Tabelle 4-5, S. 44). Hingegen trug in der späteren Phase die Änderung von CI gegenüber dem Ausgangspunkt vorwiegend zur Änderung der Durchblutung des ersten, „zentralen“ Kompartimentes bei. Bemerkenswert ist jedoch, daß eine Steigerung des Stromzeitvolumens in der frühen postoperativen Phase zum „schnellen“ Kompartiment über den Mechanismus der Kreislauf-Zentralisation bei beiden Gruppen nicht erforderlich war, wie bei der Volumenmangel gemessen an dem Abfall des ,gesamten“ Blutvolumens zu erwarten wäre.

Aus den erhobenen Daten ist also zu folgern, daß

1. aufgrund der weitgehenden Stabilität der Änderungen vom Volumen und Stromzeitvolumen im schnellen Kompartiment über den Mechanismus der Autoregulation der ,zentralen“ Organe die Kreislauflage in der frühen postoperativen Phase bei beiden Gruppen als kompensiert anzusehen ist. Insbesondere den Patienten der Dexamethason-Gruppe gelang trotz des stärkeren Abfalls des $V_{\text {d-zent }}$ in dieser Phase ebenfalls eine ausreichende Mobilisation der Pool-Anteile zur Aufrechterhaltung der Perfusion des „schnellen“ Kompartimentes, das die Durchblutung der wichtigen ,zentralen“ Organe gewährleistet.

2. eine Stunde nach Operationsende das Splanchnikusgebiet der Placebo-Patienten vermutlich aufgrund eines reduzierten Volumenangebotes an das langsame Kompartiment (im Rahmen einer Verminderung des „peripheren“ Blutvolumens zu diesem Zeitpunkt) schlechter durchblutet wird. Trotz der ausreichenden Flüssigkeitszufuhr im Sinne einer hochpositiven Gesamtbilanz gelang den Patienten der Placebo-Gruppe eine Mobilisierung von Volumenanteilen aus dem „Reservepool“ zur ausreichenden Deckung des Flüssigkeitsbedarfs nicht.

3. am ersten Tag nach der Operation sowohl das mobilisierte Volumen als auch der gesteigerte Fluß dem schnellen Kompartiment zugute kam. Dies beweist einerseits, daß unter „stabileren“ Kreislaufverhältnissen eine eindeutige Beziehung zwischen lokaler Durchblutung und Durchflußwiderstand besteht. Andererseits aber auch, daß sich die Aufnahmekapazität der Einzelkompartimente für zugeführtes Volumen zeitlich ändert und sich an die jeweiligen Kreislauf-Bedürfnisse anpaßt. 

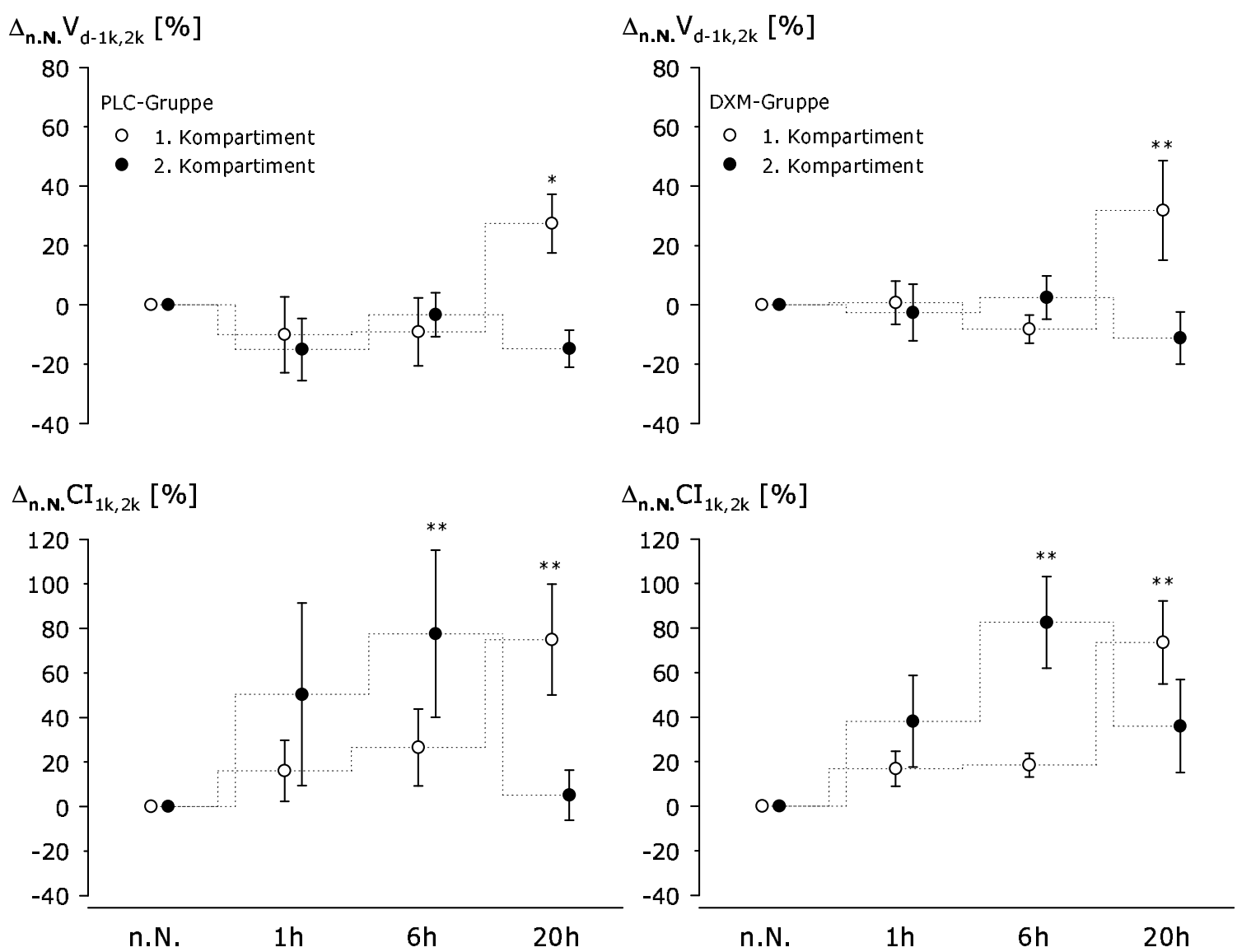

Abbildung 6-5. Relative prozentuale Änderungen von Volumen $\left(V_{d}\right)$ und Fluß $(C I)$ der Einzelkompartimente gegenüber dem Ausgangspunkt (n.N.), $\bar{x} \pm$ sem

PLC-Gruppe $=$ Placebo-Gruppe $(\mathrm{n}=10) ;$ DXM-Gruppe $=$ Dexamethason-Gruppe $(\mathrm{n}=10)$

n.N. = nach Narkoseeinleitung; $1 \mathrm{~h}, 6 \mathrm{~h}, 20 \mathrm{~h}=$ eine, sechs, zwanzig Stunden post-OP

$V_{d-l k, 2 k}=$ Volumen der Einzelkompartimente im Gesamtkreislauf;

$C I_{l k, 2 k}=$ Fluß zu den Einzelkompartimenten;

$\Delta_{n . N .}=$ Relative Änderungen von Volumen und Fluß zum Ausgangswert (n.N.)

$*(* *): \mathrm{p}<0,05(0,01)$ : 1h, 6h, 20h vs. n.N.

\subsection{Flüssigkeitsbilanzen und Volumenaustritt in den extravaskulären Raum}

In der terminalen Strombahn spielen beim Austausch von Flüssigkeit und Substanzen zwischen Blut und interstitiellem Raum Diffusionsvorgänge sowie Filtrations- und Reabsorptionsvorgänge in den beiden Richtungen eine wichtige Rolle. Dabei ist die Zahl der auswärts und einwärts diffundierenden Moleküle weitgehend gleich, so daß das Plasmavolumen der Kapillare praktisch konstant bleibt. Unter normalen Bedingungen liegt nach der klassischen Theorie von Starling (Schmidt und Thews 1990, S. 530) weitgehend ein Fließgleichgewicht zwischen den Flüssigkeitsmengen vor, die in den arteriellen Abschnitten der Kapillare filtriert und in den venösen Abschnitten reabsorbiert sowie über die Lymphgefäße abtransportiert werden. Filtration und Reabsorption in den Kapillaren hängt im wesentlichen vom einem transkapillären NettoDruckgradienten ab, der aus dem hydrostatischen Druck in den Kapillaren und in der 
interstitiellen Flüssigkeit sowie dem kolloidosmotischen Druck im Plasma und im Interstitium aufgebaut wird. Voraussetzung für den Aufbau der einzelnen Druckgradienten ist eine intakte semipermeable Grenzmembran zwischen Kapillarinnerem und Interstitium, die eine eingeschränkte Permeabilität für Moleküle ab einer bestimmten Größe (hauptsächlich ist das stark wasserbindende Albumin als Hauptteil der Eiweißfraktion betroffen) erlaubt.

Bei Störungen dieses Gleichgewichtes wie zum Beispiel im Rahmen einer Herzoperation ( $\mathrm{vgl}$. Abschnitt 6.1) treten Volumenverschiebungen zwischen intravaskulärem und interstitiellem Raum auf, die unter anderem im Hinblick auf die Bedeutung eines ausreichenden intravasalen Flüssigkeitsvolumens für die Kreislauffunktion wichtig sind. Durch Schädigung der besonders empfindlichen Kapillarmembran aufgrund der aktivierten inflammatorischen Mediatorsysteme entsteht eine Steigerung der Eiweißpermeabilität mit konsekutiver Senkung des kolloidosmotischen Druckgradienten, der in Verbindung mit einer postischämischen Erhöhung der intravaskulären hydrostatischen Druckwerte zu einer Flüssigkeitsbewegung in das Interstitium führt. Unter diesen Bedingungen wird es von der Kompensationfähigkeit der lymphatischen Drainagekapazität abhängen, ob und in welchem Ausmaß es zur Ausbildung eines interstitiellen Ödems kommt (Staub 1974).

Unter Berücksichtigung sowohl der applizierten kristalloiden und kolloiden Lösungen als auch der Zufuhr bzw. des Verlustes von Blutkomponenten, wurde für jede Untersuchungsgruppe die Netto-Gesamtflüssigkeitsbilanz berechnet ( $\mathrm{ggl}$. Tabelle 4-6) und in Abbildung 6-6 graphisch dargestellt. Bei allen Untersuchungspatienten zeigte sich eine bedeutende Flüssigkeitsretention, die sich zwar hauptsächlich während der Operation aber auch in der frühen postoperativen Phase ereignete und in der Placebo-Gruppe signifikant höher war. Das Fehlen von Dexamethason führte während des gesamten postoperativen Verlaufes zu einem höheren Volumenbedarf (besonders in Form von kristalloiden Lösungen) mit maximaler Flüssigkeitsakkumulation sechs Stunden (6h) nach Operationsende.

Mit einer signifikant besseren Diurese der Placebo-Patienten (vgl. Tabelle 4-6) konnten zwanzig Stunden (20h) postoperativ von diesem Überschuß an zugeführter Flüssigkeit $16 \%$ in der Placebo-Gruppe im Vergleich zu $11 \%$ in der Dexamethason-Gruppe ausgeschwemmt werden. Trotzdem lag bei den Placebo-Patienten die Netto-Bilanz am Ende des Meßzeitraums noch deutlich und signifikant höher im Vergleich zu der mit Glucocorticoiden vorbehandelten Gruppe.

Eine Expansion des Plasmavolumens durch die retinierten Flüssigkeitsmengen konnte bei beiden Untersuchungsgruppen nicht bewiesen werden. Das ,gesamte“ Plasmavolumen, das im Rahmen der Bestimmung des $V_{\text {d-ges }}$ berechnet wurde und seinem Verlauf widerspiegelt, blieb - nach einem primären Abfall eine Stunde ( $1 \mathrm{~h}$ ) postoperativ - stabil (vgl. Abbildung 6-2) und folgte nicht dem Verlauf der Flüssigkeitsbilanzierung. Vielmehr läßt sich vermuten, daß der Volumenüberschuß zum Teil die postoperativ aufgrund der leicht aber nicht signifikant abgefallenen peripheren Widerstände (vgl. Tabelle 4-3) aufgetretene relative Hypovolämie deckte, zum anderen aber zu einer Vergrößerung des extravaskulären Raums in Form eines interstitiellen Ödems führte. 


\section{Kumulative Gesamtbilanzen $\left[\mathrm{ml} \cdot \mathrm{kg}^{-1}\right]$}

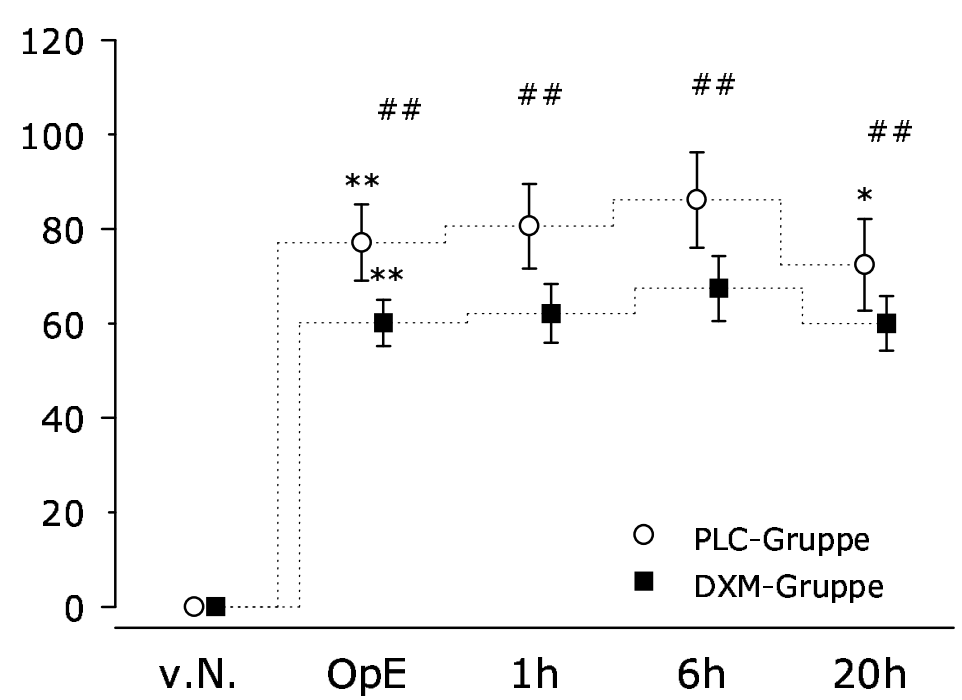

Abbildung 6-6. Kumulative Flüssigkeitsbilanzen der Patientengruppen ( $\bar{x} \pm$ sem)

PLC-Gruppe $=$ Placebo-Gruppe $(n=10) ;$ DXM-Gruppe $=$ Dexamethason-Gruppe $(n=10)$

v.N. = vor Narkoseeinleitung; OpE = Operationsende;

1h, $6 \mathrm{~h}, 20 \mathrm{~h}=$ eine, sechs, zwanzig Stunden post-OP

$*(* *) \quad: \mathrm{p}<0,05(0,01)$ : OpE vs. v.N., 1h vs. OpE, 6h vs. 1h, 20h vs. 6h

\#\# $\quad: \mathrm{p}<0,01:$ PLC $v s$. DXM

Eine Ein- und Ausfuhrbilanz für den gesamten extravaskulären Raum konnte als Differenz zwischen den Änderungen im „gesamten“ Plasmavolumen und den korrespondierenden NettoFlüssigkeitsbilanzen berechnet werden (Giannaris et. al. 1997; Schorn 1995, S. 92). Diese indirekte Größe, die dem Betrag der Flüssikeitsbewegung zwischen Intra- und Extravaskulärraum entspricht, wurde als „extravaskuläres Gesamtwasser“ $(E V G W)$ bezeichnet und sein Verlauf in der Abbildung 6-7 dargestellt.

Bei allen Untersuchungspatienten bestand zum Meßzeitpunkt $1 \mathrm{~h}$ ein bedeutender Flüssigkeitsaustritt in den extravaskulären Raum, der bis zur sechsten Stunde (6h) weiterhin zunahm. Diese Zunahme und insgesamt die Entstehung eines Ganzkörperödems während der frühen Phase nach Beendigung der Operation war allerdings in der Dexamethason-Gruppe deutlich schwächer ausgeprägt, wo sich bereits eine $(1 h)$ und sechs $(6 h)$ Stunden postoperativ zwischen den beiden Gruppen signifikante Unterschiede bei den Änderungen im „extravaskulären Gesamtwasser“ $(\triangle E V G W)$ zeigten. Die im Interstitium akkumulierte Flüssigkeit wurde erst in der späten postoperativen Phase und nur teilweise abgebaut. Sie betrug zum Meßzeitpunkt $20 \mathrm{~h}$ noch immer $55,56 \mathrm{ml} \cdot \mathrm{kg}^{-1}$ und 44,25 $\mathrm{ml} \cdot \mathrm{kg}^{-1}$ in der Placebo- und Dexamethason-Gruppe entsprechend, wobei sich die Gruppen zum Zeitpunkt $20 \mathrm{~h}$ statistisch nicht mehr signifikant unterschieden. 
"Extravaskuläres Gesamtwasser" $\left[\mathrm{ml} \cdot \mathrm{kg}^{-1}\right]$

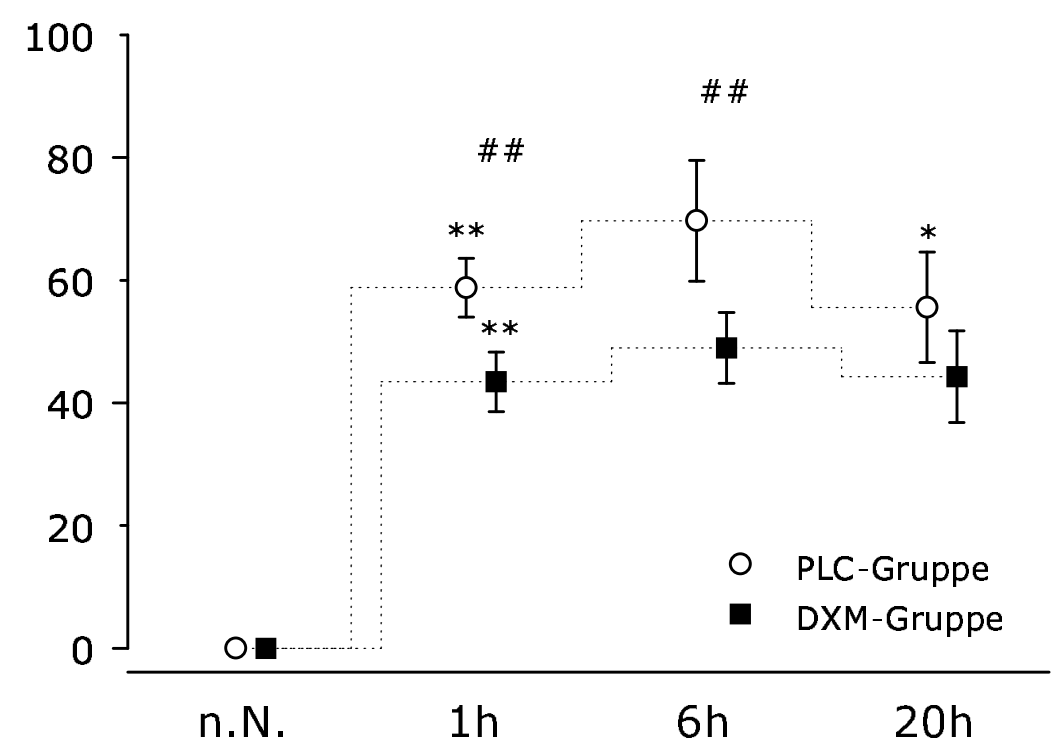

Abbildung 6-7. Absolute Änderungen des „extravaskulären Gesamtwassers“ ( $E V G W)$ gegenüber dem Ausgangspunkt (n.N.), $\bar{x} \pm$ sem

PLC-Gruppe $=$ Placebo-Gruppe $(\mathrm{n}=10) ;$ DXM-Gruppe $=$ Dexamethason-Gruppe $(\mathrm{n}=10)$

n.N. = nach Narkoseeinleitung; $1 \mathrm{~h}, 6 \mathrm{~h}, 20 \mathrm{~h}=$ eine, sechs, zwanzig Stunden post-OP

$*(* *) \quad: \mathrm{p}<0,05(0,01): 1 \mathrm{~h} v$ s. n.N., 6h vs. 1h, 20h vs. 6h

\#\# $:$ p $<0,01:$ PLC vs. DXM

Auch nach Wiederherstellung normaler intravaskulärer Druckverhältnisse kann ein solches interstitielles Ödem aufgrund bereits erfolgter Veränderungen der interstitiellen Membranen weiterhin bestehen (Comper 1984). Dadurch wird das Verhältnis zwischen Sauerstoffangebot und bedarf negativ beeinflußt und infolge einer konsekutiven Störung der Gewebsoxygenierung die Organfunktionen beeinträchtigt. Dexamethason wirkt der Entstehung und Ausprägung des Ödems entgegen, insofern ist seine präoperative Gabe von entscheidender Bedeutung.

Die Lunge ist unter dem Einfluß des kardiopulmonalen Bypasses als außerordentlich vulnerables Organ anzusehen und kann demzufolge unter dem Einsatz der Herz-Lungen-Maschine postoperativ eine Beeinträchtigung des pulmonalen Gasaustausches durch Einlagerung von Flüssigkeit in das interstitielle Bindegewebe der Lunge oder sogar Austritt von Flüssigkeit in die Alveolen beobachtet werden (Chenoweth et al. 1981; Kirklin et al. 1983; Boldt et al. 1986; Westaby 1987; Jansen et al. 1991a; Jansen et al. 1991b; Butler et al. 1993). Trotzdem zeigte sich erstaunlicherweise in beiden Patientengruppen anhand des zeitlichen Verlaufes des „extravaskulären Lungenwassers" ( $E V L W)$ keine bedeutende Akkumulation von Flüssigkeit im Lungeninterstitium, wie es entsprechend dem Verlauf des $E V G W$ zu erwarten wäre. Dies könnte als Folge eines durch die postoperative Überdruckbeatmung verminderten transmuralen hydrostatischen Druckgradienten zwischen Lungenkapillaren und Interstitium sein und wird von Studien, die eine postoperativ inhomogene Verteilung des interstitiellen Ödems im Organismus beschreiben, bestätigt (Schüpbach et al. 1978). Die Werte der Patienten in der Dexamethason-Gruppe lagen in der späten postoperativen Phase sogar signifikant niedriger gegenüber dem Ausgangspunkt und während des 
gesamten Meßzeitraums deutlich unter den Werten der Placebo-Patienten. Eine statistische Signifikanz bestand jedoch nur zum Meßzeitpunkt 20h (s. Abbildung 4-2 und Abbildung 6-8).

\section{"Extravaskuläres Lungenwasser" $\left[\mathrm{ml} \cdot \mathrm{kg}^{-1}\right]$}

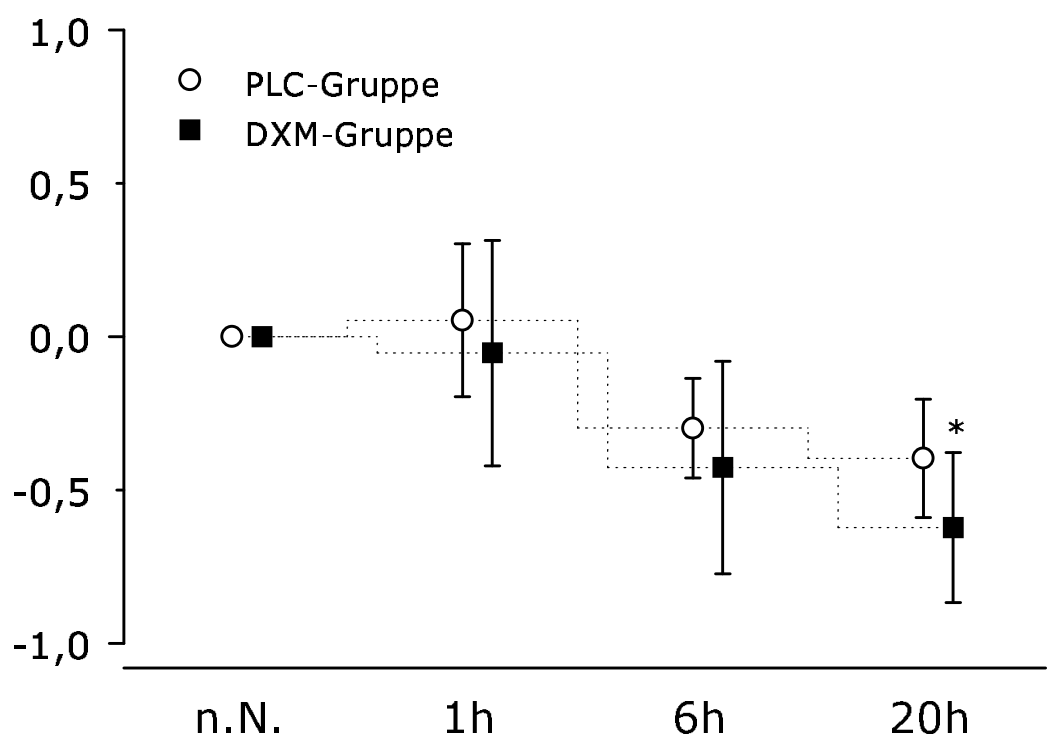

Abbildung 6-8. Absolute Änderungen des „extravaskulären Lungenwassers“ (EVLW) gegenüber dem Ausgangspunkt (n.N.), $\bar{x} \pm$ sem

PLC-Gruppe $=$ Placebo-Gruppe $(\mathrm{n}=10) ;$ DXM-Gruppe $=$ Dexamethason-Gruppe $(\mathrm{n}=10)$

n.N. = nach Narkoseeinleitung; $1 \mathrm{~h}, 6 \mathrm{~h}, 20 \mathrm{~h}=$ eine, sechs, zwanzig Stunden post-OP

$*: \mathrm{p}<0,05: 1 \mathrm{~h}, 6 \mathrm{~h}, 20 \mathrm{~h} v s . \mathrm{n} . \mathrm{N}$.

Der Verlauf der Drücke und der Gefäßwiderstände im kleinen Kreislauf scheint jedoch von der präoperativen Dexamethason-Applikation bedeutend beeinflußt zu sein. Patienten der PlaceboGruppe wiesen zu allen Meßzeitpunkten im Mittel höhere pulmonalarterielle Drücke und pulmonale Gefäßwiderstände verglichen zu den Dexamethason-Patienten auf, insbesondere während der frühen postoperativen Phase ( $\mathrm{vgl}$. Tabelle 4-3, S. 38). Der pulmonalkapilläre Verschlußdruck $(P C W P)$ stieg in der Placebo-Gruppe ebenfalls bis zum ersten postoperativen Tag an, mit Werten noch deutlich über dem Ausgangsniveau und signifikant höher gemessen an der Dexamethason-Gruppe. Durch derartige Veränderungen der pulmonalen Hämodynamik bei den Placebo-Patienten könnten die infolge einer unphysiologischen Perfusion während der $E K Z$ eingeleiteten Zirkulations- und Verteilungsstörungen im Bereich der terminalen Lungenstrombahn noch weiter verstärkt werden. Somit resultiert aufgrund der außerordentlichen Vulnerabilität der pulmonalen Strombahn ein frühzeitiger Perfusionsdefizit mit konsekutiver hypoxischer Schädigung des Kapillarendothels und eine generalisierte Kapillarwandinsuffizienz der Lunge. Aufgrund dessen kommt Maßnahmen, die die hämodynamischen Verhältnisse im kleinen Kreislauf positiv beeinflussen, eine wesentliche Bedeutung zu; zum Beispiel der präoperativen Glucocorticoid-Gabe. 


\subsection{Klinische Bedeutung der Ergebnisse}

Bei kritischer Beurteilung der in der vorliegenden Arbeit erhobenen Ergebnisse stellt sich zwangsläufig die Frage nach deren klinischer Wertigkeit.

Im Rahmen der intensivmedizinischen Betreuung chirurgischer Patienten kommt der perioperativen Flüssigkeitstherapie eine entscheidende Bedeutung zu. Ziel der therapeutischen Maßnahmen ist es, trotz teilweiser großer Flüssigkeitsverluste und -verschiebungen, einen adäquaten Füllungszustand des Kreislaufsystems zu gewährleisten. Die therapeutische Entscheidungsfindung unter Verwendung des konventionellen intensivmedizinischen Monitorings zur Steuerung der Volumentherapie stützt sich im wesentlichen auf indirekte hämodynamische Parameter wie arterieller Blutdruck, Herzfrequenz, zentraler Venendruck oder pulmonalkapillärer Verschlußdruck. Diese Parameter resultieren allerdings schon unter physiologischen Bedingungen aus dem Zusammenwirken verschiedener Regulationsmechanismen des Kreislaufs (Jacobson 1968; Shoemaker et al. 1988) und sind nicht in der Lage, den intravaskulären und erst recht nicht den extravaskulären Volumenstatus widerzuspiegeln.

Insbesondere unter pathophysiologischen Bedingungen, wie zum Beispiel während der frühen Phase nach einer Operation mit Einsatz der Herz-Lungen-Maschine, sind die physiologischen Regulationsmechanismen zur Aufrechterhaltung der intravasalen Volumenhomöostase durch pharmakologische Einflüsse im Rahmen der Narkose und durch die mediator-vermittelte „allgemeine unspezifische Entzündungsreaktion“ infolge des Blutkontaktes mit Fremdoberflächen beeinträchtigt (Moos und Craigo 1994; Schwinn et al. 1994; Chenoweth et al. 1981; Kirklin et al. 1983; Boldt et al. 1986; Westaby 1987; Haeffner-Cavaillon et al. 1989; Jansen et al. 1991a; Jansen et al. 1991b; Schorn et al. 1992; Butler et al. 1993; Elliott und Finn 1993; Schorn 1995).

Aufgrund dieser Störeinflüsse könnte ein erweitertes Monitoring des Volumenstatus mit Hilfe der angewandten Indikator-Verdünnungstechniken, insbesondere des bettseitig verfügbaren „zirkulierenden“ Blutvolumens, zum besseren Verständnis der Pathophysiologie und damit zur Entwicklung fundierter Interventionsmöglichkeiten und Behandlungsverfahren bei intensivmedizinischen Patienten beitragen. Tatsächlich gewinnen die gelieferten Informationen zusätzlich an Wichtigkeit bei oft älteren und multimorbiden Patienten beziehungsweise bei komplizierten Verläufen, wobei die körpereigenen Kompensationsmechanismen aufgrund einer vorbestehenden oder postischämisch hinzugetretenen myokardialen Insuffizienz häufig deutlich eingeschränkt sind.

Den Ergebnissen der vorliegenden Untersuchungen ist $\mathrm{zu}$ entnehmen, daß es bei beiden Untersuchungsgruppen während der frühen postoperativen Phase eine bedeutende Flüssigkeitsverschiebung in den extravaskulären Raum stattfindet. Bestätigen läßt sich dies zum einen durch die bis zur sechsten Stunde (Meßzeitpunkt 6h) nach Beendigung der Operation signifikant erhöhten Werte für das „extravaskuläre Gesamtwasser“. Zum anderen kommt es postoperativ trotz der hochpositiven Netto-Flüssigkeitsbilanzen zu einer nicht ausreichenden Auffüllung des Gefäßbettes mit konsekutiv relativen intravaskulären Volumenmangel. Offensichtlich folgt der Mediatorenaktivierung eine Störung der Integrität des Kapillarendothels, die zur unkontrollierten Extravasation von Flüssigkeit aus dem intravaskulären in den extravaskulären Raum führt 
(Blomqvist et al. 1990; Frostell et al. 1990; Wiener-Kronish und Broaddus 1993). Auf diese Weise entsteht ein generalisiertes interstitielles Ödem, das sowohl indirekt durch Beeinträchtigung der nutritiven Versorgung als auch direkt durch spezifische Funktionsbeeinträchtigung schädigend wirkt. Ein solches Ödem kann auch nach Reetablierung normaler Verhältnisse aufgrund einer bereits erfolgten Veränderung der interstitiellen Matrix persistieren (Comper 1984) und führt durch eine negative Beeinflussung des Verhältnisses zwischen Sauerstoffzufuhr und -verbrauch zu einer Störung der Gewebsoxygenierung und konsekutiven Organfunktionsstörungen (Chenoweth et al. 1981; Kirklin et al. 1983; Westaby 1987; Butler et al. 1993).

Deshalb kommt der präoperativen Applikation von Glucocorticoiden, die der Entstehung und Ausprägung des interstitiellen Ödems entgegenwirken (s. Kapitel 6.1.2, S. 63), eine wesentliche Bedeutung zu. Es konnte gezeigt werden, daß die Dexamethason-Vorbehandlung zu einer geringeren Flüssigkeitsextravasation während der frühen Phase nach Beendigung der Operation führt. In diesem Sinne wäre bei den Dexamethason-vorbehandelten Patienten zugleich eine klinisch bedeutend geringere Flüssigkeitsakkumulation im Lungeninterstitium zu erwarten. Jedoch zeigte der statistische Vergleich nicht nur zwischen beiden Gruppen sondern auch im zeitlichen Verlauf keine wesentlichen Unterschiede im extravaskulären Lungenwassergehalt $(E V L W)$. Möglicherweise wurde die Lungenwasserentstehung durch den postoperativ infolge der Überdrucksbeatmung (PEEP-Beatmung) verminderten transmuralen hydrostatischen Druckgradienten zwischen Lungenkapillaren und Interstitium positiv beeinflußt. Oder auch ist es Ausdruck einer inhomogenen organspezifischen Verteilung des interstitiellen Ödems im Organismus (vgl. Kapitel 6.3, S. 73). Dennoch erwiesen Placebo-Patienten zu allen Meßzeitpunkten und vorwiegend während der frühen postoperativen Phase im Mittel höhere Drücke und Gefäßwiderstände im kleinen Kreislauf. Durch derartigen Veränderungen der pulmonalen Hämodynamik könnten aber die infolge der „unphysiologischen“ HLM-Perfusion eingeleiteten Zirkulations- und Verteilungsstörungen im Bereich der terminalen Lungenstrombahn noch weiter verstärkt werden.

Eine komplette Unterdrückung der generalisierten Ödembildung gelingt aber allein durch Dexamethason nicht. Jedoch scheint eine präoperative Gabe für Patienten, für die wie in der vorliegenden Studie ein ,unkomplizierter Operationsverlauf“ zu erwarten ist (s. Einschlußkriterien unter Kapitel 3.1, S. 20), vertretbar zu sein. Darüber hinaus kommt es bei den relativ kurzen EKZ-Zeiten ( $s$. Tabelle 4-1, S. 35) zu keiner schwerwiegenden Beeinträchtigung der Kapillarschranke. Allerdings ist bei kardiochirurgischen Patienten mit vorbestehender beeinträchtigter Lungen- und Ventrikelfunktion eine Vorbehandlung mit Dexamethason empfehlenswert, um das Risiko einer vermehrten Wassereinlagerung in der Lunge und den anderen Organen mit folgenden Funktionsstörungen zu minimieren. Besonders bei älteren und multimorbiden Patienten oder bei komplizierten Verläufen mit deutlich längeren Bypass-Zeiten ist mit noch gravierenderen Veränderungen der Kapillarwandintegrität (Ratcliff et al. 1973) zu rechnen. Ob Dexamethason unter diesen Bedingungen eventuell in noch höheren Dosierungen oder zu einem anderen Applikationszeitpunkt einen noch günstigeren Effekt aufweist, müßte in zukünftigen Studien untersucht werden. 


\section{Zusammenfassung}

Die besondere Problematik der perioperativen Flüssigkeitstherapie bei kardiochirurgischen Patienten beruht auf den spezifischen Auswirkungen von Kardioplegie und extrakorporaler Zirkulation in Form von postischämischer myokardialer Funktionseinschränkung, Hämodilution und unspezifischer Entzündungsreaktion durch Mediatoraktivierung. Hierdurch wird einerseits die hämodynamische Anpassungsbreite eingeengt, andererseits werden alle Parameter modifiziert, die den transkapillären Flüssigkeits- und Eiweißtransport zwischen Intra- und Extravaskulärraum determinieren.

Mit den vorliegenden Untersuchungen sollte ein Beitrag zur klinischen Bedeutung einer präoperativen Gabe von Dexamethason erarbeitet werden. Ziel der Studie war zu untersuchen, inwieweit durch die präoperative Applikation von Glucocorticoiden die mediator-vermittelte Entzündungsreaktion supprimiert und dadurch die bedeutende Flüssigkeitsextravasation und Ödembildung günstig beeinflußt werden können.

Die Untersuchungen wurden an erwachsenen Patienten durchgeführt, die sich einer Myokardrevaskularisation unterzogen. Unter doppelblind-randomisierten Bedingungen erfolgte präoperativ die Zuteilung der Patienten in zwei Gruppen. Patienten der Placebo-Gruppe erhielten keine Glucocorticoide, die der Verum-Gruppe $1 \mathrm{mg} \cdot \mathrm{kg}^{-1}$ Dexamethason intravenös nach Einleitung der Narkose. Der extrakorporaler Kreislauf wurde mit einem zellfreien Volumen von 21 vorgefüllt (s. Kapitel 3.2.2, S. 22). Neben Erfassung hämodynamischer und Bestimmung laborchemischer Parameter erfolgten nach zentralvenöser Bolusinjektion des „gekühlten“ Farbstoffes Indocyaningrün (ICG) die Registrierungen von Indikatordilutions- bzw. -eliminationskinetiken für Kälte und ICG mit Hilfe eines pulmonalarteriellen Thermistor- und eines aortalen Fiberoptik-Thermistorkatheters. Die Messungen wurden jeweils unmittelbar nach Narkoseeinleitung sowie eine, sechs und 20 Stunden nach Ende der Operation durchgeführt.

Das „gesamte“ Blutvolumen $\left(V_{d-g e s}\right)$ berechnete sich unter Berücksichtigung des jeweiligen Hämatokrits aus der Eliminationskinetik des Farbstoffes, die durch ,in vitro“-Analyse der ICGPlasmakonzentrationen ermittelt wurde. Basierend auf der $I C G$-Verteilungskinetik und nach den Regeln der Stochastik wurde simultan das ,zirkulierende“ Blutvolumen $\left(V_{d-z i r k}\right)$ bestimmt, indem durch einen neu entwickelten rekursiven Faltungsalgorithmus ausgehend von der aortalen Farbstoffdilutionskurve eine Transportfunktion für den Gesamtkreislauf berechnet wurde. Zur Beschreibung der durch die Dispersionsanalyse ermittelten Transportfunktion war immer ein mehrkompartimentelles Modell erforderlich. Bei Zugrundelegen von zwei Kreislaufkompartimenten, wodurch einerseits die Durchblutung der ,zentralen“ Organe (Herz, Gehirn und Nieren) und andererseits die Perfusion des Splanchnikusgebietes repräsentiert wird, ließen sich in allen Fällen zufriedenstellende Anpassungsergebnisse der Modellsimulationen an die gemessenen Indikatorkinetiken erzielen. Das „zentrale“ Blutvolumen $\left(V_{\text {d-zent }}\right)$ und das „extravaskuläre Lungenwasser“ ( $E V L W)$ ergaben sich aus den mittleren Transitzeiten für $I C G$ und Kälte.

Aus Berechnungen der Flüssigkeitsbilanzen konnten zum extravaskulären Volumenstatus zusätzliche Informationen abgeleitet werden. Die Netto-Flüssigkeitsbilanzen ergaben sich aus der 
Ein- und Ausfuhr kristalloider und der auf eine $5 \%$ ige Protein-Standardlösung adjustierten Menge kolloider Lösungen. Die Differenz dieser Netto-Bilanz und der entsprechenden Änderungen des ,gesamten“ Plasmavolumens entsprach den Änderungen im ,extravaskulären Gesamtwasser" (EVGW).

Im Hinblick auf die klinische Fragestellung konnte gezeigt werden, daß durch die präoperative Applikation von Glucocorticoiden die erhebliche extravaskuläre Flüssigkeitsretention als Folge der herzchirurgischen Operation mit extrakorporaler Zirkulation signifikant reduziert wird. Insbesondere bei älteren und multimorbiden Patienten beziehungsweise bei komplizierten Verläufen könnte hierdurch einer Störung der Gewebsoxygenierung mit konsekutiver Organdysfunktion prophylaktisch entgegengewirkt werden.

Die Ergebnisse der durchgeführten Untersuchungen zeigen darüber hinaus, daß die erheblichen Veränderungen intravaskulärer und extravaskulärer Volumina durch ein routinemäßig hämodynamisches Monitoring nicht erfaßt werden. Ein erweitertes Monitoring des Volumenstatus mit Hilfe der angewandten Indikator-Verdünnungstechniken könnte allerdings zum besseren Verständnis der Pathophysiologie und damit zur Entwicklung fundierter Interventionsmöglichkeiten und Behandlungsverfahren bei intensivmedizinischen Patienten beitragen. 


\section{Anhang}

\subsection{Parameter der berechneten Kreislauf-Transportfunktionen}

\begin{tabular}{|c|c|c|c|c|c|}
\hline & & n.N. & $1 \mathrm{~h}$ & $6 \mathrm{~h}$ & $20 \mathrm{~h}$ \\
\hline \multirow{3}{*}{$\alpha$} & & \# & & & \\
\hline & PLC & $201,13 \pm 78,74$ & $179,11 \pm 80,72$ & $142,84 \pm 35,47 * *$ & $161,70 \pm 87,17$ \\
\hline & DXM & $235,66 \pm 127,20$ & $214,31 \pm 124,50$ & $181,95 \pm 118,34 * *$ & $157,91 \pm 104,89 * *$ \\
\hline \multirow[t]{2}{*}{$\sigma$} & PLC & $0,44 \pm 0,06$ & $0,38 \pm 0,04 * *$ & $0,38 \pm 0,05 * *$ & $0,39 \pm 0,07 *$ \\
\hline & DXM & $0,45 \pm 0,02$ & $0,41 \pm 0,05$ & $0,39 \pm 0,04 * *$ & $0,39 \pm 0,04 * *$ \\
\hline \multirow{2}{*}{$\begin{array}{c}\text { Mtt } \\
{[\mathrm{sec}]}\end{array}$} & PLC & $11,22 \pm 4,43$ & $8,34 \pm 1,14 * *$ & $8,11 \pm 1,34 * *$ & $8,94 \pm 3,41 * *$ \\
\hline & DXM & $10,83 \pm 1,41$ & $9,01 \pm 1,33^{*}$ & $8,22 \pm 1,24 * *$ & $8,45 \pm 1,16 * *$ \\
\hline \multirow[t]{2}{*}{$\alpha_{1}$} & PLC & $0,48 \pm 0,08$ & $0,51 \pm 0,19$ & $0,45 \pm 0,14$ & $0,58 \pm 0,08 *$ \\
\hline & DXM & $0,51 \pm 0,09$ & $0,50 \pm 0,09$ & $0,47 \pm 0,07$ & $0,59 \pm 0,10$ \\
\hline \multirow[t]{2}{*}{$\sigma_{1}$} & PLC & $0,29 \pm 0,02$ & $0,31 \pm 0,04$ & $0,29 \pm 0,05$ & $0,33 \pm 0,06 * *$ \\
\hline & DXM & $0,30 \pm 0,08$ & $0,32 \pm 0,07$ & $0,30 \pm 0,04$ & $0,36 \pm 0,06 * *$ \\
\hline \multirow{2}{*}{$\begin{array}{l}\mathrm{Mtt}_{1} \\
{[\mathrm{sec}]}\end{array}$} & PLC & $30,06 \pm 7,60$ & $22,17 \pm 6,20 * *$ & $21,19 \pm 5,07 * *$ & $23,23 \pm 5,36 * *$ \\
\hline & DXM & $29,14 \pm 5,87$ & $24,73 \pm 3,39 *$ & $22,42 \pm 3,03 * *$ & $23,00 \pm 3,97^{* *}$ \\
\hline \multirow[t]{2}{*}{$\alpha_{2}$} & PLC & $0,34 \pm 0,08$ & $0,35 \pm 0,20$ & $0,42 \pm 0,16$ & $0,27 \pm 0,07$ \\
\hline & DXM & $0,33 \pm 0,08$ & $0,35 \pm 0,10$ & $0,41 \pm 0,09$ & $0,31 \pm 0,09$ \\
\hline \multirow{2}{*}{$\begin{array}{c}\mathrm{mtt}_{2} \\
{[\mathrm{sec}]}\end{array}$} & PLC & $82,29 \pm 24,79$ & $61,65 \pm 24,15^{* *}$ & $51,80 \pm 15,30 * *$ & $66,20 \pm 16,61 *$ \\
\hline & DXM & $88,83 \pm 16,26$ & $67,49 \pm 15,52 * *$ & $55,06 \pm 10,62 * *$ & $65,54 \pm 14,10 * *$ \\
\hline \multirow{3}{*}{$\mathbf{R}$} & & & & & \#\# \\
\hline & PLC & $0,82 \pm 0,04$ & $0,86 \pm 0,04 *$ & $0,88 \pm 0,03 * *$ & $0,86 \pm 0,04 *$ \\
\hline & DXM & $0,83 \pm 0,04$ & $0,85 \pm 0,04$ & $0,89 \pm 0,05 * *$ & $0,90 \pm 0,03 * *$ \\
\hline \multirow{3}{*}{$\begin{array}{l}\text { MTT } \\
\text { [sec] }\end{array}$} & & & \# & & \\
\hline & PLC & $51,11 \pm 13,98$ & $35,14 \pm 7,03 * *$ & $34,59 \pm 6,61 * *$ & $36,61 \pm 9,39 * *$ \\
\hline & DXM & $51,57 \pm 8,32$ & $41,28 \pm 6,32 * *$ & $36,53 \pm 6,06 * *$ & $36,77 \pm 8,37 * *$ \\
\hline
\end{tabular}

Tabelle 8-1. Parameter der ermittelten Kreislauf-Transportfunktionen ( $\bar{x} \pm \mathrm{sd})$

PLC $=$ Placebo-Gruppe $(n=10) ;$ DXM = Dexamethason-Gruppe $(n=10)$

n.N. = nach Narkoseeinleitung; $1 \mathrm{~h}, 6 \mathrm{~h}, 20 \mathrm{~h}=$ eine, sechs, zwanzig Stunden post-OP

$\alpha, \sigma, m t t=$ Parameter der Primärkurve (,first pass“);

$\alpha_{1}, \sigma_{1}, m t_{l}=$ Parameter des schnellen Kompartimentes;

$\alpha_{2}$, tt $_{2}=$ Parameter des langsamen Kompartimentes;

$R=$ Rezirkulationsfraktion; $M T T=$ Kreislauftransitzeit;

$*(* *) \quad: \mathrm{p}<0,05(0,01): 1 \mathrm{~h}, 6 \mathrm{~h}, 20 \mathrm{~h} v s . \mathrm{n} . \mathrm{N}$.

\# (\#\#) : $\mathrm{p}<0,05(0,01):$ PLC vs. DXM 


\subsection{Narkoseeinleitung mittels Sufentanil}

Im Rahmen des gleichen Forschungsprojektes (Sonderforschungsbereich 330 der DFG) wurde zusätzlich zur vorliegenden Studie untersucht, ob der beobachtete Blutdruckabfall bei einer total intravenösen Einleitung der Narkose mit Sufentanil auf einen Abfall der intrathorakalen Füllungsvolumina und/oder auf eine Flüssigkeitsumverteilung zwischen den verschiedenen Körperkompartimenten zurückzuführen ist und inwieweit diese klinische Situation mit Volumensubstitution kompensiert werden kann.

Die Genehmigung zur Durchführung der Untersuchungen erfolgte durch die Ethik-Kommision der Universität Göttingen. Alle Patienten wurden umfassend über die Risiken aufgeklärt und erteilten anschließend schriftlich ihr Einverständnis. Es wurden 15 Patienten, die sich einer elektiven Myokardrevaskularisation unterzogen in die Studie aufgenommen. Bei allen Patienten wurde jeweils vor Einleitung der Narkose unter Lokalanästhesie ein kombinierter FiberoptikThermistor-Katheter in Seldinger Technik über die linke A. femoralis mit der Spitze in der Aorta descendens sowie ein Swan-Ganz-Einschwemmkatheter in der A. pulmonalis plaziert ( $\mathrm{vgl}$. Abschnitt 3.2.1).

Nach ausreichender Präoxygenierung wurde die Narkose mit $2 \mu \mathrm{g} \cdot \mathrm{kg}^{-1}$ Sufentanil und $0,1 \mathrm{mg} \cdot \mathrm{kg}^{-1}$ Körpergewicht Pancuroniumbromid eingeleitet. Es erfolgte die nasotracheale Intubation und eine kontrollierte normocarbische Beatmung bei einem $\mathrm{FiO}_{2}$ von 0,5. Angestrebt wurde ein $\mathrm{CO}_{2}$ zwischen 4,5 und $5 \mathrm{Vol} \%$ und eine pulsoximetrisch gemessene arterielle Sauerstoffsättigung von mehr als $95 \%$.

Zur Messung der Indikatorkinetiken wurden vor und nach Narkoseeinleitung jeweils zweimal $15 \mathrm{ml}$ eines eisgekühlten Farbstoffbolus (22,5 mg Indocyaningrün) durch das proximale Lumen des Pulmonalis-Katheters in den rechten Vorhof injiziert. Die Aufzeichnung und Auswertung der registrierten Indikatordilutionskurven erfolgte auf gleicher Basis zur im Kapitel 2 (S. 3) und 3 (S. 20) der vorliegenden Studie dargestellten Methodik.

Zusammengefaßt hatte die Einleitung der Narkose mit Sufentanil eine Umverteilung des Blutvolumens vom intrathorakalen zum peripheren Flüssigkeitskompartiment zur Folge. Dieser Reaktion des Körpers liegt wahrscheinlich sowohl der Einfluß der mechanischen Ventilation als auch die Abnahme des Sympatikotonus mit nachfolgender Dilatation der venösen Kapazitätsgefäße zugrunde (Hoeft und Buhre 1995). Dieser Abfall des intrathorakalen Volumens konnte nicht ausreichend durch Flüssigkeitssubstitution während der Anästhesieeinleitung kompensiert werden (s. Tabelle 8-2).

Die festgehaltenen und nach Auswertung der registrierten Dilutionskurven ermittelten Kreislaufparameter werden in der darauffolgenden Tabelle 8-2 dargestellt. 


\begin{tabular}{|c|c|c|c|c|c|c|c|c|c|c|c|}
\hline & & $\bar{x}$ & sd & sem & & & & $\bar{x}$ & sd & sem & \\
\hline$\underset{\left[\mathrm{min}^{-1}\right]}{\mathbf{H f}}$ & $\begin{array}{l}\text { v.N. } \\
\text { n.N. }\end{array}$ & $\begin{array}{l}65,8 \\
58,4\end{array}$ & $\begin{array}{l} \pm 10,5 \\
\pm 10,2\end{array}$ & $\begin{array}{l}2,7 \\
2,6\end{array}$ & $* *$ & $\begin{array}{c}\mathbf{V}_{\mathrm{d}-\text {-zirk }} \\
{\left[\mathrm{ml} \cdot \mathrm{kg}^{-1}\right]}\end{array}$ & $\begin{array}{l}\text { v.N. } \\
\text { n.N. }\end{array}$ & $\begin{array}{l}40,9 \\
44,0\end{array}$ & $\begin{array}{l} \pm \quad 3,7 \\
\pm \quad 3,9\end{array}$ & $\begin{array}{l}1,0 \\
1,0\end{array}$ & $* *$ \\
\hline $\begin{array}{c}\text { MAP } \\
{[\mathrm{mmHg}]}\end{array}$ & $\begin{array}{l}\text { v.N. } \\
\text { n.N. }\end{array}$ & $\begin{array}{l}93,3 \\
75,2\end{array}$ & $\begin{array}{l} \pm 11,5 \\
\pm 10,2\end{array}$ & $\begin{array}{l}3,0 \\
2,6\end{array}$ & $* *$ & $\begin{array}{l}\mathbf{V}_{\text {d-zent }} / \\
\mathbf{V}_{\text {d-zirk }}\end{array}$ & $\begin{array}{l}\text { v.N. } \\
\text { n.N. }\end{array}$ & $\begin{array}{l}0,25 \\
0,21\end{array}$ & $\begin{array}{l} \pm 0,03 \\
\pm 0,03\end{array}$ & $\begin{array}{l}0,01 \\
0,01\end{array}$ & $* *$ \\
\hline $\begin{array}{l}\text { MPAP } \\
{[\mathrm{mmHg}]}\end{array}$ & $\begin{array}{l}\text { v.N. } \\
\text { n.N. }\end{array}$ & $\begin{array}{l}19,2 \\
18,7\end{array}$ & $\begin{array}{l} \pm \quad 6,8 \\
\pm \quad 5,8\end{array}$ & $\begin{array}{l}1,8 \\
1,6\end{array}$ & & $\begin{array}{c}\mathbf{V}_{\mathrm{d}-\mathbf{1 k}} \\
{\left[\mathrm{ml} \cdot \mathrm{kg}^{-1}\right]}\end{array}$ & $\begin{array}{l}\text { v.N. } \\
\text { n.N. }\end{array}$ & $\begin{array}{l}15,9 \\
15,1\end{array}$ & $\begin{array}{l} \pm 2,6 \\
\pm \quad 2,9\end{array}$ & $\begin{array}{l}0,7 \\
0,8\end{array}$ & \\
\hline $\begin{array}{c}\text { ZVD } \\
{[\mathrm{mmHg}]}\end{array}$ & $\begin{array}{l}\text { v.N. } \\
\text { n.N. }\end{array}$ & $\begin{array}{c}7,0 \\
10,5\end{array}$ & $\begin{array}{l} \pm \quad 3,3 \\
\pm \quad 5,0 \\
\end{array}$ & $\begin{array}{l}0,9 \\
1,3 \\
\end{array}$ & $* *$ & $\begin{array}{c}\mathrm{Cl}_{1 \mathbf{k}} \\
{\left[1 \cdot \mathrm{min}^{-1} \cdot \mathrm{m}^{-2}\right]}\end{array}$ & $\begin{array}{l}\text { v.N. } \\
\text { n.N. }\end{array}$ & $\begin{array}{l}1,67 \\
1,30\end{array}$ & $\begin{array}{l} \pm 0,37 \\
\pm \quad 0,40\end{array}$ & $\begin{array}{l}0,09 \\
0,10\end{array}$ & $* *$ \\
\hline $\begin{array}{l}\text { PCWP } \\
{[\mathrm{mmHg}]}\end{array}$ & $\begin{array}{l}\text { v.N. } \\
\text { n.N. }\end{array}$ & $\begin{array}{l}11,8 \\
13,8\end{array}$ & $\begin{array}{l} \pm \quad 5,1 \\
\pm \quad 4,9\end{array}$ & $\begin{array}{l}1,4 \\
1,3\end{array}$ & $*$ & $\begin{array}{c}\mathbf{V}_{\mathrm{d}-2 \mathbf{k}} \\
{\left[\mathrm{ml} \cdot \mathrm{kg}^{-1}\right]}\end{array}$ & $\begin{array}{l}\text { v.N. } \\
\text { n.N. }\end{array}$ & $\begin{array}{l}25,0 \\
28,8\end{array}$ & $\begin{array}{l} \pm \quad 3,7 \\
\pm \quad 3,7\end{array}$ & $\begin{array}{l}0,96 \\
0,96\end{array}$ & $* *$ \\
\hline$\underset{\left[1 \cdot \mathrm{min}^{-1} \cdot \mathrm{m}^{-2}\right]}{\mathbf{C l}}$ & $\begin{array}{l}\text { v.N. } \\
\text { n.N. }\end{array}$ & $\begin{array}{l}2,6 \\
2,1 \\
\end{array}$ & $\begin{array}{ll} \pm & 0,6 \\
\pm & 0,4 \\
\end{array}$ & $\begin{array}{l}0,14 \\
0,11 \\
\end{array}$ & $* *$ & $\underset{\left[1 \cdot \mathrm{min}^{-1} \cdot \mathrm{m}^{-2}\right]}{\mathrm{Cl}_{2 k}}$ & $\begin{array}{l}\text { v.N. } \\
\text { n.N. }\end{array}$ & $\begin{array}{l}0,83 \\
0,84 \\
\end{array}$ & $\begin{array}{ll} \pm & 0,16 \\
\pm & 0,18 \\
\end{array}$ & $\begin{array}{l}0,04 \\
0,05 \\
\end{array}$ & \\
\hline $\begin{array}{c}\text { SVI } \\
{\left[\mathrm{ml} \cdot \mathrm{m}^{-2}\right]}\end{array}$ & $\begin{array}{l}\text { v.N. } \\
\text { n.N. }\end{array}$ & $\begin{array}{l}39,2 \\
37,0 \\
\end{array}$ & $\begin{array}{l} \pm \quad 6,9 \\
\pm \quad 5,7 \\
\end{array}$ & $\begin{array}{l}1,8 \\
1,5 \\
\end{array}$ & & $\begin{array}{c}\mathbf{V}_{\mathrm{d}-\mathrm{p}} \\
{\left[\mathrm{ml} \cdot \mathrm{kg}^{-1}\right]}\end{array}$ & $\begin{array}{l}\text { v.N. } \\
\text { n.N. }\end{array}$ & $\begin{array}{l}28,7 \\
32,6 \\
\end{array}$ & $\begin{array}{l} \pm \quad 4,0 \\
\pm \quad 4,1 \\
\end{array}$ & $\begin{array}{l}1,2 \\
1,1 \\
\end{array}$ & $* *$ \\
\hline $\begin{array}{c}\text { PVRI } \\
{[\text { dyn } \cdot \mathrm{sec} \text {. }} \\
\left.\mathrm{m}^{-2} \cdot \mathrm{cm}^{-5}\right]\end{array}$ & $\begin{array}{l}\text { v.N. } \\
\text { n.N. }\end{array}$ & $\begin{array}{l}239,8 \\
189,1\end{array}$ & $\begin{array}{lc} \pm & 129 \\
\pm & 76,1\end{array}$ & $\begin{array}{l}35,8 \\
20,3\end{array}$ & & $\begin{array}{c}V_{\mathrm{d}-1 \mathrm{k}(\mathrm{p})} \\
{\left[\mathrm{ml} \cdot \mathrm{kg}^{-1}\right]}\end{array}$ & $\begin{array}{l}\text { v.N. } \\
\text { n.N. }\end{array}$ & $\begin{array}{l}8,09 \\
8,22\end{array}$ & $\begin{array}{l} \pm 2,23 \\
\pm 1,93\end{array}$ & $\begin{array}{l}0,64 \\
0,54\end{array}$ & \\
\hline $\begin{array}{c}\text { SVRI } \\
{[\mathrm{dyn} \cdot \mathrm{sec}} \\
\left.\mathrm{m}^{-2} \cdot \mathrm{cm}^{-5}\right]\end{array}$ & $\begin{array}{l}\text { v.N. } \\
\text { n.N. }\end{array}$ & $\begin{array}{l}2692 \\
2459\end{array}$ & $\begin{array}{l} \pm \quad 487 \\
\pm \quad 637\end{array}$ & $\begin{array}{l}126 \\
164\end{array}$ & & $\begin{array}{c}V_{\mathrm{d}-2 \mathrm{k}(\mathrm{p})} \\
{\left[\mathrm{ml} \cdot \mathrm{kg}^{-1}\right]}\end{array}$ & $\begin{array}{l}\text { v.N. } \\
\text { n.N. }\end{array}$ & $\begin{array}{l}20,6 \\
24,4\end{array}$ & $\begin{array}{l} \pm \quad 3,3 \\
\pm \quad 3,2\end{array}$ & $\begin{array}{l}1,0 \\
0,9\end{array}$ & $* *$ \\
\hline $\begin{array}{c}V_{\text {d-zent }} \\
{\left[\mathrm{ml} \cdot \mathrm{kg}^{-1}\right]}\end{array}$ & $\begin{array}{l}\text { v.N. } \\
\text { n.N. }\end{array}$ & $\begin{array}{c}10,2 \\
9,4 \\
\end{array}$ & $\begin{array}{l} \pm 1,5 \\
\pm \quad 1,0 \\
\end{array}$ & $\begin{array}{l}0,4 \\
0,3 \\
\end{array}$ & & $\begin{array}{c}\text { Kristalloide } \\
{\left[\mathrm{ml} \cdot \mathrm{kg}^{-1}\right]}\end{array}$ & $\begin{array}{r}\text { bis } \\
\text { n.N. }\end{array}$ & 21,52 & $\pm 6,35$ & 1,64 & \\
\hline $\begin{array}{c}\mathbf{V}_{\mathrm{d}-\mathrm{ITBV}} \\
{\left[\mathrm{ml} \cdot \mathrm{kg}^{-1}\right]}\end{array}$ & $\begin{array}{l}\text { v.N. } \\
\text { n.N. }\end{array}$ & $\begin{array}{l}17,7 \\
16,4\end{array}$ & $\begin{array}{l} \pm 1,5 \\
\pm \quad 1,3\end{array}$ & $\begin{array}{l}0,4 \\
0,3\end{array}$ & $* *$ & $\begin{array}{l}\text { Kolloide } \\
{\left[\mathrm{ml} \cdot \mathrm{kg}^{-1}\right]}\end{array}$ & $\begin{array}{l}\text { bis } \\
\text { n.N. }\end{array}$ & 7,91 & $\pm 5,69$ & 1,47 & \\
\hline $\begin{array}{l}\text { EVLW } \\
{\left[\mathrm{ml} \cdot \mathrm{kg}^{-1}\right]}\end{array}$ & $\begin{array}{l}\text { v.N. } \\
\text { n.N. }\end{array}$ & $\begin{array}{l}5,11 \\
5,31\end{array}$ & $\begin{array}{ll} \pm & 0,98 \\
\pm & 0,79\end{array}$ & $\begin{array}{l}0,31 \\
0,24\end{array}$ & & $\begin{array}{c}\text { Gesamtbilanz } \\
{\left[\mathrm{ml}^{\left.-\mathrm{kg}^{-1}\right]}\right.}\end{array}$ & $\begin{array}{l}\text { bis } \\
\text { n.N. }\end{array}$ & 29,23 & $\pm 9,73$ & 2,51 & \\
\hline
\end{tabular}

Tabelle 8-2. Einfluß der Narkoseeinleitung mit Sufentanil auf die hämodynamischen Parameter und die Flüssigkeitsverteilung

v.N. = vor Narkoseeinleitung; n.N. = nach Narkoseeinleitung

$H f=$ Herzfrequenz; $M A P=$ arterieller Mitteldruck; $M P A P=$ pulmonalarterieller Mitteldruck;

$Z V D=$ zentraler Venendruck; $P C W P=$ pulmonalkapillärer Verschlußdruck;

$C I=$ Herzzeitvolumenindex; $S V I=$ Schlagvolumenindex;

$P V R I=$ pulmonaler Gefäßwiderstandsindex; $S V R I=$ Peripherer Gefäßwiderstandsindex;

$V_{d-z e n t}=$,Zentrales“ Blutvolumen; $V_{d-I T B V}=$,Intrathorakales“ Blutvolumen;

$E V L W=$,Extravakuläres Lungenwasser“; $V_{d-\text { zirk }}=$,Zirkulierendes,, Blutvolumen;

$V_{d-z e n t} / V_{d-z i r k}=$ Anteil des ,zentralen" am ,zirkulierenden" Blutvolumen;

$V_{d-l k, 2 k}=$ Volumen der Einzelkompartimente im Gesamtkreislauf;

$C I_{l k, 2 k}=$ Fluß zu den Einzelkompartimenten; $V_{d-p}=$,Peripheres zirkulierendes“ Blutvolumen;

$V_{d-l k(p), 2 k(p)}=$ Volumen der Einzelkompartimente im großen Kreislauf

$*(* *): \mathrm{p}<0,05(0,01):$ v.N. vs. n.N. (Student's t-test für verbundene Stichproben) 


\section{Literaturverzeichnis}

Altman und Bland 1983

Altman DG, Bland JM (1983): Measurement in medicine: the analysis of method comparison studies. Statistician 32, 307-317

Anderson 1989

Anderson DJ (1989): Determination of the lower limit of detection [letter; comment].

Clin Chem 35, 2152-2153

Arndt 1986

Arndt JO (1986): he low pressure system: the integrated function of veins.

Eur J Anaesthesiol $\underline{3}$, 343-370

Baker 1966

Baker KJ (1966): Binding of sulfobromophthalein (BSP) sodium and indocyanine green (ICG) by plasma alpha-1 lipoproteins.

Proc Soc Exp Biol Med 122, 957-963

Bassingthwaighte 1967

Bassingthwaighte JB (1967): Circulatory transport and the convolution integral.

Mayo Clin Proc 42, 137-154

Bassingthwaighte und Ackerman 1967

Bassingthwaighte JB, Ackerman FH (1967):

Mathematical linearity of circulatory transport. J Appl Physiol 22, 879-888

Bassingthwaighte et al. 1966a Bassingthwaighte JB, Ackerman FH, Wood EH (1966): Applications of the lagged normal density curve as a model for arterial dilution curves. Circ Res 18 , 398-415

Bassingthwaighte et al. 1966b Bassingthwaighte JB, Warner HR, Wood EH (1966): Analog computer analysis of dispersion of indicator in the circulation.

Med Res Eng $\underline{5}, 30-37$

Benya et al. 1989

Benya R, Quintana J, Brundage B (1989): Adverse reactions to indocyanine green: a case report and a review of the literature.

Cathet Cardiovasc Diagn 17, 231-233

Björnsson et al. 1983

Björnsson OG, Murphy R, Chadwick VS, Björnsson S (1983): Physicochemical studies on indocyanine green: molar lineic Absorbance, pH tolerance, activation energy and rate of decay in various solvents. J Clin Chem Clin Biochem 21, 453-458

Blomqvist et al. 1990

Blomqvist $\mathrm{H}$, Berg B, Frostell C, Wickerts CJ, Hedenstierna G (1990): Net fluid leakage (LN) in experimental pulmonary oedema in the dog. Acta Anaesthesiol Scand $\underline{34}$, 377-383

Böck 1987

Böck J: Die Farbstoff-Kälteverdünnungstechnik zur Bestimmung des extravaskulären Lungenwassers. Med. Dissertation Göttingen 1987
Böck et al. 1988

Böck JC, Deuflhard P, Hoeft A, Korb H, Steinmann J, Wolpers HG, Hellige G (1988): Evaluation of monoexponential extrapolation of transpulmonary thermal-dye kinetics by use of a new model-free deconvolution algorithm.

Med Instrum 22, 20-28

Boldt et al. 1986

Boldt J, v. Bormann B, Kling D, Jooss D, Moosdorf R, Hempelmann G (1986): Der Einfluß von

Glucocorticoiden auf das extravasculäre Lungenwasser nach extracorporaler Zirkulation. Anaesthesist 35, 238-244

Boldt et al. 1988

Boldt J, von Bormann B, Kling D, Scheld $H$, Hempelmann G (1988): Influence of acute normovolemic hemodilution on extravascular lung water in cardiac surgery.

Crit Care Med 16, 336-339

Boldt et al. 1995

Boldt J, Osmer C, Schindler E, Linke LC, Stertmann WA, Hempelmann G (1995): Circulating adhesion molecules in cardiac operations: Influence of highdose aprotinin.

Ann Thorac Surg 59, 100-105

Bradley und Barr 1968

Bradley EC, Barr JW (1968): Determination of blood volume using indocyanine green (cardio-green) dye. Life Sci $\underline{7}, 1001-1007$

Butler et al. 1992

Butler J, Chong GL, Baigrie RJ, Pillai R, Westaby S, Rocker GM (1992): Cytokine responses to cardiopulmonary bypass with membrane and bubble oxygenation. Ann Thorac Surg 포, 833-838

Butler et al. 1993

Butler J, Rocker GM, Westaby S (1993): Inflammatory response to cardiopulmonary bypass. Ann Thorac Surg 55, 552-559

Caldini et al. 1974 Caldini P, Permutt S, Waddell JA, Riley RL (1974): Effect of epinephrine on pressure, flow, and volume relationships in the systemic circulation of dogs. Circ Res 34, 606-623

Chenoweth et al. 1981

Chenoweth DE, Cooper SW, Hugli TE, Stewart RW, Blackstone EH, Kirklin JW (1981): Complement activation during cardiopulmonary bypass: evidence for generation of $\mathrm{C} 3 \mathrm{a}$ and $\mathrm{C} 5 \mathrm{a}$ anaphylatoxins. N Engl J Med 304, 497-503

Chiou 1979

Chiou WL (1979): Potential pitfalls in the conventional pharmacokinetic studies: effects of the initial mixing of drug in blood and the pulmonary first-pass elimination J Pharmacokinet Biopharm ㄱ, 527-536

Comper 1984

Comper WD: Interstitium;

in: Edema; hrsg. v. Staub NC, Taylor AE;

Raven Press, New York 1984 
Cutler 1979

Cutler DJ (1979): A linear recirculation model for drug disposition.

J Pharmacokinet Biopharm ㄱ, 101-116

Dauber et al. 1993

Dauber I, Parsons P, Welsh C, Giclas P, Whitman G, Wheeler G, Horwitz L, Weil J (1993): Peripheral bypass-induced pulmonary and coronary vascular Injury. Association with increased levels of tumor necrosis factor.

Circulation 88, 726-735

Deng et al. 1995

Deng MC, Wiedner M, Erren M, Möllhoff T, Assmann G, Scheld HH (1995): Arterial and venous cytokine response to cardiopulmonary bypass for low risk $\mathrm{CABG}$ and relation to hemodynamics. Eur J Cardio-thorac Surg $\underline{9}$, 22-29

Deuflhard und Apostolescu 1980

Deuflhard P, Apostolescu V: A study of the GaussNewton method for the solution of nonlinear least squares problems; in: Special topics of applied mathematics; hrsg. v. Frehse J, Pallaschke D, Trottenberg U; North Holland publishing company, Amsterdam 1980, 129-150

Dodrill 1958

Dodrill FD: The effects of total body perfusion upon the lungs; in: Extracorporeal circulation; hrsg. v. Garrott JA; Thomas, Springfield 1958, 327-335

Elliott und Finn 1993

Elliott MJ, Finn AHR (1993): Interaction between neutrophils and endothelium.

Ann Thorac Surg 트, 1503-1508

Finn et al. 1993

Finn A, Moat N, Rebuck N, Klein N, Strobel S, Elliott M (1993): Changes in neutrophil CD11b/CD18 and Lselectin expression and release of interleukin 8 and elastase in paediatric cardiopulmonary bypass. Agents Actions 38 , Special Conference Issue

Fox und Wood 1960

Fox IJ, Wood EH (1960): Indocyanine green: Physical and physiological properties.

Mayo Clin Proc $\underline{35}$, 732-744

Fox et al. 1957

Fox IJ, Brooker LGS, Heseltine DW, Essex HE, Wood EH (1957): A tricarbocyanine dye for continuous recording of dilution curves in whole blood independent of variations in blood oxygen saturation. Proc Staff Meet Mayo Clin 32, 478-484

Frostell et al. 1990

Frostell C, Blomqvist $\mathrm{H}$, Wickerts CJ, Hedenstierna G (1990): Lung fluid balance evaluated by the rate of change of extravascular lung water content. Acta Anaesthesiol Scand 34, 362-369

Gaebler 1943

Gaebler $\mathrm{OH}$ (1943): The relationship of extinction to wave-length in turbid sera an other suspensions. J Biol Chem 149, 251

Gallagher et al. 1985

Gallagher JD, Moore RA, Kerns D, Jose AB, Botros

SB, Clark DL (1985): Effects of advanced age on extravascular lung water accumulation during coronary artery bypass surgery.

Crit Care Med $\underline{13}, 68-71$
Gauer 1972

Gauer $\mathrm{OH}$ : Volumenregulation;

in: Physiologie des Menschen, Band 3: Herz und Kreislauf (Trautwein W, Gauer OH, Koepchen HP); hrsg. v. Gauer OH, Kramer K, Jung R;

Urban \& Schwarzenberg, München 1972, 306-326

Gebhard 1990

Gebhard MM (1990): Myocardial protection and ischemia tolerance of the globally ischemic heart. Thorac Cardiovasc Surg 38, 55-59

Giannaris et al. 1997

Giannaris S, Schorn B, Wietasch G, Scholz M, Buhre W, Weyland A, Hoeft A (1997): Effect of dexamethasone on oedema formation in patients undergoing coronary bypass surgery.

$\mathrm{Br} J$ Anaesth 78 (Suppl. 2), 12

Gibson und Evans 1937

Gibson JG, Evans WA (1937): Clinical studies of the blood. I. Clinical application of a method employing the azo dye "Evans blue" and the spectralphotometer. J Clin Invest 16, 301

Glauser und Fairman 1985

Glauser FL, Fairman RP (1985): The uncertain role of the neutrophil in increased permeability pulmonary edema.

Chest $88,601-607$

Grainger et al. 1983

Grainger SL, Keeling PW, Brown IM, Marigold JH, Thompson RP (1983): Clearance and non-invasive determination of the hepatic extraction of indocyanine green in baboons and man. Clin Sci $\underline{64}, 207-212$

Gregersen et al. 1935

Gregersen MI, Gibson JG, Stead EA (1935): Plasma volume determination with dyes; errors in colorimetry; use of the blue dye T-1824.

Am J Physiol 133, 54

Green 1979

Green JF: Determinations of systemic blood flow; in: Cardiovascular Physiology III, vol. 18; hrsg. v. Guyton AC, Young DB;

University Park Press, Baltimore 1979, 33-65

Grodins 1962

Grodins FS (1962): Basic concepts in the determination of vascular volume by indicator-dilution methods.

Circ Res 10, 429-446

Gu et al. 1993

Gu YJ, van Oeveren W, Akkerman C, Boonstra PW, Huyzen RJ, Wildevuur CRH (1993): Heparin-coated circuits reduce the inflammatory response to cardiopulmonary bypass. Ann Thorac Surg 55: 917-922

Hachenberg et al. 1993

Hachenberg T, Tenling A, Rothen HU, Nystrom SO, Tyden H, Hedenstierna G (1993): Thoracic intravascular and extravascular fluid volumes in cardiac surgical patients.

Anesthesiology $\underline{79}$, 976-984

Haeffner-Cavaillon et al. 1989

Haeffner-Cavaillon N, Roussellier N, Ponzio O, Carreno MP, Laude M, Carpentier A, Kazatchkine MD (1989): Induction of interleukin-1 production in patients undergoing cardiopulmonary bypass. J Thorac Cardiovasc Surg 모, 1100-1106 
Haller et al. 1992

Haller M, Brechtelsbauer $\mathrm{H}$, Finsterer U, Forst $\mathrm{H}$, Bein Th, Briegel J, Peter K (1992): Bestimmung des Plasmavolumens mit Indocyaningrün beim Menschen. Anaesthesist 41, 115-120

Hamilton et al. 1928

Hamilton WF, Moore JW, Kinsman JM, Spurling RG (1928): Simultaneous determination of the pulmonary and systemic circulation times in man and of a figure related to the cardiac output.

Am J Physiol 84, 338-344

Haneda und Horiuchi 1986

Haneda K, Horiuchi T (1986): A method for measurement of total circulating blood volume using indocyanine green.

Tohoku J Exp Med 148, 49-56

Henthorn et al. 1992

Henthorn TK, Avram MJ, Krejcie TC, Shanks CA, Asada A, Kaczynski DA (1992): Minimal compartmental model of circulatory mixing of indocyanine green.

Am J Physiol 262, H903-H910

Hoeft 1995

Hoeft A: Transpulmonary indicator dilution: An alternative approach for hemodynamic monitoring; in: Yearbook of Intensive Care and Emergency Medicine; Springer, Berlin/Heidelberg/New York 1995, 593-605

Hoeft und Buhre 1995

Hoeft A, Buhre W: Anaesthesia and the cardiovascular system; in: Cardiovascular Physiology; hrsg. v. Priebe H-J, Skarvan K;

BMJ Publishing Group, London 1995, 272-306

Hoeft et al. 1991

Hoeft A, Korb H, Mehlhorn U, Stephan H, Sonntag H (1991): Priming of cardiopulmonary bypass with human albumin or Ringer lactate: effect on colloid osmotic pressure and extravascular lung water. $\mathrm{Br} \mathrm{J}$ Anaesth 66, 73-80

Hoeft et al. 1994

Hoeft A, Schorn B, Weyland A, Scholz M, Buhre W, Stepanek E, Allen SJ, Sonntag H (1994): Bedside assessment of intravascular volume status in patients undergoing coronary bypass surgery. Anesthesiology $\underline{81}, 76-86$

Hurley 1975

Hurley PJ (1975): Red cell and plasma volumes in normal adults.

J Nucl Med 16, 46-52

Jacobson 1968

Jacobson ED (1968): A physiologic approach to shock. $\mathrm{N}$ Engl J Med 278, 834-839

Jansen et al. 1991a

Jansen NJG, van Oeveren W, v. d. Broek L,

Oudemans-van Straaten HM, Stoutenbeek CP, Chang Njoek Joen M, Roozendaal KJ, Eysman L, Wildevuur $\mathrm{CRH}$ (1991): Inhibition by dexamethasone of the reperfusion phenomena in cardiopulmonary bypass. J Thorac Cardiovasc Surg 102, 515-525

Jansen et al. 1991b

Jansen NJG, van Oeveren W, van Vliet $\mathrm{MH}$, Stoutenbeek CP, Eysman L, Wildevuur CRH (1991): The role of different types of corticosteroids on the inflammatory mediators in cardiopulmonary bypass. Eur J Cardio-thorac Surg $\underline{5}, 211-217$
Jansen et al. 1992

Jansen NJG, van Oeveren W, Gu YJ, van Vliet MH, Eijsman L, Wildevuur CRH (1992): Endotoxin release and tumor necrosis factor formation during cardiopulmonary bypass.

Ann Thorac Surg 54, 744-748

Kalfin et al. 1993

Kalfin RE, Engelman RM, Rousou JA, Flack III JE, Deaton DW, Kreutzer DL, Das DK (1993): Induction of Interleukin-8 Expression during cardiopulmonary bypass.

Circulation 88 [part 2], 401-406

Keith et al. 1915

Keith NM, Rowntree LG, Geraghty JT (1915): A method for the determination of plasma and blood volume.

Arch Int Med 16, 547

Kirklin et al. 1983

Kirklin JK, Westaby S, Blackstone EH, Kirklin JW, Chenoweth DE, Pacifico AD (1983): Complement and the damaging effects of cardiopulmonary bypass. J Thorac Cardiovasc Surg $\underline{86}$, 845-857

Knopp und Bassingthwaighte 1969 Knopp TJ, Bassingthwaighte JB (1969): Effect of flow on transpulmonary circulatory transport functions. J Appl Physiol 27, 36-43

Knopp et al. 1976

Knopp TJ, Dobbs WA, Greenleaf JF,

Bassingthwaighte JB (1976): Transcoronary

intravascular transport functions obtained via a stable deconvolution technique.

Ann Biomed Eng 4, 44-59

Kreuzfelder et al. 1988

Kreuzfelder E, Joka T, Keinecke HO, Obertacke U, Schmit-Neuerburg KP, Nakhosteen JA, Paar D, Scheiermann N (1988): Adult respiratory distress syndrome as a specific manifestation of a general permeability defect in trauma patients.

Am Rev Respir Dis 137, 95-99

Kuntz und May 1983

Kuntz HD, May B (1983): Quantitative Leberfunktionsdiagnostik.

Münch Med Wochenschr 125, 678

Landsman et al. 1976

Landsman ML, Kwant G, Mook GA, Zijlstra WG (1976): Light absorbing properties, stability and spectral stabilization of indocyanine green. J Appl Physiol 40, 575-583

Lassen und Perl 1979

Lassen NA, Perl W: Tracer kinetic methods in medical physiology;

Raven Press, New York 1979

Lewis et al. 1979

Lewis FR, Elings VB, Sturm JA (1979): Bedside

measurement of lung water.

J Surg Res 27, 250-261

Lewis et al. 1982

Lewis F, Elings V, Hill S (1982): The measurement of extravascular lung water by thermal-green dye dilution. Ann N Y Acad Sci 384, 394-410

Lichtwarck-Aschoff et al. 1992

Lichtwarck-Aschoff M, Zeravik J, Pfeiffer UJ (1992): Intrathoracic blood volume accurately reflects circulatory volume status in critically ill patients with mechanical ventilation.

Int Care Med 18, 142-147 
Lumb 1987

Lumb PD (1987): A comparison between $25 \%$ albumin and $6 \%$ hydroxyethyl starch solutions on lung water accumulation during and immediately after cardiopulmonary bypass.

Ann Surg 206, 210-213

Mehlhorn et al. 1990

Mehlhorn U, Hoeft A, Borowski A, Korb H, Stephan H, de Vivie R (1990): Messung des extravaskulären Lungenwassers am Patienten: Höhere Genauigkeit bei pulmonalarteriellem und aortalem Indikatornachweis. ECC International 2, 91-104

Meier und Zierler 1954

Meier P, Zierler KL (1954): On the theory of the indicator-dilution method for measurement of blood flow and volume.

J Appl Physiol $\underline{6}, 731-744$

Menasché et al. 1994

Menasché P, Haydar S, Peynet J, Du Buit C, Merval R, Bloch G, Piwnica A, Tedgui A (1994): A potential mechanism of vasodilation after warm heart surgery: the temperature-dependent release of cytokines. J Thorac Cardiovasc Surg 107, 293-299

Mickulecky 1973

Mickulecky M (1973): The compartmental analysis of the indocyanine green plasma clearance in normal human subjects with the aid of a digital computer. Scripta Medica 46, 469-476

Mihm et al. 1987

Mihm FG, Feeley TW, Jamieson SW (1987): Thermal dye double indicator dilution measurement of lung water in man: comparison with gravimetric measurements.

Thorax $\underline{42}, 72-76$

Miranda et al. 1982 Miranda DR, Stoutenbeek C, Karliczek G, Rating W (1982): Effects of dexamethasone on the early postoperative course after coronary artery bypass surgery.

Thorac Cardiovasc Surg 으, 21-7

Moens et al. 1962

Moens RS, Busset R, Collet RA, Mach RS (1962):

Utilisation de l'albumine- ${ }^{131}$ (RIHSA) pour la détermination du volume plasmatique et du volume sanguin chez le sujet normal.

Schweiz Med Wochenschr 92, 1660-1667; 1697-1703

Möllmann et al. 1984

Möllmann HW, Barth J, Schmidt W, Rohdewald P (1984): Schocklunge (ARDS): Glucocorticoide? Anästh Intensiv Notfallmed 19, 99

Moos und Craigo 1994

Moos J, Craigo PA: The autonomic nervous system; in: Anesthesia, vol. I, 4th edition; hrsg. v. Miller RD; Churchill Livingstone, New York 1994, 523-575

Nakai 1981

Nakai M (1981): Computation of transport function using multiple regression analysis. Am J Physiol 240, H133-44

Nielsen und Nielsen 1962

Nielsen MH, Nielsen NC (1962): Spectrophotometric determination of evans blue dye in plasma with individual correction for blank density by a modified gaeblers method.

Scand J Clin Lab Invest 14, 605-617
Nielsen $1963 a$

Nielsen NC (1963): Correction for blank density in spectrophotometric dye determination in turbid plasma within the spectral range 600 to 920 nanometers. Scand J Clin Lab Invest 15, 610-612

Nielsen 1963b

Nielsen NC (1963): Spectrophotometric determination of indocyanine green in plasma especially with a view to an improved correction for blank density. Scand J Clin Lab Invest 15, 613-621

Paumgartner 1975

Paumgartner $\mathrm{G}$ (1975): The handling of indocyanine grreen by the liver.

Schweiz Med Wochenschr 105, 1

Pearce und Beazell 1966

Pearce ML, Beazell JW (1966): The measurement of pulmonary parenchymal volume by thermal indicator dilution.

Clin Res $\underline{14}, 182$

Pfeiffer 1990

Pfeiffer UJ: Das intrathorakale Blutvolumen als hämodynamischer Leitparameter; in: Anaesthesiologie und Intensivmedizin, vol. 214; hrsg. v. Bergmann H, Brückner JB, Gemperle M, Henschel WF, Mayrhofer O, Meßmer K, Peter K; Springer, Berlin 1990

Pfeiffer et. al. 1986

Pfeiffer UJ, Aschenbrenner G, Kolb E, Blümel G, Pfeiffer H-G (1986): Die Messung des intrathorakalen Blutvolumens als sensitiver Parameter zur Steuerung der Volumensubstitution.

Langenbecks Arch Chir Forum 으, 203-207

Piepenbrock et al. 1977

Piepenbrock S, Hempelmann G, Westermann C (1977): Massive doses of methylprednisolone $(30 \mathrm{mg} / \mathrm{kg})$ in man: Immediate hemodynamic effects in "low output state". Int Care Med 3, 69

Ratcliff et al. 1973

Ratcliff NB, Young WG, Hackel DB, Mikat E, Wilson JW (1973): Pulmonary injury secondary to extracorporeal circulation. J Thorac Cardiovasc Surg $\underline{65}, 425$

Reeve und Veall 1949

Reeve EB, Veall H (1949): A simplified method for the determination of circulating redcell volume with radioactive phosphorus. J Physiol Lond 108, 12

Schad et al. 1987

Schad H, Haider M, Brechtelsbauer H (1987):

Bestimmung des Plasmavolumens mit Indocyaningrun. Anaesthesist $\underline{36}, 608-614$

Schmidt und Thews 1990 Schmidt RF, Thews G (hrsg.): Physiologie des Menschen, 24. Auflage; Springer, Berlin 1990

Schorn 1995

Schorn B: Untersuchungen zum perioperativen intraund extravaskulären Volumenstatus herzchirurgischer Patienten.

Med. Habil.-Schr. Göttingen 1995 
Schorn et al. 1992

Schorn B, Hoeft A, Weyland A, Buhre W, Stepanek E, Scholz M, Dalichau H (1992): Perioperatives Verhalten des extravaskulären Lungenwassers nach Mitralklappenersatz.

Z Herz Thorax Gefäßchir $\underline{6}$, 251-256

Schüpbach et al. 1978

Schüpbach P, Pappova E, Schilt W, Kollar J, Koller M, Sipos P, Vucic D (1978): Perfusate oncotic pressure during cardiopulmonary bypass. Optimum level as determined by metabolic acidosis, tissue edema, and renal function.

Vox Sang 35, 332-344

Schwinn et al. 1994

Schwinn DA, Watkins WD, Leslie JB: Basic principles of pharmacology related to anesthesia;

in: Anesthesia, vol. I, 4th edition; hrsg. v. Miller RD; Churchill Livingstone, New York 1994, 43-65

Sellevold und Jynge 1985

Sellevold OFM, Jynge P (1985): Steroids and cardioplegia. An experimental evaluation of glucocorticoid supplementation to cardioplegic solutions in clinical use.

Thorac Cardiovasc Surg $\underline{33}, 65$

Shinohara et al. 1996

Shinohara H, Tanaka A, Kitai T, Yanabu N, Inomoto T, Satoh S, Hatano E, Yamaoka Y, Hirao K (1996): Direct measurement of hepatic indocyanine green clearance with near-infrared spectroscopy: separate evaluation of uptake and removal.

Hepatology 23(1), 137-144

Shoemaker et al. 1988

Shoemaker WC, Appel PL, Kram HB, Nathan RC Thompson JL (1988): Multicomponent noninvasive physiologic monitoring of circulatory function. Crit Care Med 16, 482-490

Sibbald et al. 1981

Sibbald WJ, Anderson RR, Reid B, Holliday RL, Driedger AA (1981): Alveo-capillary permeability in human septic ARDS

Chest 79, 133

Speich et al. 1988

Speich R, Saesseli B, Hoffmann U, Neftel KA, Reichen $J$ (1988): Anaphylactoid reactions after indocyanine green administration.

Ann Intern Med 109: 345-346

Staub 1974

Staub NC (1974): „State of the art“ review.

Pathogenesis of pulmonary edema.

Am Rev Respir Dis 109, 358-372

Stephenson 1948

Stephenson JL (1948): Theory of the measurement of blood flow by dilution of an indicator.

Bull Math Biophys 10, 117-121

Stoeckel et al. 1980

Stoeckel K, McNamara PJ, McLean AJ, duSouich P, Lalka D, Gibaldi M (1980): Nonlinear pharmacokinetics of indocyanine green in the rabbit and the rat. J Pharmacokinet Biopharm 8, 483-496

Swan und Nelson 1971

Swan H, Nelson AW (1971): Blood volume I: critique: spun vs. isotope hematocrit; ${ }^{125} \mathrm{RIHSA}$ vs. ${ }^{51} \mathrm{CrRBC}$. Ann Surg 173, 481-495
Tikhonov und Arsenin 1977

Tikhonov AM, Arsenin VY: Solutions of ill-posed problems.

Winston \& Sons, New York 1977

van Rossum et al. 1989

van Rossum JM, de Bie JE, van Lingen G, Teeuwen HW (1989): Pharmacokinetics from a dynamical systems point of view.

J Pharmacokinet Biopharm 17, 365-92

Wessel et al. 1971

Wessel HU, Paul MH, James GW, Grahn AR (1971): Limitations of thermal dilution curves for cardiac output determinations.

J Appl Physiol 30, 643-652

Westaby 1987

Westaby S (1987): Organ dysfunction after cardiopulmonary bypass. A systemic inflammatory reaction initiated by the extracorporeal circuit. Int Care Med 13, 89-95

Weyland 1984

Weyland A: Der Einfluß von Dexamethason auf den Gasaustausch nach extracorporaler Circulation. Med. Diss. Göttingen 1984

Weyland et al. 1995

Weyland A, Wietasch G, Hoeft A, Buhre W, Allgeier B, Weyland W, Kettler D (1995): Einfluß eines intrakardialen Links-rechts-Shunts auf pulmonalarterielle Thermodilutionsmessungen des Herzzeitvolumens. Untersuchungen an einem extrakorporalen Kreislaufmodell.

Anaesthesist 44, 13-23

Wiener-Kronish und Broaddus 1993

Wiener-Kronish JP, Broaddus VC (1993): Interrelationship of pleural and pulmonary interstitial liquid.

Annu Rev Physiol 55, 209-226

Wietasch 1995

Wietasch JKG: Die Doppelindikatordilution zur Quantifizierung von Herzzeit-volumen und LinksRechts-Shunt bei Patienten mit kongenitalem Vitium cordis. - Ein neuer systemanalytischer Ansatz Med. Diss. Göttingen 1995

Wilson 1972

Wilson JW (1972): Treatment or prevention of pulmonary cellular damage with pharmacologic doses of corticosteroids.

Surg Gynecol Obstet 134, 675

Zierler 1962a

Zierler KL: Circulation times and the theory of indicator-dilution methods for determining blood and volume; in: Handbook of physiology, sect 2/l: Circulation; hrsg. v. Society American Physiological; Washington DC/USA 1962, 585-615

Zierler 1962b

Zierler KL (1962): Theoretical basis of indicator-dilution methods for measuring flow and volume.

Circ Res 10, 393-407 


\section{Danksagung}

Mein besonderer Dank gilt meinen Lehrern Herrn Privatdozent Dr. med. Bernd Schorn und Herrn Prof. Dr. med. Andreas Hoeft für die Überlassung des Themas, die wissenschaftliche Anleitung und Betreuung und die ständige bereitwillige Förderung der Arbeit.

Meinen Freunden Dr. med. Götz Wietasch, Herrn Martin Scholz und Herrn Henning Cuhls danke ich für die sehr produktive Zusammenarbeit im Rahmen unserer Arbeitsgruppe in Göttingen und die freundliche und hilfreiche Durchsicht der Ergebnisse.

Ebenso danke ich Herrn Dr. med. Tilman von Spiegel, Herrn Privatdozent Dr. med. Michael Mohr und Herrn Privatdozent Dr. med. Andreas Weyland für die konstruktive Unterstützung und Hilfsbereitschaft bei der Narkoseeinleitung der Patienten.

Meinen Bruder Markos Giannaris und Frau Dr. med. Maria Tountopoulou danke ich vom Herzen dafür, daß sie mit ihrer Unterstützung und ihren weiterführenden Anregungen die Verwirklichung dieser Dissertation erst ermöglichten und mir mit ihrer Hilfe immer zur Seite standen.

Mein herzlicher Dank gilt schließlich Frau Dr. med. Christine Wüstenberg und Herrn Dr. med. Peter Dern nicht nur dafür, daß sie immer bereit waren, Korrektur zu lesen und mir Anregungen zur verständlichen Formulierung dieser Dissertationsschrift zu geben. Sondern vielmehr, daß sie mich durch viele Höhen und Tiefen begleitet haben. 